\title{
Characterization of cardiac progenitor cell activity in engineered heart muscle
}

\author{
Doctoral Thesis \\ In partial fulfillment of the requirements for the degree \\ "Doctor rerum naturalium (Dr. rer. nat.)" \\ in the Molecular Medicine Study Program \\ at the Georg-August University Göttingen
}

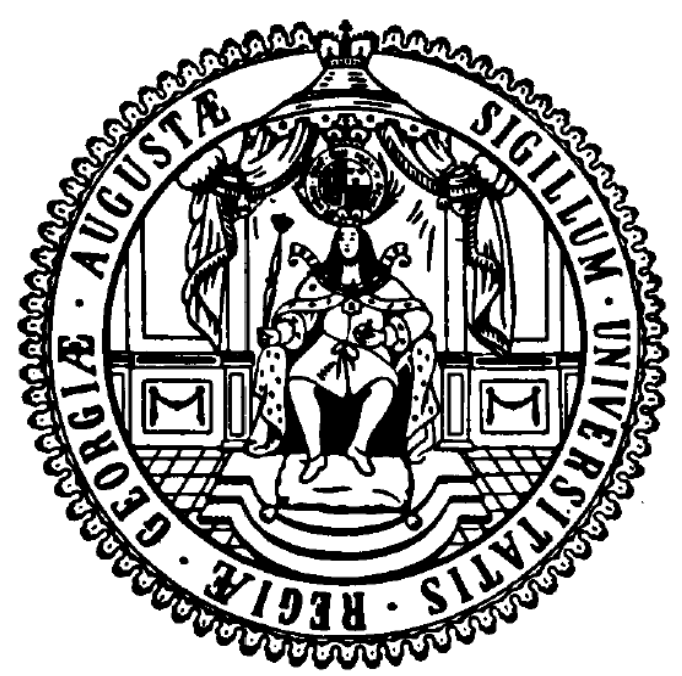

Submitted by

Elif Levent

born in Karabük, Turkey

Göttingen 2016 


\section{Members of the Thesis Committee:}

Prof. Dr. med. Wolfram-Hubertus Zimmermann (Supervisor)

Email: w.zimmermann@med.uni-goettingen.de

Phone: $\quad$ +49 (0) 551395781

Postal Address: Institute of Pharmacology and Toxicology University Medical Center Göttingen Georg-August University Göttingen Robert-Koch-Str. 40 37075 Göttingen Germany

Prof. Dr. Dörthe Katschinski (Second member of thesis committee)

Email: doerthe.katschinski@med.uni-goettingen.de

Phone: $\quad$ +49 (0) 551395896

Postal Address: Institute of Cardiovascular Physiology Humboldtallee 23 37073 Göttingen Germany

Prof. Dr. Viacheslav O. Nikolaev (Third member of thesis committee)

Email: $\quad$ v.nikolaev@uke.de

Phone: $\quad$ +49(0) 40741051391

Postal Address: Institute of Experimental Cardiovascular Research University Medical Center Hamburg-Eppendorf (UKE) Martinistr. 52 20246 Hamburg 


\section{AFFIDAVIT}

Here I declare that my doctoral thesis entitled:

"Characterization of cardiac progenitor cell activity in engineered heart muscle"

has been written independently with no other sources and aids than quoted.

Elif Levent

Göttingen, April 2016 


\section{List of poster presentations}

- Elif Levent, Malte Tiburcy, Amke Hesse, Dörthe Katschinski, Mark Sussman and Wolfram-H Zimmermann (2013). Hypoxia/Reoxygenated engineered heart muscle as an injury model system to investigate cardiac progenitor cell function in heart repair in vitro. Cardiorepair European multidisciplinary initiative (CARE-MI) annual meeting Lisbon, Portugal.

- Elif Levent, Malte Tiburcy, Mark Sussman and Wolfram-H Zimmermann (2013). Engineered heart muscle as a model system to investigate cardiac progenitor cell function and heart regeneration in vitro. Deutsche Gesellschaft für Kardiologie (DGK) annual meeting, Mannheim, Germany.

- Elif Levent, Malte Tiburcy, Alberto Izarra, Antonio Bernad and Wolfram-H Zimmermann (2012). Engineered heart tissue to investigate cardiac progenitor cell function and heart regeneration in vitro. Cardiorepair European multidisciplinary initiative (CARE-MI) annual meeting, Leuven, Belgium.

\section{List of publications}

- Vogler M, Zieseniss A, Hesse AR, Levent E, Tiburcy M, Heinze E, Burzlaff N, Schley G, Eckardt KU, Willam C, Katschinski DM. (2015). Pre- and postconditional inhibition of prolyl-4-hydroxylase domain enzymes protects the heart from an ischemic insult. Pflugers Arch. 467:2141-9.

- Izarra A, Moscoso I, Levent E, Cañón S, Cerrada I, Díez-Juan A, Blanca V, Núñez-Gil IJ, Valiente I, Ruíz-Sauri A, Sepúlveda P, Tiburcy M, Zimmermann WH, Bernad A. (2014). mir-133a enhances the protective capacity of cardiac progenitors cells after myocardial infarction. Stem Cell Reports. 3:1029-42.

- Hesse $\mathrm{AR}^{\star}$, Levent $\mathbf{E}^{\star}$, Zieseniss A, Tiburcy M, Zimmermann WH, Katschinski DM. (2014). Lights on for HIF-1a: genetically enhanced mouse cardiomyocytes for heart tissue imaging. Cell Physiol Biochem. 34:455-62. (*equal contribution) 


\section{$\underline{\text { Table of Contents }}$}

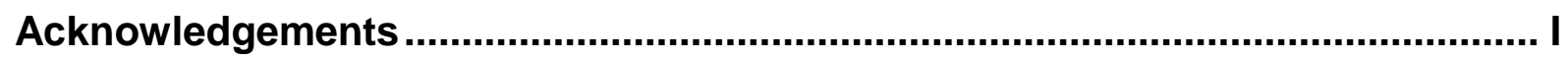

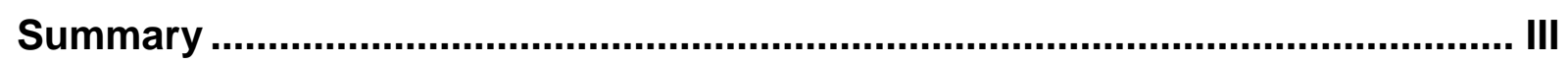

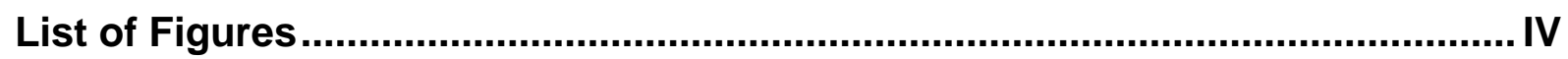

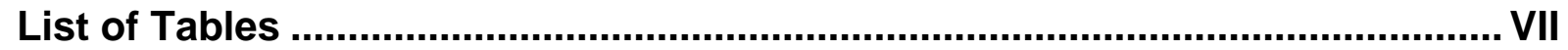

Abbreviations

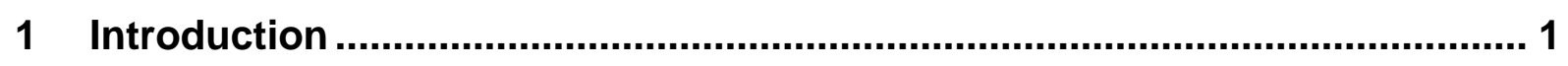

1.1 Evidence for cardiomyocyte renewal in the postnatal heart........................ 1

1.2 Origin of new cardiomyocytes in the postnatal heart ............................... 1

1.3 Stem/progenitor cell markers in the heart .............................................. 4

1.4 Evidence for cardiac progenitors in the adult heart................................. 6

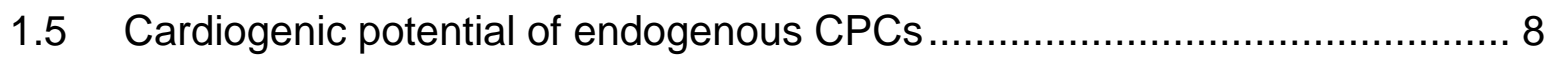

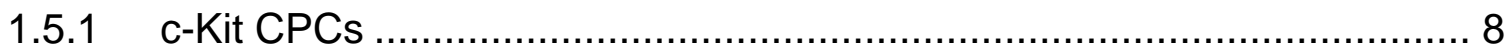

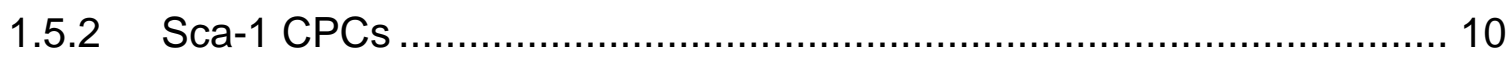

1.5.3 Vessel resident progenitor cells ................................................ 11

1.6 Exogenous regeneration by CPCs ………........................................ 13

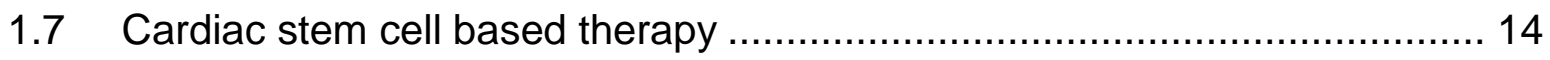

1.8 EHM as an in vitro cardiac muscle model............................................ 18

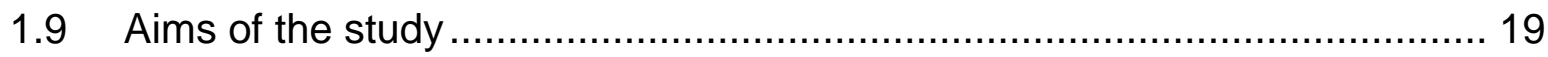




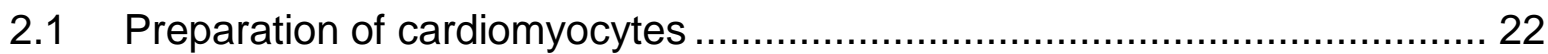

2.1.1 Neonatal rat cardiomyocytes...................................................... 22

2.1.2 Mouse embryonic stem cell-derived cardiomyocytes .......................... 22

2.1.3 Human embryonic stem cell-derived cardiomyocytes ......................... 24

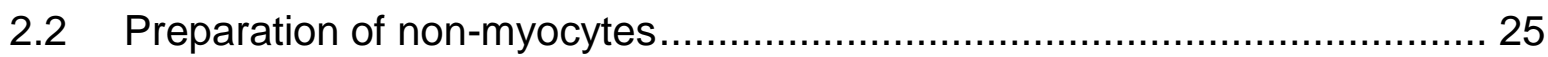

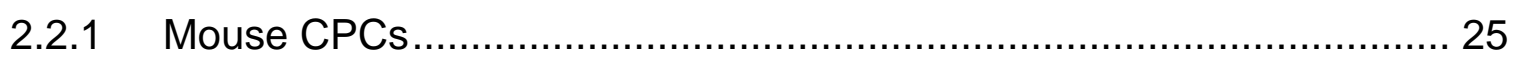

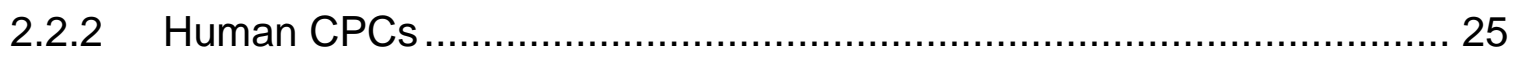

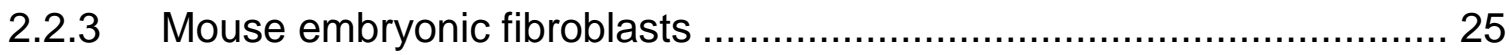

2.2.4 Human foreskin fibroblasts........................................................ 26

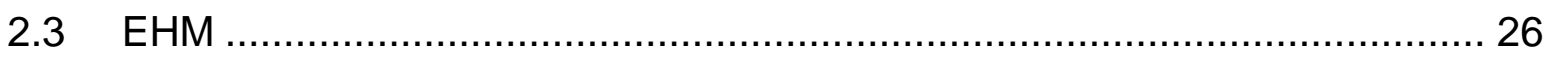

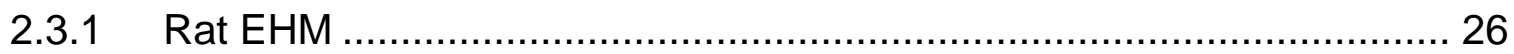

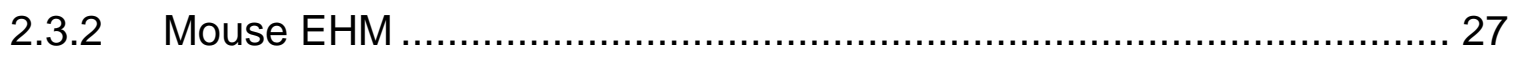

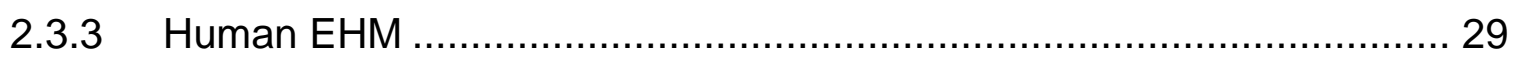

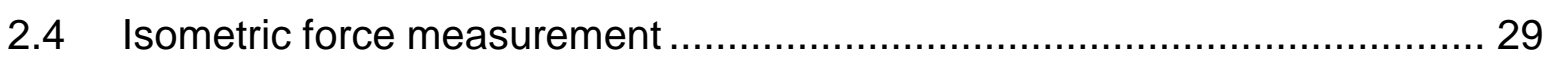

2.5 Immunostaining and microscopy ………........................................ 30

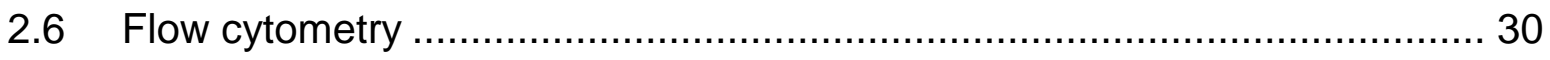

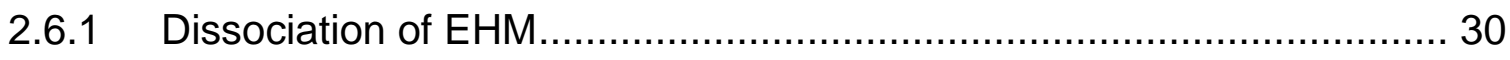

2.6.2 Immunostaining for intracellular antigens ......................................... 31

2.6.3 Immunostaining for cell surface proteins ......................................... 31 
2.6.4 Sorting of EHM-derived cells.

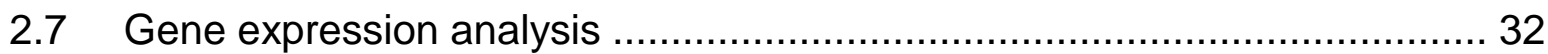

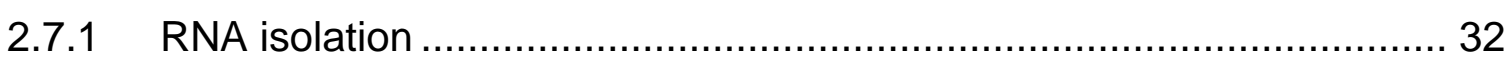

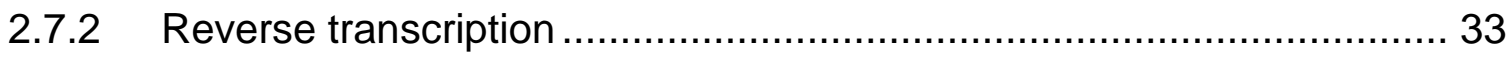

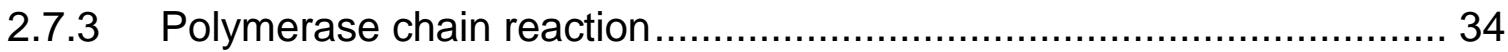

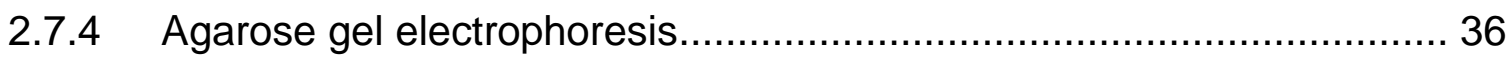

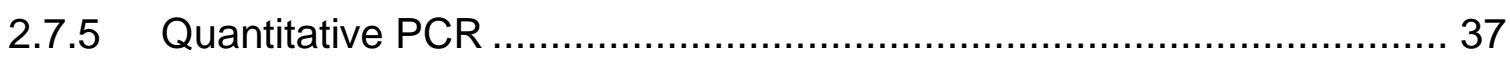

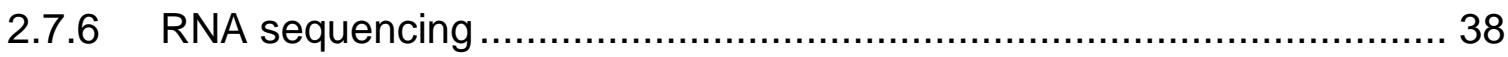

2.8 Cell based models of cardiomyocyte hypoxia....................................... 38

2.8.1 Imaging hypoxia in mouse ODD-Luc cardiomyocytes......................... 39

2.8.2 Imaging hypoxia in mouse ODD-Luc EHM ..................................... 39

2.8.3 Generation of ODD-Luc human embryonic stem cell line ................... 40

2.8.4 Hypoxia imaging in ODD-Luc human cardiomyocytes ......................... 41

2.8.5 Hypoxia/Reoxygenation injury in human EHM ................................ 41

2.8.6 Chronic hypoxia injury in human EHM .......................................... 42

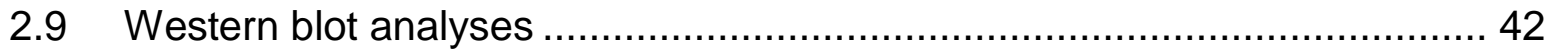

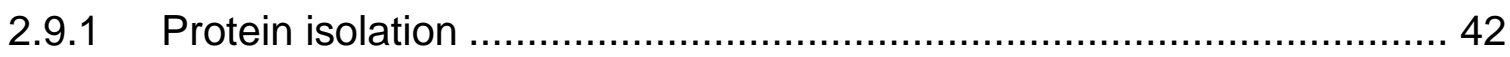

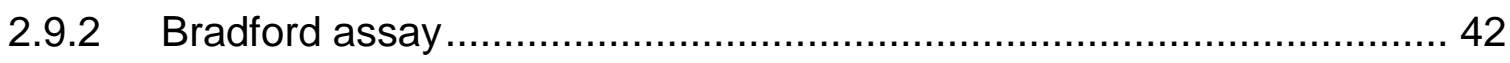

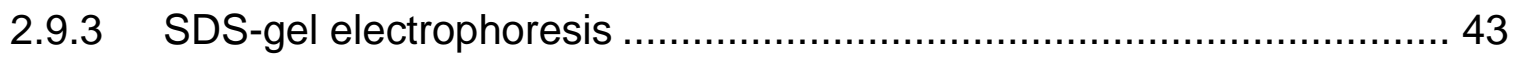

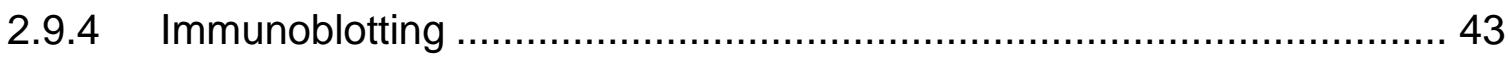


2.10 Quantification of lactate release

2.11 Quantification of intracellular ATP

2.12 Statistics

3 Results 46

3.1 Characterization of CPCs in monolayer culture 46

3.1.1 Mouse CPCs show a mesenchymal phenotype 46

3.1.2 Human CPCs are morphologically distinct from fibroblasts 47

3.1.3 Human CPC pools contain mesenchymal/endothelial cells 48

3.1.4 Human CPCs exhibit a unique transcriptome profile 51

3.2 Cardio-supportive effects of mouse CPCs in EHM 55

3.2.1 CPCs support functional maturation of EHM 55

3.3 Enhanced paracrine support by genetic manipulation of mouse CPCs ....... 58

3.4 Human EHM model 61

3.4.1 Contractile performance in human EHM 63

3.4.2 Passive biomechanical properties in human EHM 64

3.4.3 Cardiomyocyte structure and function in human EHM 65

3.4.4 Human CPC retention in EHM 68

3.4.5 No evidence of cardiomyocyte transdifferentiation in human EHM . 69

3.5 Modeling hypoxic injury in EHM. 72

3.5.1 Hypoxia response in human cardiomyocytes 72 
3.5.2 Hypoxia response in human EHM.

3.5.3 Hypoxia/Reoxygenation damage in human EHM............................. 74

3.6 Assessment of the protective effects of human CPCs in EHM ...................76

3.6.1 No evidence for paracrine protection by CPCs ................................ 77

3.6.2 Discrimination of paracrine effects by transcriptome profiling .............. 80

3.6.3 Cardio-protective effects of CPCs in EHM exposed to chronic hypoxia 82

3.7 Development of a genetic hypoxia indicator model 84

3.7.1 Hypoxia sensing in ODD-Luc mouse cardiomyocytes 84

3.7.2 Hypoxia sensing in ODD-Luc mouse EHM 87

3.7.3 Hypoxia sensing in human cardiomyocytes 87

4 Discussion 89

4.1 Characterization of CPCs 90

4.2 Human CPCs might have an epicardial origin 92

4.3 CPCs and fibroblasts support EHM formation 93

4.4 CPCs do not contribute to cardiac homeostasis under normal conditions ... 94

4.5 CPCs can be modified to enhance their tissue-supporting activity 94

4.6 Development of EHM models of hypoxic damage .................................. 95

4.7 CPCs protect EHM under chronic hypoxia …....................................... 96

4.8 Live cell imaging of cardiomyocyte oxygenation..................................... 96 


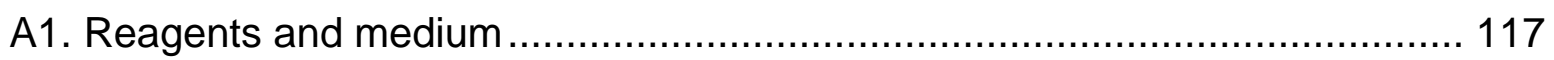

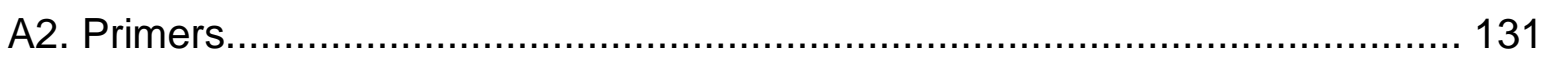

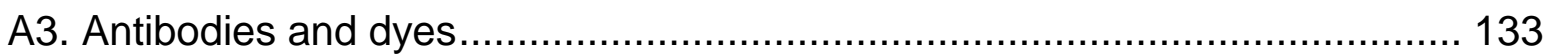

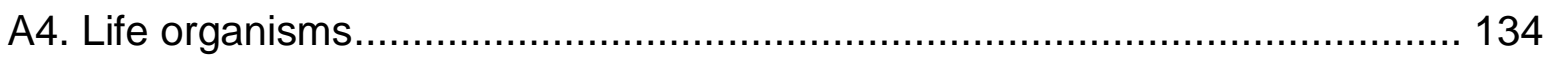

A5. Human CPC specific differentially expressed genes................................. 135

A6. Growth factors and cytokines differentially expressed in hCPCs and hFFs .. 146 


\section{Acknowledgements}

I would like to present my gratitude firstly to my supervisor Prof. Wolfram-Hubertus Zimmermann for giving me the opportunity to work on this project and the valuable advices which he has given during the data discussions throughout my PhD.

I would like to furthermore thank my thesis committee members Prof. Dr. Dörthe Katschinski and Prof. Dr. Viacheslav Nikolaev for their persistent support and guidance during my study.

I am especially grateful to Dr. Malte Tiburcy for the continuous and firsthand support and advice he has given me throughout my project. I would like to also thank him for giving me the freedom to develop my own ideas and continuously being open to my questions. I will also not forget our warm and friendly group meetings in the Christmas market. Moreover, I would like to thank Andreas Schraut for generating cardiomyocyte specific antibiotically selectable ODD-Luc expressing mESC cell line, Dr. Claudia Noack for establishing the ODD-Luc construct, Mrs. Krasimira Sharkova for her help in the generation of the ODD-Luc expressing hES2 cell line as well as her sincere friendship and Dr. Sebastian Zeidler for his patience and support with the analysis of RNAseq data. Also, I thank to Daria Reher for constantly providing very valuable cardiomyocytes.

I have spent this long period together with my very nice and valuable friends that I feel really lucky to have met them. Foremost, I would like to express my sincere gratitude for having my close friend, Eriona Heta nearby. She shared all my moods and kept me alive with her support. I especially thank her for having the door of her home always open to me, no matter what time it is. I felt warmer and relieved with the support of her presence. Furthermore, I thank to my lab mates; Satish Galla, Farah Raad, Sumon Sur, Elena Pavlova and Dr. Poh Loong Soong for their sincere friendships. I enjoyed the lab and the office a lot more together with them. Having many years past together, I am very thankful for their presence and giving the spiritual support.

I think it was magic to have spent four years of my PhD with my old friend, Mine Bakar. Her presence was very important and a big support for me throughout the 
duration of my PhD. I became free from all the concerns and enjoyed my time here a lot more together with her.

Lastly, I would like to express my special gratitude to my family. My parents and my brother were all the time nearby me. I would like to thank them a lot for supporting me to come here and their constant belief in me throughout all these years. 


\section{Summary}

The heart has been considered a post-mitotic organ incapable of regeneration upon injury. Recent findings suggest that the heart contains cardiac progenitor cells (CPCs) with the potential to give rise to cardiovascular cells. CPCs are currently under clinical investigation aiming at cell-based induction of heart regeneration in patients with myocardial infarction related injury. The mechanisms of action underlying the reported beneficial effects of CPCs remain for the most part elusive. This study was designed to enhance our knowledge on CPC biological activity. By making use of engineered heart muscle (EHM) constructed from cardiomyocytes, fibroblasts and CPCs in a collagen type 1 hydrogel the aim was to simulate a threedimensional heart muscle environment as closely as possible. Different types of mouse and human CPCs were investigated and found to be mesenchymal cells distinct from fibroblasts. Transcriptome profiling suggested a pericyte phenotype within the human CPC population. Despite the apparent differences in cell phenotype, CPCs and fibroblast supported the assembly of cardiomyocytes into macroscopically contracting EHM. Evidence for CPC transdifferentiation in EHM could not be obtained. Novel EHM models of hypoxia/reoxygenation and chronic hypoxia damage were developed and used to study potential cardio-protective effects of CPCs. Surprisingly, these experiments revealed that hypoxia/reoxygenation damage could be attenuated by fibroblasts, but not by CPCs. This effect appeared to be mediated by the release of cell protective growth factors and cytokines from fibroblasts. Conversely, transcriptome profiling suggested angiogeneic and immune modulatory activity in CPCs, which may not be effective in a vascular and leukocytefree EHM. The cell context specific biological activity of CPCs was further exemplified by studies in EHM tri-cultures composed of cardiomyocytes, fibroblasts and CPCs. Only tricultures with CPCs were protected from chonic hypoxia. Finally, to in the future be able to visualize the oxygenation level in cardiomyocytes, a transgenic hypoxia reporter was established. In summary, CPCs exhibited a distinct phenotype from fibroblasts. It appeared that CPCs require a specific mutlicellular context to exhibit protective effects upon hypoxia. EHM-hypoxia injury tools and a transgenic hypoxia reporter were developed to facilitate future organoid studies on cardioprotection. 


\section{List of Figures}

Figure 1. Heart regenerative response in mammalian heart

Figure 2. Schematic representation of c-Kit receptor structure and activation. 4

Figure 3. Hematopoietic stem cells in bone marrow niche and their activation. 6

Figure 4. Cardiac progenitor cells residing in the adult heart. 7

Figure 5. c-Kit cell progeny in the adult heart. 9

Figure 6. Unique and shared properties of pericytes and mesenchymal stem cells. 12

Figure 7. Schematic overview of the spatial distribution of putative CPCs. 13

Figure 8. Clinically tested cell sources for heart regeneration. 15

Figure 9. Schematic overview of potential mechanisms involved in heart repair upon CPC transplantation in vivo.. 18

Figure 10. Cardiac differentiation of mESCs. 23

Figure 11. Construction of rat EHM. 27

Figure 12. Casting mold for mouse and human EHM. 28

Figure 13. Bioluminescent reaction catalyzed by firefly luciferase. 39

Figure 14. ODD-Luc hES cells generated using TALEN technology.. 41

Figure 15. Lactic acid catalyzing reaction 44

Figure 16. Characterization of mCPCs. 47

Figure 17. Morphologically distinct phenotypes in hCPC and hFF monolayer cultures. 48

Figure 18. Expression of cardiac cell markers in hCPCs. 49 
Figure 19. Flow cytometry characterization of hCPCs. 50

Figure 20. Comparative transcriptome analysis. 52

Figure 21. Expression profile of cardiac cell-specific markers. 54

Figure 22. EHM structure and function enhanced by mCPCs. 56

Figure 23. Functional comparison of mCPC- and mEF-EHMs. 57

Figure 24. Purification of neonatal rat cardiomyocytes. 59

Figure 25. miR-133a enhanced paracrine activity of mCPCs.. 61

Figure 26. Defined hEHM model composed of $\mathrm{RFP}^{+}$-hCMs and GFP ${ }^{+}$-non-myocytes.

Figure 27. Cell distribution and cardiomyocyte morphology in hEHM. 63

Figure 28. hCPCs and hFFs supported EHM.. 64

Figure 29. Passive mechanical properties of hEHM. 65

Figure 30. Cardiomyocyte amount and phenotype in hEHM. 67

Figure 31. Retention of non-myocytes in hEHM. 69

Figure 32. Strategies to investigate cardiac differentiation of hCPCs in EHM. 71

Figure 33. Metabolic adaptation of human cardiomyocytes under hypoxia. 73

Figure 34. Lactate production by hEHM cultured under normoxia and hypoxia. 74

Figure 35. Development of a hypoxia/reoxygenation injury hEHM model. 75

Figure 36. Validation of the hypoxia/reoxygenation injury hEHM model.. 76

Figure 37. Effects of conditioned medium on EHM function upon H/R injury. 78 
Figure 38. Effects of conditioned medium on EHM cardiomyocyte content upon $\mathrm{H} / \mathrm{R}$ injury. 79

Figure 39. Impaired $\beta$-adrenergic signaling in $\mathrm{hEHM}$ after $\mathrm{H} / \mathrm{R}$ injury. 79

Figure 40. Paracrine activity in hCPCs and hFFs. 81

Figure 41. Effects of CPC in EHM tricultures upon chronic hypoxia. 83

Figure 42. Enhanced cardiomyocyte survival and function in EHM tri-cultures 84

Figure 43. Validation of ODD-Luc hypoxia reporter in cardiomyocyte culture. 86

Figure 44. Hypoxia and repoxygenation responses in ODD-Luc EHM. 87

Figure 45. Hypoxia response in ODD-Luc human cardiomyocytes. 88 


\section{List of Tables}

Table 1. Putative CPCs and their phenotype in the adult heart. ............................. 8

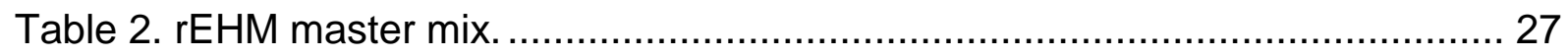

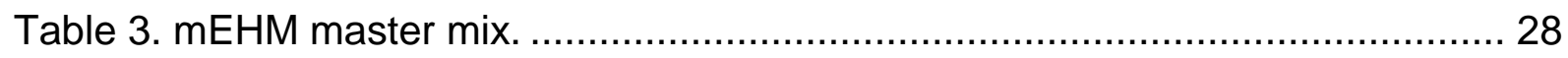

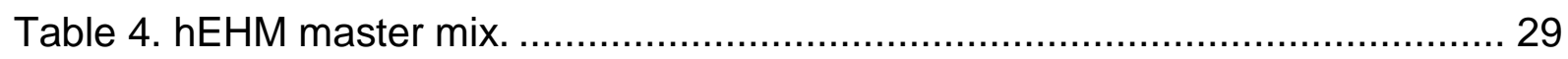

Table 5. Composition of DNase treatment......................................................... 33

Table 6. Composition of cDNA synthesis reaction............................................. 33

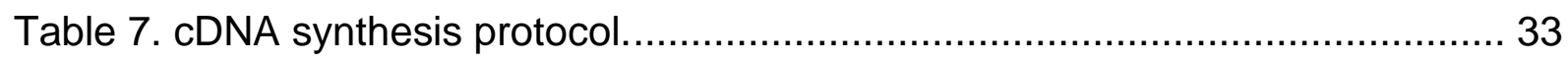

Table 8. Composition of the PCR reaction for all targets without CD31 ................. 34

Table 9. Composition of the PCR reaction for CD31............................................ 35

Table 10. PCR program for FGF-2/VEGF-A/PDGF/GAPDH. .............................. 35

Table 11. PCR program for IGF-1/GATA4/ACTC1/CTnI. ..................................... 35

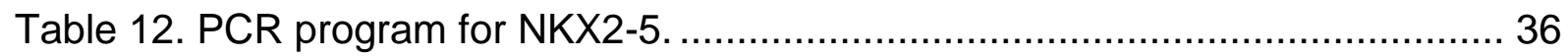

Table 13. PCR program for CD31/c-Kit. ........................................................... 36

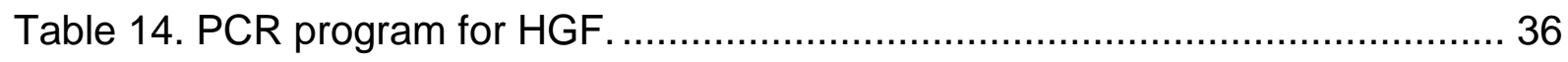

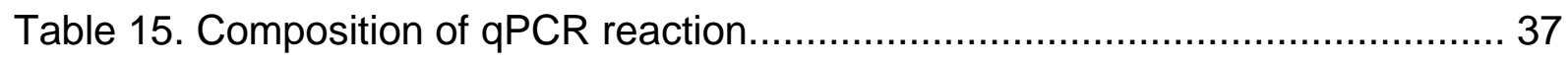

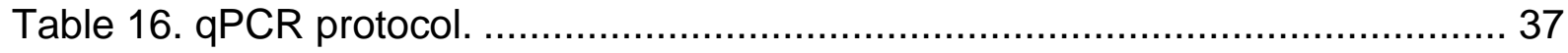

Table 17. Differentially expressed plasma membrane genes in hCPCs compared to

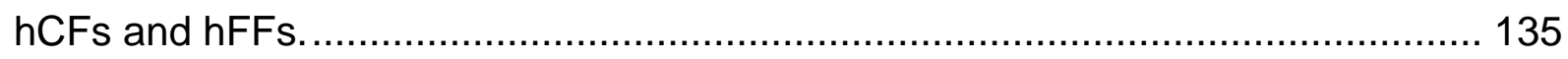

Table 18. Differentially expressed cell adhesion genes in hCPCs compared to hCFs and hFFs. 
Table 19. Differentially expressed genes involved in actin cytoskeleton organization in hCPCs compared to hCFs and hFFs.

Table 20. Differentially expressed extracellular region genes in hCPCs compared to hCFs and hFFs.

Table 21. hCPC specific up-regulated growth factors and cytokines compared to hFFs.

Table 22. hFF specific up-regulated growth factors and cytokines compared to hCPCs. 


\section{Abbreviations}

\begin{tabular}{|c|c|}
\hline ACE & Angiotensin converting enzyme \\
\hline APS & Ammonium persulfate \\
\hline ACTA2 & Actin, alpha 2, smooth muscle \\
\hline ALCADIA & $\begin{array}{l}\text { Autologous human cardiac-derived stem cell to treat } \\
\text { ischemic cardiomyopathy }\end{array}$ \\
\hline ARHGAP26 & Rho GTPase Activating Protein 26 \\
\hline ARHGEF2 & Rho/Rac Guanine Nucleotide Exchange Factor (GEF) 2 \\
\hline BrdU & Bromodeoxyuridine \\
\hline BM & Bone marrow \\
\hline BM-MSC & Bone marrow-derived mesenchymal stem cell \\
\hline BM-MNC & Bone marrow-derived mononuclear cells \\
\hline BSA & Bovine serum albumin \\
\hline CPC & Cardiac progenitor cell \\
\hline CADUCEUS & $\begin{array}{l}\text { Cardiosphere-derived autologous stem cells to reverse } \\
\text { ventricular dysfunction }\end{array}$ \\
\hline CDC & Cardiosphere-derived cell \\
\hline $\mathrm{CM}$ & Cardiomyocytes \\
\hline C & Carbon \\
\hline cDNA & Complementary DNA \\
\hline $\mathrm{CDH} 5$ & Cadherin 5, type 2 (Vascular endothelium) \\
\hline CHIR & Glycogen synthase kinase $3 \beta$ inhibitor \\
\hline CMR & Custom made replacement \\
\hline Cacl2 & Calcium chloride \\
\hline cTnT & Cardiac troponin $\mathrm{T}$ \\
\hline
\end{tabular}


DNA Dioxyribonucleic acid

DEG Differentially expressed gene

DDR2 Discoidin domain-containing receptor 2

DMSO Dimethyl sulfoxide

EHM Engineered heart muscle

ECM Extracellular matrix

EB Embryoid body

EDTA Ethylenediaminetetraacetic acid

\begin{tabular}{ll} 
FA & Formaldehyde \\
\hline FGF-2 & Basic fibroblast growth factor \\
\hline FOC & Force of contraction \\
\hline GATA4 & GATA binding protein 4 \\
\hline GFP & Green fluorescent protein \\
\hline HGF & Hepatocyte growth factor \\
\hline HIF-1a & $\begin{array}{l}\text { Hypoxia Inducible Factor 1, Alpha Subunit (Basic Helix-Loop- } \\
\text { Helix Transcription Factor) }\end{array}$ \\
\hline hCM & Human cardiomyocyte \\
\hline H/R & Hypoxia/Reoxygenation \\
\hline HEK & Human embryonic kidney \\
\hline hCPC & Human cardiac progenitor cell \\
\hline hEHM & Human engineered heart muscle \\
\hline IGF-1 & Insulin-like growth factor-1 \\
\hline IWP4 & Wnt/ $\beta$-catenin pathway antagonist \\
\hline
\end{tabular}




\begin{tabular}{|c|c|}
\hline IFN-Y & Interferon-gamma \\
\hline ITS-X & Insulin-Transferrin-Selenium-Ethanolamine \\
\hline Ki67 & Marker of proliferation Ki-67 \\
\hline KIT (CD117 or c-kit) & $\begin{array}{l}\text { V-Kit Hardy-Zuckerman } 4 \text { Feline Sarcoma Viral Oncogene } \\
\text { Homolog }\end{array}$ \\
\hline KDR & Kinase insert domain receptor \\
\hline KSR & Knockout serum replacement \\
\hline $\mathrm{KO}$ & Knockout \\
\hline $\mathrm{KCl}$ & Potassium chloride \\
\hline LVAD & Left ventricular assist device \\
\hline LV & Left ventricular \\
\hline Luc & Firefly luciferase \\
\hline LDH & Lactate dehydrogenase \\
\hline LIF & Leukemia inhibitory factor \\
\hline MADM & Mosaic analysis with double markers \\
\hline MEF2 & Myocyte enhancer factor 2 \\
\hline MDR1 & Multi drug resistance protein 1 \\
\hline MSC & Mesenchymal stem cell \\
\hline MAPK & Mitogen activated protein kinase \\
\hline $\mathrm{mCPC}$ & Mouse cardiac progenitor cell \\
\hline MEF & Mouse embryonic fibroblast \\
\hline $\mathrm{MHC}$ & Myosin heavy chain \\
\hline $\mathrm{mCM}$ & Mouse cardiomyocyte \\
\hline
\end{tabular}




\begin{tabular}{|c|c|}
\hline MYH11 & Myosin heavy chain 11, smooth muscle \\
\hline $\mathrm{mCF}$ & Mouse cardiac fibroblast \\
\hline MAPK & Mitogen activated protein kinase \\
\hline $\mathrm{MgCl} 2$ & Magnesium chloride \\
\hline Nkx2.5 & NK2 transcription factor related, locus 5 \\
\hline NM & Non-myocyte \\
\hline NRCM & Neonatal rat cardiomyocyte \\
\hline $\mathrm{NADH}$ & Nicotinamide adenine dinucleotide \\
\hline NES & Nestin \\
\hline NEAA & Non-essential amino acid \\
\hline $\mathrm{NaHCO} 3$ & Sodium bicarbonate \\
\hline $\mathrm{NaH} 2 \mathrm{PO} 4$ & Sodium phosphate monobasic \\
\hline ODD & Oxygen dependent degradation domain of HIF-1a \\
\hline PDGFRA & Platelet-derived growth factor receptor, alpha polypeptide \\
\hline PECAM1 & Platelet enodethelial cell adhesion molecule-1 \\
\hline PHD2 & Prolyl-hydroxylase domain-containing protein 2 \\
\hline PFA & Paraformaldehyde \\
\hline POSTN & Periostin, Osteoblast specific factor \\
\hline PDGFRB & Platelet-derived growth factor receptor, beta polypeptide \\
\hline PHD2 & Prolyl-hydroxylase domain-containing protein 2 \\
\hline PBS & Phosphate buffered saline \\
\hline PDGFA & Platelet-derived growth factor, alpha polypeptide \\
\hline
\end{tabular}




\begin{tabular}{|c|c|}
\hline PDGF & Platelet-derived growth factor \\
\hline RFP & Red fluorescent protein \\
\hline RoT & Room temperature \\
\hline $\operatorname{ReT}$ & Resting tension \\
\hline RNA & Ribonucleic acid \\
\hline Rho & Ras homology \\
\hline RAC2 & Rho Family, Small GTP Binding Protein \\
\hline Sca-1 & Stem cells antigen -1 \\
\hline SMA & Smooth muscle actin \\
\hline SF & Serum free \\
\hline SDS & Sodium dodecyl sulfate \\
\hline TLN1 & Talin 1 \\
\hline TGF-B1 & Transforming growth factor-beta 1 \\
\hline tdRFP & Tandem dimer red fluorescent protein \\
\hline T3 & Triiodothyronine \\
\hline TAGLN & Transgelin \\
\hline TCF21 & Transcription factor 21 \\
\hline TEMED & Tetramethylethylenediamine \\
\hline vWF & Von Willebrand factor \\
\hline VEGFA & Vascular endothelial growth factor a \\
\hline WIPF1 & WAS/WASL interacting protein family, member 1 \\
\hline WT1 & Wilms tumor 1 \\
\hline
\end{tabular}




\section{Introduction}

Myocardial infarction causes the loss of approximately 1 billion cardiomyocytes, which are not regenerated, but replaced by non-contractile scar tissue (Gerbin and Murry 2015). The associated loss of function is typically at first compensated by a hypertrophic response, which finally results in additional cardiomyocyte death and whole organ failure (Mill et al. 2011, Zangi et al. 2013). Therapeutic strategies to reduce cardiomyocyte death, regenerate the infarcted myocardium and prevent progression to heart failure are clearly needed in light of the high mortality in affected patients (Yancy et al. 2013).

\subsection{Evidence for cardiomyocyte renewal in the postnatal heart}

The embryonic heart grows through proliferation of cardiomyocytes. Fetal cardiomyocytes in the human heart start to withdraw from the cell cycle. Shortly after birth, most of the cardiomyocytes are considered post-mitotic; subsequent cardiomyocyte growth is by hypertrophy (Laflamme and Murry 2011). Over the recent years, a number of studies suggested de novo cardiomyogenesis challenging the view that the heart is a static organ (Raphael Rubin 2008). Elegant carbon dating studies in human hearts identified very low DNA-replication (lifetime average of $<1 \% /$ year), arguing against significant self-renewal of the heart (Bergmann et al. 2009, Bergmann et al. 2015). Cardiomyocytes shortly after birth showed the highest cell cycle activity, which then decreases with age (Bergmann et al. 2015, Senyo et al. 2013).

\subsection{Origin of new cardiomyocytes in the postnatal heart}

Identification of cycling cardiomyocytes or progenitors with cardiomyogenic potential in the adult heart would open the door for targeted stimulation of these cells. In lower vertebrates, such as zebrafish and neonatal mice (1-day old), the heart is capable of fully regenerating, which is mediated by the proliferation of pre-existing cardiomyocytes (Porrello et al. 2011). Given the fact that the regenerative response declines in the adult mammalian heart (Figure 1) (Laflamme and Murry 2011), reactivation of proliferation in endogenous cardiomyocytes appears attractive to 
achieve myocardial remuscularization (Bersell et al. 2009, Engel et al. 2006, Hassink et al. 2008, Kuhn et al. 2007).

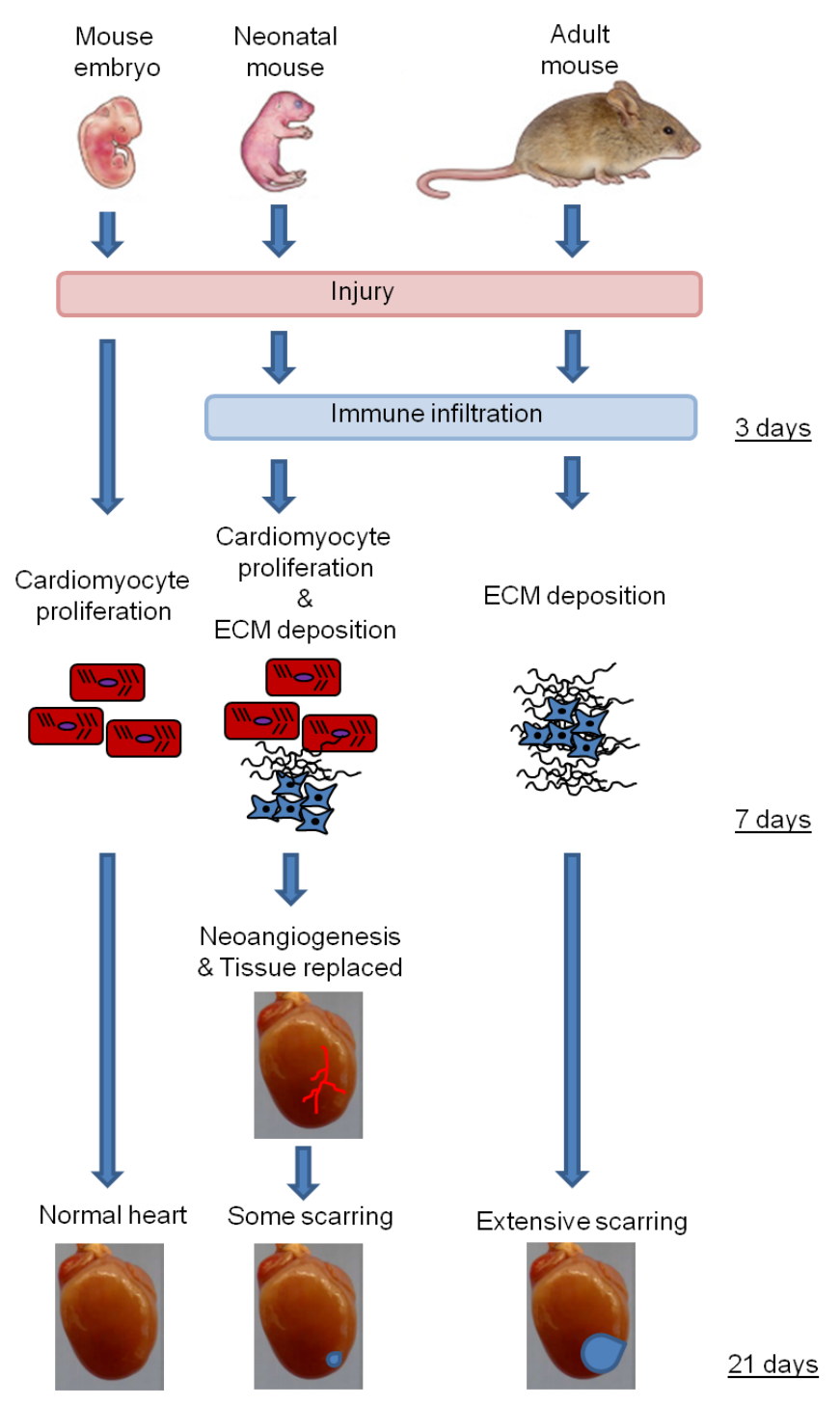

Figure 1. Heart regenerative response in mammalian heart. Embryonic stage: cardiomyocytes undergo cell-cycle entry and repopulate the heart. Neonatal stage: cardiomyocyte proliferation and angiogenesis as the two main mechanisms involved in complete regeneration of neonatal mouse heart following injury. Adult stage: insufficient cardiomyocyte proliferation and extracellular matrix deposition after injury. Schematic adapted from Uygur et al. (2016).

Genetic fate mapping strategies based on the expression of fluorescence reporter genes in specific cell types are powerful tools to track the origin of new cardiomyocytes formed during physiological and pathological conditions (Hsieh et al. 2007). Differential labeling of endogenous cardiomyocytes at a given time point indicated no change in the percentage of pre-existing cardiomyocytes during physiological aging, suggesting that non-cardiomyocytes do not contribute to heart development under normal conditions (Hsieh et al. 2007). Evidence for DNAreplication and cell cycle activity in resident adult cardiomyocytes (genetically labeled) (Malliaras et al. 2013, Senyo et al. 2013) and unambiguously labeling of dividing cardiomyocytes with alternative fate-mapping approaches, such as mosaic analysis with double markers (MADM) (Ali et al. 2014), supported the hypothesis that endogenous cardiomyocytes are the main source of cardiomyocyte turnover under physiological conditions, albeit in a very low amount ( $0.1 \%$ of total cardiomyocytes) (Ali et al. 2014, Malliaras et al. 2013, Senyo et al. 2013). Collectively, there is 
compelling evidence for postnatal cardiomyogenesis via proliferation of endogenous cardiomyocytes; however, these rare events cannot be considered of relevance for myocardial regeneration after injury of the adult heart.

Whether endogenous cardiomyocyte proliferation or remuscularization via progenitor cell activation is enhanced under pathological conditions remains a matter of debate. Studies, which utilized fate-mapping strategy to label pre-existing cardiomyocytes as previously described, demonstrated a dilution in the percentage of labeled preexisting cardiomyocytes in the infarcted region of the heart after myocardial infarction (Ellison et al. 2013, Hsieh et al. 2007). In addition, Malliaras et al. reported that although adult cardiomyocyte cycling increases after myocardial injury, the majority of likely proliferating cardiomyocytes are from another cell source, so called progenitor cells. Thus, both endogenous cardiomyocytes and cardiac progenitors appeared to be involved in the replacement of lost cardiomyocytes (Malliaras et al. 2013). Although these studies provided some indirect evidence for new myocyte formation from a progenitor source, there is no consensus in the literature on the involved mechanisms. For example, in a recent study mass spectrometric analysis of cardiomyocytes labeled with ${ }^{15} \mathrm{~N}$-stable isotope revealed that new myocytes were mostly originating from adult cardiomyocytes after myocardial infarction, although a minor contribution of progenitors could not be excluded (Senyo et al. 2013).

The discrepancies between different studies might be due to technical reasons. Fatemapping strategies of resident cardiomyocytes (Ellison et al. 2013, Hsieh et al. 2007, Malliaras et al. 2013) can only provide indirect evidence for cardiac differentiation of endogenous progenitor cells. In addition, the number of the cells analyzed by multiisotope imaging mass spectrometry (Senyo et al. 2013) may have been too small to define the contribution of progenitor-derived cardiomyocytes. The limitation of these different techniques could only be addressed by direct and unambiguous labeling of specific progenitors and their progeny in vivo. 


\subsection{Stem/progenitor cell markers in the heart}

c-Kit, also known as v-kit hardy-zuckerman 4 feline sarcoma viral oncogene homolog receptor function has been shown to play a crucial role for the migration, proliferation and survival of primordial germ cells, neural-crest-derived melanoblasts and hematopoietic precursors during embryo development. Many other organs and systems (e.g. skeleton, tooth, brain and neural tube, sensory organs, the respiratory system, the digestive system, endocrine organs, the genitoexcretory system and circulatory system) have been also found to express c-Kit, but without being essentially required for their development (Bernex et al. 1996).

C-Kit receptor belongs to the family of type III receptor tyrosine kinase (RTK), which also includes platelet-derived growth factor receptor (PDGFR), the macrophage colony stimulating factor receptor (CSFR) and fms related tyrosine kinase 3 (FLT3). c-Kit receptor tyrosine kinase structure is characterized by extracellular domain comprised of five immunoglobulin-like domains, spanning transmembrane region followed by an intracellular part that contains juxtamembrane, tyrosine kinase domain 1 and 2, which is split by a kinase insert sequence and carboxyterminal tail (Figure 2) (Lennartsson and Ronnstrand 2012).
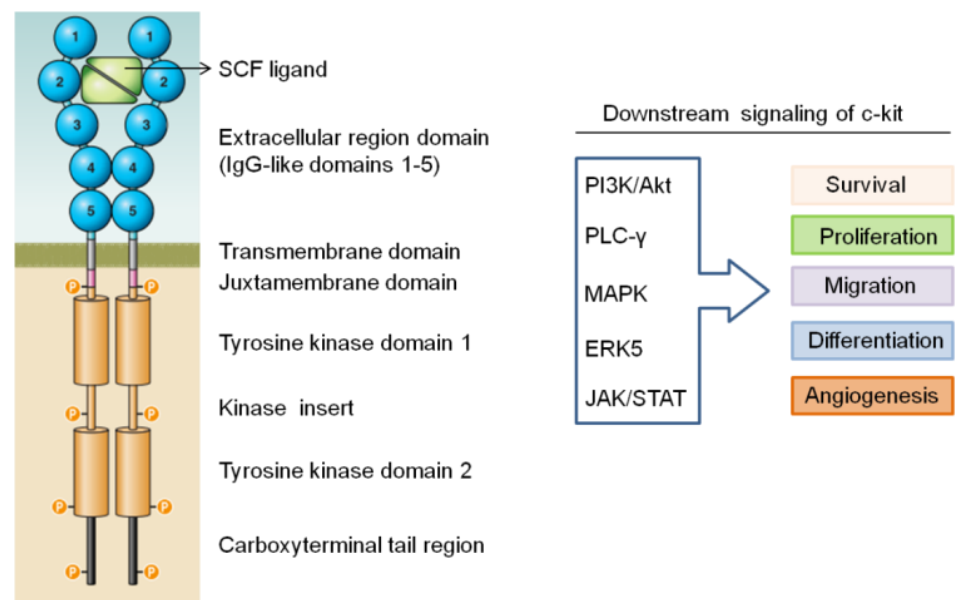

Figure 2. Schematic representation of c-Kit receptor structure and activation. Stem cell factor (SCF) ligand binding and dimerization of $\mathrm{c}$-Kit followed by autophopshorylation on tyrosine residues and activated downstream signaling pathways. Schematic adapted from Lennartsson et al. (2012). 
Binding of c-Kit ligand, stem cell factor (SCF) triggers dimerization of c-Kit receptor followed by autophosphorylation of the receptor. Highly phosphorylated tyrosine residues on the activated c-Kit receptor interact with different Src homology domain 2 (SH2) or SH3 domain containing adaptor proteins which subsequently coordinate the activation of downstream signaling pathways including phosphotidylinositol 3-kinases (PI3K)/AKT, phospholipase C (PLC)- $\mathrm{y}$, mitogen-activated protein kinases (ERK1/2, p38, JNK), ERK5 and Janus kinase (JAK)/Signal tansducer and activator of transcription (STAT) signal transduction pathways that are involved in cell survival, proliferation, migration, differentiation, and angiogenesis (Liang et al. 2013).

Stem cells antigen-1 (Sca-1) is a member of lymphocyte activation protein-6A (Ly-6A) gene family and is a glycosylphosphatidylinositol-anchored cell surface protein (GPIAP) (van de Rijn et al. 1989). The ligand for Sca-1 has been not characterized yet; however there is evidence that Sca-1 is associated with Src family kinase members suggesting that Sca-1 functions as a receptor (Stefanova et al. 1991). Sca-1 expression has been detected in several organs; mostly restricted to endothelial cells or vasculature in the heart, brain and liver, in cortical tubes of kidney as well as in thymus and spleen (van de Rijn et al. 1989). It is important to note that although Sca1 protein has been reported in mouse, a human Sca-1 analogue has not been identified yet. Sca- $1^{+}$cells in human are considered Sca- $1^{+}$-like cells that have been isolated from the adult human heart based on an anti-mouse Sca-1 antibody (Valente et al. 2014).

c-Kit and Sca-1 are two surface proteins reported to be expressed on hematopoietic stem cells (HSCs) that show the capacity of self-renewing, giving rise to committed progenitors and differentiating into all cell lineages of blood system (Ikuta and Weissman 1992, Ito et al. 2003). HSCs are mainly found inside specific microenvironments, so called endosteal niche in the bone marrow within the trabecular bone. The C-Kit receptor ligand, SCF and C-X-C motif chemokine 12 (CXCL12; also known as stroma cell-derived factor 1 [SDF-1]) are secreted by osteoblasts, mesenchymal stromal cells/fibroblasts and CXCL12-abundant reticular (CAR) cells within the endosteal niche to support long-term maintenance of HSCs (Figure 3). Furthermore, c-Kit and Sca-1 expression on HSCs and their multipotent progenitors (e.g. myeloid and lymphoid progenitors) suggest that these receptors are 
important for self-renewal, motility and differentiation of the stem/progenitor cells during hematopoiesis (Ito et al. 2003, Wilson et al. 2007).

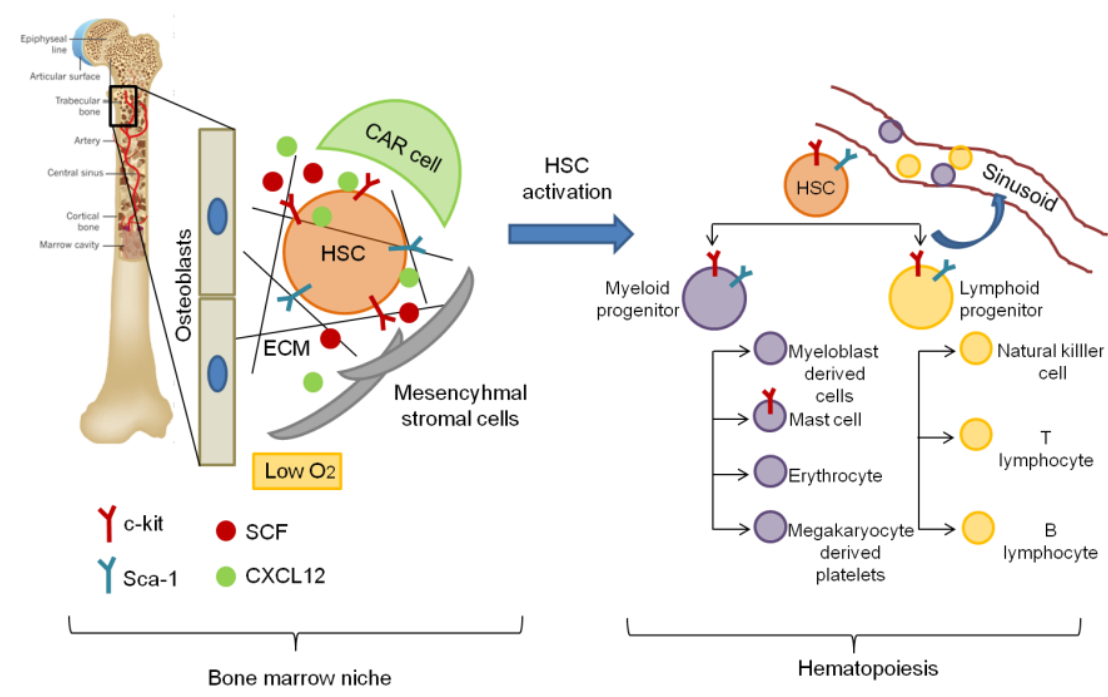

Figure 3. Hematopoietic stem cells in bone marrow niche and their activation. Schematic adapted from Morrison et al. (2014).

\subsection{Evidence for cardiac progenitors in the adult heart}

The first evidence for the existence of stem cells in the adult heart was obtained two decades ago in the rat heart with isolation of cells that display mesenchymal stem cell (MSC) characteristics differentiating into mesodermal lineages (Warejcka et al. 1996). Subsequently, cells decorated with c-Kit and Sca-1 protein were identified in the heart as putative cardiac stem/progenitor cells from the adult heart (Beltrami et al. 2003, Oh et al. 2003). Resident cardiac progenitor cells (CPCs) were firstly identified based on the expression of tyrosine kinase receptor, c-Kit. Isolated $\mathrm{c}-\mathrm{Kit}^{+}$cardiac cells showed partial expression of cardiac transcription factors NK2 homeobox 5 (Nkx2.5), GATA binding protein 4 (GATA4) and myocyte enhancer factor 2 (MEF2), suggesting the presence of a small proportion (7-10\%) of cardiac committed progenies. They displayed stem cell characteristics, i.e., clonogenic growth, selfrenewing capacity and multipotency with evidence presented for their differentiation into cardiomyocytes ( $\alpha$-sarcomeric actin ${ }^{+}$, cardiac myosin heavy chain ${ }^{+}$), smooth muscle cells ( $\alpha$-smooth muscle actin ${ }^{+}$), and endothelial cells (von Willebrand factor ${ }^{+}$) within 7-10 days under defined medium conditions in vitro (Beltrami et al. 2003). In 
addition, direct fluorescence labeling of isolated and in vitro expanded CPCs demonstrated that they were able to contribute new myocardium formation upon injection into the infarcted region of the heart, giving rise to all three cardiac cells mentioned above (Beltrami et al. 2003).

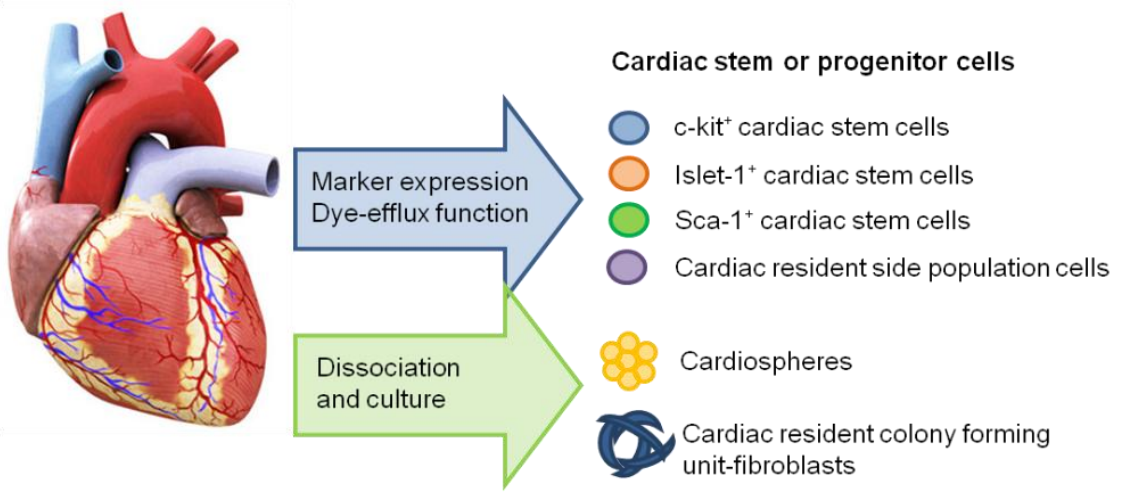

Figure 4. Cardiac progenitor cells residing in the adult heart. Cardiac progenitors cells identified based on the cell surface marker expression (e.g. c-Kit, Sca-1), genetic marker expression of transcription factors Islet-1 and functional properties such as dye-efflux function (cardiac side population cells), cardiosphere formation (cardiosphere cells or cardiosphere derived cells) and colony formation (colony forming unit-fibroblasts). Schematic adapted from Kikuchi et al. (2012).

Following this first study demonstrating the presence of endogenous progenitors in the adult heart with the capacity to differentiate into cardiac cells in vitro and in vivo, c-kit ${ }^{+}$CPCs (Bearzi et al. 2007, Miyamoto et al. 2010, Tallini et al. 2009, Zaruba et al. 2010) as well as other cardiac progenitors were isolated by several groups based on the expression of different stem cell-associated surface markers such as Sca-1 (Noack et al. 2012, Oh et al. 2003, Uchida et al. 2013, Wang et al. 2006), platelet derived growth factor receptor, alpha polypeptide (PDGFRa) (Chong et al. 2013, Noseda et al. 2015) or functional characteristics (e.g. cardiosphere formation, efflux of DNA binding dye and colony forming unit-fibroblasts) (Davis et al. 2009, Pfister et al. 2005) or origin (e.g. epicardium) (Limana et al. 2007) (Figure 4). However, it is still ambiguous whether these identified CPCs represent subsets of a common stem cell source in the heart with a transiently different phenotype or distinguishable stem cell types. The list of some of the defined CPCs so far is represented with their phenotypic characterization in Table 1. 


\begin{tabular}{|c|c|c|c|c|c|c|c|}
\hline & \multicolumn{3}{|c|}{$\begin{array}{c}\text { Stem cell markers } \\
\text { (c-kit/Sca-1/PDGFRa) }\end{array}$} & $\begin{array}{c}\text { Mesenchymal } \\
\text { markers }\end{array}$ & $\begin{array}{c}\text { Vascular cell } \\
\text { markers }\end{array}$ & $\begin{array}{c}\text { Hematopoietic } \\
\text { markers }\end{array}$ & References \\
\hline \multirow{7}{*}{$\begin{array}{l}\text { Surface marker } \\
\text { selected CPCs }\end{array}$} & c-kit+ & & & & & $\begin{array}{l}\text { CD34-, CD45-, } \\
\text { Lin- }\end{array}$ & (Beltrami et al. 2003) \\
\hline & c-kit+ & Sca-1+ & & & & $\begin{array}{l}\text { CD34+, } \\
\text { CD45+ } \\
\end{array}$ & (Matsuura et al. 2004) \\
\hline & & Sca-1+ & & & Flk-1-, CD31+ & CD34-, CD45- & (Oh et al. 2003) \\
\hline & c-kit- & Sca-1+ & & $\begin{array}{l}\text { CD90+, CD105+, } \\
\text { CD29+, CD44+, } \\
\text { CD73+ }\end{array}$ & CD31- & CD34- & (Tateishi et al. 2007) \\
\hline & & Sca-1+ & PDGFRa+ & $\begin{array}{l}\text { CD90+, CD105+, } \\
\text { CD29+, CD44+ }\end{array}$ & Flk-1-, CD31- & CD45- & (Chong et al. 2011) \\
\hline & c-kit- & Sca-1+ & & CD29+ & CD31- & CD34-, CD45- & (Takamiya et al. 2011) \\
\hline & c-kit- & Sca-1+ & PDGFR $\alpha+$ & $\begin{array}{l}\text { CD90-, CD105+, } \\
\text { CD29+, CD44+ }\end{array}$ & Flk-1-, CD31- & CD34-, CD45- & (Freire et al. 2014) \\
\hline \multirow{3}{*}{$\begin{array}{l}\text { Side population } \\
\text { CPCs }\end{array}$} & c-kit- & Sca-1+ & & CD44- & CD31-, Tie2+ & CD34-, CD45- & (Pfister et al. 2005) \\
\hline & c-kit+ & Sca-1+ & & CD29+, CD44+ & Flk-1+ & CD34+, CD45- & (Tomita et al. 2005) \\
\hline & c-kit+ & Sca-1+ & & & CD31- & $\begin{array}{l}\text { CD34+, } \\
\text { CD45+ } \\
\end{array}$ & (Martin et al. 2004) \\
\hline $\begin{array}{l}\text { Epicardial } \\
\text { progenitors }\end{array}$ & c-kit- & Sca-1+ & & & Flk-1-, CD31- & & (Smart et al. 2011) \\
\hline \multirow{2}{*}{$\begin{array}{l}\text { Cardiospheres/ } \\
\text { Mesoangioblast }\end{array}$} & c-kit+ & Sca-1+ & & CD29+ & Flk-1+, CD31+ & CD34+ & (Messina et al. 2004) \\
\hline & c-kit- & Sca-1+ & & & Flk-1-, CD31- & $\begin{array}{l}\text { CD34-, CD45-, } \\
\text { CD133- }\end{array}$ & (Ye et al. 2012) \\
\hline
\end{tabular}

Table 1. Putative CPCs and their phenotype in the adult heart.

\subsection{Cardiogenic potential of endogenous CPCs}

Utilizing direct genetic labeling strategies, based on the putative stem cell markers aforementioned (e.g. c-Kit and Sca-1), helped to clarify whether these markers only label stem or progenitor cells in the heart. Furthermore, it provided insight on cardiomyogenic potential of these endogenous CPCs through lineage tracing studies.

\subsubsection{C-Kit CPCs}

During embryogenesis in mice, c-Kit ${ }^{+}$cells appear as early as at embryonic day $(E)$ 6.5 in the cardiogenic mesoderm (Ferreira-Martins et al. 2012), endocardial cells at E8.5 and 9.5, and exhibit a broad distribution in the developing and adult heart including the inner layers of atrial and ventricular chambers with a preferential localization in the endothelium of vessels as well as subepicardium region (Bernex et 
al. 1996, Limana et al. 2007, Sultana et al. 2015, van Berlo et al. 2014). Pulse-chase labeling of $\mathrm{c}^{-\mathrm{Kit}^{+}}$cells during embryonic development in a tamoxifen inducible MerCreMer mouse model demonstrated the contribution of $\mathrm{c}-\mathrm{Kit}^{+}$cells to the different compartments of the adult heart (Figure 5) (Sultana et al. 2015, van Berlo et al. 2014). In contrast to other studies, suggesting the existence of c-Kit as CPCs in the myocardial interstitium (Bearzi et al. 2007, Beltrami et al. 2003, Urbanek et al. 2003), evidence for a broad distribution of $\mathrm{c}-\mathrm{Kit}^{+}$cells was demonstrated (Figure 5B).

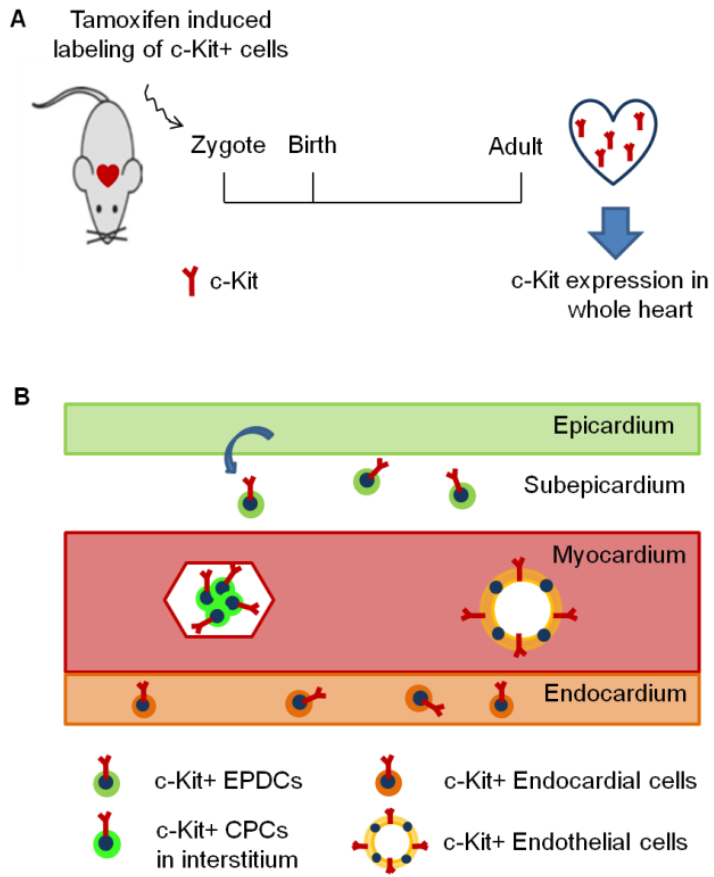

Figure 5. c-Kit cell progeny in the adult heart. A Lineage tracing of $\mathrm{c}-\mathrm{Kit}^{+}$cells in the developing heart in transgenic MerCreMer mice. B Spatial distribution of ${\mathrm{C}-\mathrm{Kit}^{+}}^{+}$cells in the adult heart. EPDC: Epicardium-derived progenitor cells.

Embryonic c-Kit ${ }^{+}$cells appeared to have contributed mainly to blood vessel associated endothelial cells in the adult heart $\left(\sim 80 \%\right.$ of $\mathrm{C}^{-\mathrm{Kit}^{+}}$cells co-labeled with $\left.\mathrm{CD} 31^{+}\right)$with rare differentiation into cardiomyocytes $(<0.1 \%$ of total cardiomyocytes) (Sultana et al. 2015, van Berlo et al. 2014). This limited cardiomyogenic potential of C-Kit ${ }^{+}$CPCs was also supported in the aging heart and under pathological conditions using the same transgenic mouse model and postnatal reporter activation (Sultana et al. 2015, van Berlo et al. 2014). 
A 3-fold increase in cardiomyogenic activity of $\mathrm{C}^{-K_{i t}{ }^{+}}$CPCs was reported after infarction; however, the amount of newly generated cardiomyocytes by $\mathrm{C}-\mathrm{Kit}^{+} \mathrm{CPCs}$ remained too low for a palpable endogenous cardioregenative response (van Berlo et al. 2014). Conversely, Ellison et al. proposed that $\mathrm{C}-\mathrm{Kit}^{+} \mathrm{CPCs}$ are indispensable for cardiac regeneration (Ellison et al. 2013). Using lentiviral labeling of endogenous c$\mathrm{Kit}^{+} \mathrm{CPCs}$, they demonstrated that $10 \%$ of the newly formed myocytes in the injury site after myocardial infarction stemmed from $\mathrm{C}^{-\mathrm{Kit}^{+}} \mathrm{CPCs}$. These CPC-derived cardiomyocytes appeared functional and contained sarcomeric structures, but in immature state (Ellison et al. 2013).

\subsubsection{Sca-1 CPCs}

Sca- $1^{+}$CPCs were first identified by Matsuura et al. reporting that Sca- $1^{+}$cells isolated from the adult mouse heart were able to differentiate into beating cardiomyocytes in vitro (Matsuura et al. 2004). Moreover, Sca- $1^{+}$CPCs had the ability to differentiate into adipocytes and osteocytes, showing MSC-like characteristics. Although a human Sca-1 epitope has not been identified so far, antibody selection for mouse Sca-1 was applied successfully to isolate Sca-1+-like progenitors from the human heart (Smits et al. 2009). Recent studies in the mouse demonstrated that the majority of Sca- $1^{+}$cells in the heart were endothelial cells $(>70 \%)$ and included a small fraction of smooth muscle cells $(<5 \%)$ (Uchida et al. 2013). Additional Sca- $1^{+}$cells in the myocardial interstitium were most likely comprised of cardiac fibroblasts (Furtado et al. 2014) and Sca- $1^{+}$progenitors closely associated with the cardiomyocyte basal lamina, expressing stem cell surface markers: c-Kit, CD34 and Abcg2 (ATP-binding cassette, sub-family G, member 2) (Uchida et al. 2013). In other studies, Sca- $1^{+}$CPCs that share some of the stem/progenitor cell markers (c-Kit, CD34) have been found in epicardial progenitor cells in the adult epicardium (Limana et al. 2007). In addition, Sca- $1^{+}$CPCs have been found to express the epicardium-derived progenitor cell marker, PDGFRa (Chong et al. 2011). Together, these findings suggest a epicardial origin of Sca- $1^{+}$ cells.

Given the specificity of PDGFRa as a marker for epicardial progenitors (Chong et al. 2011. Chong et al. 2013) and the role of epicardium in the formation of 
cardiovascular compartment and stromal cells in the developing heart, Sca$1^{+} / \mathrm{PDGFRa}^{+}$CPCs were suggested to have the potential to differentiate into interstitial and smooth muscle cells, but not endothelial cells (Chong et al. 2011). This finding was contrary to recent findings, where most of the Sca-1+ CPCs were shown to differentiate into endothelial cells during physiological development (Uchida et al. 2013). This contradiction regarding the differentiation potential of Sca- $1^{+}$cells might be explained either with the existence of two subtypes of Sca- $1^{+}$progenitors (Sca$1^{+} /$PDGFR $^{+}$and Sca-1+PDGFR), as demonstrated in the aorta (Cho et al. 2013), giving rise to different cell types in the heart or the heterogeneity of Sca- $1^{+}$cells, composed of mainly endothelial cells which could mask the fate tracking of actual of Sca- $1^{+}$progenitors in the heart (Uchida et al. 2013). Nevertheless, with respect to cardiomyogenic potential of Sca- $1^{+}$CPCs, lineage tracing of Sca- $1^{+}$cells continuously labeled from embryonic stage to postnatal development revealed that Sca- $1^{+}$cells were able to contribute to cardiomyocyte renewal continuously under normal aging, but in a very low amount suggesting for their limited cardiomyogenic potential. Furthermore, they did not actively contribute to new cardiomyocyte formation even after injury (Uchida et al. 2013).

\subsubsection{Vessel resident progenitor cells}

So far, different types of blood vessel associated progenitors were identified based on their localization and specific marker expression, such as pericytes, mature vessel medial and adventitial progenitor cells (Kovacic and Boehm 2009). Pericytes have been discussed as mesenchymal stem cell (MSC)-like cells in the heart (Figure 6), with similar surface marker expression profile (e.g. Sca-1), the ability to undergo trilineage differentiation into osteoblasts, chondrocytes, and adipoyctes (Crisan et al. 2008) and the competency for self-renewal (Sacchetti et al. 2007, Wong et al. 2015). 


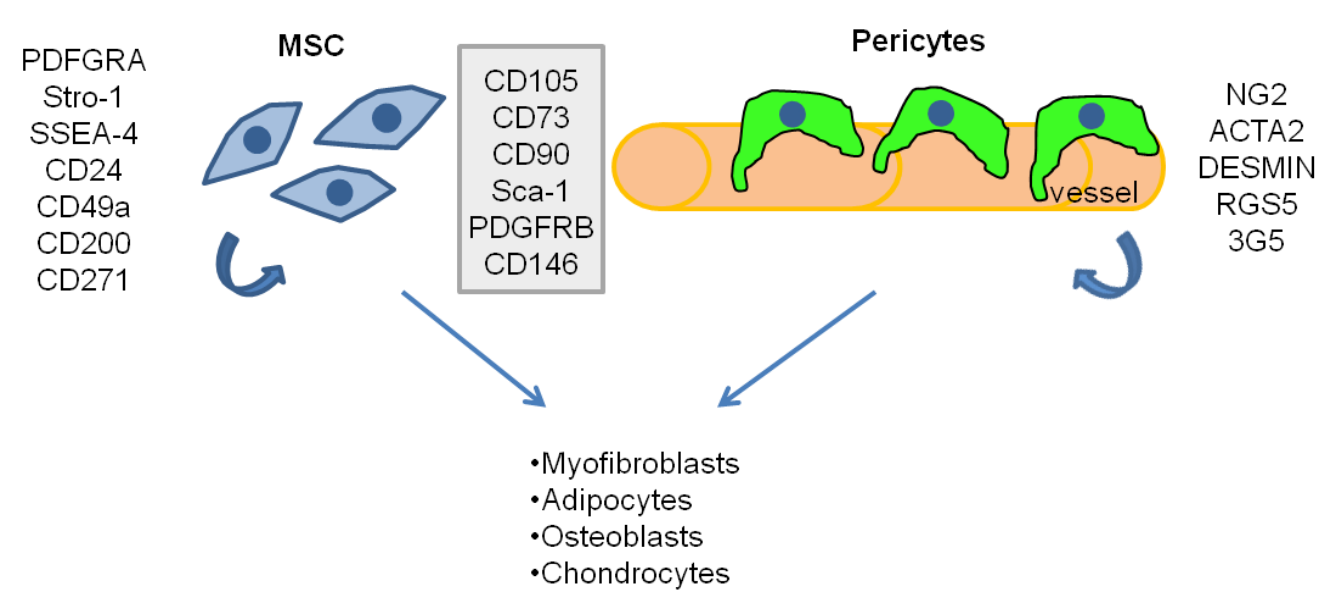

Figure 6. Unique and shared properties of pericytes and mesenchymal stem cells. Schematic adapted from Wong et al. (2015).

Pericytes have been recently defined as progenitors for smooth muscle cells, arising from epicardium during embryonic heart development. In addition, pericytes that are clonally related to smooth muscle cell are able to remain around the coronary arteries or tunica adventitia as cardiac progenitors, if they do not develop into smooth muscle cell (Volz et al. 2015). Consistent with this, the possibility that there are progenitor cells localizing in the tunica media or adventitia of mature vessels was suggested by several studies. Progenitor cells expressing stem cell markers including c-Kit, Sca-1, CD34 and residing in the tunica adventitia with the potential to differentiate into smooth muscle cells were recently identified (Hu et al. 2004). Another study reported that there are also progenitor cells, so called side population cells located in the tunica media that express Sca-1 and CD34, being capable of giving rise to smooth muscle and endothelial cells (Sainz et al. 2006). In addition to pericytes and adventitial cells, Kramann et al. reported another type of perivascular progenitors residing in pericyte niche (Kramann et al. 2015), GLI family zinc finger 1 (Gli1) ${ }^{+}$cells around the vasculature in close proximity to pericytes. These pericyte nicheassociated cells do not show pericyte-specific markers such as CD146 and NG2 (CSPG4; chondroitin sulfate proteoglycan 4). Additionally, these cells display MSC properties and markers including Sca-1 and PDGFRa, suggesting a epicardiumderived progenitors origin (Smith et al. 2011). Given the fact that virtually all the organs contain MSCs with subendothelial localization, it is likely that vasculature in the heart serve as a niche that hosts a number of MSC-like stem or progenitor cells 
(Kovacic and Boehm 2009, Wong et al. 2015). However, due to the limited understanding on the origin of cardiac progenitor cells and the heterogeneous expression profile of stem cell surface markers (e.g. c-Kit and Sca-1) in the heart, it is still not clear if these progenitors simply originate from the vasculature or are descendants of a common stem cell source (e.g. epicardium) or remnants of cardiovascular progenitors from embryonic development (Valente et al. 2014). The spatial distribution of putative cardiac stem/progenitor cells in the heart is schematically displayed in Figure 7; the relationship between these progenitors still remains to be defined.

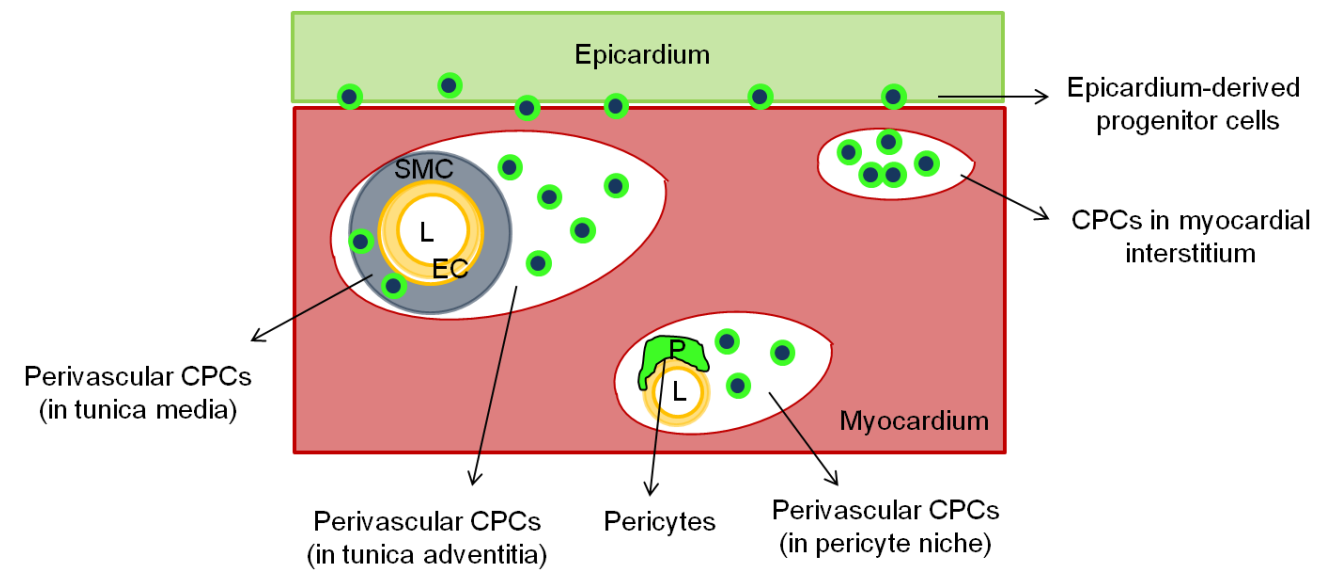

EC: Endothelial cell, L: Lumen, P: Pericye, SMC: Smooth muscle cell

Figure 7. Schematic overview of the spatial distribution of putative CPCs.

\subsection{Exogenous regeneration by CPCs}

Endogenous cardiac repair by resident CPCs is limited in the adult heart. Thus, implantation of CPCs into the myocardium or their pharmacological activation has been proposed as therapeutic strategies in heart disease (Beltrami et al. 2003). Accordingly, small and large animal models (e.g. rat and pig) were utilized to assess the cardio-regenerative potential of $\mathrm{c}-\mathrm{Kit}^{+} \mathrm{CPCs}$ and also to develop strategies to enhance their retention rate upon delivery into the myocardium. In vitro expanded and growth factor (e.g. HGF and IGF-1) stimulated c-Kit ${ }^{+}$CPCs displayed robust engraftment and survival within 2 days after intramyocardial injection and gave rise to 
vascularized myocardium, attenuating left ventricular dysfunction after myocardial infarction (Tillmanns et al. 2008). Similarly, autologous c-Kit ${ }^{+}$CPC implants induced functional myocardial tissue regeneration within chronically scarred myocardium in rat and pig heart (Bolli et al. 2013, Rota et al. 2008, Tang et al. 2010).

Although the potential mechanisms underlying the cardio-supportive effects of $\mathrm{c}-\mathrm{Kit}^{+}$ CPCs still needs to be defined, it is unlikely that differentiation of transplanted CPCs can explain the observed improvements. Instead, paracrine mediated effects initiating endogenous repair through recruitment of endogenous CPCs are discussed as alternative mechanism. Collectively, available animal studies provided convincing evidence for feasibility and safety of $\mathrm{c}-\mathrm{Kit}^{+} \mathrm{CPC}$ implantation with additional hints for efficacy (van der Spoel et al. 2011).

\subsection{Cardiac stem cell based therapy}

Various cell types from different sources have been tested experimentally and clinically for their capacity to regenerate the heart using different routes of administration into the myocardium. Cell sources used in direct intracoronary or intramyocardial transplantations include; autologous bone marrow (BM)-derived cells (unselected BM-derived mononuclear and-mesencyhmal stem cells) (Bartunek et al. 2013, Lunde et al. 2006, Meyer et al. 2009, Mushtaq et al. 2014, Perin et al. 2012, Roncalli et al. 2011), skeletal muscle progenitors (satellite cells) (Menasche et al. 2008), peripheral blood cells (Assmus et al. 2007), adipose tissue-derived MSCs (Houtgraaf et al. 2012), heart-derived stem/progenitor cells (c-Kit ${ }^{+}$and cardiosphere progenitors) (Bolli et al. 2011, Chugh et al. 2012, Makkar et al. 2012). Besides direct intramyocardial delivery of exogenous cells, mobilization of endogenous progenitors from bone marrow by systemic administration of defined cytokines (e.g. erytropoieitn [EPO] and granulocyte-colony stimulating factor [G-CSF]) were also evaluated as alternative strategies (Achilli et al. 2010, Taniguchi et al. 2010). Candidate cell populations to induce myocardial regeneration and their delivery routes to the heart are summarized in Figure 8. 


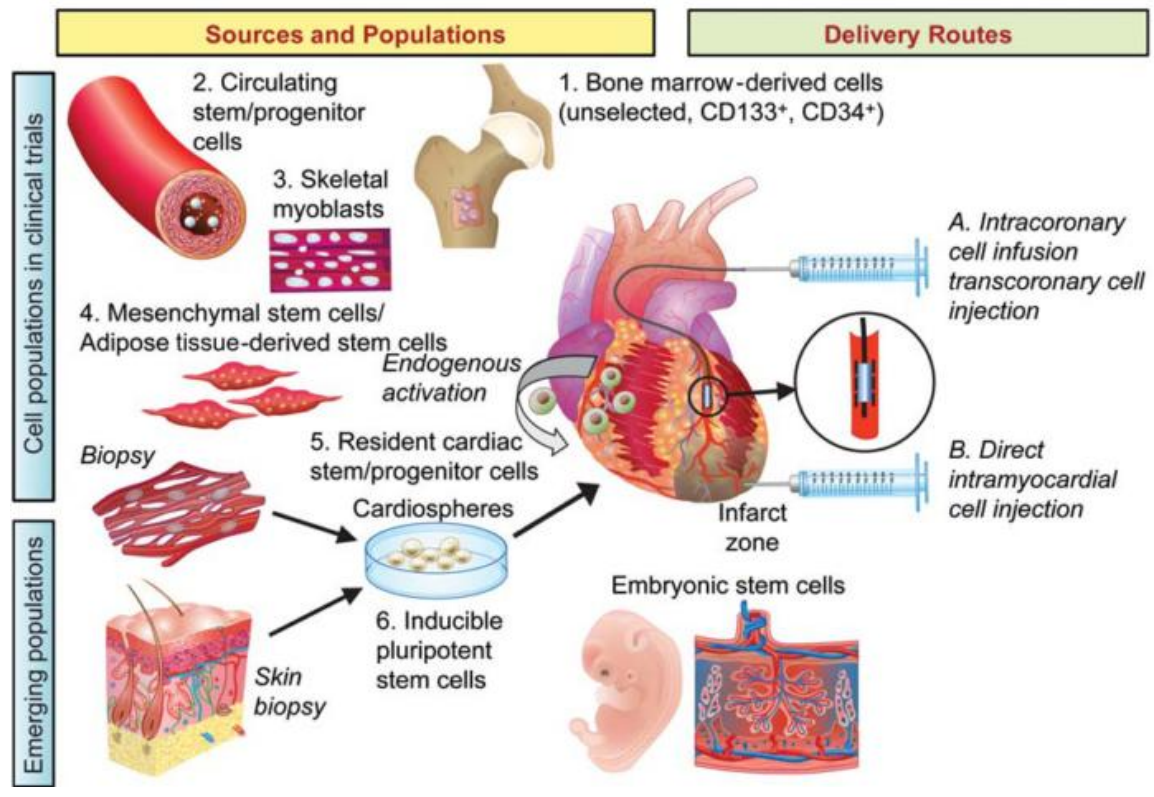

Figure 8. Clinically tested cell sources for heart regeneration. Schematic adapted from Doppler et al. (2013).

Being easy to harvest and apparently immune privileged, BM-derived stem/progenitor cells have been considered feasible and safe to be applied in clinical therapies without adverse effects so far, although it is also speculated that MSCs can transform into malignant tumors (Miura et al. 2006, Rubio et al. 2005). Unselected BM-derived mononuclear cells containing a mixture of endothelial progenitors, angioblasts and hematopoietic stem cells were thought to be an ideal cell source to induce neovascularization and new cardiomyocyte formation (Pavo et al. 2014). These cells, however, displayed a low abundance of relevant progenitors, modest clinical results and lack of convincing evidence for hematopoietic stem cells to differentiate into cardiomyocytes. Hence, the mode of action remains, despite the in some studies clinically observed beneficial effects and a suggested paracrine activity, elusive (Suzuki 2015).

The discovery of the heart containing endogenous cardiac progenitors with the capability to differentiate into vascular cells and cardiomyocytes initiated the isolation and in vitro expansion of these cells to be tested in several Phase Ila/b human clinical trials. These include the (1) cardiosphere-derived autologous stem cells to reverse ventricular dysfunction (CADUCEUS; (Makkar et al. 2012)), (2) cardiac stem 
cells in patients with ischemic cardiomyopathy (SCIPIO; (Bolli et al. 2011)) and (3) autologous human cardiac-derived stem cell to treat ischemic cardiomyopathy (ALCADIA; (Takehara et al. 2012)) trials. In addition, another clinical trial, so called safety and efficacy evaluation of intracoronary infusion of allogeneic human cardiac stem cells in patients with acute myocardial infarction (CARE-MI) is ongoing in Phase I and II under the sponsorship of Coretherapix company, Spain (CARE-MI trial; Clinical Trial Identifier: NCT02439398).

In the CADUCEUS trial, patients were treated with 25 million autologous cardiosphere-derived progenitor cells (CDCs) isolated from right ventricular endomyocardial biopsies and injected through intracoronary infusion into the infarct related artery 1.5-3 months after myocardial infarction. The hypothesis was that scar tissue would be converted into viable myocardium (Makkar et al. 2012). Injection of CDCs significantly reduced the infarct size, increased the amount of viable myocardium as well as thickness in the peri-infarct zone at 6-12 months follow up, although there was no difference detected in left ventricular (LV) ejection fraction (Makkar et al. 2012). The SCIPIO trial was performed in patients with heart failure due to ischemic cardiomyopathy injecting $0.5-1$ million of autologous $\mathrm{c}-\mathrm{Kit}^{+} \mathrm{CPCs}$ derived from the atrial appendage. A reduction in infarct size and improvement in ejection fraction were reported after one and four year follow-up (Bolli et al. 2011). The ongoing ALCADIA trial differs from these previous studies in that stem cell delivery is combined with a biodegradable gelatin hydrogel scaffold for sustained FGF-2 release and enhanced cell retention (ALCADIA trial; Clinical Trial Identifier: NCT00981006). Taken together, these first clinical trials revealed that autologous transplantation of CPCs is feasible and safe.

More recently and as a consequence of the failure of autografts to form new cardiomyocytes there is a paradigm shift from autologous to allogenic implantations (CARE-MI trial; Clinical Trial Identifier: NCT02439398). Lauden et al. showed that allogeneic c-Kit-selected human CPCs (hCPCs) might exhibit a beneficial immunomodulatory effect. hCPCs express programmed death ligand 1 (PD-L1) protein which plays an important role in immunesuppression via acting as an inhibitory signal on the proliferation and activation of $\mathrm{CD} 8^{+} \mathrm{T}$-cells and activating regulatory T-cells. In addition, the immunomodulatory capacity of hCPCs was not 
altered upon treatment with interferon gamma (IFN- $\mathrm{Y}$ ), which is a pro-inflammatory cytokine secreted during myocardial infarction and infarct remodelling. This phenotype of hCPCs and the possibility for cryopreservation suggest that cell banks with allogeneic c-Kit-selected hCPCs may find an application in patients with acute myocardial infarction (Lauden et al. 2013).

Although there is first evidence of clinical efficacy, the main mechanisms for these cardio-supportive effects of transplanted $\mathrm{c}-\mathrm{Kit}^{+} \mathrm{CPCs}$ are not yet fully understood. There are, however, several mechanisms proposed for these beneficial effects (Lauden et al. 2013), that can be summarized as direct and indirect effects of exogenously transplanted CPCs on the maintenance of heart structure and function. CPCs delivered into the infarcted myocardium may directly contribute to cardiomyogeneis and angiogenesis either via paracrine signaling (e.g. growth factors and cytokines) enhancing proliferation and survival of endogenous cardiomyocytes and vascular cells or giving rise to new cardiomyocytes and vascular cells (smooth muscle cells and endothelial cells). Besides this direct effect of CPCs on cardiac regeneration, they may also indirectly mediate cardio-supporting activity through regulating heart injury responses (e.g. reduced inflammation, fibrosis and remodeling) and activating endogenous CPCs to differentiate into cardiac cells. All these mechanisms are schematically summarized in Figure 9. 


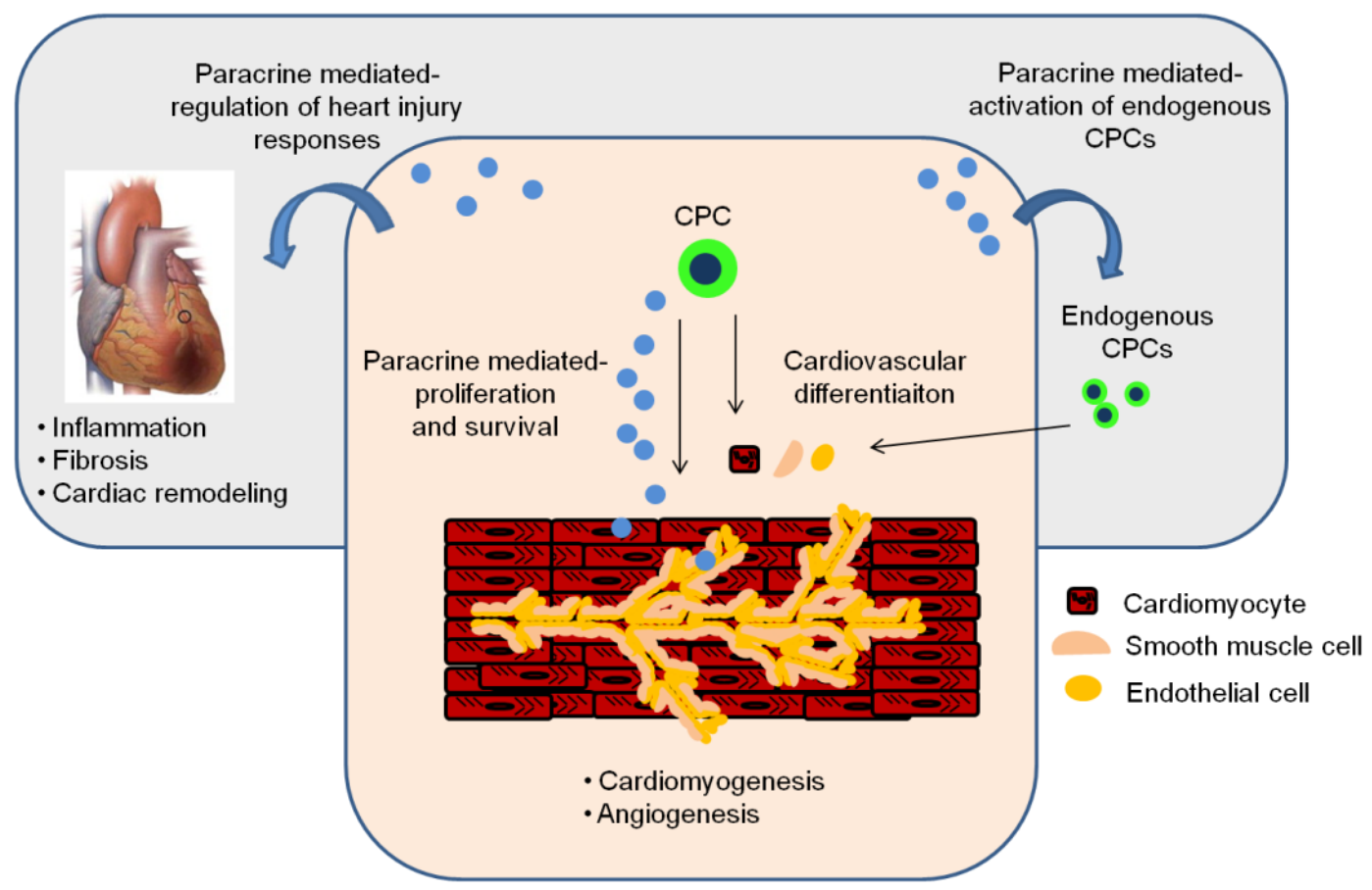

Figure 9. Schematic overview of potential mechanisms involved in heart repair upon CPC transplantation in vivo. Core panel (pink-colored): Direct contribution of CPCs to heart regeneration either by direct transdifferentiation or paracrine signaling. Outer panel (grey-colored): Indirect contribution of CPCs to heart regeneration and function through activating endogenous CPCs and regulating heart injury responses (e.g. reduced inflammation, fibrosis and remodeling).

\subsection{EHM as an in vitro cardiac muscle model}

Cardiac tissue engineering focuses on the development of biomimetic artificial cardiac muscle constructs. Engineered heart muscle (EHM) shows structural and physiological characteristics of native heart muscle, which renders it a high-fidelity in vitro platform to also study cell-cell interactions within a three-dimensional heart muscle context (Naito et al. 2006, Tiburcy et al. 2011, Zimmermann et al. 2002). Characteristic properties of EHM comprise: 1) the formation of a functional syncytium; 2) terminal differentiation of cardiomyocytes; 3) organotypic maturation with structural and functional properties of the postnatal heart (Christalla et al. 2012). Stromal cells play an essential role in this process by providing and maintaining a cardio-instructive extracellular matrix (ECM) milieu and paracrine support to guide heart muscle development in vitro (Naito et al. 2006, Tiburcy et al. 2011). 


\subsection{Aims of the study}

A better understanding of the biological activity of CPCs may help to refine therapeutic strategies aiming at the regeneration of the failing heart. We hypothesized that EHM could be used as an in vitro heart surrogate platform to scrutinize and define the cardio-supportive effects of CPCs.

This study tested the following specific hypotheses:

1) CPCs support functional heart muscle formation in vitro.

2) CPCs elicit cardioprotective effects upon hypoxic injury.

The hypotheses were investigated in EHM from rat, mouse, and human cells as indicated. A new transgenic reporter model was established to study the role of hypoxia. 


\section{Materials and Methods}

\subsection{Preparation of cardiomyocytes}

Cardiomyocytes were harvested from neonatal rat heart (section 2.1.1) as well as mouse (section 2.1.2) and human (section 2.1.3) embryonic stem cells. Organ harvest was approved by the responsible animal protection authorities (LAVES - AZ: 10.13/10.14). The use of human embryonic stem cells was approved by the RobertKoch-Institute (AZ: 1710-79-1-4-16) according to §6 Stammzellgesetz (StZG).

\subsubsection{Neonatal rat cardiomyocytes}

Neonatal rat hearts (day 0-3) were dissociated by using a digestion protocol based on trypsin/DNAse I (Zimmermann et al. 2000). Cells were thereafter pre-plated for 1 hour at $37{ }^{\circ} \mathrm{C}, 5 \% \mathrm{CO}_{2}$ on plasma treated cell culture dishes. The non-attached cell suspensions were harvested as myocyte fraction and designated as neonatal rat cardiomyocytes (NRCMs). The purity of cardiomyocytes was determined by flow cytometry (BD LSR II; BD Biosciences) of cells stained for $\alpha$-sarcomeric actinin (Sigma-Aldrich, see Appendix A3 for antibody dilution and section 2.6.2 for immunostaining protocol).

\subsubsection{Mouse embryonic stem cell-derived cardiomyocytes}

Upon mating of transgenic ROSA26 ODD-Luc/+ (heterozygous for ODD-Luc knock-in in the ROSA26 locus; see Appendix A4 for detailed background of the mice strain), zygotes at blastocyst stage were harvested and cultured on inactivated mouse embryonic fibroblasts (mEFs). Inner cell mass outgrowths from blastocysts were isolated and cultured on mEFs to give rise to ODD-Luc mouse embryonic stem cell (mESC) colonies. Subsequently, ODD-Luc mESCs were electroporated with a DNA construct expressing a neomycin resistance gene (neoR) under the transcriptional control of cardiomyocyte restricted alpha-myosin heavy chain ( $\mathrm{aMHC}$ ) promoter and a hygromycin resistance gene (hygroR) under ubiquitiously active phosphoglycerate (PGK) promoter (Klug et al. 1996) and selected under hygromycin $(500 \mu \mathrm{g} / \mathrm{ml}$ for 7 days). The establishment of bitransgenic ODD-Luc $x$ aMHC-neoR mESC line was 
performed by Andreas Schraut (Institute of Pharmacology and Toxicology, University Medical Center Göttingen). ODD-Luc x aMHC-neoR mESCs were expanded on feeder layers composed of mitotically arrested MEFs ( $\mathrm{y}$-irradiated with $30 \mathrm{~Gy}$ in Biobeam 8000, STS GmbH) cultivated in mESC culture medium (Appendix A1).

Cardiac differentiation was performed in $100 \mathrm{ml}$ spinner flask cultures $\left(0.1 \times 10^{6} / \mathrm{ml}\right.$ seeding density) for 11 days, followed by additional 7 days of cardiomyocyte selection with G418 $(400 \mu \mathrm{g} / \mathrm{ml})$ in mESC differentiation medium (Appendix A1) as shown in Figure 10. Spontaneously beating cardiac bodies (CBs) were dissociated with a digestion protocol based on collagenase type I solution (Appendix A1) and trypsin-EDTA $(0.25 \%)$. The purity of cardiomyocytes was detected by flow cytometry analysis of sarcomeric a-actinin staining (see Appendix A3 for antibody dilution and section 2.6.2 for immunostaining protocol). Cardiomyocyte yield per input mESCs was in the range between 1:1 and 1:2 after spontaneous differentiation and selection. Purity of cardiomyocytes is represented as a result in section 3.2.1.

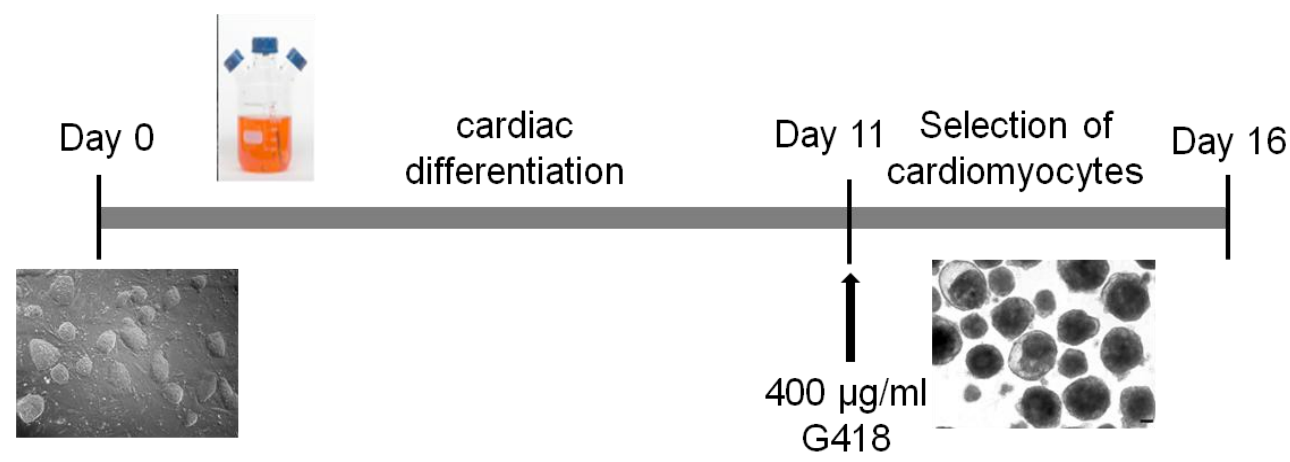

Figure 10. Cardiac differentiation of mESCs. Schematic representation of mouse cardiac differentiation: suspension culture of mESCs cultivated in spinner flasks for 11 days to induce spontaneous cardiac differentiation, followed by 7 days of selection of spontaneously beating cardiac bodies (CBs) with the addition of G418 $(400 \mu \mathrm{g} / \mathrm{ml})$. 


\subsubsection{Human embryonic stem cell-derived cardiomyocytes}

The human embryonic stem cell line "HES2" (Embryonic Stem Cell International, Singapore), genetically modified to stably and ubiquitously express a tandem dimer red fluoresence protein (tdRFP) from the ROSA26 locus (finally designated hES2.R26) was kindly provided by Gordon Keller (Irion et al. 2007). hES2-RFP cells were maintained and differentiated under serum free conditios (Hudson et al. 2012) with minor modifications. Briefly, hESCs were initially cultured on irradiated hFFs in hESC culture medium (Appendix A1) and passaged using EDTA digestion solution (0.5 mol/L, pH 8, AppliChem) for expansion and single cell adaptation. Thereafter, hES2.R26 cells were plated on feeder free, Matrigel ${ }^{\mathrm{TM}}$ (Corning, 1:30 diluted in $1 \mathrm{x}$ PBS)-coated flasks in hESC-conditioned medium (Appendix A1) with every day medium change. Upon plating of $\mathrm{hESC}$ on Matrigel-coated plates with a seeding density of $5 \times 10^{4}-1 \times 10^{5}$ cells $/ \mathrm{cm}^{2}$ in hESC-conditioned medium for one day, hESCs were rinsed with hCM medium (Appendix A1) and cultured in mesoderm-induction medium (Appendix A1) for 3 days. After another washing step with hCM medium, cells were cultured in cardiac specification medium (Appendix A1) for the following 10 days. After two weeks of differentiation, cardiomyocytes were metabolically selected (Tohyama et al. 2013) in hCM selection medium (Appendix A1) for 5 days to obtain a highly enriched cardiomyocyte population.

Following the purification step, cardiomyocytes were washed two times with 1x PBS at room temperature (RoT) and subsequently, incubated in hCM digestion solution (Appendix A1) for 4 minutes at RoT and 10 minutes at $37^{\circ} \mathrm{C}$ for digestion. Harvested cardiomyocytes were either seeded on cell culture flasks pre-coated with Matrigel ${ }^{\mathrm{TM}}$ (Corning, 1:30 diluted in 1x PBS) or directly used to generate human engineered heart muscle (hEHM). The purity of cardiomyocytes was detected by flow cytometry analysis of sarcomeric $\alpha$-actinin staining (see Appendix A3 for antibody dilution and section 2.6.2 for immunostaining protocol). 


\subsection{Preparation of non-myocytes}

Cardiac progenitor cells (CPCs) from mouse (mCPC; section 2.2.1) and human ( $\mathrm{hCPC}$; section 2.2.2) heart were used. Fibroblasts were isolated from mouse embryos (MEF; section 2.2.3) and human foreskin (hFF; section 2.2.4). Human cardac fibroblasts (hCF) were acquired from Lonza.

\subsubsection{Mouse CPCs}

We used two different types of murine cardiac progenitor cells (mCPCs); Sca-1CPCs and c-Kit-CPCs isolated from adult mouse heart. GFP-labeled Sca-1-mCPCs overexpressing micro RNA 133a (miR-133a-CPCs) and micro RNA control (miRcontrol-CPCs) were kindly provided by Antonio Bernad (Izarra et al. 2014). GFPlabeled c-Kit-mCPCs (GFP ${ }^{+}$mCPCs) were kindly provided by Mark Sussman (Fischer et al. 2009). CPCs were maintained in mCPC medium (Appendix A1) under $21 \% \mathrm{O}_{2}$ and passaged with $0.05 \%$ Trypsin-EDTA (phenol red, \#25300-054, Gibco) at $80 \%$ confluency.

\subsubsection{Human CPCs}

Human CPCs (hCPCs) isolated based on the c-Kit surface protein from right atrial appendages of patients were kindly provided by Coretherapix Biomedicine, Spain (Lauden et al. 2013). hCPCs (wild type or GFP-labeled; see Appendix A4) were delivered in frozen aliquots and directly used after thawing to generate hEHM without being cultured in our laboratory.

\subsubsection{Mouse embryonic fibroblasts}

Mouse embryonic fibroblasts (mEFs) were harvested at days post coitum (d.p.c) 12.5-13.5 by Andreas Schraut. mEFs were maintained in mEF medium (Appendix A1). For passaging, mEFs were enzymatically digested with $0.25 \%$ Trypsin-EDTA (\#25200056, Gibco) at 80\% confluency and split at a 1:3 ratio for further expansion. 


\subsubsection{Human foreskin fibroblasts}

Human foreskin fibroblasts (hFFs) were purchased from the American Type Culture Collection (ATCC). hFFs were passaged with TrypLE (Tryple ${ }^{\mathrm{TM}}$ Express Enzyme 1X, no phenol red, \#12604-013, Gibco) and cultured in hFF medium (Appendix A1). hFFs were lentivirally transduced to generate a stable cell line expressing GFP. Lentiviral particles were generated by co-transfecting pGIPZ with pMD26 and PSPAX2 helper plasmids into human embryonic kidney (Sagoo et al.) 293-T cells using the FuGENE HD transfection protocol (Promega). After a medium change, virus-containing medium was harvested every 24 hours for 72 hours. Viral particles were pooled and concentrated by centrifugation using Amicon ${ }^{\circledR}$ Ultra Centrifugal Filters (Millipore). Concentrated virus was stored at $-80^{\circ} \mathrm{C}$. To transduce $\mathrm{hFFs}$, cells were treated with polybrene $(10 \mu \mathrm{g} / \mathrm{ml})$ in culture medium containing $0.5 \%$ fetal bovine serum (FBS; \#10270, Gibco) at $37{ }^{\circ} \mathrm{C}$ for 10 minutes. Thereafter, virus containing medium was added drop wise on top of the cells and incubated at $5 \% \mathrm{CO}_{2}, 37^{\circ} \mathrm{C}$ for 24 hours. Finally, the medium was exchanged with fresh hFF medium and transduced hFFs were selected with $1 \mu \mathrm{g} / \mathrm{ml}$ puromycin for 5 days.

\subsection{EHM}

Engineered heart muscle (EHM) was generated from neonatal rat heart and mouse or human embryonic stem cell-derived cardiomyocytes with CPCs or fibroblasts as indicated below.

\subsubsection{Rat EHM}

NRCMs were mixed with adult mouse heart-derived miR-control CPCs or miR-133aCPCs in a $70 / 30 \%$ ratio $\left(2.5 \times 10^{6}\right.$ cell/EHM) and mixed with a master mix containing collagen type I from rat tail according to the amounts mentioned in Table 2. Rat EHMs (rEHMs) were made as previously described (Godier-Furnemont et al. 2015, Zimmermann et al. 2000). Briefly, the rEHM reconstitution mixture was cast into circular molds (inner/outer diameter: 8/16 mm) (Naito et al. 2006) and supplemented with rEHM medium (Appendix A1) following incubation for 1 hour at $37^{\circ} \mathrm{C}$. rEHMs were allowed to condense for 7 days in the casting mold. Subsequently, rEHMs were 
transferred and maintained on phasic stretchers from culture day 7 to day 12 to induce functional maturation under mechanical loading (Figure 11).

\begin{tabular}{|l|c|}
\hline Number of rEHMs & 4x rEHMs \\
\hline & Volume $(\mu \mathrm{l})$ \\
\hline Rat collagen $(3.4 \mathrm{mg} / \mathrm{ml})$ & 940 \\
\hline $2 \times \mathrm{rEHM}$ DMEM (Appendix A1) & 940 \\
\hline $0.1 \mathrm{~N} \mathrm{NaOH}$ & 182 \\
\hline Matrigel $^{\mathrm{TM}}$ & 400 \\
\hline Cell suspension $\left(2.75 \times 10^{6} / \mathrm{EHM}\right)$ & 1738 \\
\hline Total volume & 4200 \\
\hline
\end{tabular}

Table 2. rEHM master mix.

Day 0 Day 7

Day 12

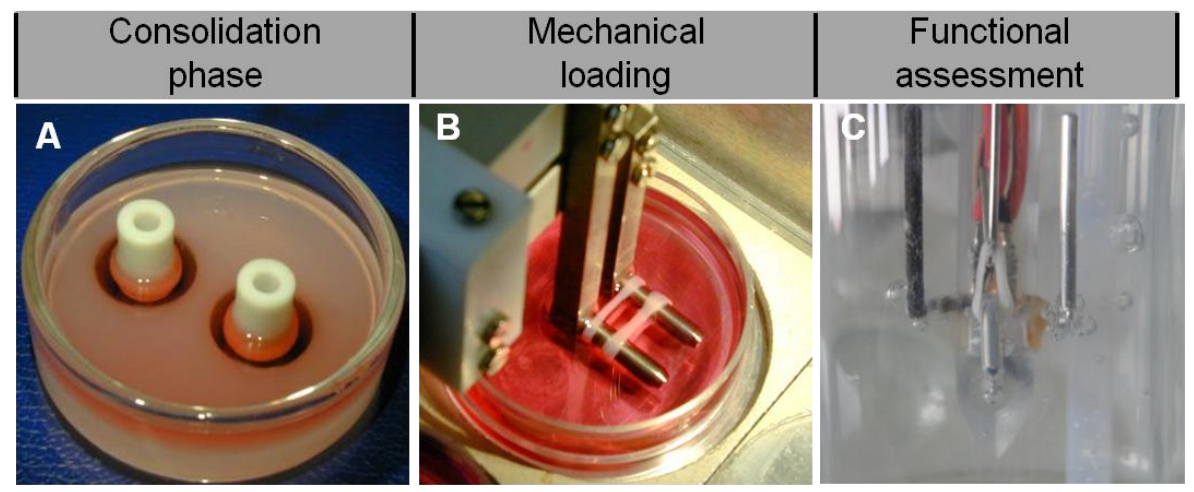

Figure 11. Construction of rat EHM. A Rat EHMs were maintained in casting molds for consolidation for 7 days. B Rat EHMs were exposed to mechanical loading on phasic stretchers at $1 \mathrm{~Hz}$ for 24 hours and $2 \mathrm{~Hz}$ for the following 4 days until force measurement (Zimmermann et al. 2002). C Measurement of force of contraction (FOC) under isometric conditions in a thermostatted organ bath $\left(37^{\circ} \mathrm{C}\right)$ filled with Tyrode's solution (Appendix A1).

\subsubsection{Mouse EHM}

mESC-derived cardiomyocytes (mCMs) were mixed with $\mathrm{GFP}^{+} \mathrm{mCPCs}$ or mEFs in a $70 / 30 \%$ ratio to generate mouse EHM (mEHM). Co-cultured cells were subsequently mixed with a master mix containing collagen type I isolated from rat tail (Table 3). Briefly, the mEHM cell mixture was cast in circular molds with an inner/outer diameter 
of 2/4 mm (Tiburcy et al. 2014) as shown in Figure 13 and supplemented with mEHM medium (Appendix A1) following incubation for 1 hour at $37^{\circ} \mathrm{C}$. On culture day 3 , mEHMs were transferred onto static stretchers where they were exposed to mechanical loading for an additional 11 days.

\begin{tabular}{|l|c|}
\hline Number of mEHMs & $4 \times$ mEHMs \\
\hline & Volume $(\mu \mathrm{l})$ \\
\hline Rat collagen $(4.2 \mathrm{mg} / \mathrm{ml})$ & 399 \\
\hline $2 \times \mathrm{mEHM}$ DMEM $($ Appendix $\mathrm{A} 1)$ & 399 \\
\hline $0.1 \mathrm{~N} \mathrm{NaOH}$ & 78.5 \\
\hline Cell suspension $\left(1.5 \times 10^{6} / \mathrm{EHM}\right)$ & 1223.5 \\
\hline Total volume & 2100 \\
\hline
\end{tabular}

Table 3. mEHM master mix.

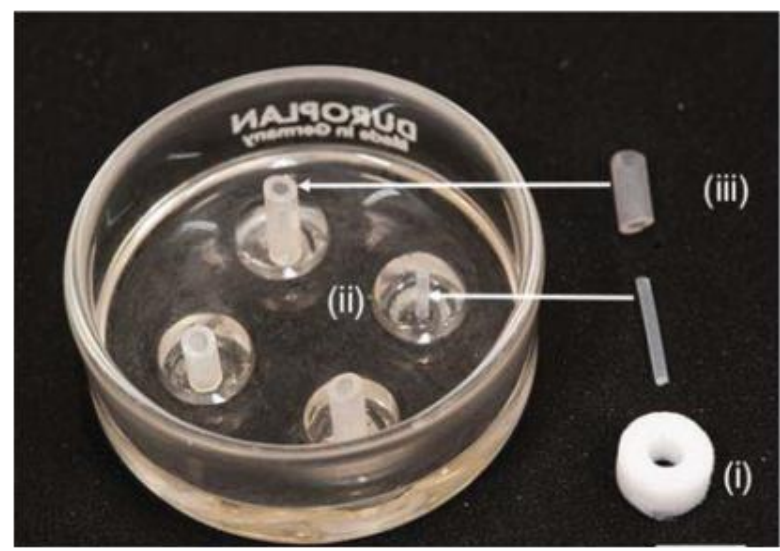

Figure 12. Casting mold for mouse and human EHM. Teflon disc (i) with $11 \mathrm{~mm}$ diameter, $5 \mathrm{~mm}$ height) was used as a spacer upon addition of silicone. Removal of the Teflon discs after hardening of the silicone established circular recesses (Tohyama et al. 2013). Silicone tubing (iii) with inner/outer diameter of 2/4 mm (Tiburcy et al. 2014) was placed on a central silicone core (Tohyama et al. 2013) in the center of recesses, providing the circular space for the casting of the EHM mixture (Soong et al. 2012). 


\subsubsection{Human EHM}

Human engineered heart muscle ( $\mathrm{hEHM}$ ) was generated by mixing $\mathrm{RFP}^{+} \mathrm{hCMs}$ with $\mathrm{GFP}^{+}$hCPCs or $\mathrm{GFP}^{+}$hFFs in $70 / 30 \%$ ratio in a master mix containing bovine collagen type I (Table 4).

\begin{tabular}{|l|c|}
\hline Number of hEHMs & $4 \times \mathrm{hEHMs}$ \\
\hline & Volume $(\mu \mathrm{l})$ \\
\hline Bovine collagen $(6.9 \mathrm{mg} / \mathrm{ml})$ & 271 \\
\hline $2 \times \mathrm{hEHM}$ RPMI (Appendix A1) & 271 \\
\hline $0.1 \mathrm{~N} \mathrm{NaOH}$ & 53 \\
\hline Cell suspension $\left(1.45 \times 10^{6} / \mathrm{EHM}\right)$ & 1511 \\
\hline Total volume & 2106 \\
\hline
\end{tabular}

Table 4. hEHM master mix.

hEHM mixture was cast into circular molds (Figure 12) with an inner/outer diameter of 2/4 mm and supplemented with serum-free hEHM medium (Appendix A1) following an incubation for 1 hour at $37^{\circ} \mathrm{C}$. On culture day 3 , hEHMs were transferred onto static or dynamic stretchers to be exposed to mechanical loading. After culture day 14 or 28 , hEHMs were subjected to isometric force measurements.

\subsection{Isometric force measurement}

EHM functionality was assessed by isometric force measurement in thermostatted organ baths $\left(37{ }^{\circ} \mathrm{C}\right.$; Figure 11C) perfused with carbogen $(95 \% \mathrm{O} 2,5 \% \mathrm{CO})$ to stabilize the $\mathrm{pH}$ at 7.4 as described previously (Zimmermann et al. 2000). EHMs were exposed to electrical stimulation (mEHM and rEHM: $2 \mathrm{~Hz}, \mathrm{hEHM}: 1.5 \mathrm{~Hz}, 200$ $\mathrm{mA}$ ) to contract in Tyrode's solution (Appendix A1). EHMs were firstly preloaded to the maximum length where they generated the maximum force (Lmax according to the Frank-Starling mechanism). EHMs kept at Lmax were exposed to cumulatively increasing calcium concentrations $(0.2 \mathrm{mM}$ to $4 \mathrm{mM})$ to document maximal inotropic capacity and calcium sensitivity. Subsequently, EHMs were exposed to $1 \mu \mathrm{mol} / \mathrm{L}$ isoprenaline and $10 \mu \mathrm{mol} / \mathrm{L}$ carbachol (both from Sigma-Aldrich) at $\mathrm{EC}_{50}$ calcium concentrations. Force of contraction (FOC) data was acquired by BMON 32 and analyzed by AMON 32 software (Föhr Medical Instruments). 


\subsection{Immunostaining and microscopy}

EHMs were fixed with $4 \%$ formaldehyde (FA) overnight at $4{ }^{\circ} \mathrm{C}$ followed by washes in PBS. From this step on, EHM pieces were either directly proceeded to whole mount staining or the complete EHM rings were embedded in $4 \%$ agarose for subsequent vibratome sectioning (Leica vibratome). EHM pieces or sections with $100 \mu \mathrm{m}$ of thickness were blocked in permeabilizing blocking buffer (Appendix A1) overnight at $4{ }^{\circ} \mathrm{C}$. Next, EHMs were treated with primary antibodies (see Appendix A3 for antibody dilutions) in the blocking buffer overnight at $4{ }^{\circ} \mathrm{C}$. Primary antibodies were extensively washed; two times with PBS and one time with blocking buffer. Thereafter, EHMs were incubated in blocking buffer with suitable secondary antibodies (see Appendix A3 for antibody dilutions) and Hoechst $(10 \mu \mathrm{g} / \mathrm{ml}, \mathrm{BD}$ Biosciences, see Appendix $\mathrm{A} 3$ for the dilution) for DNA staining overnight at $4{ }^{\circ} \mathrm{C}$. Following the washing steps; two times with PBS, one time with permeabilizing blocking buffer and one time with PBS, EHMs were mounted on microscope slides and proceeded to confocal microscopy imaging (Zeiss LSM 710).

\subsection{Flow cytometry}

For single cell analyses by flow cytometry EHM had to be dispersed enzymatically and exposed to specific antibody and DNA labeling.

\subsubsection{Dissociation of EHM}

EHMs were incubated in $1 \mathrm{ml}$ of collagenase solution (Appendix A1) for 1 hour at 37 C. The supernatant was collected and the remaining EHM fragments were again exposed to $1 \mathrm{ml}$ of fresh hCM digestion solution (Appendix A1) for 30 minutes at 37 $\mathrm{C}$ to achieve a complete dissociation. Supernatant volume was recorded and subjected to automated cell counting by an electric current exclusion assay (CASY model TTC; Roche) to measure cell number and viability. Thereafter, cells were passed through a $70 \mu \mathrm{m}$ cell strainer to remove clumps and either directly proceeded to flow cytometry for live analysis or fixed with ice-cold $70 \%$ ethanol or $4 \%$ FA at RoT for immunofluorescence staining. 


\subsubsection{Immunostaining for intracellular antigens}

Cells digested from EHM were labeled with primary antibodies directed against sarcomeric $\alpha$-actinin (see Appendix A3 for the antibody dilution) to determine the myocyte amount out of total cell population within the EHM. Firstly, ethanol was removed by a centrifugation step ( $300 \times \mathrm{g}, 5$ minutes) and subsequently cells were permeabilized in permabilizing blocking buffer (Appendix A1) for 10 minutes on ice. Next, cells were incubated with the primary antibody for 45 minutes on ice. Subsequent to washing steps; one time with PBS and one time with the blocking buffer, cells were incubated with a suitable secondary antibody (see Appendix A3) together with Hoechst DNA-binding dye $(10 \mu \mathrm{g} / \mathrm{ml}$, BD Biosciences, see Appendix A3 for the dilution) for 30 minutes in the dark at RoT. Following the washing steps; one time with PBS and one time with the blocking buffer, cells were suspended in $500 \mu \mathrm{l}$ of PBS and subjected to flow cytometry analysis.

\subsubsection{Immunostaining for cell surface proteins}

CPCs and fibroblasts were stained with the corresponding antibodies against cell surface antigens; progenitor/stem cell markers (c-Kit, Sca-1), endothelial cell marker (PECAM1 [CD31]), mesenchymal cell markers (CD90, CD105, PDGFRA), and the common leukocyte marker (CD45). CPCs and fibroblasts were used directly from frozen aliquots. Cells were thawed in culture medium and subsequently washed with PBS. Next, CPCs and fibroblasts were exposed to the indicated antibodies (see Appendix A3 for the antibody dilution) in PBS with $5 \%$ FBS for 15 minutes at $4{ }^{\circ} \mathrm{C}$ in the dark. After an additional washing step with $3 \mathrm{ml}$ of PBS, cells were subjected to flow cytometry (BD LSR II; BD Biosciences).

\subsubsection{Sorting of EHM-derived cells}

Cell mixtures composed of $\mathrm{RFP}^{+}$cardiomyocytes and $\mathrm{GFP}^{+}$non-myocytes (hFFs and hCPCs) obtained from EHM digestion were proceeded to fluorescence-activated cell sorting (FACS) based on their fluorescence labeling. EHM cells were stained with Sytox red dead cell stain (\# S34859, Molecular Probes) and Hoechst DNA-binding dye $(10 \mu \mathrm{g} / \mathrm{ml}$, BD Biosciences, see Appendix A3 for the dilution) to exclude dead cells and cell clusters, respectively, during flow cytometry analysis. RFP ${ }^{+}$myocytes 
and $\mathrm{GFP}^{+}$non-myocytes were recorded within the population gated for live (Sytox negative) and single cells were subsequently sorted (FACSAria II; BD Biosciences) into separate $15 \mathrm{ml}$ polypropylene tubes containing Trizol (Invitrogen) for RNA isolation.

\subsection{Gene expression analysis}

Gene expression analysis was done by semi-quantitative PCR (PCR), quantitative PCR (qPCR) and RNA-sequencing (RNAseq).

\subsubsection{RNA isolation}

Total RNA was isolated using Trizol reagent according to manufacturer's instructions. The amount and purity of RNA (to assess DNA and phenol contaminations respectively; $\mathrm{OD}_{260} / \mathrm{OD}_{280}$ : $\sim 2.0, \mathrm{OD}_{260} / \mathrm{OD}_{230}$ : 2.0-2.2) were identified by the Nanodrop spectrophotometer (Thermo Scientific).

Gene expression analysis in rEHMs was performed by Alberto Izarra (Spanish National Center for Cardiovascular Research - CNIC). rEHM samples were shipped to CNIC on dry ice for RNA isolation followed by PCR for insulin-like growth factor 1 (IGF-1), fibroblast growth factor 2 (bFGF), vascular endothelial growth factor $A$ (VEGF-A) and hepatocyte growth factor (HGF) with mouse specific primers to only amplify transcripts encoding the respective growth factors in the mouse Sca-1-CPC population in rEHMs (Izarra et al. 2014). 


\subsubsection{Reverse transcription}

RNA samples were treated with DNase I (\#04716728001, Roche) according to the protocol described below (Table 5) to remove possible contaminating genomic DNA.

\begin{tabular}{|l|c|}
\hline & Volume \\
\hline RNA $(1 \mu \mathrm{g})$ & $8 \mu \mathrm{l}$ \\
\hline DNase incubation buffer & $1 \mu \mathrm{l}$ \\
\hline DNase $(10 \mathrm{U} / \mu \mathrm{l})$ & $0.2 \mu \mathrm{l}$ \\
\hline
\end{tabular}

Table 5. Composition of DNase treatment.

Dnase treatment reaction was performed at $37^{\circ} \mathrm{C}$ for 20 minutes. Subsequently, $1 \mu \mathrm{l}$ of EDTA solution (1:5 diluted from RNase free EDTA stock solution, $0.2 \mathrm{~mol} / \mathrm{L}$, $\mathrm{pH}$ 8.0) was pipetted into the reaction mix to inhibit DNase activity and incubated at 75 C for 10 minutes.

cDNA synthesis was performed by using High Capacity cDNA Reverse Transcription kit according to the manufacturer's instructions (Applied Biosystems). Briefly, $10 \mu \mathrm{l}$ of Master mix for cDNA synthesis (Table 6) was mixed with $10 \mu \mathrm{l}$ of RNA sample as described above and cDNA reverse transription reaction was run following the program summarized in Table 7.

\begin{tabular}{|l|c|}
\hline & Volume \\
\hline 10x RT buffer & $2 \mu \mathrm{l}$ \\
\hline 25x dNTPs $(100 \mathrm{mM})$ & $0.8 \mu \mathrm{l}$ \\
\hline 10x RT Random Oligo dT primers & $2 \mu \mathrm{l}$ \\
\hline RNase inhibitor & $1 \mu \mathrm{l}$ \\
\hline Diethylpyrocarbonate (DEPC) water & $3.2 \mu \mathrm{l}$ \\
\hline Multiscribe Reverse Transcriptase $(50 \mathrm{U} / \mu \mathrm{l})$ & $1 \mu \mathrm{l}$ \\
\hline
\end{tabular}

Table 6. Composition of cDNA synthesis reaction.

\begin{tabular}{|l|c|c|c|c|}
\hline Temperature & $25^{\circ} \mathrm{C}$ & $37^{\circ} \mathrm{C}$ & $85^{\circ} \mathrm{C}$ & $4^{\circ} \mathrm{C}$ \\
\hline Time & $10 \mathrm{~min}$ & $120 \mathrm{~min}$ & $5 \mathrm{~min}$ & - \\
\hline
\end{tabular}

Table 7. cDNA synthesis protocol. 


\subsubsection{Polymerase chain reaction}

Subsequent to cDNA synthesis, semi-quantitative PCR was performed with primers targeting transcripts from specific genes of interest (Appendix A2) using TaKaRa Ex Taq kit. Briefly, 50 ng of cDNA was mixed with a master mix containing 5' and 3' primers with either $100 \mathrm{nmol} / \mathrm{L}$ or $200 \mathrm{nmol} / \mathrm{L}$ final concentration as indicated in the tables below (Table 8 and 9) for each gene (IGF-1, FGF-2, VEGF-A, HGF, platelet derived growth factor [PDGF], $v$-kit hardy-zuckerman 4 feline sarcoma viral oncogene homolog [c-Kit], Nk2 homeobox 5 [NKX2-5], Gata binding protein 4 [GATA4], Actin, alpha, cardiac muscle 1 [ACTC1], Cardiac troponin I, type 3 [CTnI, PECAM1 [CD31] and Glyceraldehyde-3-Phosphate Dehydrogenase [GAPDH]). The PCR reactions were run on a Veriti $^{\circledR}$ 96-Well Thermal Cycler (Applied Biosystems) as indicated below (Table 10-14).

\begin{tabular}{|l|c|}
\hline & Master Mix \\
\hline Number of samples & $4 \mathrm{x}$ \\
\hline 10x ExTaq Buffer & $5 \mu \mathrm{l}$ \\
\hline dNTP mix & $4 \mu \mathrm{l}$ \\
\hline ExTaq & $0.25 \mu \mathrm{l}$ \\
\hline $5 '$ primer $(10 \mu \mathrm{mol} / \mathrm{L})$ & $1 \mu \mathrm{l}$ (final con. $200 \mathrm{nmol} / \mathrm{L})$ \\
\hline $3^{\prime}$ primer $(10 \mu \mathrm{mol} / \mathrm{L})$ & $1 \mu \mathrm{l}$ (final con. $200 \mathrm{nmol} / \mathrm{L})$ \\
\hline ddH $_{2} \mathrm{O}$ & $36.75 \mu \mathrm{l}$ \\
\hline & $12 \mu \mathrm{l} / \mathrm{sample}$ \\
cDNA sample & $1 \mu \mathrm{l}$ \\
Total volume/sample & $13 \mu \mathrm{l}$
\end{tabular}

Table 8. Composition of the PCR reaction for all targets without CD31. 


\begin{tabular}{|l|c|}
\hline & Master Mix \\
\hline Number of samples & $4 \mathrm{x}$ \\
\hline 10x ExTaq Buffer & $5 \mu \mathrm{l}$ \\
\hline dNTP mix & $4 \mu \mathrm{l}$ \\
\hline ExTaq & $0.25 \mu \mathrm{l}$ \\
\hline $5^{\prime}$ primer $(10 \mu \mathrm{M})$ & $0.5 \mu \mathrm{l}$ (final con. $100 \mathrm{nM})$ \\
\hline $3^{\prime}$ primer $(10 \mu \mathrm{M})$ & $0.5 \mu \mathrm{l}$ (final con. $100 \mathrm{nM})$ \\
\hline $\mathrm{ddH}_{2} \mathrm{O}$ & $37.75 \mu \mathrm{l}$ \\
\hline & $12 \mu \mathrm{l} / \mathrm{sample}$ \\
cDNA sample & $1 \mu \mathrm{l}$ \\
Total volume/sample & $13 \mu \mathrm{l}$
\end{tabular}

Table 9. Composition of the PCR reaction for CD31.

\begin{tabular}{|c|c|c|c|}
\hline Cycle step & Temperature & Time & Cycles \\
\hline Initial denaturation & $95^{\circ} \mathrm{C}$ & $60 \mathrm{sec}$. & $1 x$ \\
\hline Denaturation & $95^{\circ} \mathrm{C}$ & $15 \mathrm{sec}$. & \multirow{3}{*}{$35 x$} \\
\hline Annealing & $60^{\circ} \mathrm{C}$ & $15 \mathrm{sec}$. & \\
\hline Extension & $72^{\circ} \mathrm{C}$ & $30 \mathrm{sec}$. & \\
\hline Final extension & $72^{\circ} \mathrm{C}$ & $300 \mathrm{sec}$. & $1 x$ \\
\hline
\end{tabular}

Table 10. PCR program for FGF-2/VEGF-A/PDGF/GAPDH.

\begin{tabular}{|c|c|c|c|}
\hline Cycle step & Temperature & Time & Cycles \\
\hline Initial denaturation & $95^{\circ} \mathrm{C}$ & $60 \mathrm{sec}$. & $1 x$ \\
\hline Denaturation & $95^{\circ} \mathrm{C}$ & $15 \mathrm{sec}$. & \multirow{3}{*}{$35 x$} \\
\hline Annealing & $60^{\circ} \mathrm{C}$ & $30 \mathrm{sec}$. & \\
\hline Extension & $72{ }^{\circ} \mathrm{C}$ & $60 \mathrm{sec}$. & \\
\hline Final extension & $72^{\circ} \mathrm{C}$ & $300 \mathrm{sec}$. & $1 x$ \\
\hline
\end{tabular}

Table 11. PCR program for IGF-1/GATA4/ACTC1/CTnl. 


\begin{tabular}{|c|c|c|c|}
\hline Cycle step & Temperature & Time & Cycles \\
\hline Initial denaturation & $95^{\circ} \mathrm{C}$ & $60 \mathrm{sec}$. & $1 x$ \\
\hline Denaturation & $95^{\circ} \mathrm{C}$ & $30 \mathrm{sec}$. & \multirow{3}{*}{$35 x$} \\
\hline Annealing & $56^{\circ} \mathrm{C}$ & $30 \mathrm{sec}$. & \\
\hline Extension & $72^{\circ} \mathrm{C}$ & $60 \mathrm{sec}$. & \\
\hline Final extension & $72^{\circ} \mathrm{C}$ & $300 \mathrm{sec}$. & $1 x$ \\
\hline
\end{tabular}

Table 12. PCR program for NKX2-5.

\begin{tabular}{|c|c|c|c|}
\hline Cycle step & Temperature & Time & Cycles \\
\hline Initial denaturation & $95^{\circ} \mathrm{C}$ & $60 \mathrm{sec}$. & $1 x$ \\
\hline Denaturation & $95^{\circ} \mathrm{C}$ & $30 \mathrm{sec}$. & \multirow{3}{*}{$30 x$} \\
\hline Annealing & $60^{\circ} \mathrm{C}$ & $30 \mathrm{sec}$. & \\
\hline Extension & $72^{\circ} \mathrm{C}$ & $60 \mathrm{sec}$. & \\
\hline Final extension & $72^{\circ} \mathrm{C}$ & $300 \mathrm{sec}$. & $1 x$ \\
\hline
\end{tabular}

Table 13. PCR program for CD31/c-Kit.

\begin{tabular}{|c|c|c|c|}
\hline Cycle step & Temperature & Time & Cycles \\
\hline Initial denaturation & $95^{\circ} \mathrm{C}$ & $60 \mathrm{sec}$. & $1 \mathrm{x}$ \\
\hline Denaturation & $95^{\circ} \mathrm{C}$ & $30 \mathrm{sec}$. & \multirow{3}{*}{$35 x$} \\
\hline Annealing & $55^{\circ} \mathrm{C}$ & $30 \mathrm{sec}$. & \\
\hline Extension & $72^{\circ} \mathrm{C}$ & $60 \mathrm{sec}$. & \\
\hline Final extension & $72^{\circ} \mathrm{C}$ & 300 sec. & $1 x$ \\
\hline
\end{tabular}

Table 14. PCR program for HGF.

\subsubsection{Agarose gel electrophoresis}

Amplified DNA fragments by PCR were visualized by agarose gel electrophoresis for gene expression analysis. 2\% agarose gels (ultra-pure agarose; AppliChem) were prepared in TAE buffer (Appendix A1). The agarose gel mixture was heated in a microwave until agarose particles were completely dissolved. Prior to gel polymerization ethidium bromide was added (final concentration $0.2 \mu \mathrm{g} / \mathrm{ml}$ ) for visualization of the DNA under UV light. Agarose gels were poured into standard casting trays. Upon polymerization, agarose gels were loaded with the cDNA 
samples mixed with DNA gel loading dye (\#R0611, ThermoFisher Scientific) and a DNA ladder (Gene ruler $^{\text {TM }} 100$ bp Plus DNA ladder, \#SM0321, ThermoFisher Scientific). Elecrophoretic separation was at $90 \mathrm{~V}$ for 1-2 hours at RoT.

\subsubsection{Quantitative PCR}

To analyze the expression of cardiac genes in flow cytometry sorted cells $(2550 \pm 634$ $\mathrm{RFP}^{+}$cells, $1349 \pm 348 \mathrm{GFP}^{+}$cells per reaction, from three experiments) quantitative PCR (qPCR) was performed using Fast SYBR Green Master Mix kit (Applied Biosystems) according to manufacturer's instructions. Briefly, 5-10 ng cDNA was mixed with a master mix containing 5' and 3' primers (50 nmol/L each; Table 15). qPCR was performed in ABI PRISM 7900HT Fast Real-Time PCR system (Applied Biosystems) on 384-well plate format according to the PCR program shown in Table 16. qPCR analysis was performed using $\triangle \triangle \mathrm{Ct}$ method and $G A P D H$ was used as the reference gene for normalization (Livak and Schmittgen 2001).

\begin{tabular}{|c|c|}
\hline & Master Mix \\
\hline Number of samples & $30 x$ \\
\hline $\mathrm{ddH}_{2} \mathrm{O}$ & $108 \mu \mathrm{l}$ \\
\hline 5' primer $(10 \mu \mathrm{mol} / \mathrm{L})$ & $1.25 \mu \mathrm{l}$ \\
\hline 3' primer $(10 \mu \mathrm{mol} / \mathrm{L})$ & $1.25 \mu \mathrm{l}$ \\
\hline Sybr Master Mix & $150 \mu \mathrm{l}$ \\
\hline $\begin{array}{l}\text { cDNA sample } \\
\text { Total volume/sample }\end{array}$ & $\begin{array}{c}9 \mu \mathrm{l} / \text { sample } \\
1 \mu \mathrm{l} \\
10 \mu \mathrm{l}\end{array}$ \\
\hline
\end{tabular}

Table 15. Composition of qPCR reaction.

\begin{tabular}{|l|l|c|}
\hline Initial denaturation & $95^{\circ} \mathrm{C}$ & $1 \mathrm{x}$ \\
\hline Denaturation & $95^{\circ} \mathrm{C}$ & \multirow{2}{*}{$40 \mathrm{x}$} \\
\cline { 1 - 2 } Annealing & $60^{\circ} \mathrm{C}$ & \\
\hline Extension & $60^{\circ} \mathrm{C}$ & $1 \mathrm{x}$ \\
\hline Final extension & $60^{\circ} \mathrm{C}$ & $1 \mathrm{x}$ \\
\hline Inactivation & $95^{\circ} \mathrm{C}$ & \\
\hline
\end{tabular}

Table 16. qPCR protocol. 


\subsubsection{RNA sequencing}

RNA was isolated as described in section 2.7.1 and the quality was assessed with the Agilent Bioanalyzer 2100. Total RNA was subjected to library preparation (TruSeq Stranded Total RNA Sample Prep Kit from Illumina) and RNA-sequencing on an Illumina HighSeq-2000 platform (SR 50 bp; >25 Mio reads/sample). Sequence images were transformed with the Illumina software BaseCaller to bcl files, which were demultiplexed to fastq files with CASAVA (v1.8.2). Fastq files were mapped to GRCh38/hg38 using STAR 2.4 or TopHat2 (Kim et al. 2013) and Reads Per Kilobase of transcript per Million (RPKM) were calculated based on the Ensembl transcript length as extracted by biomaRt (v2.24) or Fragments Per Kilobase of transcript per Million (FPKM) calculated by Cufflinks (Trapnell et al. 2012). We only considered "protein_coding" transcripts for further analysis. Gene ontology (GO) analysis was performed through DAVID (Huang da et al. 2009). To determine cardiomyocyte, fibroblast and CPC specific genes the following algorithm was applied: (1) normalized counts of purified hES2.R26-derived cardiomyocytes $(n=3)$ and fibroblasts from two different sources (foreskin and heart; $n=3$ from each source) and human GFP+CPCs $(n=3)$ were pooled and the differentially expressed genes (DEG, $p<0.05)$ between cardiomyocyte, fibroblast and CPC pools determined; (2) log2 changes of DEG were calculated and genes omitted with a log2 difference lower than mean log2 of all cardiomyocyte genes; (3) resulting cardiomyocyte-, fibroblast-, and CPC-enriched genes were screened for RPKM values in adult healthy heart. Transcribed genes with RPKM $<1$ in adult heart were omitted.

\subsection{Cell based models of cardiomyocyte hypoxia}

To investigate possible cardioprotective activity of CPCs, we developed a new EHM injury model based on hypoxia/reoxygenation $(\mathrm{H} / \mathrm{R})$ or chronic hypoxia. Embryonic stem cells from ODD-Luc mice (refer to 2.1.2) or a newly generated human ODD-Luc reporter embryonic stem cell line (refer to 2.11) were used as cardiomyocyte sources for EHM construction according to the protocols outlined above. 


\subsubsection{Imaging hypoxia in mouse ODD-Luc cardiomyocytes}

ODD-Luc fusion protein has been shown to be responsive to hypoxia and can be used as a noninvasive bioluminescence imaging technique to monitor the temporal kinetic of endogenous HIF-1a regulation in different cell types (Moroz et al. 2009, Viola et al. 2008). Being the main regulatory protein for adaptation of cellular response to hypoxia, identification of HIF-1a regulation may provide mechanistic insight into diseases like ischemia/reperfusion injury. To visualize and analyze the response to hypoxia in cardiomyocytes, we utilized the ODD-Luc $x$ a-MHC-NeoR mESC line to give rise to ODD-Luc cardiomyocytes which ubiquitously express HIF1a-ODD-Luc fusion protein. ODD-Luc cardiomyocytes were incubated in hypoxia chamber (Invivo 400 workstation, Ruskinn, UK) under $1 \% \mathrm{O}_{2}$ for 8-72 hours. After hypoxia treatment, ODD-Luc CMs were lysed with a lysis buffer (Dual-Luciferase ${ }^{\circledR}$ Reporter Assay Systems, Promega) diluted 1:5 with $\mathrm{dd}_{2} \mathrm{O}$ water and supplemented with protease and phosphatase inhibitors (both diluted in 1:1000 dilution, see Appendix A1 for stock solutions) inside the hypoxia chamber. Thereafter, the enzymatic reaction depicted in Figure $\mathbf{1 3}$ was performed according to manufacturer's protocol using Centro LB 960 Microplate Luminometer (BERTHOLD Technologies) and Microwin 2000 software (Informer Technologies).

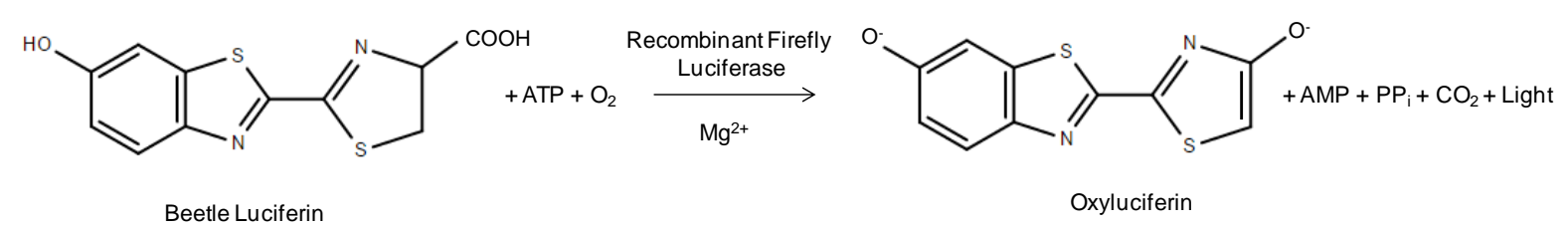

Figure 13. Bioluminescent reaction catalyzed by firefly luciferase. Luciferase activity assay involves oxidation of luciferin substrate in the presence of $\mathrm{O}_{2}$, ATP and $\mathrm{Mg}^{2+}$. Schematic adapted from the Promega user manual.

\subsubsection{Imaging hypoxia in mouse ODD-Luc EHM}

In the next step, ODD-Luc hypoxia reporter CMs (70\%) and mEFs (30\%) were utilized to construct ODD-Luc mouse EHM to monitor hypoxia at a tissue level. ODDLuc EHMs were incubated in $1 \% \mathrm{O}_{2}$ for 1-72 hrs. Thereafter, ODD-Luc EHMs were transferred into PBS containing $1 \mathrm{mg} / \mathrm{ml}$ of luciferin (Caliper life sciences) and immediately visualized for bioluminescence development using LAS-3000 
luminesence image analyzer (Fuji). Analysis of luminescence intensity was done by Image J software.

\subsubsection{Generation of ODD-Luc human embryonic stem cell line}

The plasmid ODD-Luciferase-pcDNA3 was a kind gift from the Kaelin lab (DanaFarber/Harvard Cancer Center). ODD-Luc transgene was cloned from the original plasmid into a pAAVS1 vector under the control of CAG promoter resulting in PAAVS1-CAG-ODD-Luc-EF1a-GFP donor vector. ODD-Luc hES2 cells were generated via transfection with the ODD-Luc expressing pAAVS1 donor vector in conjuction with pAAVS1 Trancription activator-like effector-nuclease (TALEN) pair plasmids following the principle as described in Figure 14. Briefly, TALE nucleases encoded by TALEN pair plasmids recognize and cut specific DNA sequences in the AAVS1 safe harbor genomic locus, facilitating homologous recombination of the transgene flanked by TALE recognizing sequences. The donor vector was constructed by Dr. Claudia Noack (Institute of Pharmacology and Toxicology, University Medical Center Göttingen) and transfection of hESCs was kindly performed by Krasimira Sharkova (Institute of Pharmacology and Toxicology, University Medical Center Göttingen). Next, puromycin selected ODD-Luc expressing hES cells (ODD-Luc hESCs) were differentiated into human cardiomyocytes (hCMs) giving rise to the formation of ODD-Luc hCMs. 


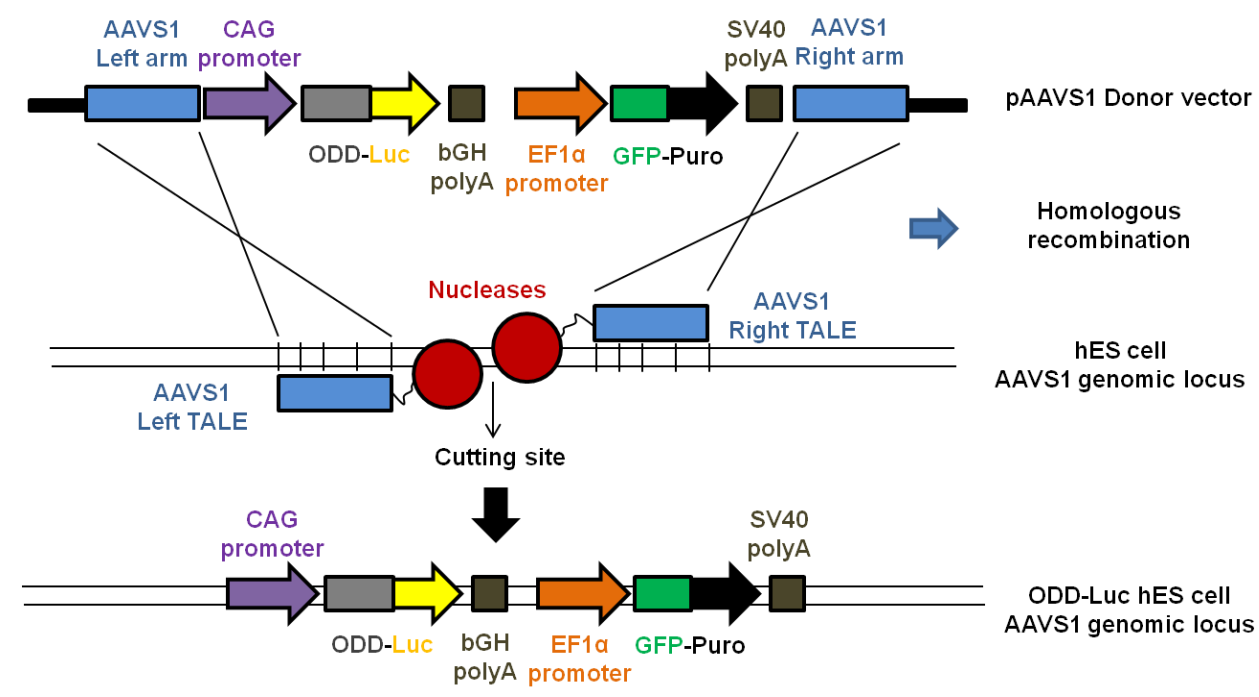

Figure 14. ODD-Luc hES cells generated using TALEN technology. pAAVS1 donor vector encoding for ODD-Luc under CAG promoter control and the GFP-puromycin (puro) fusion protein under EF-1a promoter control flanked by AAVS1 specific DNA sequences. TALE nucleases recognize and cut specific DNA sequences in AAVS1 genomic locus in hES cells, leading typically to homologous recombination of gene of interest in the targeted site.

\subsubsection{Hypoxia imaging in ODD-Luc human cardiomyocytes}

Human cardiomyocytes differentiated from ODD-Luc hESCs were dissociated as described above (section 2.1.3) and seeded on Matrigel ${ }^{\mathrm{TM}}$ (Corning, 1:30 diluted in PBS)-coated 6-well plates with a seeding density of $0.5 \times 10^{6}$ cells/well in hCM medium (Appendix A1). After 5-7 days of metabolic selection in hCM selection medium (Appendix A1) to reduce non-myocyte content, cardiomyocytes were cultured in $21 \%$ or $1 \% \mathrm{O}_{2}$ for 4 hours and subjected to Luc-signal measurements as described above (section 2.8.1).

\subsubsection{Hypoxia/Reoxygenation injury in human EHM}

hEHMs generated from hCMs $(70 \%)$ and $\mathrm{hFFs}(30 \%)$, so called hFF-EHMs were exposed to $1 \% \mathrm{O}_{2}$ for 8-120 hours in DMEM SF-B27 medium (Appendix A1). After hypoxia exposure, hEHMs were incubated in $21 \% \mathrm{O}_{2}$ in freshly prepared DMEM SFCMR medium (Appendix A1) to induce reoxygenation. In order to analyze cardioprotective paracrine effects of hCPCs and hFFs, hEHMs were treated with hCPC- or hFF-Conditioned medium (ConM; Appendix A1) during reoxygenation. 
After $H / R$ injury, cardiomyocyte content, structure and function were assessed. In addition, lactate dehydrogenase $(\mathrm{LDH})$ release was assessed according to the manufacturer's protocol (Lactate dehydrogenase activity assay kit, \#MAK066, SigmaAldrich) to estimate the amount of cell death.

\subsubsection{Chronic hypoxia injury in human EHM}

Next, we investigated the possible cardioprotective effect of hCPCs under chronic hypoxia stimulation for 120 hours in $1 \% \mathrm{O}_{2}$. We developed another hEHM model mainly composed of hCMs and hFFs partially included with hCPCs, so called $\mathrm{hFF}+\mathrm{hCPC}-\mathrm{EHM}$ (input cell numbers $\left[\times 10^{6}\right]$ per $\mathrm{hEHM}$; hCM/hFF/hCPC: 0.84/0.36/0.09). hFF- and hFF+hCPC-EHMs were incubated in DMEM SF-B27 medium (Appendix $\mathrm{A} 1$ ) at $21 \%$ or $1 \% \mathrm{O}_{2}$ without any medium change for 120 hours. Afterwards, hEHMs were subjected to isometric force measurements. In addition, myocyte content, structure and function were analyzed in hEHM co-cultures.

\subsection{Western blot analyses}

Response of human embryonic stem cell-derived cardiomyocytes (hCMs) to hypoxia was assessed by detection of HIF-1a stabilization using western blot technique.

\subsubsection{Protein isolation}

hCMs were seeded in Matrigel ${ }^{\mathrm{TM}}$ (Corning, 1:30 diluted in $1 \mathrm{X}$ PBS)-coated 6-well plates within the density of $0.3 \times 10^{6}$ cells/well in $\mathrm{hCM}$ medium (Appendix A1) and subjected to $1 \% \mathrm{O}_{2}$ for $0-72$ hours. Cells were lysed with $500 \mu \mathrm{l}$ lysis buffer (DualLuciferase $^{\circledR}$ Reporter Assay Systems, Promega)/well as described above (see section 2.8.1), followed by a short centrifugation step $(16,000 \times \mathrm{g}, 10$ seconds, at 4 C) to pellet cell debris. The supernatant was used for the Western blotting.

\subsubsection{Bradford assay}

Protein concentration was measured using the Bradford assay. Serial dilutions (in $\mu \mathrm{g} / \mathrm{ml}: 0,0.312,0.625,1.25,2.5,5)$ of bovine serum albumin (BSA; stock concentration of $1 \mathrm{mg} / \mathrm{ml}$ ) in distilled water were used as reference. Absorbance of samples $(5 \mu \mathrm{l})$ supplemented with Bradford dye $(95 \mu \mathrm{l}$ of 1:10 in sterile water diluted 
dye) was measured in a 96-well plate at $595 \mathrm{~nm}$ (FlexStation 3 Multi-Mode Microplate Reader, Molecular Devices). The accuracy of the measurement was defined based on $R^{2}$ of the standard curve. The experimental $R^{2}$ value for this test was 0.96 ( $n=1$ experiment). Protein concentration was calculated based on the slope intercept formula.

\subsubsection{SDS-gel electrophoresis}

Proteins were seperated using $12 \%$ seperating gel (Appendix A1). The separating gel was poured in between two glass plates. The upper part of the gel was covered with isopropanol during polymerization. Upon polymerization of the gel, isopropanol was removed and washed with distilled water. Next, a stacking gel (Appendix A1) was poured on top of the separating gel with a comb placed inside. The comb was carefully removed upon polymerization.

$20 \mu \mathrm{g}$ of protein was mixed with $6 \mathrm{x}$ Laemmli loading buffer (Appendix A1) and kept at $95{ }^{\circ} \mathrm{C}$ for 3 minutes prior to loading. Samples (30-40 $\left.\mu \mathrm{l}\right)$ and a standard protein ladder sample (The Precision Plus ${ }^{\mathrm{TM}}$ Protein Kaleidoscope ${ }^{\mathrm{TM}}$ protein, BIO-RAD) were loaded and run in Running buffer (Appendix A1) for $\sim 2$ hours at $100 \mathrm{~V}$ and RoT. Next, proteins were transferred from the SDS-gel to a polyvinylidene fluoride (PVDF) by electroblotting in Transfer Buffer for 1.5 hours at $100 \mathrm{~V}$ and $4{ }^{\circ} \mathrm{C}$.

\subsubsection{Immunoblotting}

PVDF membranes, after proteins transfer, were incubated with blocking buffer (Appendix A1) for 1 hour at RoT. After a short washing step in PBS-T (PBS with $0.1 \%$ Tween-20), PVDF membranes were incubated with primary antibodies (HIF-1 $\alpha$ from BD Transduction and $\beta$-actin from Sigma-Aldrich; see Appendix A3 for antibody dilutions) in blocking buffer overnight at $4{ }^{\circ} \mathrm{C}$. After three additional washing steps in PBS-T, membranes were incubated with appropriate secondary antibodies (IgGHRP-conjugated, Dako, see Appendix A3 for antibody dilution) in blocking buffer overnight at $4{ }^{\circ} \mathrm{C}$. After three washes in PBS-T for 15 minutes each, membranes were incubated with the substrate (SuperSignal ${ }^{\circledR}$ West Femto Trial Kit, Themofischer scientific) which catalyzes the activity of horseradish peroxidase to generate a chemiluminesence signal. The chemiluminesence signal was detected by a 
VersaDoc ${ }^{\mathrm{TM}} \mathrm{XR}$ Imaging System and the images were analzyed using Image Lab software from BioRad.

\subsection{Quantification of lactate release}

hCMs were seeded into 24-well plates (62.500 cells/well) in hCM medium (Appendix A1). Culture medium was changed with DMEM SF-B27 medium (Appendix A1) before incubation at $1 \% \mathrm{O}_{2}$ for $0-72$ hours. Prior to analysis, medium was collected in $1.5 \mathrm{ml}$ polypropylene tubes, centrifuged for $20-25$ minutes at $16,000 \times \mathrm{g}$ to remove any cell debris. Supernatant was transferred into a new polyprolylene tube. $100 \mu \mathrm{l}$ of medium was added into the master mix prepared according to manufacturer's instructions (L-lactic acid UV-method, Boehringer/R-Biopharm). The complete mixture was transferred into plastic cuvettes to initiate the enzymatic reactions (Figure 15) and subjected to spectrophotometric measurement of NADH at $340 \mathrm{~nm}$ (Centro LB 960 Microplate Luminometer, BERTHOLD Technologies). Lactic acid concentration was calculated based on the equation described in the related protocol.
(1) L-Lactate + NAD +

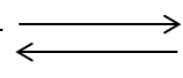
pyruvate $+\mathrm{NAD}^{+}+\mathrm{H}^{+}$
(2) Pyruvate + L-glutamate
L-alanine + 2-oxoglutarate

Figure 15. Lactic acid catalyzing reaction. 1 L-Lactate is oxidized to pyruvate in the presence of $\mathrm{NAD}^{+}$and L-Lactate dehydrogenase (L-LDH). 2 Pyruvate from reaction 1 is trapped in the presence of L-glutamate and Glutamate-Pyruvate transaminase, leading the eqilibrium in reaction 1 to be displaced in favor of pyruvate and $\mathrm{NADH}^{+}$.

\subsection{Quantification of intracellular ATP}

hCMs were seeded in matrigel (Corning, 1:30 diluted in 1x PBS)-coated 96-well luminometer plate within the density of $2.5 \times 10^{4}$ cells/well in hCM medium. Cells were put into $1 \% \mathrm{O}_{2}$ for $0-72$ hours following medium change with DMEM SF-B27 medium (Appendix A1). Prior to analysis, ATP standards were prepared in each 96well plate with the concentrations ranging from $1 \mu \mathrm{M}$ to $10 \mathrm{nM}$ by serial ten fold dilutions. $100 \mu \mathrm{l}$ of Cell titer Glo Reagent (mixture of Luciferin substrate and Luciferase) were added on top of the medium. The luminescence signal generated 
was directly proportional to the amount of ATP present. ATP amount was calculated based on the ATP standard curve. All steps were performed according to manufacturer's instructions (CellTiter-Glo ${ }^{\circledR}$ Luminescent Cell Viability Assay, Promega).

\subsection{Statistics}

Data are presented as arithmetic mean with standard error of the mean (SEM). Statistical analyses were done using two-tailed unpaired Student's $t$-test, one-way ANOVA, and two-way ANOVA with indicated post hoc tests using GraphPad Prism5 program. $P$ value smaller than $0.05\left({ }^{*} p<0.05\right)$ was considered significant. 


\section{Results}

\subsection{Characterization of CPCs in monolayer culture}

Cardiac progenitor cells (CPCs) derived from adult mouse (mCPCs) or adult human heart (hCPCs) were characterized for their expression of specific cell-type markers using PCR and flow cytometry. Additionally, transcriptome profiling of hCPCs was performed by RNA-sequencing (RNAseq) to gain broader insight into their origin and biological activity.

\subsubsection{Mouse CPCs show a mesenchymal phenotype}

Two previously reported types of CPCs from adult mouse heart, selected by surface markers - (1) v-kit hardy-zuckerman 4 feline sarcoma viral oncogene homolog (c-Kit; c-Kit-mCPCs; kindly provided by Mark Sussmann; San Diego State University; (Fischer et al. 2009)) and (2) lymphocyte antigen 6a (LY6A; Sca-1-mCPCs; kindly provided by Antonio Bernad; CNIC-Madrid; (Izarra, 2014 \#95)) - were characterized. c-Kit expression could not be confirmed by PCR (Figure 16B) or flow cytometry (Figure 16C) in either of the tested mCPCs in monolayer culture. Flow cytometry showed that most of the c-Kit-selected mCPCs were positive for Sca-1 and the mesenchymal cell markers platelet derived growth factor receptor, alpha polypeptide (PDGFRA) and endoglin (CD105; Figure 16C). Additional PCR analyses showed that both mCPC types (c-Kit- and Sca-1-CPCs) also express discoidin domain receptor tyrosine kinase 2 (Ddr2), indicating their mesenchymal origin (Figure 16B). The absence of the cardiomyocyte development implicated transcription factor, Nkx25 suggested that both of these cell types were not committed towards a cardiomyocyte fate (Figure 16B). 
A
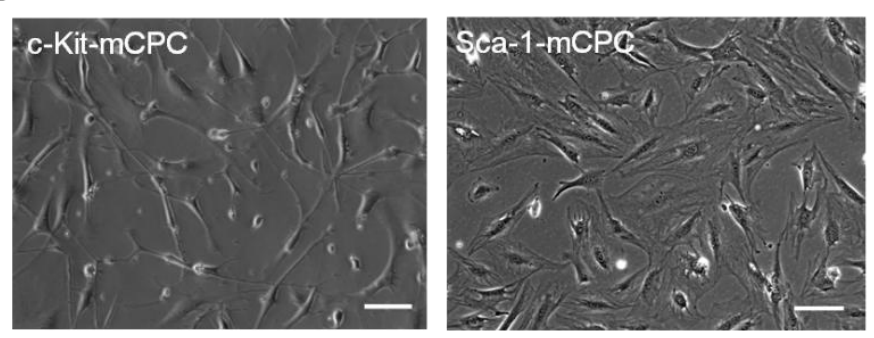

B

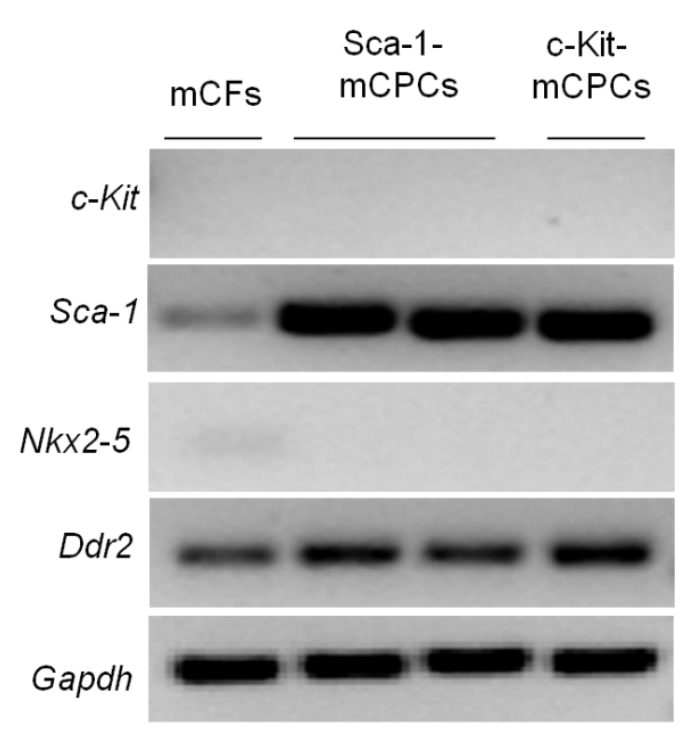

C
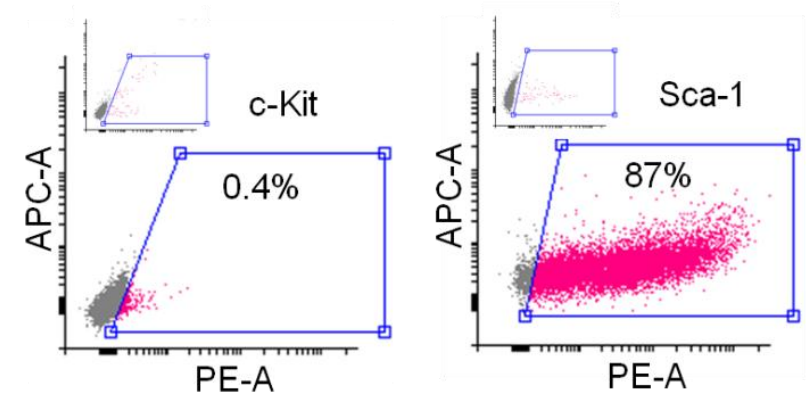

PE-A

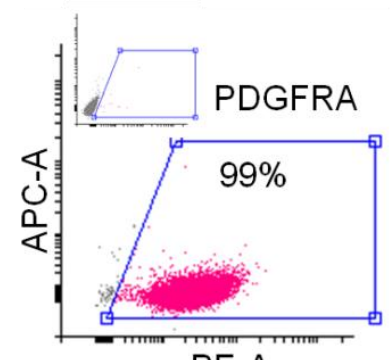

PE-A

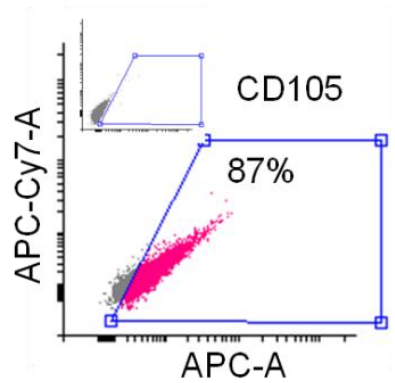

Figure 16. Characterization of mCPCs. A Morphology of c-Kit- and Sca-1-mCPCs in monolayer culture. B RNA expression of stem cell markers C-Kit and Sca-1, early cardiac marker Nkx2-5 and fibroblast marker Ddr2 in mouse cardiac fibroblasts (mCFs), Sca-1-mCPC and c-Kit-mCPC by PCR. Glyceraldehyde-3-phosphate dehydrogenase (Gapdh) was used as housekeeping gene for loading control. C Flow cytometry characterization of c-Kit-mCPCs for the expression of the "stem cell markers" c-Kit and Sca-1 (LY6A) as well as mesenchymal cell markers PDGFRA and CD105.

\subsubsection{Human CPCs are morphologically distinct from fibroblasts}

Human CPCs (hCPCs) isolated based on the c-Kit surface marker were delivered in frozen aliquots from Coretherapix (Spain) as part of a collaboration within the EU FP7 CARE-MI Consortium. hCPCs were used immediately after thawing for characterization without additional culture in our laboratory to avoid technical artifacts that could modify the expression profile and original phenotype of the cells. hCPCs were characterized in direct comparison with human fibroblasts (hFFs: human foreskin fibroblasts) and human embryonic stem cell-derived cardiomyocytes (hCMs). 
hCPCs and hFFs showed distinct cell morphologies in vitro. hCPCs seemed to display a more spreaded phenotype with frequent protrusions, while hFFs were more elongated (Figure 17). hCPCs and hFFs with stable expression of GFP were used.
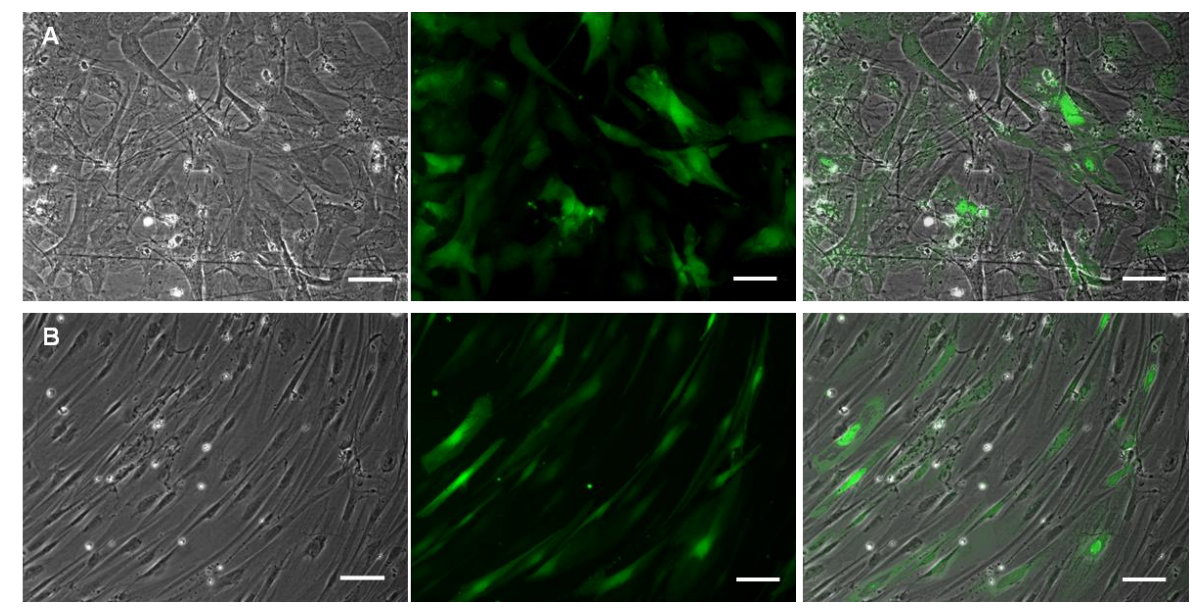

Figure 17. Morphologically distinct phenotypes in hCPC and hFF monolayer cultures. A hCPCs (plated after thawing upon receipt from Coretherapix) and B hFFs in brightfield (left), GFP fluorescence (middle) and merged (right) images. Scale bars: $100 \mu \mathrm{m}$.

\subsubsection{Human CPC pools contain mesenchymal/endothelial cells}

Similar as for the mCPCs, there was no c-Kit expression detected in hCPC on mRNA level, although they were originally isolated based on the c-Kit receptor. Interestingly, hFFs were positive for c-Kit on the mRNA level, but also not by flow cytometry. hCPCs showed no expression of the definitive cardiac markers cardiac troponin I (TNN/3) and cardiac actin (ACTC1). NKX2-5 was also not detected in contrast to GATA4 and the endothelial cell marker PECAM1 (Figure 18). 


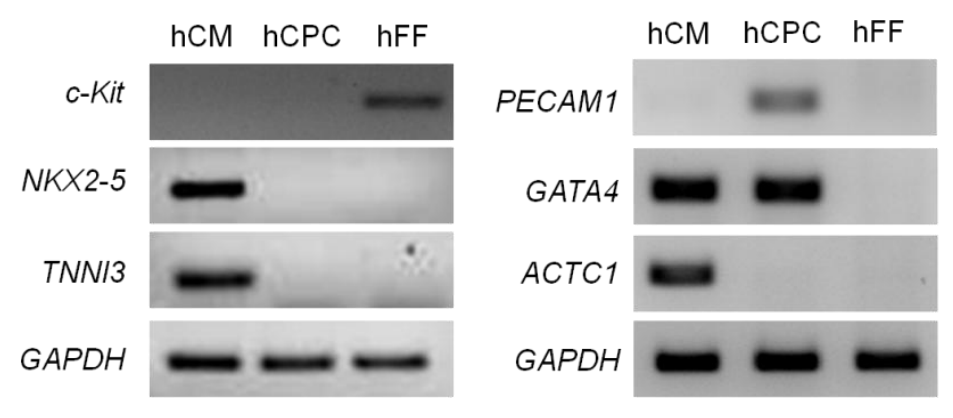

Figure 18. Expression of cardiac cell markers in hCPCs. RNA expression of the "stem cell" marker c-Kit, cardiac transcription factors NKX2-5 and GATA4, cardiac markers TNNI3 and ACTC1, endothelial cell marker PECAM1 in hCMs, hCPCs and hFFs by PCR. GAPDH was used as housekeeping gene for loading control.

Next, flow cytometry analysis of cell surface receptor expression confirmed that hCPCs lack c-Kit receptor expression (Figure 19). In line with the PCR data (Figure 19), a small fraction of hCPCs expressed PECAM1 (7.3 $\pm 3.7 \%, n=3 /$ group). In addition, a larger fraction of the hCPCs were decorated by CD90 $(35.6 \pm 9.9 \%$, $\mathrm{n}=3 /$ group) and CD105 (60\%, $\mathrm{n}=1 /$ group), indicating a mesenchymal phenotype (Figure 19). Given the fact that CD105 plays an important role in angiogenesis (Duff et al. 2003), partial expression of CD105 together with vessel associated marker such as PECAM1 in hCPCs might give a hint for angioblast-like characteristics of hCPCs in the heart. The absence of CD45 positive cells suggested there is no detectable leukocyte contamination (Figure 19). 


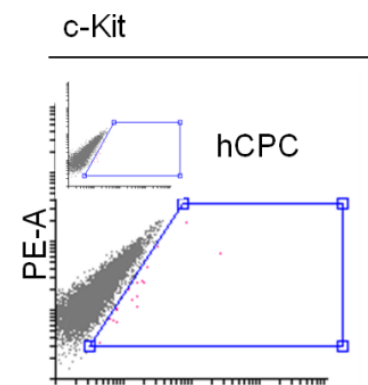

Pacific Blue-A

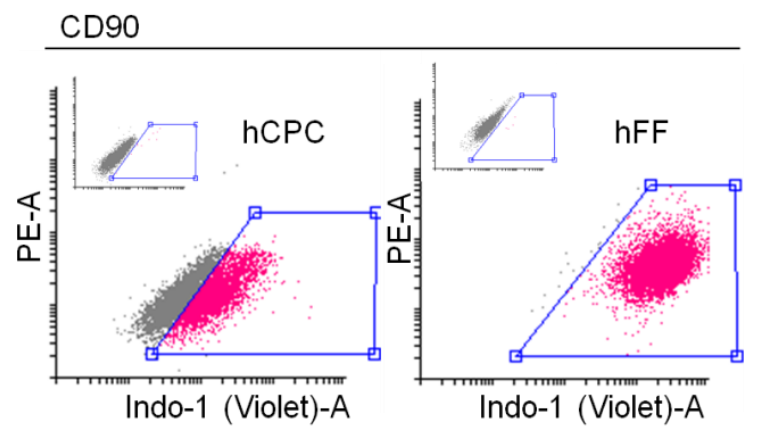

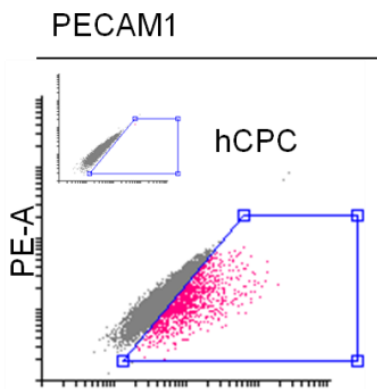

FITC-A

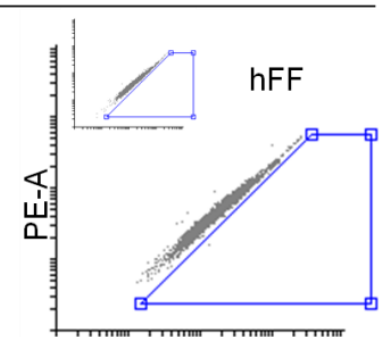

FITC-A
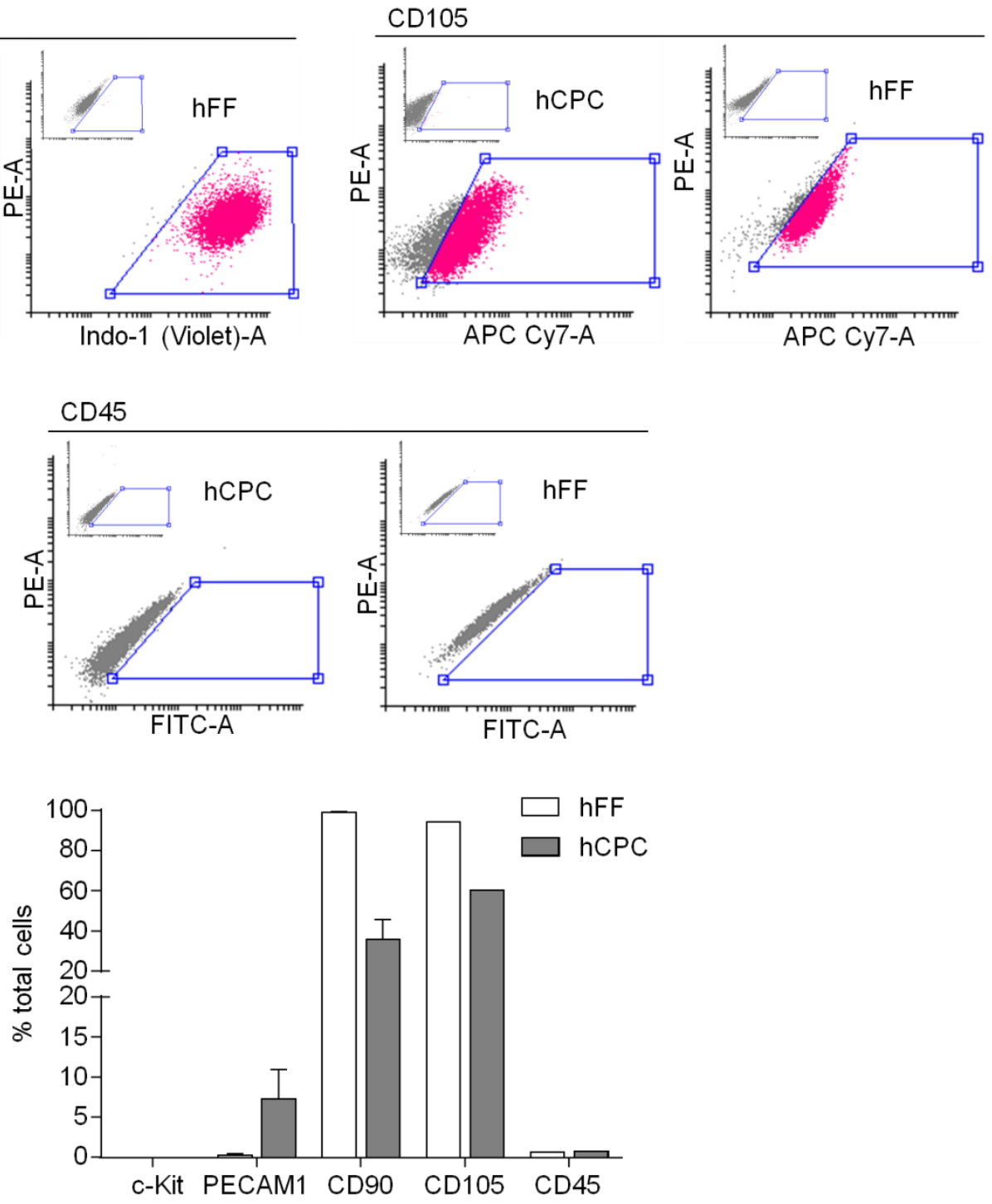

Figure 19. Flow cytometry characterization of hCPCs. Immunophenotype analysis for the expression of stem cell marker $c-K i t$ ( $n=2 /$ group), endothelial cell marker PECAM1 ( $n=3 /$ group), panleukocyte cell marker CD45 ( $n=2 /$ group), mesenchymal cell markers CD90 ( $n=3 /$ group) and CD105 ( $n=1 /$ group) in hFFs and hCPCs. The bar graph below shows quantification of percentage of positively stained cells for the markers mentioned above. Insets show the respective flow cytometry analyses after exposure to suitable isotype control antibodies. 
Collectively, the PCR and flow cytometry data suggest that hCPCs comprise a heterogeneous population, composed of mesenchymal and endothelial cells. GATA4 expression might indicate some cardiomyogenic potential within the hCPC population (Figure 18).

\subsubsection{Human CPCs exhibit a unique transcriptome profile}

The initial PCR and flow cytometry analyses indicated that hCPCs differ from human foreskin fibroblasts (hFFs) and comprise a heterogeneous population of mesenchymal/endothelial cells. RNAseq was performed to further corroborate and extend these findings. Transcriptomes from hCPCs, human cardiac fibroblasts (hCFs), human embryonic stem cell-derived cardiomyocytes (hCMs) and hFFs were compared (Figure 20). Principal component analysis (PCA) showed that hCPCs differ from fibroblasts (hFFs and hCFs) and cardiomyocytes, confirming their unique identity (Figure 20A). Comparative transcriptome analysis between hCPCs and fibroblasts revealed a set of differentially expressed genes (DEGs) specific for each cell type (hCPC: 1,528, hFF: 1,612, hCF: 1,270 genes; Figure 20B). Genes specifically expressed in hCPCs differently than hCFs and hFFs (1,528 genes highlighted with orange color in the Venn diagram) were clustered into certain functional groups by gene ontology analysis suggesting that hCPCs actively transcribe unique plasma membrane, cell adhesion, cytoskeleton, and extracellular proteins (Figure 20C). All hCPC specific DEGs with their classifications are summarized in Appendix A5. 


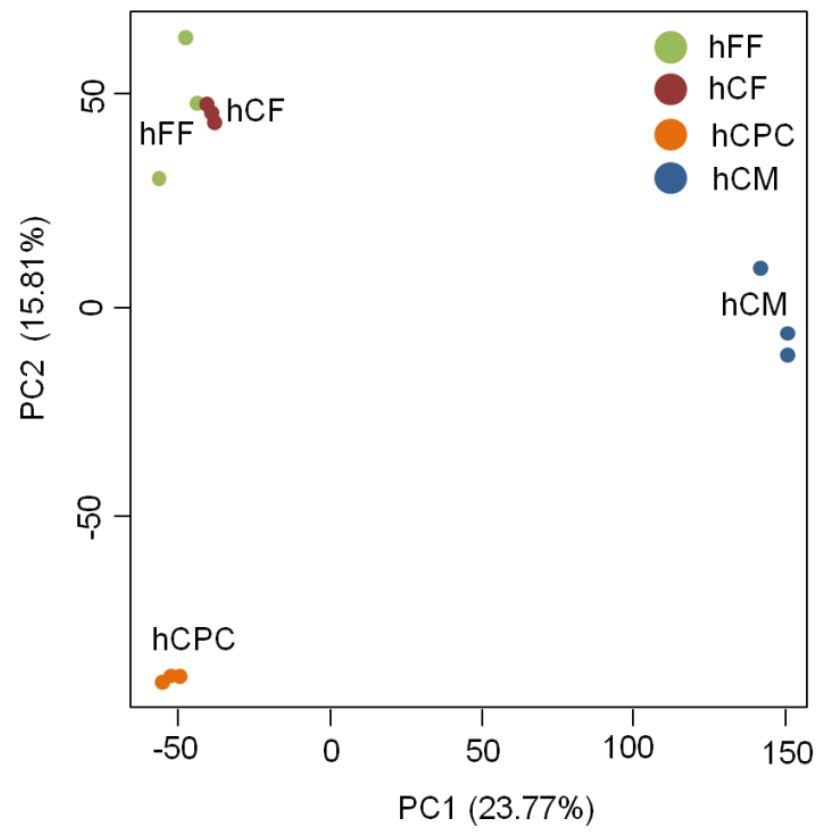

B

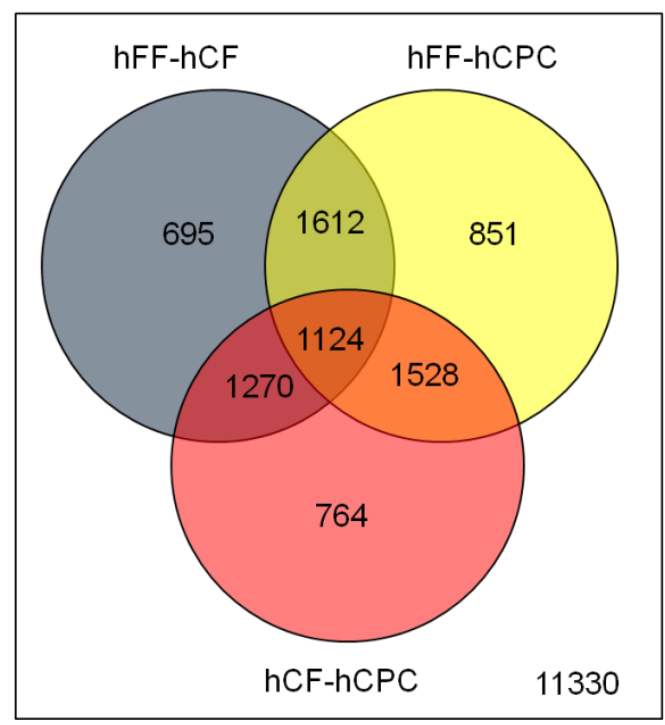

C
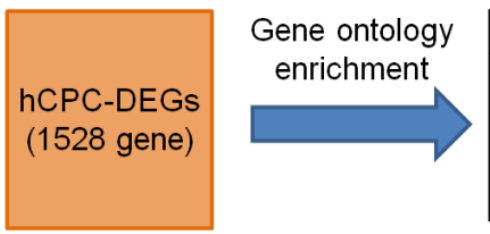

\begin{tabular}{|lc|}
\hline Functional clustering & Enrichment score \\
\hline Plasma membrane & 6,73 \\
Cell adhesion & 6,18 \\
Actin cytoskeleton organization & 4,28 \\
Extracellular region & 3,76 \\
\hline
\end{tabular}

Figure 20. Comparative transcriptome analysis. A Principal component analysis of gene expression in hFFs, hCFs, hCPCs and hCMs ( $n=3 /$ group). B Venn diagram representing the number of differentially expressed genes (DEGs) (Gray: DEGs in hFFs and hCFs, Yellow: DEGs in hFFs and hCPCs, Red: DEGs in hCFs and hCPCs. Intersection of yellow and red circles (in orange) represent DEGs $(1,528)$ specific to hCPCs. C Gene ontology enrichment analysis of hCPC-specific DEGs $(1,528$ genes; represented in orange in the Venn diagram above).

The RNAseq data were further utilized to better define the identity of hCPCs, by analyzing the expression level of genes typically expressed in specific cell populations in the heart: (1) cardiomyocytes, (2) fibroblasts, (3) smooth muscle cells, (4) endothelial cells, (5) pericytes and (6) perivascular MSC-like cells.

hCPCs did not express the mature cardiac marker, TNNI3 and early cardiac marker NKX2-5, but GATA4 in comparison to hCMs, confirming the earlier PCR data (Figure 21A). GATA4 was also detected in hCFs in line with published evidence (Furtado et al. 2014) (Figure 21A). hCPCs showed low levels of endothelial cell marker expression comparable to hCFs such as vascular endothelium calcium dependent 
adhesion molecule (CDH5), CD31 (PECAM1) and kinase insert domain receptor (KDR; Figure 21B). Expression analysis of smooth muscle cell markers further indicated that they express smooth muscle actin (ACTA2), but not mature smooth muscle cell markers like desmin (DES) and myosin heavy chain 11 (MYH11; Figure 21C). They expressed the fibroblast markers transcription factor 21 (TCF21) and periostin (POSTM) at lower levels, but higher level of DDR2 compared to hCFs (Figure 21D). Interestingly, they seemed to be positive for all the markers expressed in pericytes; especially MCAM and chondroitin sulfate proteoglycan (CSPG4) when compared to all other cell types as well as the PDGFR beta subunit (PDGRB; Figure 21E). Lastly, hCPCs also showed markedly higher expression of perivascular mesenchymal stem cell (MSC)-like cells associated marker (Kramann et al. 2015) glioma associated oncogene homolog 1 (GL/1; Figure 21F). 
A

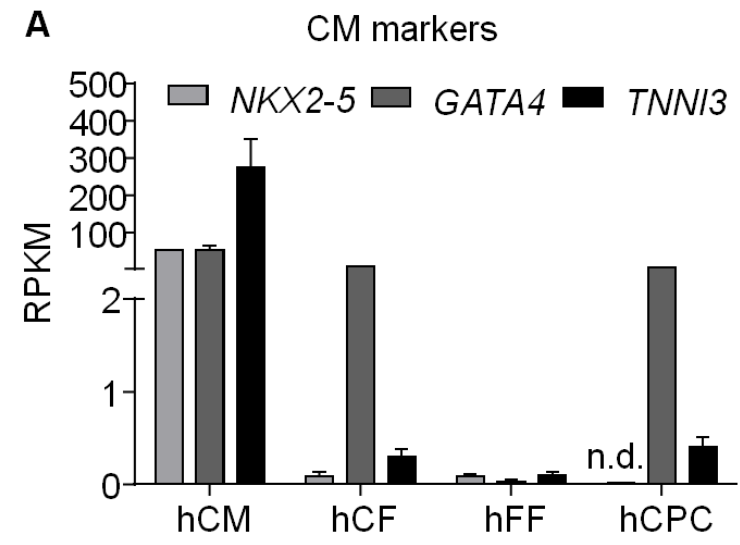

C

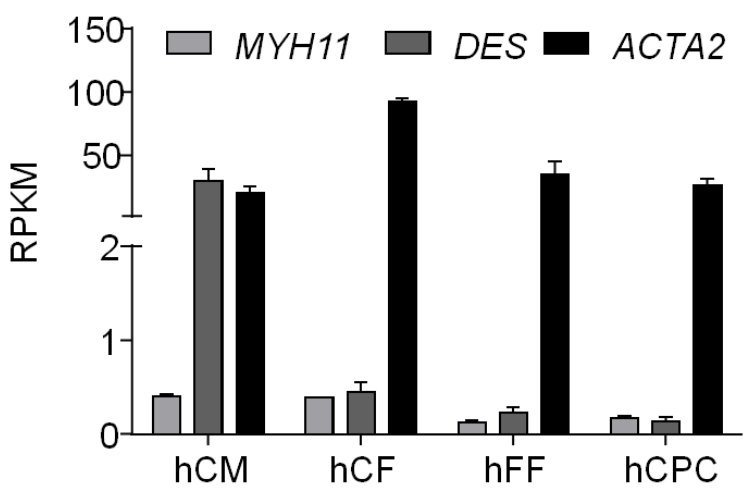

E

Pericyte markers

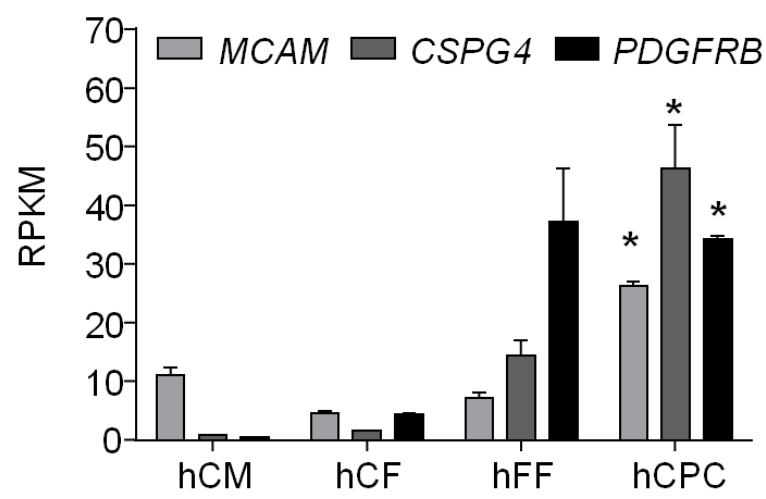

B

EC markers

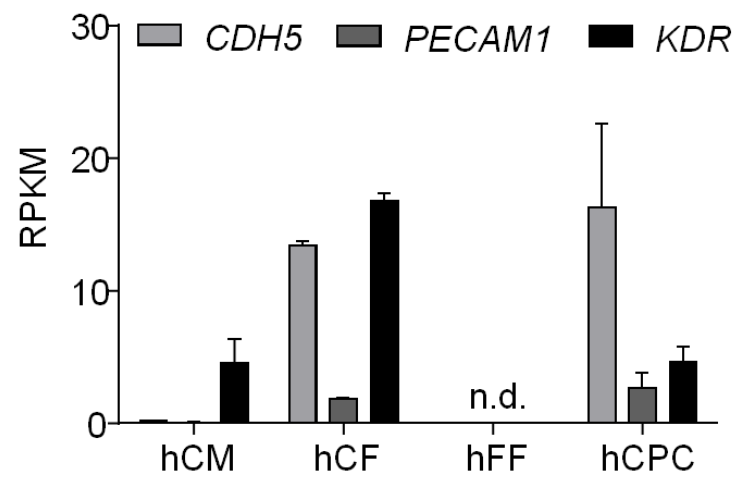

D

Fibroblast markers

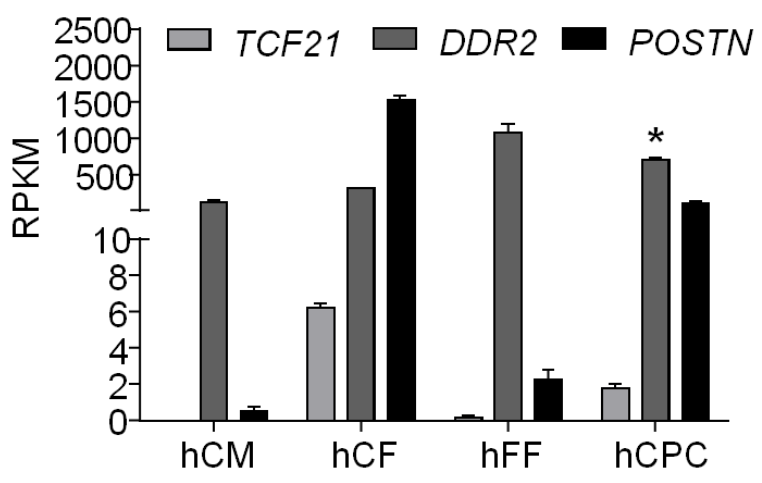

$\mathbf{F}$

Perivascular MSC-like cells

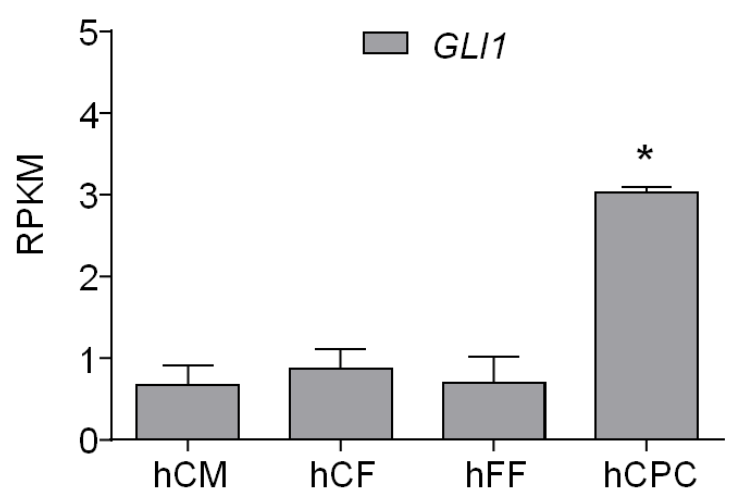

Figure 21. Expression profile of cardiac cell-specific markers. Data is from RNAseq datasets and displayed as RPKM (reads per kilobase of transcript per million mapped reads) values from the individual cell populations (hCMs, hCFs, hFFs and hCPCs; $n=3 /$ group). A Cardiomyocyte (CM) markers NKX2-5, GATA4, and TNNI3; B endothelial cell (EC) markers CDH5, PECAM1 and KDR; C smooth muscle cell (SMC) markers MYH11, DES and ACTA2; D fibroblast markers TCF21, DDR2 and POSTN; E pericyte markers MCAM, CSPG4 and PDGFRB; F perivascular MSC-like cell marker GLI1. ${ }^{*} \mathrm{p}<0.05, \mathrm{hCF}$ vs. hCPC by one-way ANOVA with Dunnett's post-hoc test. 
Taken together, transcriptome profile analyses suggested a pericyte and MSC-like origin of hCPCs with little evidence for cardiomyocyte, smooth muscle, fibroblast or endothelial fate.

\subsection{Cardio-supportive effects of mouse CPCs in EHM}

Stromal cells play an important role in supplying key factors (e.g. extracellular matrix, growth factors, cytokines) for the formation of a cardiogenic niche (Christalla et al. 2012, Tiburcy et al. 2011). Engineered heart muscle (EHM), as a model of heart muscle development in vitro, may be utilized as an experimental platform to gain insight into specific cardio-instructive properties of stromal cells, including CPCs.

\subsubsection{CPCs support functional maturation of EHM}

To investigate if mouse CPCs support cardiac muscle formation, EHMs were made from purified mouse ESC-derived cardiomyocytes (mCMs; $82 \pm 4 \%$ a-actinin positive, $\mathrm{n}=5$; Figure 22A,B) and mouse adult heart derived c-Kit-mCPCs. In a first set of experiments the optimal myocyte/non-myocyte ratio was determined. mCMs and mCPCs were mixed in different ratios (CM/CPC) ranging from $100 / 0 \%$ to $50 / 50 \%$ respectively, keeping the total cell number the same. After 3 days of hydrogel consolidation inside custom-made molds, tissues were transferred onto static stretchers to be mechanically stimulated for functional maturation for the following 11 days (Figure 22C).

Macroscopic analysis of muscle morphology showed that EHMs composed of only cardiomyocytes were distinctly larger and softer than the EHMs supplemented with mCPCs or mouse cardiac fibroblasts ( $\mathrm{mCFs}$ ), supporting the previously identified role of stromal cells (CFs) for tissue compaction. mCPCs supported EHM formation similarly as mCFs (Figure 22D). 
A

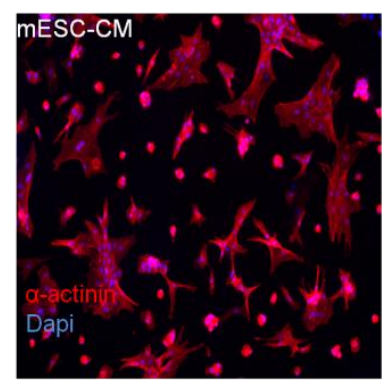

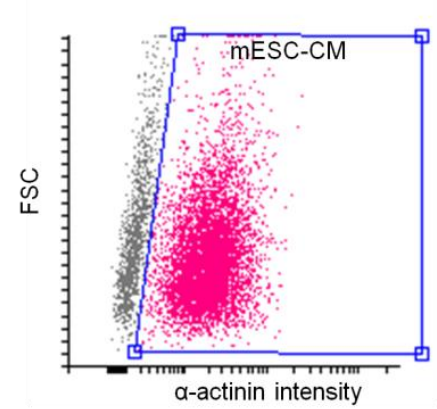

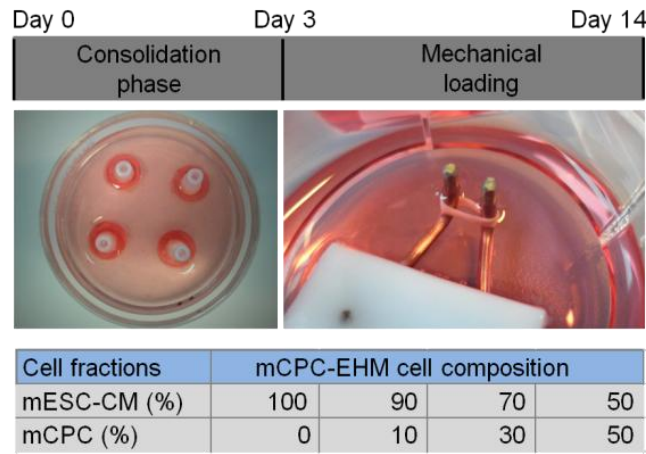

- $0 \% \mathrm{mCPC}$

- $10 \% \mathrm{mCPC}$

$\checkmark \quad 30 \% \mathrm{mCPC}$

- $50 \% \mathrm{mCPC}$

G

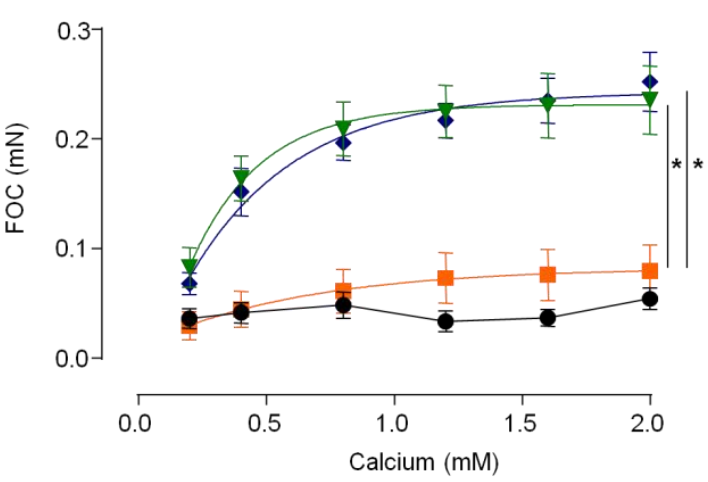

Figure 22. EHM structure and function enhanced by mCPCs. A Cardiomyocytes dissociated from antibiotic-selected mouse cardiac bodies after spontaneous cardiac differentiation (red: $\alpha$-actinin, blue: Hoechst nuclear staining) and B flow cytometry analysis after staining for sarcomeric a-actinin to determine cardiomyocyte purity $(n=5)$. C EHM cell composition: cardiomyocytes (mESC-CMs) and mCPCs ratio were as indicated in the box; the culture as indicated for 3 days in custom-made molds for tissue consolidation, followed by 11 days of mechanical stimulation for functional maturation. D GFP fluorescence (mCPCs were GFP ${ }^{+}$) and brightfield images (top right) of EHM without CPCs $(0 \%)$, $30 \% \mathrm{mCFs}$ and $30 \% \mathrm{GFP}^{+} \mathrm{mCPCs}$. E Cardiomyocyte structure and alignment in mEHM with $0 \% \mathrm{GFP}^{+}$ mCPCs and $\mathbf{F} 30 \%$ GFP $^{+}$mCPCs (indicated by arrows). G Force of contraction (FOC) generated in $m E H M$ in response to increasing extracellular calcium concentration $(n=7 /$ group from two experiments, two-way ANOVA, $\left.{ }^{*} p<0.05\right)$.

Immunostaining of EHMs without CPCs (CM/CPC; 100/0\%) revealed that cardiomyocytes (red, $\alpha$-actinin positive) remained round shaped in small clusters and distributed randomly without proper alignment and coupling throughout the matrix 
(Figure 22E). Consistent with the underdeveloped structure of the cardiomyocytes, EHMs without mCPCs failed to develop any measurable force (Figure 22G). In contrast, EHM composed of cardiomyocytes supplemented with mCPCs showed enhanced alignment and sarcomere development. Accordingly, force of contraction (FOC) was markedly enhanced in EHM supplemented with $>10 \%$ mCPC (Figure 22F,G).

We next wanted to scrutinize potential differential effects of mCPCs and fibroblasts on functional maturation of EHM. For this, a supplementation of mCMs with $30 \%$ mCPCs or mouse embryonic fibroblasts (mEFs) was performed. Indeed, EHMs generated with mCPCs produced FOC similar to the EHMs supplemented with $\mathrm{mEFs}$, suggesting that $\mathrm{mCPCs}$ have comparable cardio-instructive properties as fibroblasts (mEF-EHM: $0.36 \pm 0.05 \mathrm{mN}$, mCPC-EHM: $0.29 \pm 0.04$ at $3.2 \mathrm{mM}$ calcium, $\mathrm{n}=16$ /group; Figure 23A). Interestingly, mCPC-EHM displayed markedly higher sensitivity to calcium, suggesting for a more immature calcium handling machinery when compared to mEF-EHM (mEF-EHM: $0.74 \pm 0.05$ mM EC 50 calcium, mCPC-EHM: $0.53 \pm 0.22 \mathrm{mM} \mathrm{EC}_{50}$ calcium; $p<0.05, n=16 /$ group Figure 23B).
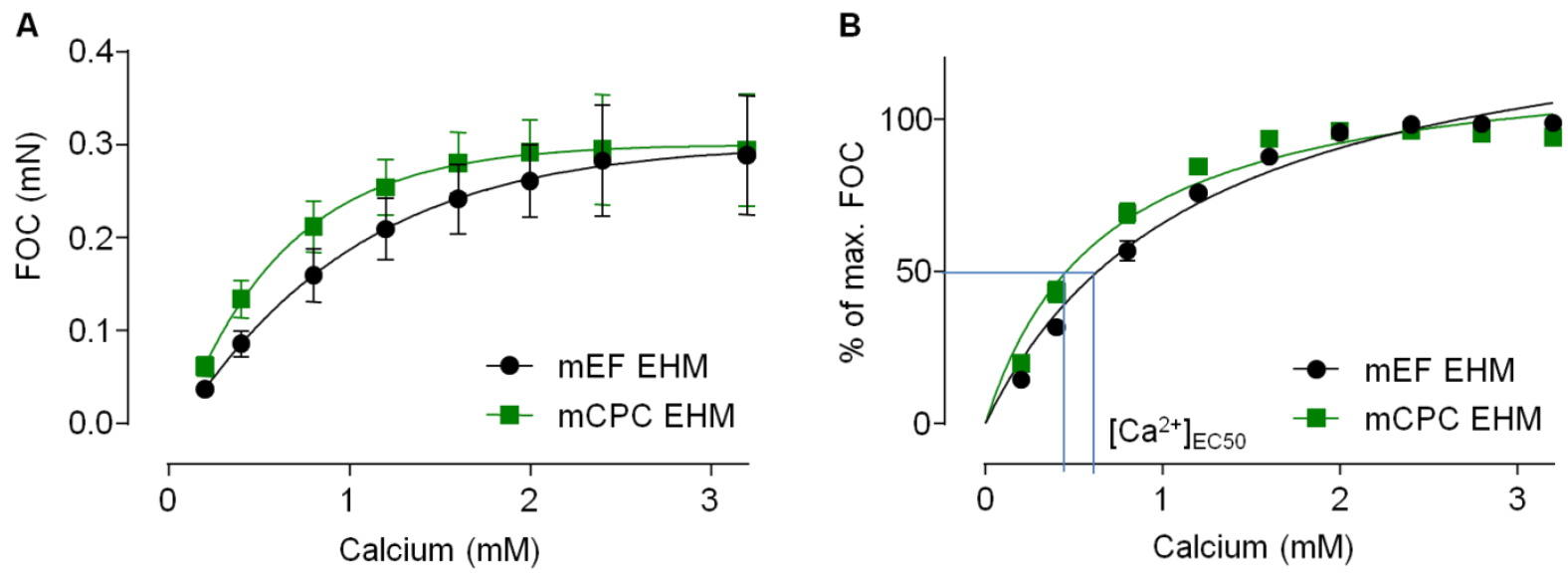

Figure 23. Functional comparison of mCPC- and mEF-EHMs. A Force of contraction (FOC) generated in mCPC- and mEF-EHM under increasing extracellular calcium concentration in thermostatted $\left(37^{\circ} \mathrm{C}\right)$ Tyrode's solution and electrical field stimulation $(2 \mathrm{~Hz} ; \mathrm{n}=16 / \mathrm{group})$. B Display of FOC as percent of maximal FOC to determine the $\mathrm{EC}_{50}$ for calcium in $\mathrm{mEF}-\mathrm{EHM}$ and $\mathrm{mCPC}$-EHM; the $E C_{50}$ for the respective groups is marked by blue lines ( $n=16 /$ group, unpaired $t$-test, ${ }^{*} p<0.05$ ). 


\subsection{Enhanced paracrine support by genetic manipulation of mouse CPCs}

Paracrine factors are one of the key components contributing to the cardiogenic niche (Christalla et al. 2012). The paracrine activity of mCPCs may support cardiac function and could be augmented by overexpression of microRNA-133a. MicroRNA $(\mathrm{miR})-133 \mathrm{a}$ is one of the muscle specific microRNAs that play an important role in the regulation of embryonic heart development and cell death (Meder et al. 2008).

Further, it was observed that mCPCs overexpressing miR-133a enhance cardiac functions upon injection into the heart after myocardial infarction in a rat model (Izarra et al. 2014). Reduced cardiomyocyte death and hypertrophy as well as increase in the number of DNA-replicating cardiomyocytes were suggested as the possible mechanisms underlying the beneficial effect in vivo, which could have been mediated by enhanced paracrine activity in CPCs overexpressing miR-133a. To further scrutinize this hypothesis, we developed an EHM model with defined neonatal rat cardiomyocytes and $\mathrm{mCPC}$ composition (3:1 ratio). mCPCs were genetically modified to express miR-control or miR-133a. Purity of neonatal rat cardiomyocytes was enhanced by preplating ( $84 \pm 3 \%$ a-actinin positive, $n=8)$, the remaining $\sim 15 \%$ percent are mainly comprised of rat firbroblasts (Figure 24). mCPC supplemented EHM could be easily distinguished by the expression of GFP (Figure 25A). 


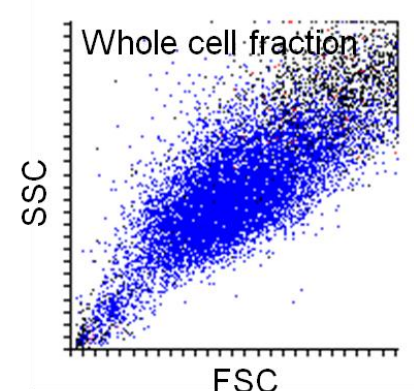

FSC

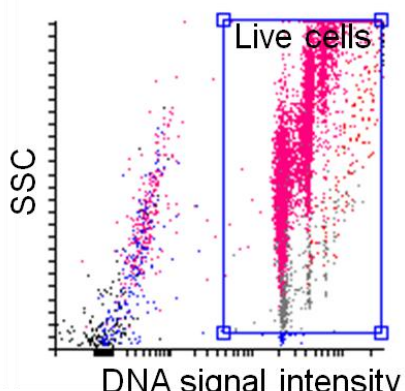

DNA signal intensity

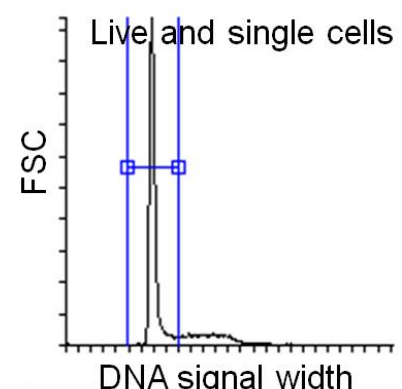

DNA signal width
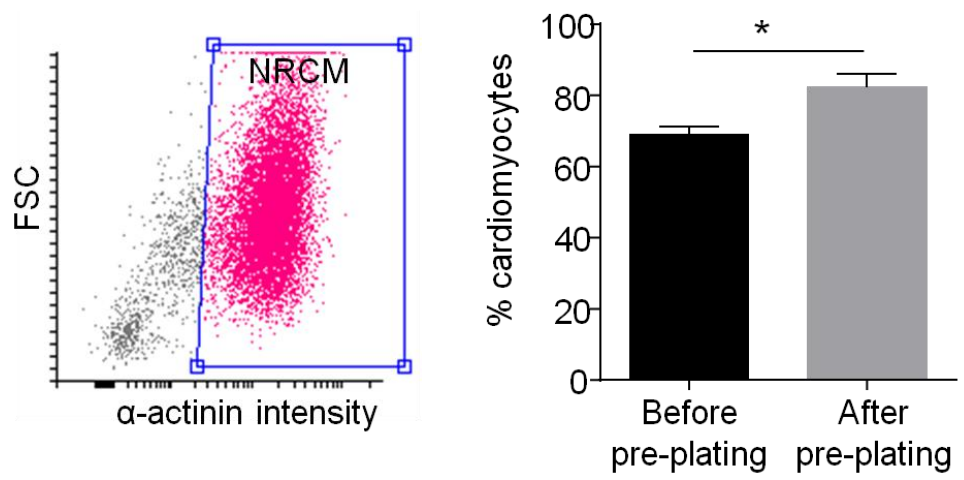

Figure 24. Purification of neonatal rat cardiomyocytes. Gating strategy of the flow cytometry analysis of cardiomyocyte purity is displayed. Forward (FSC) and sideward scatter (SSC) analyses were followed by gating living cells based on DNA signal intensity and signal width (Hoechst-nuclear staining). Cardiomyocytes were identified by specific labelling for $\alpha$-actinin positive before and after myocyte enrichment by pre-plating ( $n=8 /$ group from 8 experiments, unpaired $t$-test, ${ }^{*} p<0.05$ ).

EHMs with miR-133a-CPCs developed significantly higher FOC (Figure 25B). The number of apoptotic cardiomyocytes (caspase-3 positive) was reduced at day 3 , which resulted in a higher percentage of cardiomyocytes remaining in miR-133aEHM at day 12 when compared to miR-control-EHM (Figure 25C,D). Real-time PCR analysis of growth factor expression in mCPCs revealed that expression level of Igf1, Vegf, Hgf and bFgf, which are involved in cardiomyocyte survival, growth and proliferation, were all increased in miR-133a-EHM, suggesting an involvement of miR-133a in the paracrine activity of mCPCs (Figure 25E). Collectivley, this data supported the in vivo studies in providing addition evidence for genetically enhanced paracrine activity of mCPCs by miR-133a. 
A

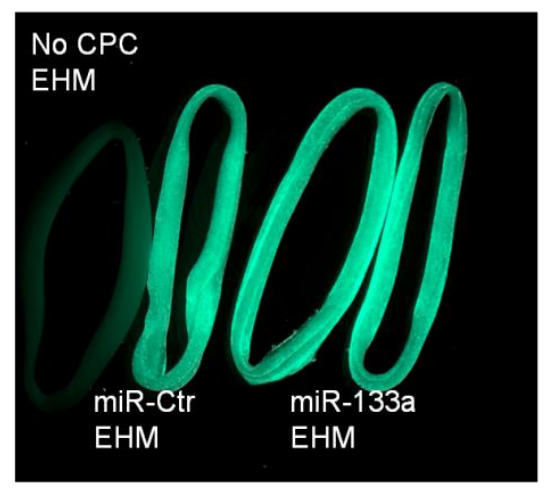

C

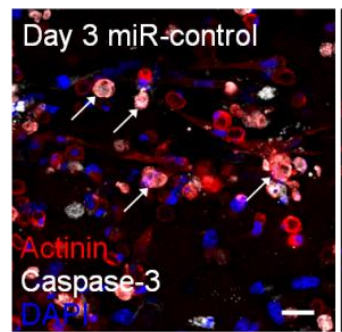

D

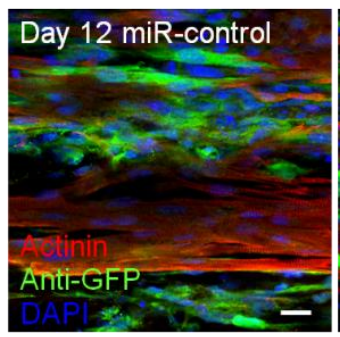

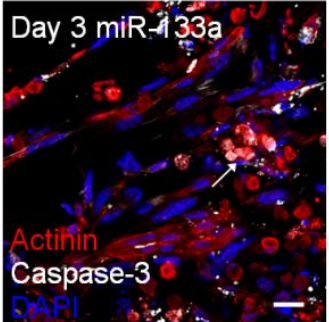

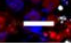

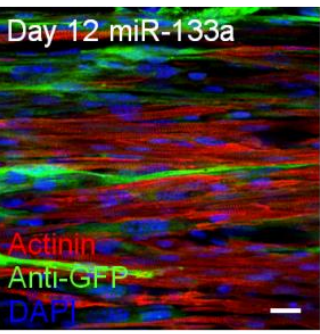

B
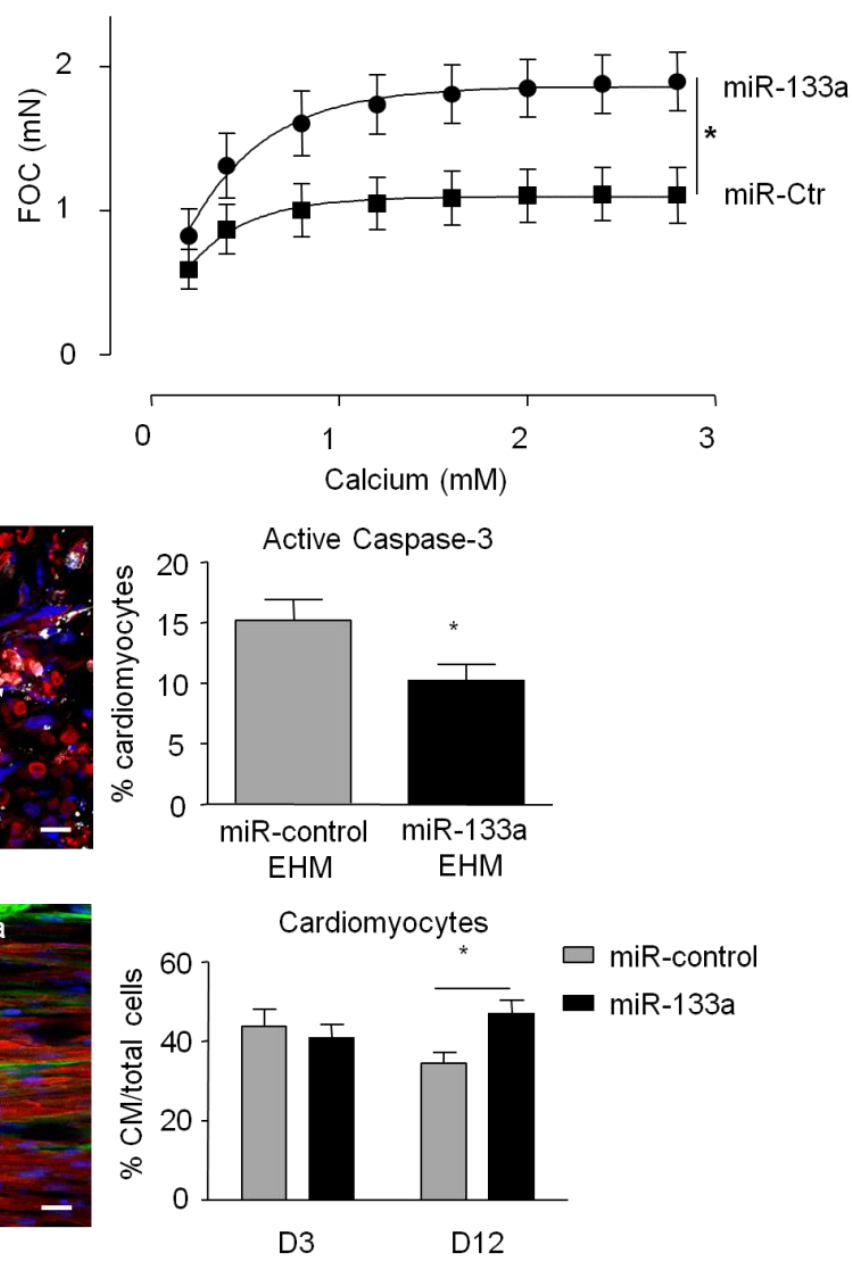

E

bFGF

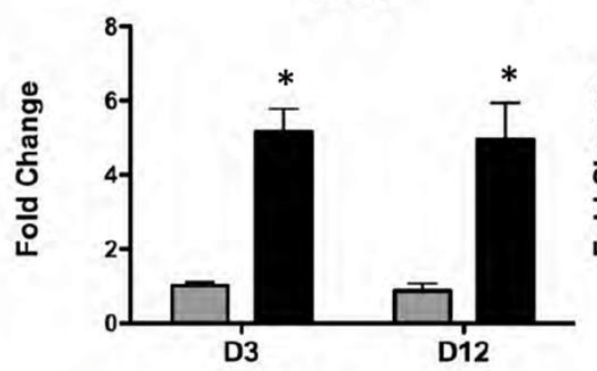

HGF
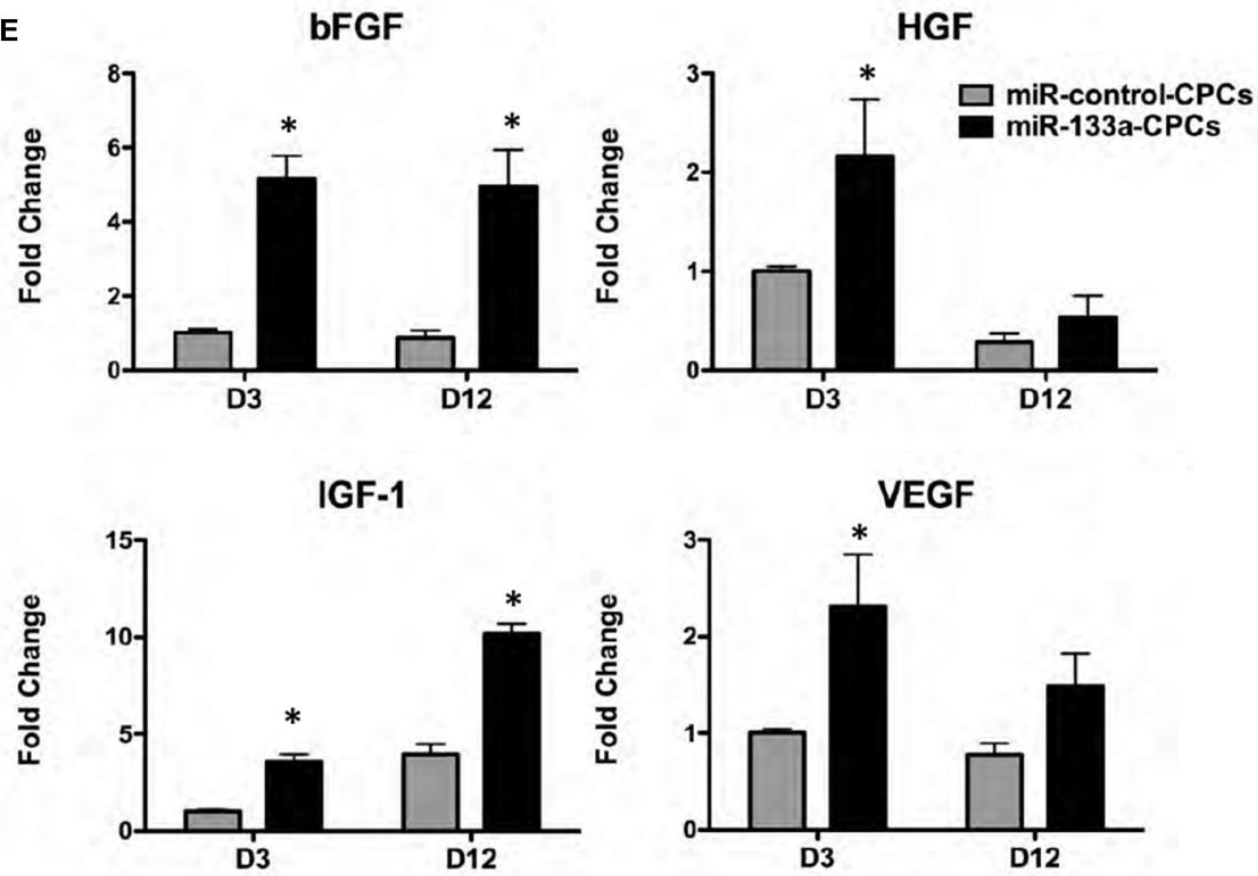
Figure 25. miR-133a enhanced paracrine activity of mCPCs. A EHM containing mCPC could be identified by $\mathrm{GFP}^{+}$fluorescence; images were taken on culture day 12. B Force of contraction (FOC) generated in miR-control $(n=6)$ and miR-133a-CPC-EHMs $(n=6)$ in response to calcium. C Representative images of whole-mount caspase- 3 staining in miR-control- and miR-133a-CPC-EHMs (left; $\alpha$-actinin, red; activated caspase-3, white; DAPI-stained nuclei, blue; scale bars: $20 \mu \mathrm{m}$ ); bar graph (right): quantification of the percentage of caspase-3 positive cardiomyocytes in EHM at culture day $3 ; n>1,000$ cells from three experiments. D Representative images of cardiomyocytes with miRcontrol- and miR-133a-CPCs (left; $\alpha$-actinin, red; antibody detected GFP, green; DAPI-stained nuclei, blue; scale bar: $20 \mu \mathrm{m}$ ); bar graph (right): quantification of the percentage of cardiomyocytes in EHM at culture days 3 and 12; $\mathrm{n}>1,000$ /group from two and three experiments. E Expression level of $b F g f$, Hgf, lgf-1 and Vegf in miR-control- and miR-133a-CPC-EHMs at culture day 3 and 12; PCR was performed by Alberto Izarra ( $n=3$ /group from three experiments). ${ }^{*} p<0.05$ by two-way ANOVA with Bonferroni post-hoc test (B) and unpaired, two-tailed Student's $t$-test (C-E). Data was published in (Izarra et al. 2014).

\subsection{Human EHM model}

To investigate possible cardiogenic function of human CPCs (hCPCs), hEHMs using the same principles as in the mouse model were generated. Human cardiomyocytes were derived from human embryonic stem cells (HES2-RFP) after directed differentiation and metabolic selection (average cardiomyocyte purity: $89 \pm 3 \% \alpha$ actinin $^{+}$cells; $n=3$, Figure 26A). In parallel, $h E H M s$ were made with fibroblasts derived from human foreskin (hFFs) to serve as a reference (Figure 26B). 


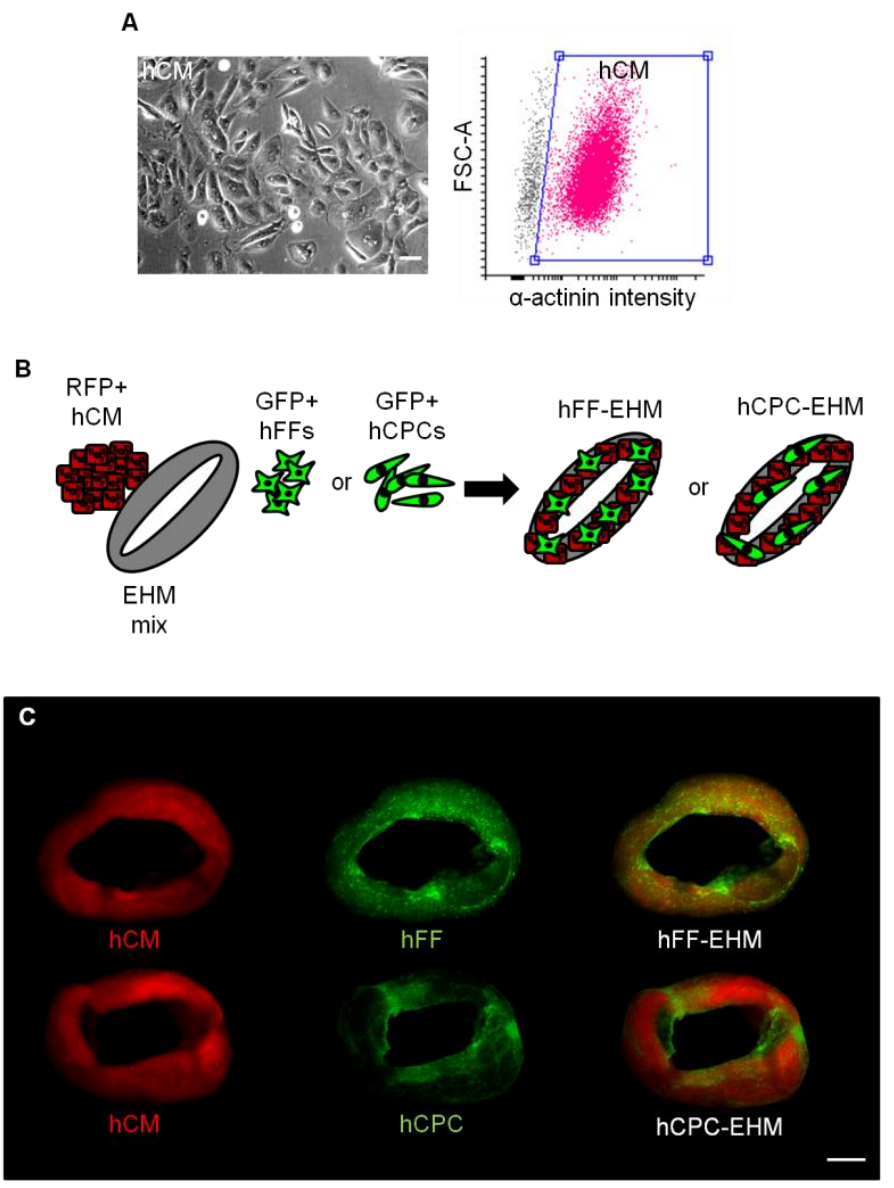

Figure 26. Defined hEHM model composed of $\mathrm{RFP}^{+}$-hCMs and $\mathrm{GFP}^{+}$-non-myocytes. A Brightfield image of hCMs with a representative flow cytometry plot (right) with the identification of cardiomyocytes (sarcomeric $\alpha$-actinin ${ }^{+}$). B Schematic representation of hEHM construction strategy with cell-type color-coding: hCMs RFP ${ }^{+} / \mathrm{hFFs} \mathrm{GFP}^{+} /$hCPCs GFP . C hFF-EHM (RFP ${ }^{+}$hCMs, GFP $^{+}$ hFFs) and hCPC-EHM (RFP $\left.{ }^{+} \mathrm{hCMs}, \mathrm{GFP}^{+} \mathrm{hCPCs}\right)$ under GFP and RFP fluorescence, with merged images (rightmost). Scale bar: $1 \mathrm{~mm}$.

Based on the fluorescence imaging of RFP and GFP positive cells in EHM sections, cardiomyocytes and fibroblasts were observed more randomly distributed within the hFF-EHM matrix, whereas hCPCs tended to cluster towards the surface of the hCPC-EHM (Figure 27A). This finding suggests that hCPCs have the property to migrate in EHM. Cardiomyocytes showed similar elongation and cross-striations in both hFF- and hCPC-EHMs (Figure 27B). 
A

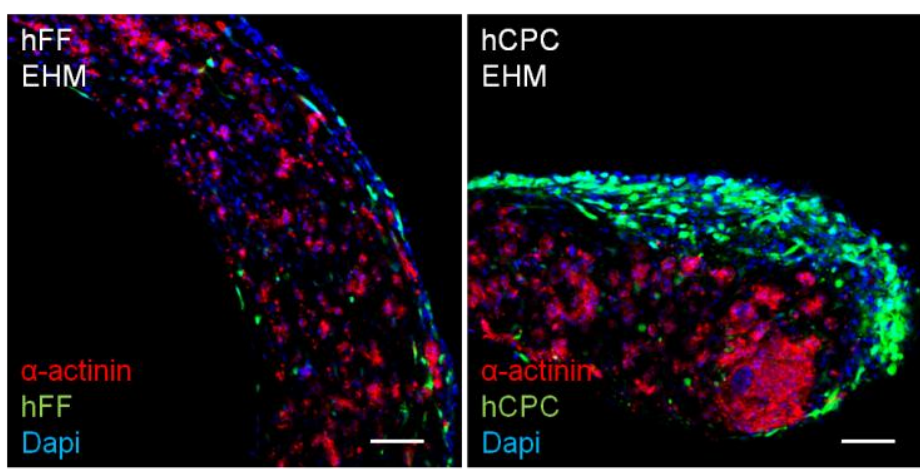

B
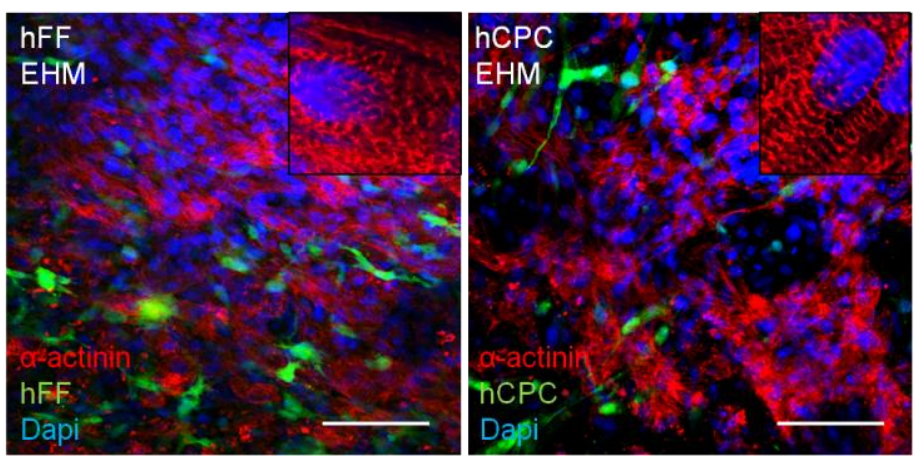

Figure 27. Cell distribution and cardiomyocyte morphology in hEHM. A Low magnification overview of $\mathrm{RFP}^{+}$hCMs and GFP ${ }^{+}$non-myocytes in hFF-EHM (left) and hCPC-EHM (right). B Orientation and structure of cardiomyocytes in hFF-EHM (left) and hCPC-EHM (right). (Cardiomyocytes: red, a-actinin ${ }^{+}$; hFFs and hCPCs: GFP ${ }^{+}$; nuclei stained with the DNA-binding dye Hoechst in blue). Scale bars: $100 \mu \mathrm{m}$.

\subsubsection{Contractile performance in human EHM}

hFF-EHM developed significantly higher FOC than hCPC-EHM (hFF-EHM: 0.67 \pm 0.08 $\mathrm{mN}, \mathrm{hCPC}$-EHM: $0.43 \pm 0.03 \mathrm{mN}$ at $4 \mathrm{mM}$ calcium, $\mathrm{n}=29$ /group ${ }^{*} \mathrm{p}<0.05$; Figure 28A). On the other hand, they showed similar sensitivity to calcium (hFF-EHM: $0.88 \pm 0.26$ $\mathrm{mM} \mathrm{EC}_{50}$ calcium, hCPC-EHM: $0.97 \pm 0.37 \mathrm{mM} \mathrm{EC}_{50}$ calcium, $\mathrm{n=29/group;} \mathrm{Figure}$ 28B) suggesting similar maturation of calcium handling machinery. $\beta$-adrenergic stimulation with isoprenaline $(1 \mu \mathrm{mol} / \mathrm{L})$ significantly enhanced $\mathrm{FOC}$ development in both $\mathrm{hEHMs}$ at $\mathrm{EC}_{50}$ calcium concentration (increase in FOC from baseline: $91 \pm 14 \%$ in hFF-EHM, $61 \pm 9 \%$ in hCPC-EHM; $n=29$ /group), with however no obvious difference between the investigated groups (Figure $\mathbf{2 8 C}$ ). Both hEHM co-cultures responded to cholinergic stimulation (carbachol) with the anticipated decrease in FOC, with however a significantly smaller reduction in FOC in hCPC-EHMs as compared to 
hFF-EHM (decrease in FOC: hFF-EHM: 17 $\pm 2 \%$, hCPC-EHM: 12 $\pm 2 \%, n=29 /$ group, ${ }^{*} \mathrm{p}<0.05$; Figure 28C).

A
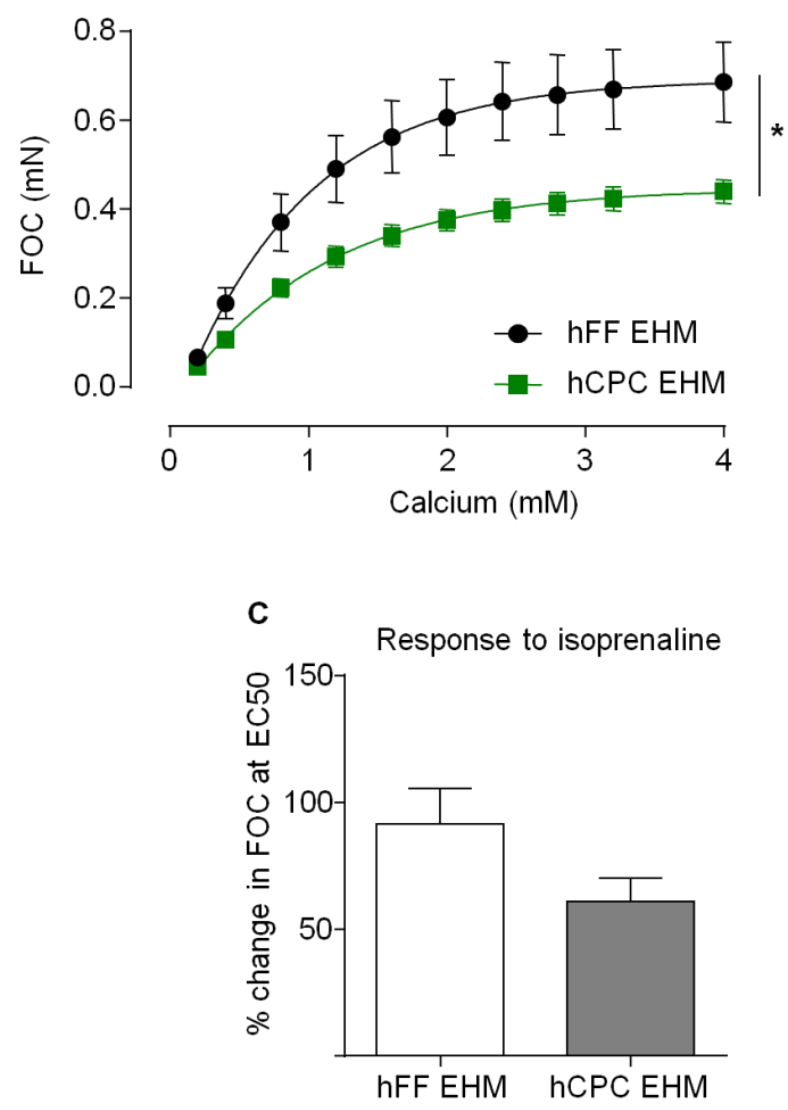

B
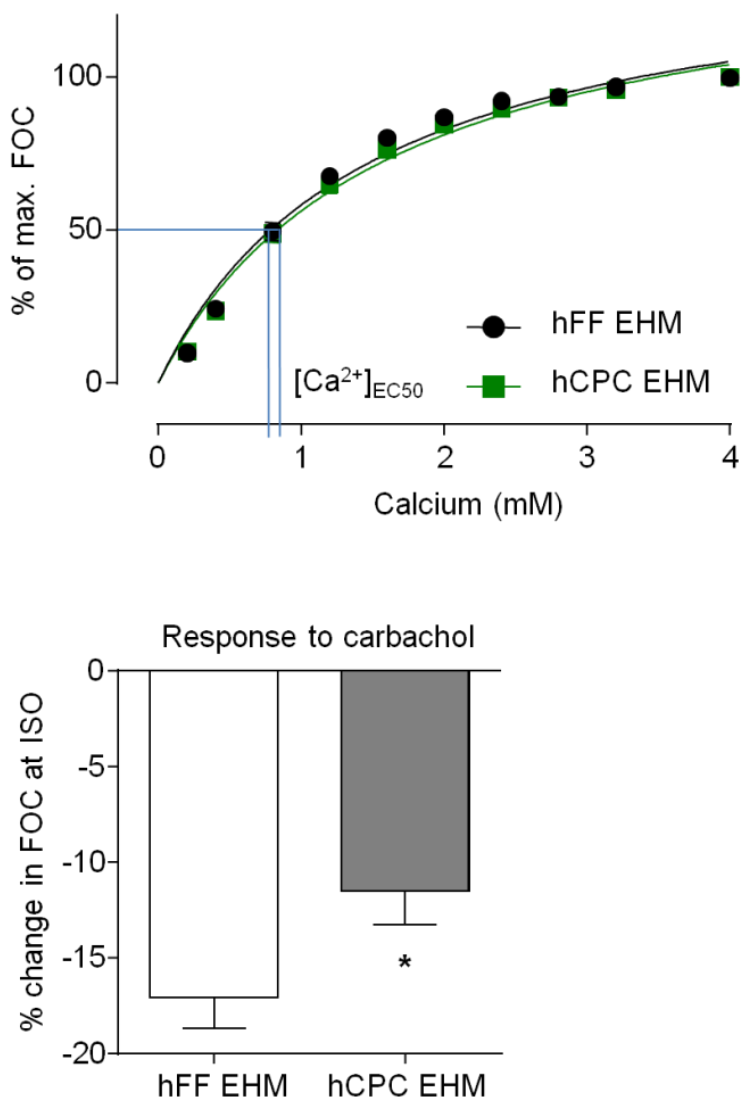

Figure 28. hCPCs and hFFs supported EHM. A Force of contraction (FOC) generated in hFF- and hCPC-EHM in response to gradually increasing extracellular calcium concentrations. B FOC as percent of maximal FOC to determine the apparent $\mathrm{EC}_{50}$ for calcium in hFF- and hCPC-EHM; the $\mathrm{EC}_{50}$ for the respective groups is marked by blue lines ( $n=29 /$ group). C Response to isoprenaline $(1 \mu \mathrm{mol} / \mathrm{L}$ ) as percentage change in $\mathrm{FOC}$ at $\mathrm{EC}_{50}$ calcium and response to carbachol $(10 \mu \mathrm{mol} / \mathrm{L})$ as percentage change in FOC after treatment with isoprenaline ( $n=29 /$ group, unpaired $t$-test, $\left.{ }^{*} p<0.05\right)$.

\subsubsection{Passive biomechanical properties in human EHM}

Besides the role of providing structural support and organization to the tissue, the biophysical properties of EHM may be affected by stromal cells. Stromal cells are key contributors to EHM stiffness (Naito et al. 2006). To identify whether hFFs and hCPCs influence tissue stiffness differentially, resting tension (ReT - diastolic force) was determined and found to be similar in hFF-EHM and hCPC-EHM (Figure 29A). 
Accordingly, FOC/ReT ratio was higher in hFF-EHM as compared to hCPC-EHM (2.5 \pm 0.3 vs. $1.5 \pm 0.2, n=28 /$ group, unpaired t-test, ${ }^{*} p<0.05$; Figure 29B).
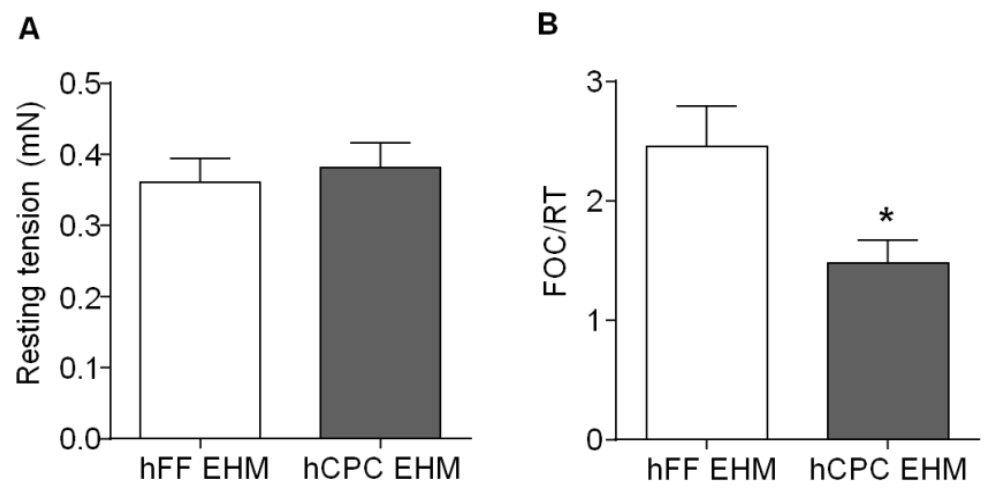

Figure 29. Passive mechanical properties of hEHM. A Resting tension (ReT) at Lmax and $B$ ratio of force of contraction (FOC) to ReT in hFF- and hCPC-EHMs ( $n=28$ /group, unpaired $t$-test, ${ }^{*} p<0.05$ ). Lmax: length at maximum FOC.

Taken together, histological and functional analysis of hEHMs suggested that hCPCs were able to support heart muscle formation in EHM, albeit less efficiently as compared to hFFs. Differences in hFF versus hCPC localization, i.e., homogeneous versus surface localization will require further investigations.

\subsubsection{Cardiomyocyte structure and function in human EHM}

To further investigate the underlying mechanisms leading to less functional development in hCPC-EHM, cardiomyocyte content, maturation and structural development were assessed in comparison to hFF-EHM.

Cardiomyocyte amount in EHM was assessed through quantification of sarcomeric $\alpha$ actinin positive cells by flow cytometry. The number of cardiomyocytes recovered from EHM after 14 days of culture was similar between the groups (hFF- vs. hCPCEHM: $3 \pm 0.3 \times 10^{5}$ vs. $3.2 \pm 0.2 \times 10^{5}, n=10$ /group from three experiments; Figure 30A) suggesting that the actual reason of lower FOC development in hCPC-EHM was not due to the muscle cell amount, but less FOC generation per cardiomyocyte (hFF- vs. hCPC-EHM: $3 \pm 0.4$ vs. $1.6 \pm 0.1 \mathrm{nN}, \mathrm{n}=3$ /group, from three experiments, ${ }^{*} p<0.05$; Figure 30B). To investigate the possible reason behind this impaired functional 
maturation of cardiomyocytes in hCPC-EHM, the percentage of cardiomyocytes expressing Ki67 as a marker for maintained cell cycle activity in cardiomyocytes was analyzed. However, no significant difference was detected between the EHM coculture groups (hFF- vs. hCPC-EHM: $19 \pm 5$ vs. $17 \pm 4 \%, n>1,000$ cells/group from three experiments; Figure 30C). Next, cardiomyocyte size and actinin intensity were compared to assess their structural maturation in the EHMs by flow cytometry analysis. Cardiomyocytes were not different in size $(n=10 /$ group from three experiments; Figure 30D), but demonstrated almost $10 \%$ less sarcomeric $\alpha$-actinin in hCPC-EHM compared to the cardiomyocytes in hFF-EHM (actinin signal intensity/cardiomyocyte relative to hFF-EHM group: $1 \pm 0.03$ vs.0.91 \pm 0.04 in hCPCEHM, $n=10$ /group from three experiments, ${ }^{*} p<0.05$ (Figure 30E). 

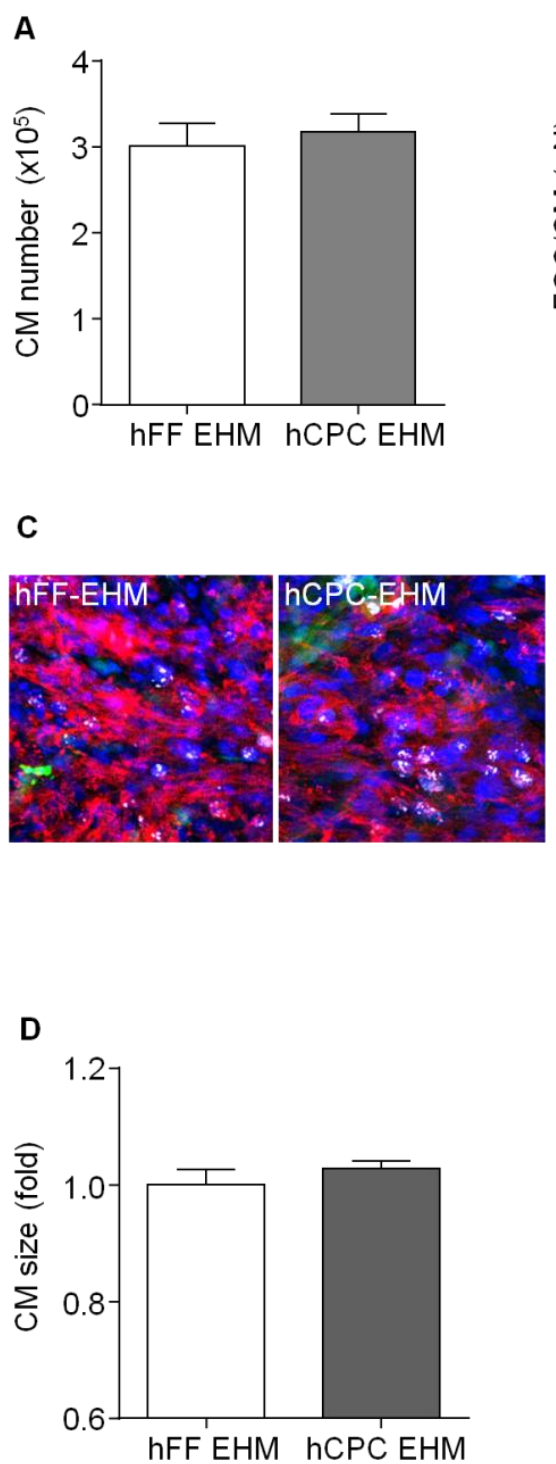

B
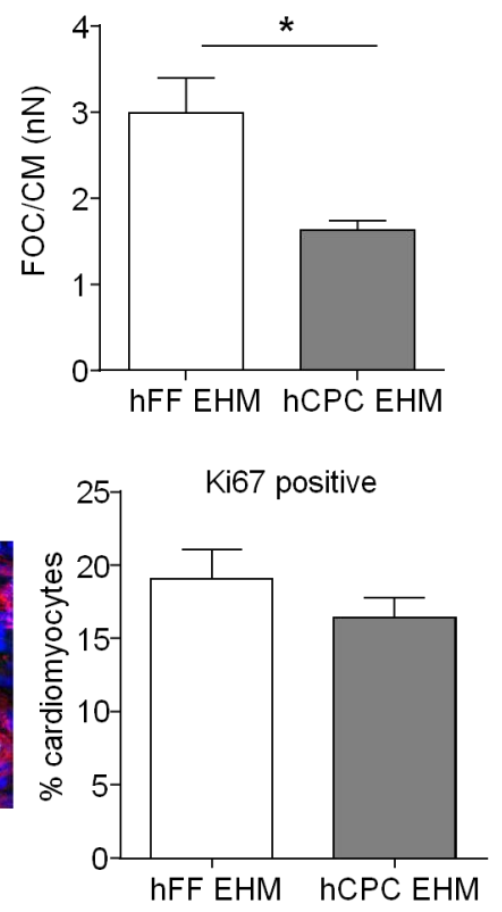

E

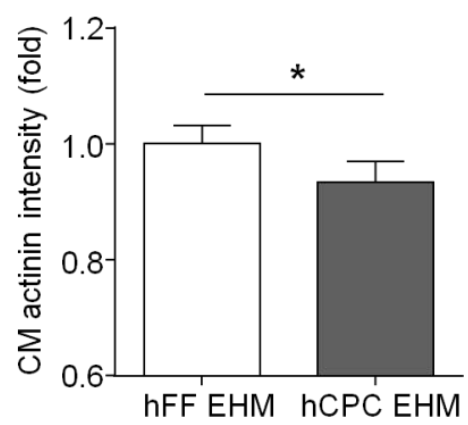

Figure 30. Cardiomyocyte amount and phenotype in hEHM. A Number of cardiomyocytes ( $\alpha$ actinin positive cells detected by flow cytometry) in hFF- and hCPC-EHM ( $n=10 /$ group). B Force of contraction (FOC) per EHM-cardiomyocyte (maximum FOC at $4 \mathrm{mM}$ of calcium concentration divided by total cardiomyocyte number in EHM; $\mathrm{n}=3$ /group, unpaired $t$-test, $\left.{ }^{*} \mathrm{p}<0.05\right)$. C Representative images of whole-mount Ki67 stainings in hFF- and hCPC-EHM (left; red: $\alpha$-actinin, white: Ki67 and blue: Hoechst stained nuclei); bar graph (right) with quantification of percentage of Ki67 positive cardiomyocytes at day 14, n>1,000 cells from three experiments. D Relative size of cardiomyocytes (FSC-A median of $\alpha$-actinin positive cells by flow cytometry; $n=10 /$ group from three experiments; reference group: hFF-EHM). E Relative $\alpha$-actinin intensity (median fluorescence intensity of $\alpha$-actinin positive cells by flow cytometry, $n=10 /$ group from three experiments; reference group: hFF-EHM; unpaired $t$-test, $\left.{ }^{*} \mathrm{p}<0.05\right)$. 
Taken together, these findings suggest that the potential mechanism behind reduced contractile function in hCPC-EHM when compared to hFF-EHM could be suboptimal structural and functional maturation of cardiomyocytes.

\subsubsection{Human CPC retention in EHM}

Cell retention is generally low after intramyocardial injection (Tossios et al. 2008). Retention of hCPCs in EHM was determined by flow cytometry. Upon dissociation of the EHM into single cell suspension living cells were identified by flow cytometry based on the Sytox DNA binding dye exclusion; single cells were identified by Hoechst staining (Figure 31A). Next, color-coded cell fractions were separated based on their respective fluorescence signals (cardiomyocytes: RFP ${ }^{+}$, stromal cells: GFP $^{+}$); potential cell fusion events should be revealed as double positive cells for RFP and GFP (Figure 31B). A marked reduction on $\mathrm{GFP}^{+}$( $\mathrm{hCPC}$ or $\mathrm{hFF}$ ) cell number was observed by hEHM culture day 14 (day 0: $3.6 \times 10^{5} \mathrm{GFP}^{+}$cells, hFF-and hCPC-EHM on culture day $14: 0.48 \pm 0.04 \times 10^{5}$ and $0.36 \pm 0.06 \times 10^{5} \mathrm{GFP}^{+}$cells, respectively; $n=14 /$ group from three experiments). Double positive cells were rarely detected with $0.7 \pm 0.2$ and $0.3 \pm 0.05 \%$ in hFF- and hCPC-EHM; Figure 31B). 
A
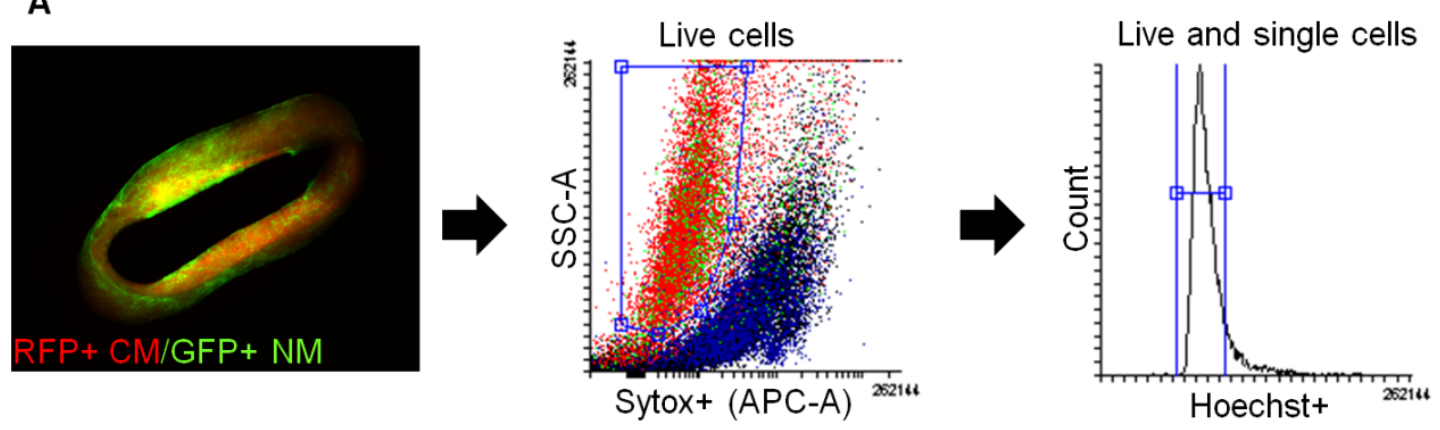

(Pacific Blue-W)

B
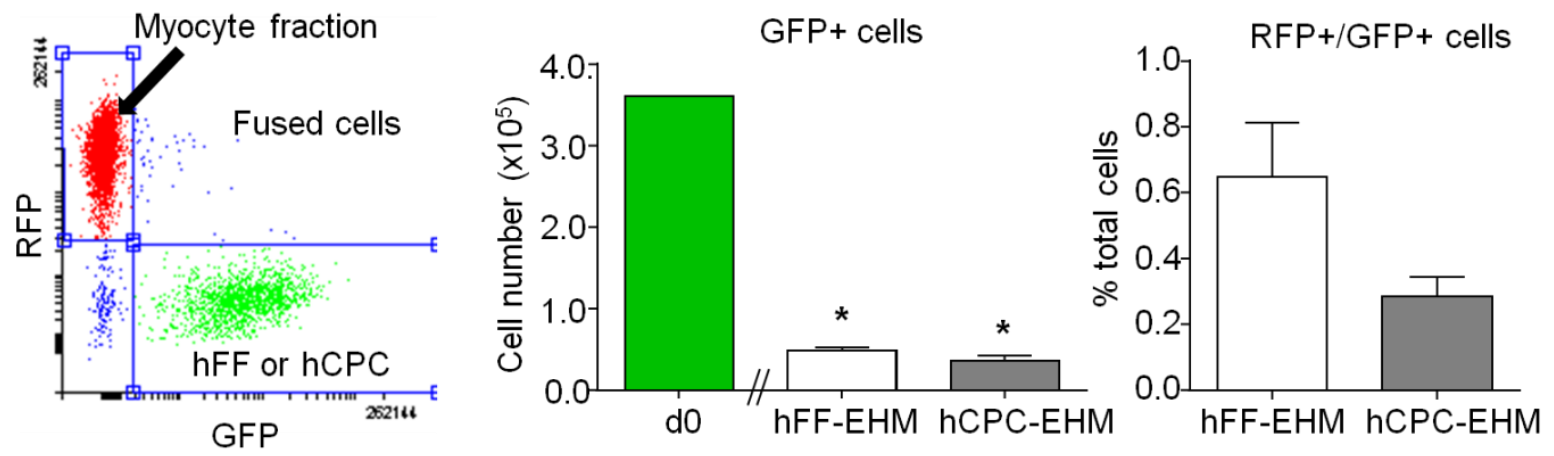

Figure 31. Retention of non-myocytes in hEHM. A Flow cytometry gating strategy to determine living (Sytox negative), single cells (Hoechst positive, with uniform signal width) in color-coded hEHM (cardiomyocytes: $\mathrm{RFP}^{+}$; non-myocytes: $\mathrm{GFP}^{+}$; representative image on the left). B Left plot: flow cytometry gating protocol to separate $\mathrm{RFP}^{+}$cardiomyocytes and or $\mathrm{GFP}^{+}$non-myocytes (NMs: hFFs or hCPCs); middle panel: quantification of the number of $\mathrm{GFP}^{+}$hFFs or GFP ${ }^{+}$hCPCs at day 0 (input number) in comparison to the respectively isolated hFFs and hCPCs from EHM on culture day 14 ( $n=14$ /group); right panel: quantification of the percentage of double positive cells for RFP and GFP (RFP $/$ GFP $^{+}$cells; $n=14$ /group); ${ }^{*} P<0.05$ vs. $d 0$ by Student's $t$-test.

\subsubsection{No evidence of cardiomyocyte transdifferentiation in human EHM}

To investigate whether hCPCs are able to differentiate into cardiomyocytes, EHM were digested into single cells and subjected to the following analyses: (1) flow cytometry analysis of $\alpha$-actinin expressing $\mathrm{GFP}^{+}$cells (Figure 32A), (2) fluorescenceactivated cell sorting (FACS) followed by qPCR for cardiac musle $\alpha$-actin (ACTC1) transcipts in the $\mathrm{RFP}^{+}$cardiomyocyte and $\mathrm{GFP}^{+}$stroma cell pools (Figure 32B).

The cardiomyocyte $\left(\right.$ actinin $^{+}$) and non-myocyte populations could be clearly separated by flow cytometry analysis with a small amount of double positive cells, 
suggesting cell fusion or transdifferentiation in EHM culture; interestingly, there was no difference in the amount of actinin ${ }^{+} / \mathrm{GFP}^{+}$cells in the hFF- and hCPC-EHM (hFFvs. hCPC-EHM: $4.0 \pm 0.7 \%$ vs. $7.4 \pm 1.8 \%, \mathrm{n}=6$ /group from four experiments; Figure 32A). Given no evidence for transdifferentiation of hFFs, we concluded that the similarity of the amount of double positive cell populations in hFF- and hCPC-EHM was most likely due to false-positive antibody labelling in the non-myocyte pool. To further scrutinize this, $\mathrm{RFP}^{+}$cardiomyocytes and $\mathrm{GFP}^{+}$non-myocytes from culture day 14 hEHM were separated by FACS and independently subjected to RNA isolation followed by qPCR to analyze transcription of the cardiomyocyte specific ACTC1 gene. The data from this experiment indicated a 100-fold higher ACTC1 abundance in the hCM pool in comparison to the respective hFF and hCPC pools (Figure 32B). Interestingly, ACTC1 transcript abundance was also 100-fold higher in the $\mathrm{GFP}^{+}$cells isolated from EHM as compared to the input $\mathrm{GFP}^{+}$populations. A separate qPCR amplification of RFP (unique cardiomyocyte label) suggested carryover during the separation of the $\mathrm{GFP}^{+}$cell pools by FACS (Figure 32C). This notion was further supported by fluorescence microscopy of the separated cell pools with little, but detectable contaminating $\mathrm{RFP}^{+}$cells in the $\mathrm{GFP}^{+}$-sorted cell population (Figure 32D). Taken together and despite some hints for non-myocyte differentiation or fusion we conclude that the most likely explanation for $\mathrm{RFP}^{+} /$actinin $^{+}$cells in the $\mathrm{GFP}^{+}$hFF and hCPC pools would be cardiomyocyte carry-over. Single cell PCR and RNA-sequencing technologies may help to further identify whether transdifferentiation of hFFs or hCPCs had occurred in EHM. Nonetheless, it appears unlikely that these rare events contributed to the cardio-supportive effects of the nonmyocyte pools in EHM. 

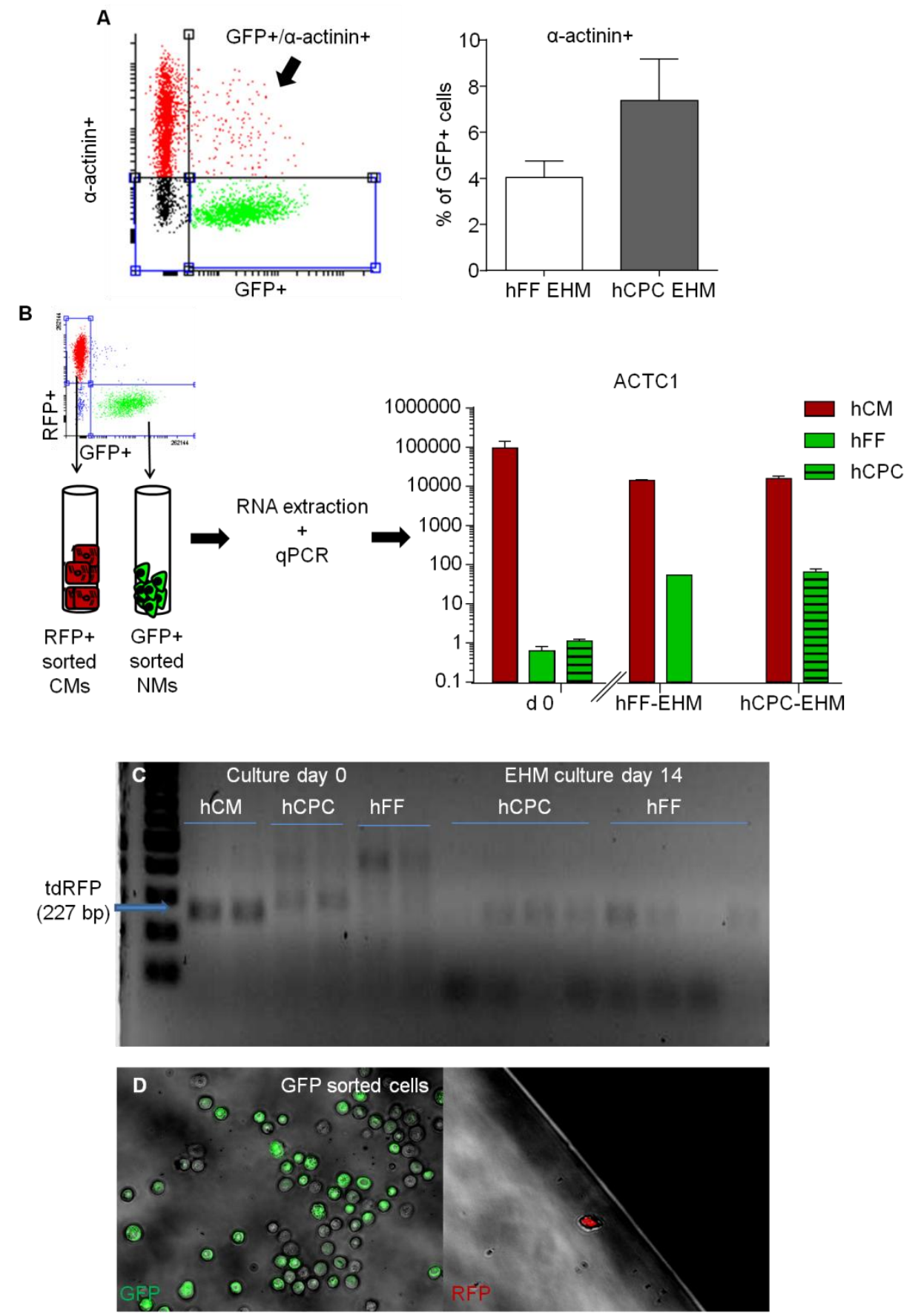

Figure 32. Strategies to investigate cardiac differentiation of hCPCs in EHM. A Flow cytometry analysis of $\mathrm{GFP}^{+} / \alpha$-actinin ${ }^{+}$cells digested from either hFF- or hCPC-EHM ( $n=6 /$ group from four experiments). B FACS of $\mathrm{RFP}^{+} \mathrm{CMs}$ (cardiomyocytes) and $\mathrm{GFP}^{+} \mathrm{NMs}$ (non-myocytes) after digestion from EHM co-cultures and GPCR analysis of cardiac actin gene expression in the separately sorted pools (normalized to $h F F s$ at culture day $0 ; n=2 /$ group). C Electrophoretic separation of RFP transcripts (specific band at $227 \mathrm{bp}$ ) after qPCR amplification in hCM, hCPC and hFFs at culture day 0 and in GFP-sorted hCPCs and hFFs at EHM culture day 14. D GFP and RFP fluorescence signals on GFP-sorted cells after flow cytometry sorting. 


\subsection{Modeling hypoxic injury in EHM}

Experimental hypoxia/reoxygenation $(H / R)$ models are useful tools to investigate cardio-protective strategies against myocardial infarction (Portal et al. 2013). To investigate cardio-protective effect of hCPCs, the aim was to first develop EHM models of acute and chronic hypoxia-dependent injury.

\subsubsection{Hypoxia response in human cardiomyocytes}

To characterize the effect of hypoxia on the physiological state of cardiomyocytes, HIF-1 $\alpha$ protein stability and metabolic modifications were analyzed under hypoxia $\left(1 \% \mathrm{O}_{2}\right)$ exposure for 8-72 hrs. Cardiomyocytes responded to hypoxia with HIF-1a stabilization after $8 \mathrm{hrs}$ of hypoxia exposure (Figure 33A). Metabolic activity changed accordingly as exemplified by a drop in intracellular ATP amount (Figure 33B). In agreement with these findings, a gradual increase in lactate was detected starting after 8 hrs of hypoxia (Figure 33C). Accumulation of lactate suggests that human cardiomyocytes shift their energy metabolism from aerobic respiration to anaerobic gycolysis. Constant ATP levels at 8 and $24 \mathrm{hrs}$ of hypoxia suggested that the cardiomyocytes seem to adapt their metabolism to hypoxia within this time frame. Extended hypoxia (72 hrs) appeared to cause hypoxic damage, leading to a drop in ATP synthesis with strong lactate accumulation (Figure 33B,C). 

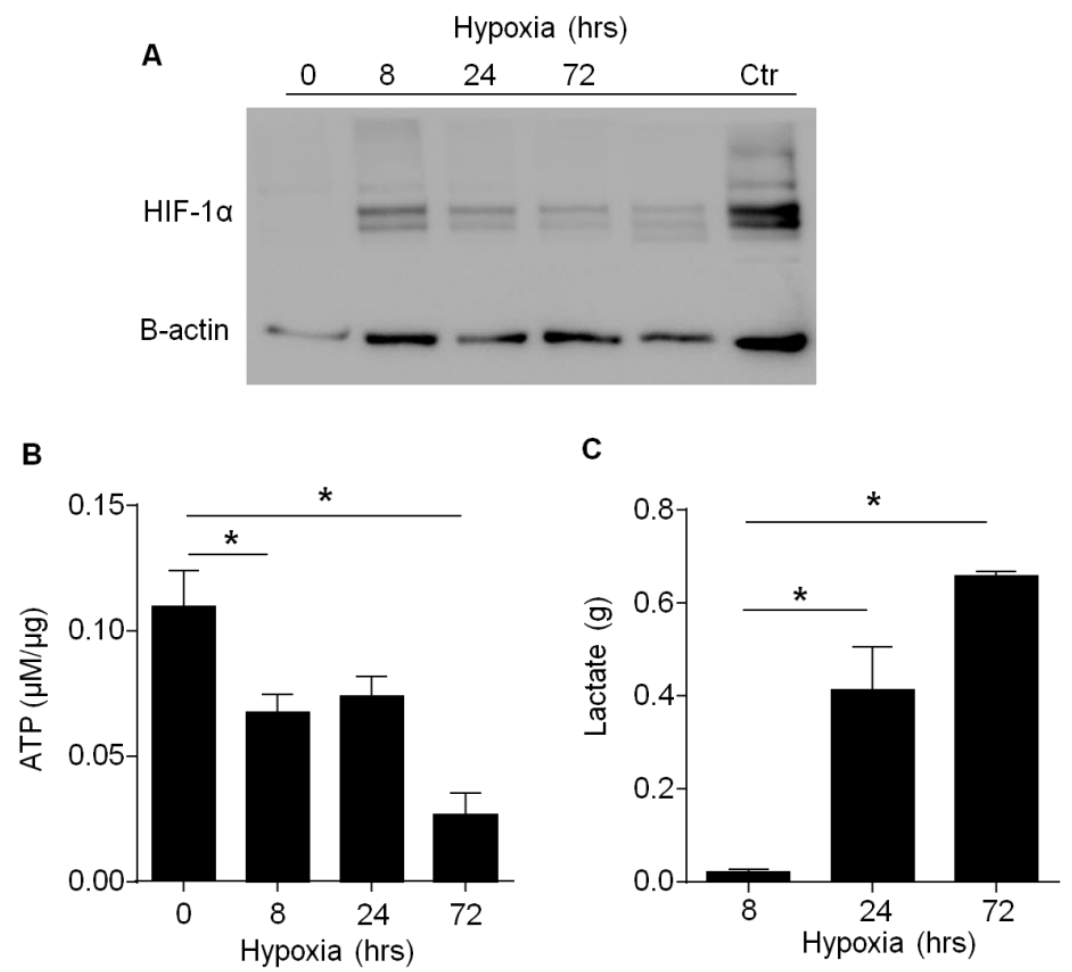

Figure 33. Metabolic adaptation of human cardiomyocytes under hypoxia. A HIF-1a protein abundance by Western blot detection. B Intracellular ATP amount and $\mathbf{C}$ released lactate into the culture medium ( $n=4-5 /$ time-point; ${ }^{*}<<0.05$ as indicated by one-way ANOVA, Dunnett's multiple comparison test).

\subsubsection{Hypoxia response in human EHM}

hFF-EHMs were exposed to $1 \% \mathrm{O}_{2}$ for 8-120 hrs. Lactate release was increased after $8 \mathrm{hrs}$ of hypoxia, reaching maximal levels at $72 \mathrm{hrs}$ (Figure 34). This was in line with the lactate release profile observed in human cardiomyocytes under hypoxia. Note that lactate release was also observed in EHM developed under $21 \% \mathrm{O}_{2}$ ("normoxia") without medium exchange reaching similar levels as observed in the hypoxic EHM after $120 \mathrm{hrs}$ in culture. Thus, the hypoxia related cell damage occured within the first $96 \mathrm{hrs}$ of $\mathrm{hEHM}$ culture at $1 \% \mathrm{O}_{2}$. Beyond that time window, nutrient depletion may have contributed to general cell damage. 


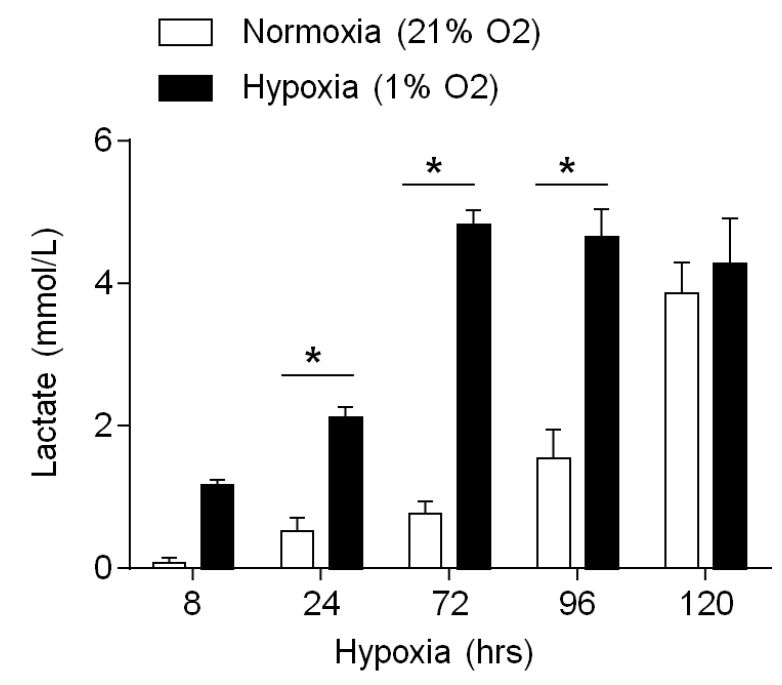

Figure 34. Lactate production by hEHM cultured under normoxia and hypoxia. Lactate accumulation in hEHM culture medium under normoxia $\left(21 \% \mathrm{O}_{2}\right)$ and hypoxia $\left(1 \% \mathrm{O}_{2}\right)$ for $8-120 \mathrm{hrs}$ with no medium change ( $n=4 /$ group, ${ }^{*} p<0.05$ by two-way ANOVA with Sidak's multiple comparison test).

\subsubsection{Hypoxia/Reoxygenation damage in human EHM}

hEHMs were exposed to different durations of hypoxia $\left(\mathrm{H} ; 1 \% \mathrm{O}_{2} ; 8-120\right.$ hours $)$ followed by $24 \mathrm{hrs}$ of reoxygenation $\left(R ; 21 \% \mathrm{O}_{2}\right)$ to identify whether hypoxia/reoxygenation $(H / R)$ damage could be simulated (Figure 35A). We did not observe any reduction in FOC of hEHMs under hypoxia for 8-72 hrs (8 and 24 hrs time points not shown). However, reoxygenation after 72 hrs hypoxia resulted in a marked reduction in EHM contractility (Figure 35B). Conversely, $120 \mathrm{hrs}$ of hypoxia caused EHM damage, which could not be further aggravated by reoxygenation (Figure 35C). 
A

Hypoxia $\left(1 \% \mathrm{O}_{2}\right)$

$+24 \mathrm{hrs}$

Reoxygenation

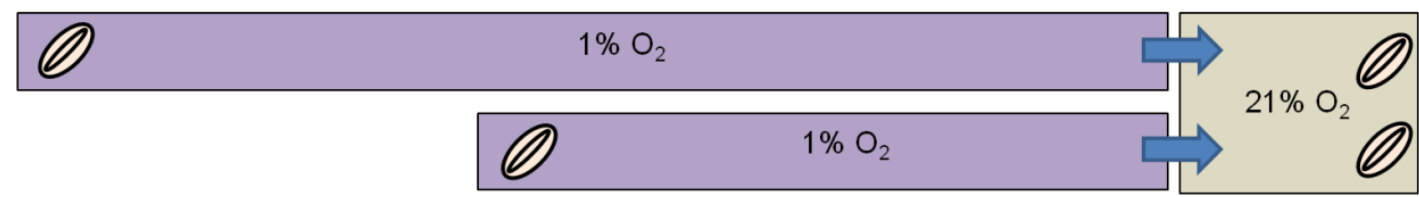

+ Medium change

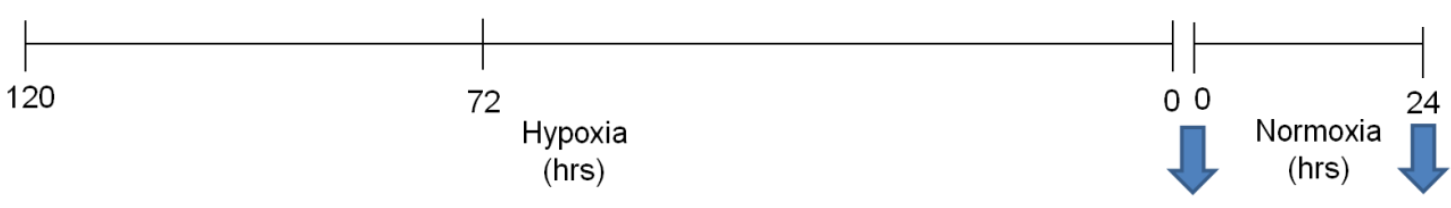

-Force of contraction

-CM amount

-CM structure and function

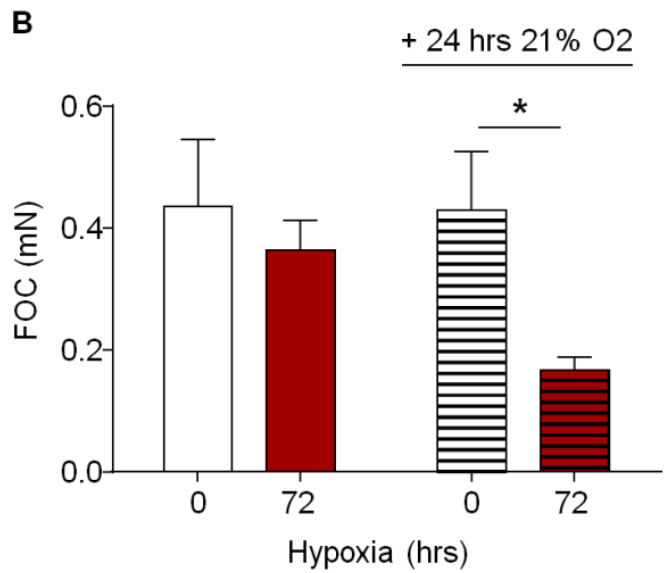

C

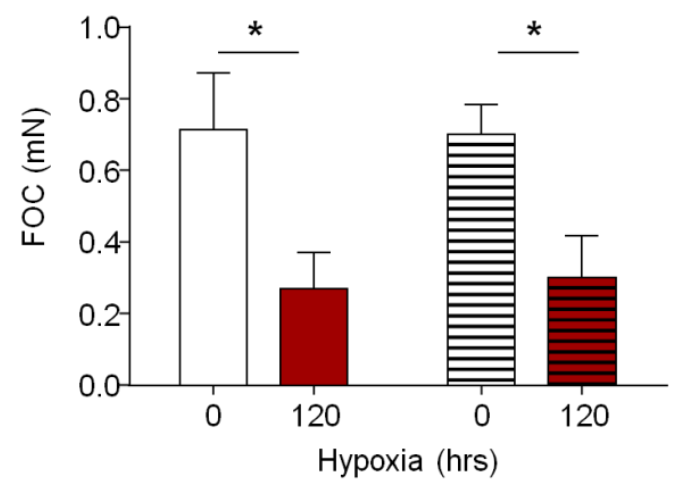

Figure 35. Development of a hypoxia/reoxygenation injury hEHM model. A Schematic overview of the experimental setting of $\mathrm{H} / \mathrm{R}$ injury in hEHMs. hEHMs incubated in $1 \% \mathrm{O}_{2}$ for 72 or $120 \mathrm{hrs}$ and reoxygenated in $21 \% \mathrm{O}_{2}$ in an oxygenated fresh medium for $24 \mathrm{hrs}$ and subsequently analyzed at functional and structural level. B Force of contraction (FOC) in hEHM after only 72 hrs of hypoxia or 72 hrs hypoxia followed by $24 \mathrm{hrs}$ of reoxygenation ( $\mathrm{n}=5-6 /$ group from two experiments, unpaired $t$-test, $\left.{ }^{*} \mathrm{p}<0.05\right)$. C FOC in hEHM after only $120 \mathrm{hrs}$ of hypoxia or $120 \mathrm{hrs}$ of hypoxia, followed by $24 \mathrm{hrs}$ of reoxygenation ( $n=8 /$ group from two experiments, unpaired $t$-test, ${ }^{*} \mathrm{p}<0.05$ ). hEHMs at $0 \mathrm{hr}$ of hypoxia served as normoxia control for the corresponding experimental conditions.

Interestingly, cardiomyocyte quantity per EHM was significantly reduced after 72 hrs (Figure 36A), without an obvious impact on EHM contractility (Figure 35B). Reoxygenation aggravated the cardiomyocyte loss after $72 \mathrm{hrs}$ of hypoxia (Figure 36A), which was in-line with the observed functional decay (Figure 35B). As expected, chronic hypoxia stimulation (120 hrs) resulted in a pronounced decrease in cardiomyocyte numbers, (Figure 36A) with at best a tendency to enhanced 
cardiomyocyte survival after enzymatic digestion from EHM subjected to $24 \mathrm{hrs}$ of reoxygenation. Note that the discrepancy between contractile performance and cardiomyocytes count after 72 hrs hypoxia could result from a higher susceptibility of hypoxic cardiomyocytes to enzymatic EHM digestion. We further employed a LDH activity assay to evaluate cell damage upon reoxygenation and found that extended reoxygenation (24 hrs) caused substantial damage (Figure 36B).
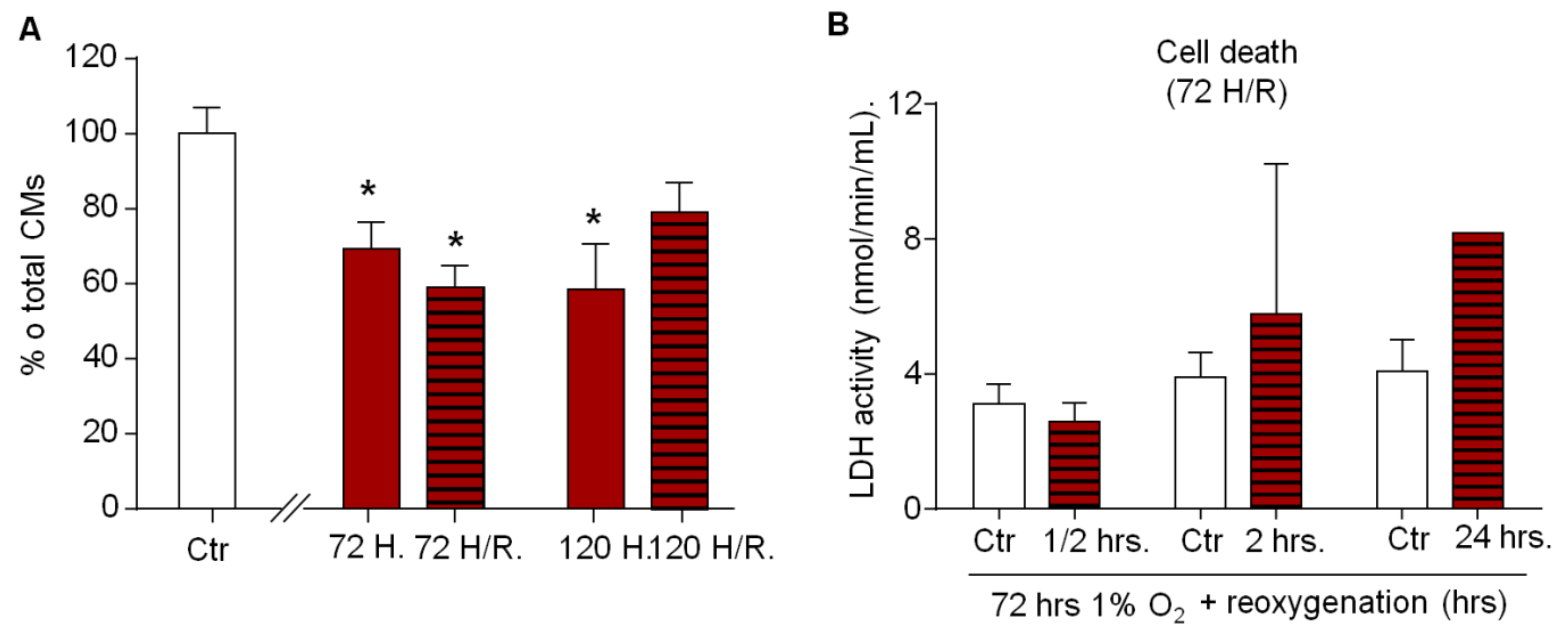

Figure 36. Validation of the hypoxia/reoxygenation injury hEHM model. A Percentage of total cardiomyocytes after $72 \mathrm{hrs}\left(72 \mathrm{H} ; \mathrm{n}=3-4 /\right.$ group, unpaired $t$-test, $\left.{ }^{*} \mathrm{p}<0.05\right)$ and after $120 \mathrm{hrs}$ of hypoxia $\left(120 \mathrm{H} ; \mathrm{n}=6-8 /\right.$ group, unpaired $t$-test, ${ }^{*} \mathrm{p}<0.05$ vs. Ctr), with the following $24 \mathrm{hrs}$ of reoxygenation induction $(72 \mathrm{H} / \mathrm{R}$ or $120 \mathrm{H} / \mathrm{R})$. B LDH activity as an indicator of cell damage/death during $24 \mathrm{hrs}$ of reoxygenation following 72 hrs of hypoxia ( $n=2-3 / g r o u p)$. Ctr: Control EHM incubated under normoxia for the corresponding experimental condition.

Collectively, these data define the time windows for the simulation of hypoxic (120 hrs $1 \%$ O2) and hypoxia/reoxygenation (72 hrs 1\% O2 followed $24 \mathrm{hrs} 21 \%$ O2) damage in human EHM.

\subsection{Assessment of the protective effects of human CPCs in EHM}

hCPCs are tested clinically for myocardial protection after myocardial infarction (Makkar et al. 2012). Here, we aimed at investigating potential cardio-protective effects of hCPCs in the EHM hypoxia/reoxygenation and chronic hypoxia injury models. 


\subsubsection{No evidence for paracrine protection by CPCs}

hEHMs were exposed to $H / R$ injury as established before (see 3.5.3) and exposed to either hCPC- or hFF-conditioned medium (ConM) during reoxygenation after 4 weeks of regular EHM culture (Figure 37A). As expected, H/R significantly reduced hEHM function markedly compared to normoxia control EHM (EHM-Ctr: continuously incubated at $21 \% \mathrm{O}_{2}$ ). hEHM treated with hCPC-ConM did not show any rescue from $\mathrm{H} / \mathrm{R}$ injury. Interestingly, hFF-ConM treatment during reoxygenation partially preserved contractile function of $\mathrm{hEHM}$, reducing the injury effect by $\sim 50 \%$ (Figure 37B). $H / R$ injured hEHMs (EHM H/R: treated with fresh medium) showed increased calcium sensitivity ( $E_{50}$ : Ctr. $0.7 \pm 0.1, n=9 /$ group vs. $H / R 0.3 \pm 0.1 \mathrm{mmol} / \mathrm{L}, \mathrm{n}=4 /$ group, $p<0.05$ by Student's $t$-test; Figure $37 \mathrm{C}$ ). hFF-ConM and hCPC-ConM shifted the calcium sensitivity in $\mathrm{H} / \mathrm{R}$ towards Ctr levels with a slightly more enhanced effect in hFF-ConM (fold difference in $\mathrm{EC}_{50}$ in mmol/L: hFF-ConM 0.6 $\pm 0.1, \mathrm{n}=9$ /group vs. hCPC-ConM $0.5 \pm 0.1 \mathrm{mmol} / \mathrm{L}, \mathrm{n}=5 /$ group; Figure $37 \mathrm{C}$ ). The latter finding needs further investigations on the molecular level to determine whether calciumhomeostasis is indeed altered and involved in the functional deterioration as well as protection in $\mathrm{H} / \mathrm{R} \mathrm{EHM}$. 
A
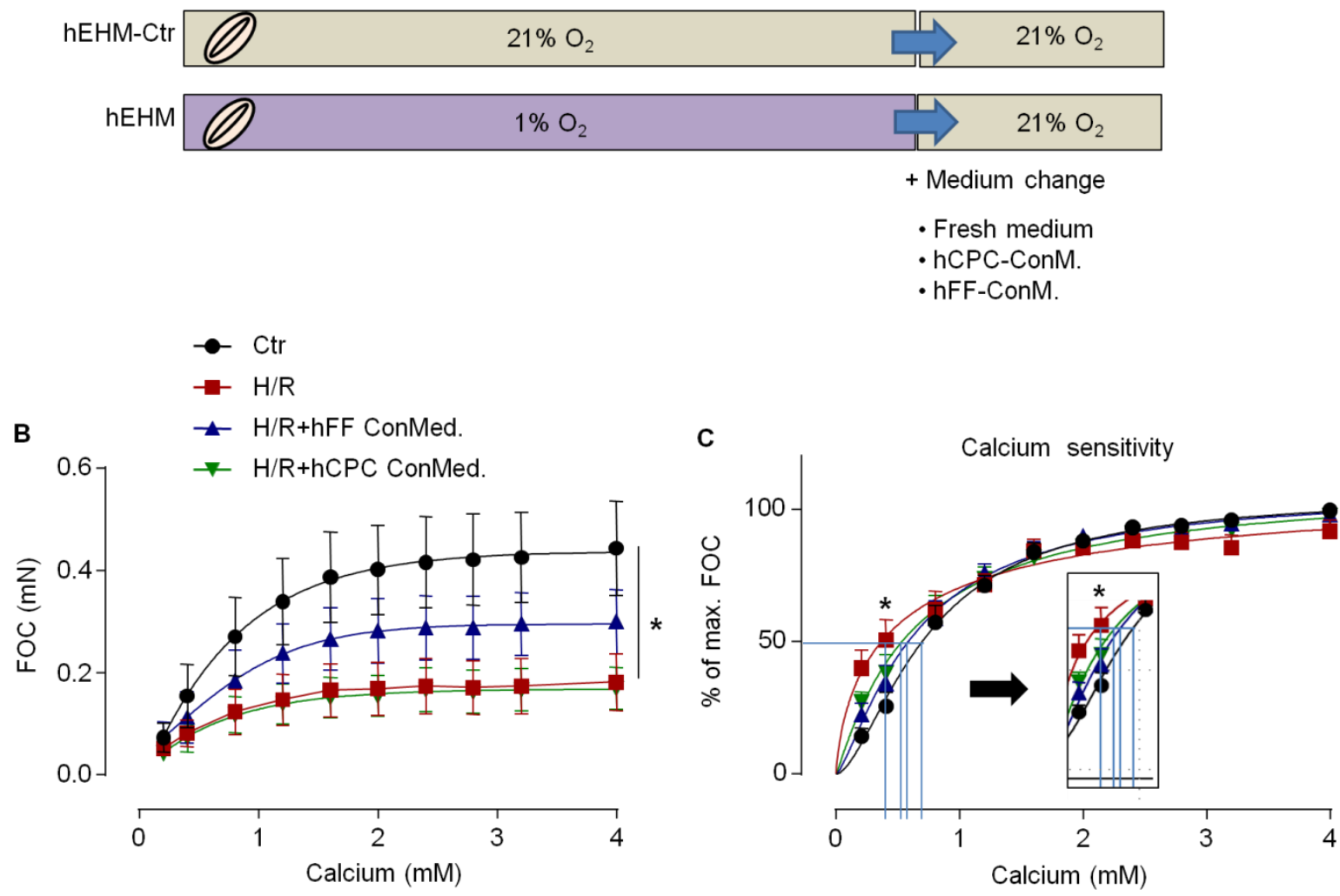

Figure 37. Effects of conditioned medium on EHM function upon $H / R$ injury. A Schematic overview of the experimental design to test potential protective mechanisms of hCPC-conditioned culture medium. Following $72 \mathrm{hrs}$ of hypoxia, hEHMs were exposed to $24 \mathrm{hrs}$ of reoxygenation in the presence of hCPC- or hFF-Conditioned medium (ConM); regular medium exchange in the H/R model served as "injury" control; standard hEHM cultures served as "healthy" controls. B Force of contraction (FOC) and C calcium sensitivity in Ctr (normoxia), H/R (H/R injury), H/R+hCPC-ConM and hEHM$\mathrm{H} / \mathrm{R}+\mathrm{hFF}-\mathrm{ConM}$ ( $n=9-10 / \mathrm{EHM}$ from three experiments, ${ }^{*} \mathrm{p}<0.05$ vs. Ctr. by two-way ANOVA, Dunnett's multiple comparison test $\mathbf{B}$ and Student's $t$-test $\mathbf{C}$.

Consistent with the data on EHM function (Figure 37B), H/R caused a significant reduction in cardiomyocte content and reduced contractile function. Similarly as reported for EHM function, there was no protection from cardiomyocyte death upon H/R injury by hCPC-ConM. Conversely, cardiomyocyte amount and contractility as a fuction of cardiomyocyte number was markedly enhanced in hFF-ConM treated hEHMs (Figure 38A,B), suggesting that hFFs rather than hCPCs were able to protect cardiomyocytes from $\mathrm{H} / \mathrm{R}$ by paracrine mechanisms. 
A

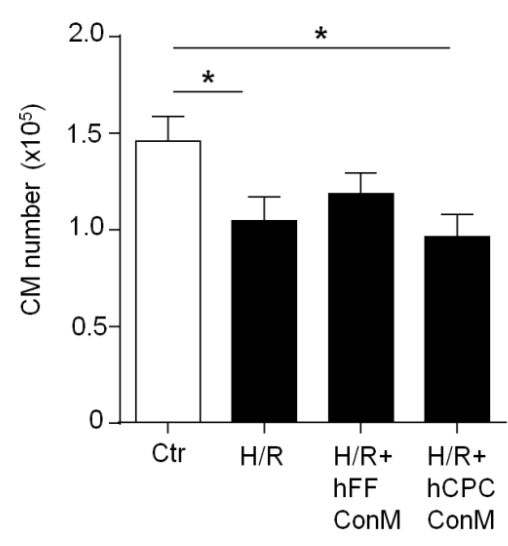

B

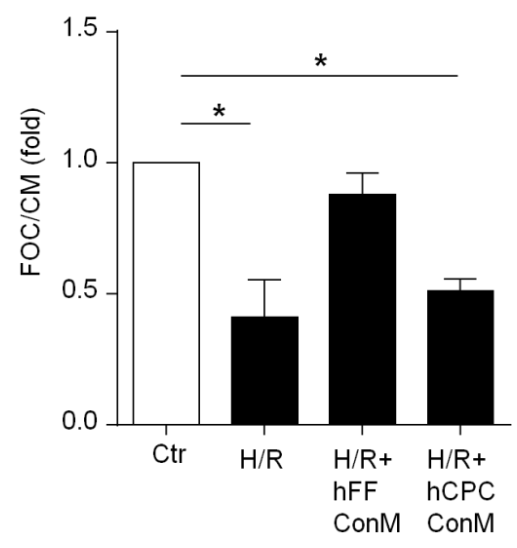

Figure 38. Effects of conditioned medium on EHM cardiomyocyte content upon $H / R$ injury. A Cardiomyocyte number ( $n=8-10 /$ group) and B force of contraction (FOC) as a function of cardiomyocyte content in Ctr, H/R, H/R+hFF-ConM and H/R+hCPC-ConM EHM. $n=3 /$ group; ${ }^{*} p<0.05$ vs. Ctr by one-way ANOVA with Dunnett's post-hoc test.

To investigate whether $H / R$ impaired the responsiveness to $\beta$-adrenergic stimulation the response to maximally effective isoprenaline $(1 \mu \mathrm{mol} / \mathrm{L})$ was tested. These analyses showed a markedly depressed isoprenaline response in $H / R$ treated $E H M$ irrespective of the attempted conditioned medium rescue (Figure 39).

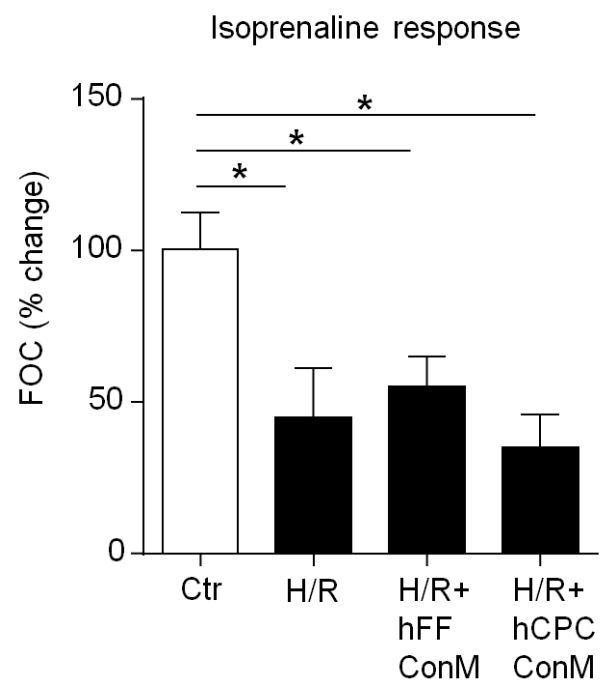

Figure 39. Impaired $\beta$-adrenergic signaling in hEHM after H/R injury. Force of contraction (FOC) in percent of the inotropic effect induced by isoprenaline in control $E H M$ at $E_{50}$ calcium concentrations; $n=9-10 /$ group; ${ }^{*}<<0.05$ vs. Ctr by one-way ANOVA with Dunnett's post-hoc test. 


\subsubsection{Discrimination of paracrine effects by transcriptome profiling}

CPCs have been reported to possess paracrine potency to reduce cell death and support cardiomyocyte proliferation as well as play angiogenic (Chimenti et al. 2010, Li et al. 2012, Malliaras et al. 2013) and anti-inflammatory roles (Liu et al. 2014). To gain preliminary insight into the specific paracrine activity of hCPCs and hFFs and to explain the differential effect of conditioned medium, a number of growth factors and cytokines that are involved in cell survival, proliferation, angiogenesis and inflammation (see Appendix A6 for the full list of growth factors and cytokines) were selected and screened in the pool of differentially expressed genes from RNAseq data in hCPCs vs hFFs. Several clusters of genes were detected that were differentially expressed between hCPCs and hFFs (Figure 40A). Ontology enrichment identified that hCPC genes involved in angiogenesis and immune response were strongly regulated. Paracrine factors up-regulated in hFFs were more involved in the regulation of cell proliferation and survival (Figure 40A). We also confirmed the expression of the specific growth factors FGF-2, VEGF, IGF-1, HGF and PDGF - all with a known effect on cell proliferation, survival and migration - by PCR (Figure 40B). The enrichment of transcripts encoding for cell survival enhancing factors in hFFs was in line with their effects on cardiomyocyte survival in EHM upon $\mathrm{H} / \mathrm{R}$ injury (Figure 38A). Given the major effect of hFF-ConM on the protection of cardiomyocyte function after $H / R$ injury, it might be worth to investigate the mechanism behind the impairment in cardiac function, first and further screen for related cardio-supporting factors secreted from these fibroblasts. On the other hand, possible pro-angiogenic and inflammation modulatory role of hCPCs according to the RNAseq data might provide a possible mechanism for their ameliorating effect on tissue injury in vivo (Liu et al. 2014). No effect in EHM may be attributed to the lack of vascular cells and leukocytes in EHM. 
A
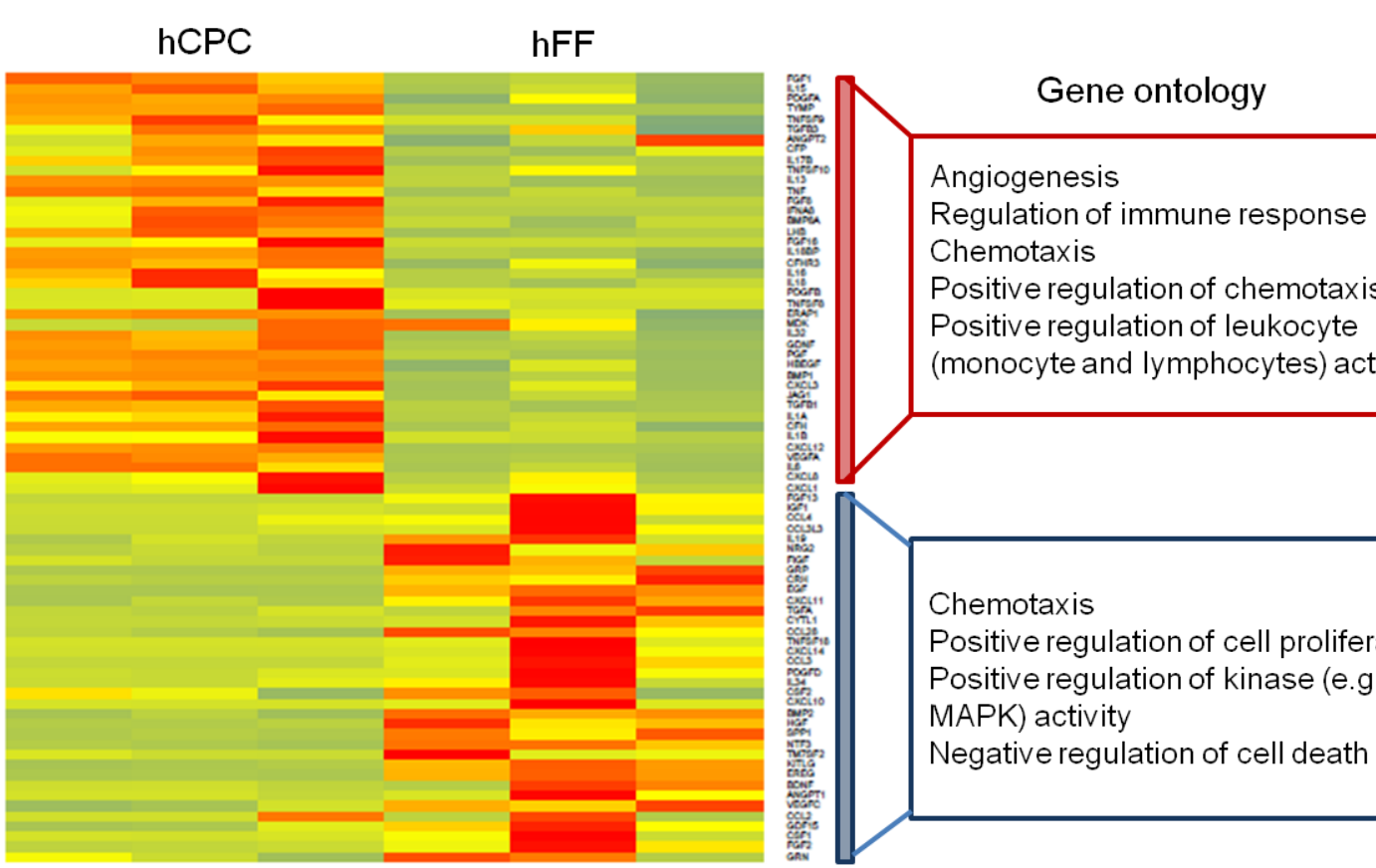

Regulation of immune response Chemotaxis

Positive regulation of chemotaxis

Positive regulation of leukocyte

(monocyte and lymphocytes) activation

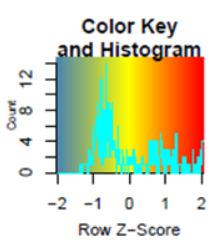

Chemotaxis

Positive regulation of cell proliferation

Positive regulation of kinase (e.g.

MAPK) activity

Negative regulation of cell death

B

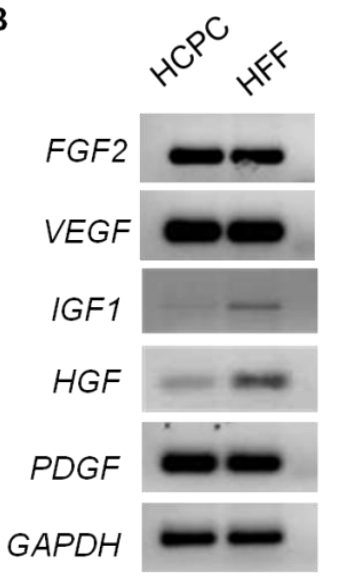

Figure 40. Paracrine activity in hCPCs and hFFs. Heatmap analysis of genes encoding for paracrine factors involved in cell survival, proliferation, angiogenesis and inflammation in hCPCs and hFFs; on the right functional annotation of differentially expressed genes in gene clusters; Red and blue reflect high and low expression levels, respectively as scaled in color key bar ( $n=3 /$ group). B PCR analysis of a selected number of transcripts encoding for growth factors with implications in cardiac homeostasis: FGF2, VEGF, IGF1, HGF and PDGF; GAPDH served as housekeeping reference gene. 


\subsubsection{Cardio-protective effects of CPCs in EHM exposed to chronic hypoxia}

To investigate whether or not hCPCs elicit a cardio-protective role under chronic hypoxia and following the hypothesis that hCPCs may elicit their effects only under more physiological conditions, EHMs were constructed from CMs (65\%) and hFFs (28\%) with the addition of hCPCs (7\%) and exposed to $1 \%$ oxygen for $120 \mathrm{hrs}$ after 4 weeks of regular EHM culture (Figure 41A). Chronic hypoxia decreased FOC by almost $68 \pm 6 \%$ in hFF-EHM in agreement with the findings reported before (refer to Figure 35C). Interestingly, hFF+hCPC-EHM already under normoxic control conditions presented with slightly enhanced forces and an only mildly reduced FOC under chronic hypoxia (Figure 41B). Calcium sensitivity (average $\mathrm{EC}_{50}: 0.34 \pm 0.03$ $\mathrm{mmol} / \mathrm{L}$ ) was similar in all groups suggesting differential effects of $H / R$ and chronic hypoxia on calcium homeostasis (Figure 41C). 
A 120 hrs Hyp.

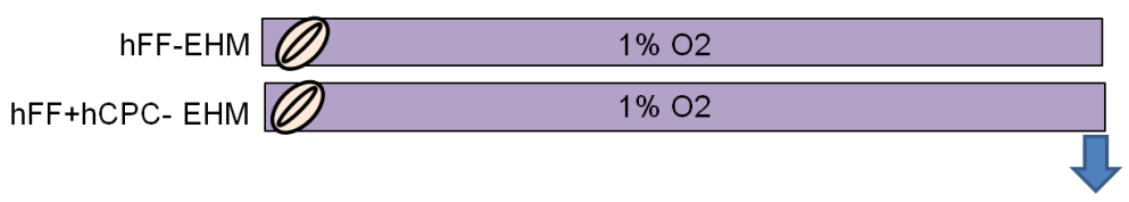

-Force of contraction

-CM amount

-CM structure and function

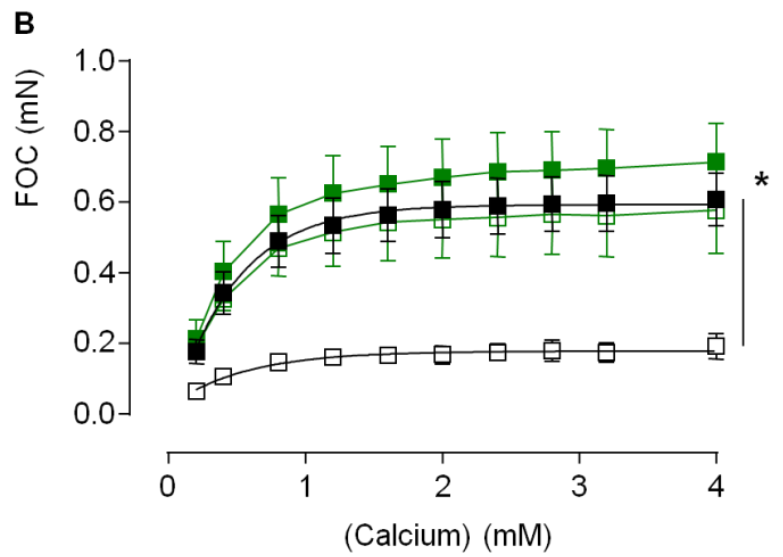

C

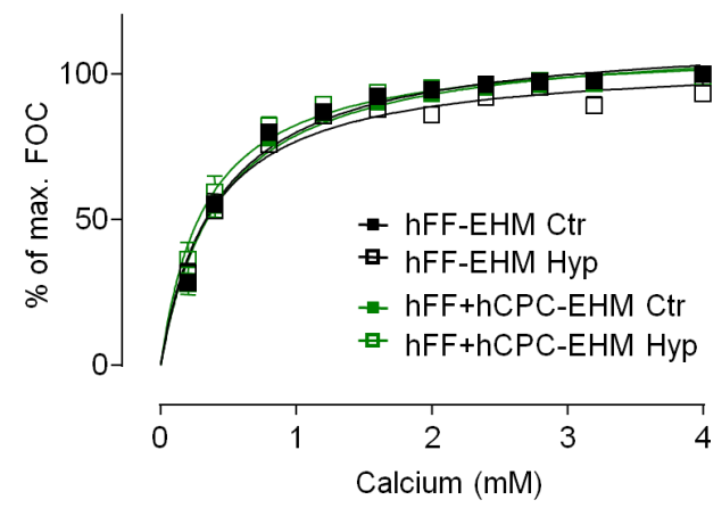

Figure 41. Effects of CPC in EHM tri-cultures upon chronic hypoxia. A Schematic overview of the experimental design to test potential protective mechanisms of hCPCs on EHM composed of hFFs and CMs with or without hCPC supplementation. Following 4 weeks of regular culture, EHMs were exposed to $1 \% \mathrm{O}_{2}$ for 120 hrs (hypoxia: Hyp) or maintained under $21 \%$ ambient $\mathrm{O}_{2}$ (Ctr) without culture medium exchange; hFF-EHM: 70\% CMs and 30\% hFFs; hFF+CPC-EHM: 65\% CMs, 28\% hFFs, 7\%CPCs. B Force of contraction (FOC) and $\mathbf{C}$ calcium sensitivity in $\mathrm{Ctr}$ in the indicated groups; $n=4 /$ group, ${ }^{*} p<0.05$ by two-way ANOVA with Tukey's multiple comparison post hoc test.

No obvious difference was detected in cardiomyocyte amount between hFF- and hFF+hCPC-EHM under normal conditions. After chronic hypoxia, cardiomyocyte content was significantly decreased in hEHM with or without hCPC supplementation (Figure 42A); however, a trend to a better cardiomyocyte preservation was apparent in the hCPC supplemented EHMs. a-Actinin content and function per cardiomyocyte were preserved only in hFF+hCPC-EHM, suggesting a beneficial effect of hCPCs on cardiomyocytes in EHM tri-cultures (Figure 42B,C). Further investigations are needed to identify the apparently cell context dependent effects of CPCs. 

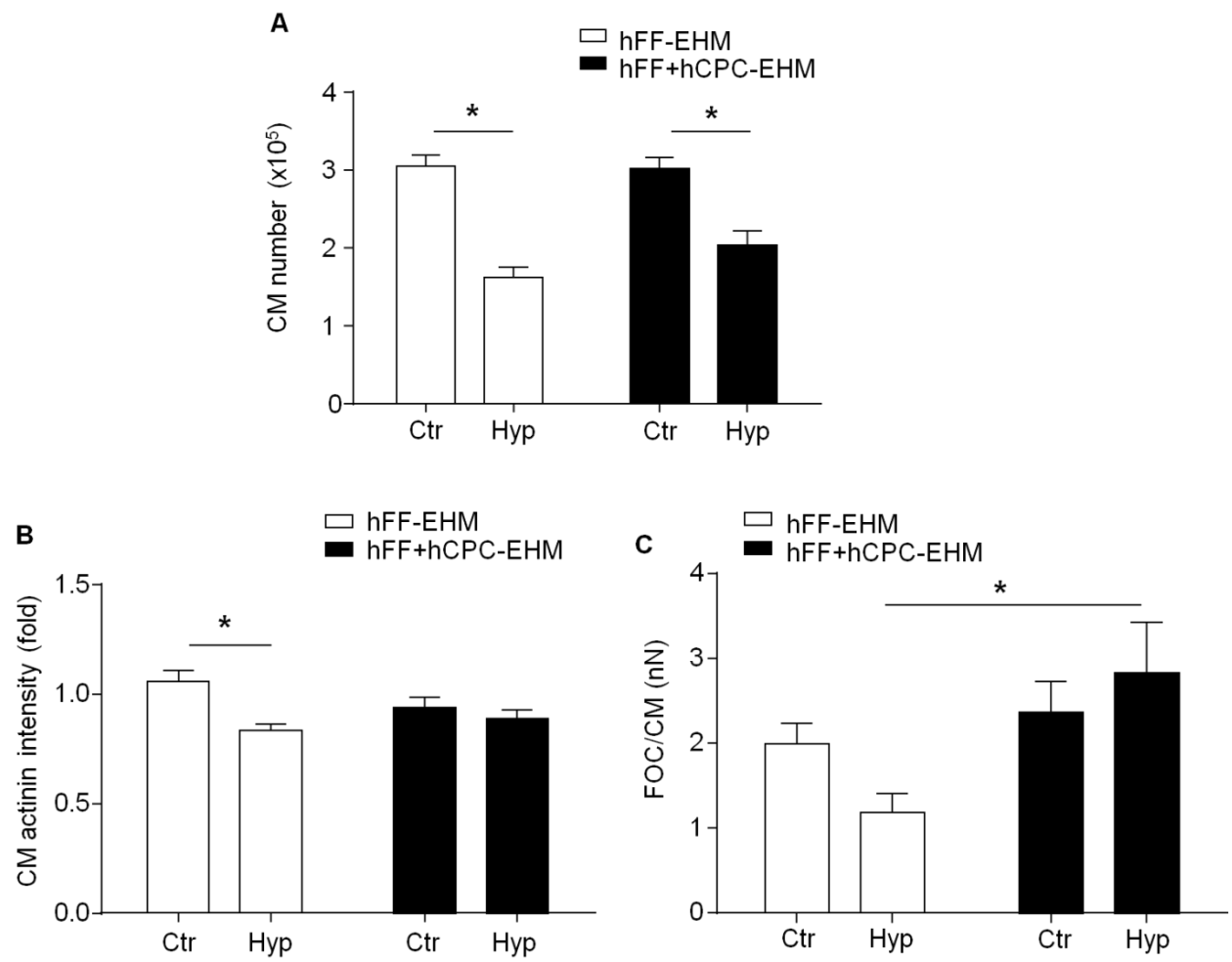

Figure 42. Enhanced cardiomyocyte survival and function in EHM tri-cultures. Experimental conditions were as outlined in Figure 41A with EHM exposed to chronic hypoxia. A Cardiomyocyte number/EHM, B cardiomyocyte $\alpha$-actinin content by mean fluorescence intensity measurements and C FOC as a function of EHM cardiomyocyte content ( $n=4 /$ group same EHM as in Figure 46 ); ${ }^{*} p<0.05$ as indicated by two-tailed, unpaired Student's $t$-test.

\subsection{Development of a genetic hypoxia indicator model}

Cell and tissue hypoxia models would benefit greatly from tools for life-cell imaging of cell oxygenation states. First steps towards the ultimate goal to develop a hEHM model comprising cardiomyocyte-specific hypoxia sensors are described below.

\subsubsection{Hypoxia sensing in ODD-Luc mouse cardiomyocytes}

In order to identify the biological response of cardiomyocytes to hypoxia, a genetically modified mouse ODD-Luc ESC line (see Appendix A4) was utilized to generate ODDLuc mouse cardiomyocytes. The ubiquitously expressed reporter in this ODD-Luc ESC line comprised a HIF-1 $\alpha$ oxygen-dependent degradation domain (ODD) fused to a firefly luciferase (Luc). Stability of the ODD-Luc fusion protein depends on the oxygenation level. Under normoxia, prolyl residues in the ODD domain are hydroxylated by prolyl-4-hydroxylase-domain (PHD) enzymes, which subsequently 
results in ubiquitination and rapid degradation of the protein. Under hypoxia, reduced PHD enzyme activity leads to stabilization of HIF-1a (Schofield and Ratcliffe 2004). According to this system, any decrease in oxygen amount should lead to stabilization of the ODD-Luc protein and thus an increase in Luc signal intensity (Figure 43A).

To validate whether the response to hypoxia can indeed be measured in this cellbased reporter model, ODD-Luc cardiomyocytes were exposed to $1 \% \mathrm{O}_{2}$ for $1-72 \mathrm{hrs}$ and Luc signal was analyzed at multiple time-points. ODD-Luc cardiomyocytes showed a significant increase in Luc signal with peak intensity at 24 hrs (Figure 43B). Luc signal intensity showed a good correlation with the abundance of ODD-LuC protein and endogenous HIF-1a protein (Figure 43C). Elevation of PHD2 protein at $24 \mathrm{hrs}$, as a negative feedback mechanism may be an additional explanation for the decrease in endogenous HIF-1 $\alpha$ and ODD-Luc protein in extended culture (Figure 43C). Collectively, these findings support the use of the ODD-Luc model in sensing hypoxia in cardiomyocytes. 

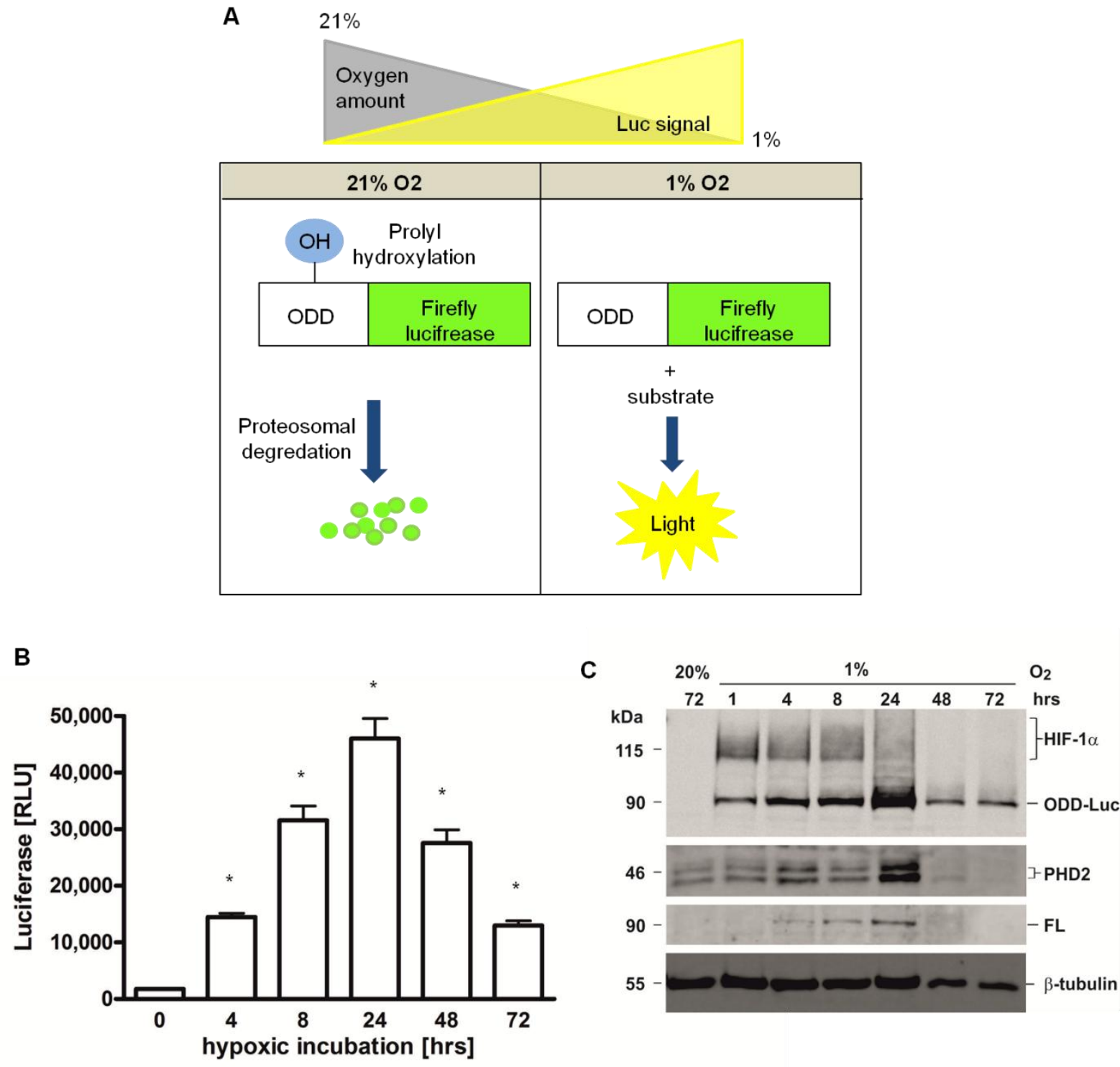

Figure 43. Validation of ODD-Luc hypoxia reporter in cardiomyocyte culture. A Schematic representation of the mechanisms regulating the stability of ODD-Luc fusion protein based on ambient oxygen levels $\left(21 \% \mathrm{O}_{2}\right.$ : hydroxylation of prolyl residues in HIF-1 $\alpha-O D D$ results in proteosomal degradation of the ODD-Luc fusion protein, $1 \% \mathrm{O}_{2}$ : no post-translational modification in HIF-1a-ODD and stabilization of ODD-Luc fusion protein). B Time-dependent Luc signal development in antibiotically selected ODD-Luc cardiomyocytes (mouse model) upon exposure to $1 \% \mathrm{O}_{2}$ for $1-72 \mathrm{hrs}$ ( $n=6 /$ group). C Protein expression of endogenous HIF-1a, ODD-Luc fusion protein, PHD2 protein, firefly luciferase $(\mathrm{FL})$ and $\beta$-tubulin using western blot (provided by Anke Zeiseniss; Institute of Cardiovascular Physiology, UMG). Data was published in (Hesse et al. 2014). 


\subsubsection{Hypoxia sensing in ODD-Luc mouse EHM}

ODD-Luc cardiomyocytes were utilized to generate EHMs with genetically naïve fibroblasts, based on the mouse EHM protocol as described previously (section 2.3.2). ODD-Luc EHMs showed the highest signal at 24-48 hrs after the induction of hypoxia with a subsequent signal decrease (Figure 44A). Reoxygenation of ODDLuc EHMs at $21 \% \mathrm{O}_{2}$ after $4 \mathrm{hrs}$ of hypoxia rapidly diminished the Luc signal intensity with an apparent half-life of $15 \pm 3 \min (n=3)$ for the ODD-Luc protein complex (Figure 44B)
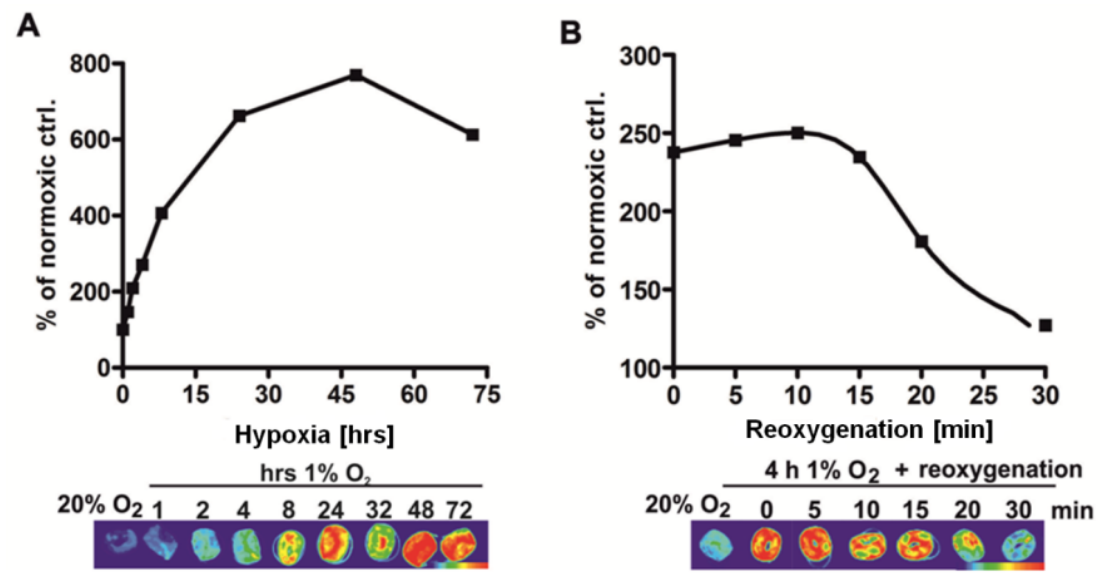

Figure 44. Hypoxia and repoxygenation responses in ODD-Luc EHM. A Time-dependent Luc signal development in ODD-Luc EHM during hypoxia $\left(1 \% \mathrm{O}_{2}\right)$ for 1-72 $\mathrm{hrs}(\mathrm{n}=3 /$ time point). B Timedependent reduction of Luc signal in ODD-Luc EHM after reoxygenation following $4 \mathrm{hrs}$ of hypoxia ( $n=3 /$ time-point). Panels below display signals recorded from representative ODD-Luc EHM (blue indicates low and red indicates high Luc signal). Data was published in (Hesse et al. 2014).

\subsubsection{Hypoxia sensing in human cardiomyocytes}

After obtaining proof-of-concept in the mouse model that the ODD-Luc reporter system can be used as a tool to monitor tissue oxygenation and hypoxia response, we attempted to establish the same system in a human model. For this purpose, ODD-Luc hESCs were generated using TALEN technology. Briefly, wild-type hESCs were targeted with pAAVS1-CAG-ODD-Luc-EF1a-GFP donor vector (section 2.8), which was generated by Dr. Claudia Noack and pAAVS1 TALEN Left and Right vectors. Resulting ODD-Luc expressing hES cells were differentiated into ODD-Luc 
expressing human cardiomyocytes (ODD-Luc hCMs). ODD-Luc hCMs were validated for their hypoxic response with significantly higher expression of Luc signal after $4 \mathrm{hrs}$ of hypoxia $\left(1 \% \mathrm{O}_{2}\right)$ exposure (Figure 45). In future experiments, we intend to use these ODD-Luc hCMs to generate ODD-Luc hEHMs as a human heart muscle model to gain a better understanding on their response to hypoxia and reoxygenation.

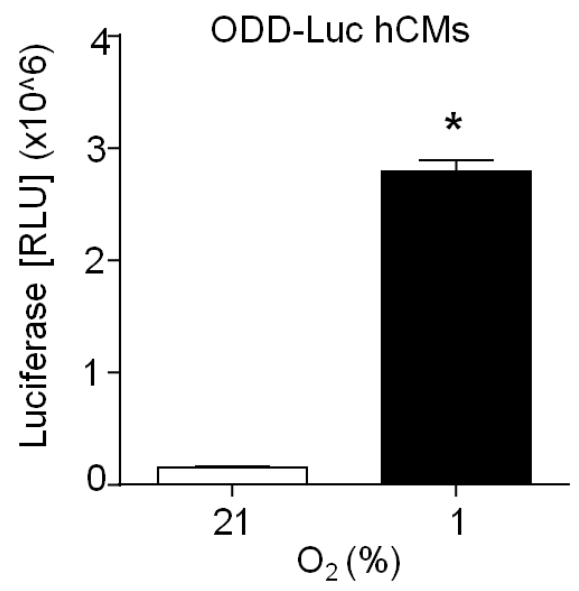

Figure 45. Hypoxia response in ODD-Luc human cardiomyocytes. Luc-signal development in ODD-Luc hCMs cultured in $21 \%$ and $1 \% \mathrm{O}_{2}$ for 4 hours ( $\mathrm{n}=3$ /group, unpaired $\mathrm{t}$-test, ${ }^{*} \mathrm{p}<0.05$ ). 


\section{Discussion}

Cardiac progenitor cells (CPCs) were identified in the heart based on Sca-1 and c-Kit surface markers (Beltrami et al. 2003, Oh et al. 2003). Despite some evidence for Sca-1-like cells in the human heart (Smits et al. 2009), c-Kit has been used as a common marker for CPCs in the human heart. The biological relevance of CPCs is under debate since their introduction more than 10 years ago (Beltrami et al. 2003) with several clinical trials being underway to identify the therapeutic utility of CPCs in the human heart (Bolli et al. 2011, Makkar et al. 2012). The interpretation of the biological relevance of CPCs in the heart is diverse, ranging from being transiently extravagated leukocytes or mast cells (Kubo et al. 2009) to being resident progenitors with strong cardiomyogenic activity (Ellison et al. 2013). Despite the ongoing clinical trials, phenotypic characterization and the perceived mode of action of CPCs are still under debate (Chong et al. 2014). The aim of this thesis was to make use of the EHM platform to scrutinize the biological activity of CPCs. The following specific hypotheses were tested:

1) CPCs support functional heart muscle formation in vitro.

2) CPCs elicit cardioprotective effects upon hypoxic injury.

The key results are summarized below:

1) CPCs are phenotypically distinct from fibroblasts.

2) Human CPCs contain a pericyte transcriptome signature.

3) CPCs and fibroblasts show a similar function as to the support of EHM formation.

4) Paracrine activity of CPCs can be enhanced by enforced miR-133a expression.

5) No evidence for CPC transdifferentiation into cardiomyocytes could be obtained.

6) Different EHM models of hypoxic damage could be established. 
7) Fibroblats, but not CPCs protected from hypoxia/reoxygenation injury.

8) CPCs protected EHM under chronic hypoxia.

9) Novel tools for imaging of cardiomyocyte oxygenation could be established.

\subsection{Characterization of CPCs}

Phenotypic characterization of c-Kit-CPCs isolated from adult mouse and human heart revealed that they do not express c-Kit, although they were isolated based on c-Kit expression. It is likely that the CPCs lost their c-Kit expression in culture over time, as observed in muscle-derived stem cells, which change their phenotype immediately after being isolated (Jankowski et al. 2001). Different types of CPCs have been characterized by expression of different cell surface markers, so far. Whether these CPCs represent unique cell types or are related to each other is still under debate (Torella et al. 2008). PDGFRa ${ }^{+} / \mathrm{Sca}-1^{+}$mesenchymal stem cell (MSC)like cells have been reported to exist in the adult mouse myocardium, with the potential to differentiate into three cardiac lineages. These cells were suggested to originate from epicardium and express c-Kit in a small subset (Chong et al. 2011). In our study, $\sim 90 \%$ of mouse c-Kit-CPCs were found to be PDGFRa ${ }^{+} / \mathrm{Sca}-1^{+}$as well as

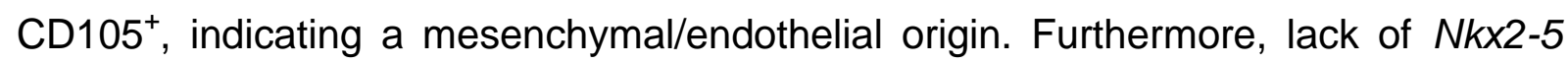
expression, as an early cardiac marker suggested that these CPCs were not committed to the cardiomyocyte lineage. Conversely, $\mathrm{PDGFRa}{ }^{+} / \mathrm{Sca}-1^{+}$cells have been related to epicardial progenitors, which are mainly involved in the constitution of the vascular compartments of the developing heart, including smooth muscle cells and pericytes (Kennedy-Lydon and Rosenthal 2015).

Pericytes are multipotential MSC-like cells that are positive for several distinct markers (MCAM, CSPG4, PDGFR,$\alpha-S M A$ ) as well as more broadly expressed mesenchymal cell markers including CD90, CD73, CD105, CD44 and can apparently be induced to differentiate into cardiomyocytes and smooth muscle cells (Chen et al. 2013, Crisan et al. 2008). Interestingly, human CPCs (hCPCs) showed a marked gene expression of MCAM, CSPG4 and PDGFRß, suggesting a pericyte-like 
phenotype. However, detection of PECAM1 expression in hCPCs, although at a low abundance, would not be fully compatible with this hypothesis (Chen et al. 2013).

Adventitial cells are another type of MSC-like progenitors expressing the endothelial/hematopoietic progenitor marker CD34 and mesenchymal cell markers (e.g. CD90, CD73, CD105 and CD44) with a potential to differentiate into endothelial-like and smooth muscle cells (Crisan et al. 2012). hCPCs did not express CD34. However, when compared to other cell types, hCPCs demonstrated an elevated expression of GLI1, which has recently been shown as a new marker to identify perivascular progenitors in the pericyte niche (Kramann et al. 2015). Although these cells are supposed to be negative for pericyte markers, they can indeed gain expression of pericyte-specific and smooth muscle cell-specific phenotypes (e.g. $\alpha$ $\mathrm{SMA}^{+}$) depending on in vitro and in vivo conditions (Kramann et al. 2015). In this respect, if hCPCs are true $\mathrm{GLI} 1^{+}$perivascular progenitors, this may explain the reason why they seem to show a high heterogeneous expression profile in vitro.

Mesoangioblasts resemble another blood vessel associated stem cell type, with apparent multipotential characteristics (Crisan et al. 2008). These cells can show heterogeneous characteristics with partial expression of endothelial cell, pericyte, perivascular progenitor markers, as well as c-Kit. Consistent with the heterogeneous gene expression profile in hCPCs, cardiac mesoangioblasts might be another candidate in describing the lineage identity of hCPCs.

Taken together, although each progenitor is defined by a combination of certain markers, these markers seem to be shared between different cell types (pericytes, adventitial cells and mesoangioblasts) suggesting that they may actually represent different intermediate forms of the same stem cell population in the heart. In this perspective, it is difficult to put CPCs under a specific class of stem cell population defined, so far. However, our phenotypic analysis suggests that they match closely with progenitor cells identified to be localized in perivascular niches. 


\subsection{Human CPCs might have an epicardial origin}

c-Kit ${ }^{+}$cells were identified to be located predominantly in the subepicardium of the right atria and adjacent to myocardial intersititium in the heart (Chong et al. 2013, Keith and Bolli 2015, Limana et al. 2007, Limana et al. 2010). These subepicardially located $\mathrm{C}-\mathrm{Kit}^{+}$cells are believed to be epicardial progenitors expressing epicardial developmental genes (e.g. Wt1 and Tbx18) during embryonic heart development and myocardial infarction (Limana et al. 2010). Given the fact that hCPCs in our study were originally isolated from the right atrial appendage, it is likely that they might be representing a subgroup of in vivo ${\mathrm{c}-\mathrm{Kit}^{+}}^{+}$progenitors subepicardially located in the right atria of the adult human heart.

In addition, epicardial progenitors are normally derived by a process called "epithelial to mesenchymal transformation" (EMT), whereby they develop a mesenchymal phenotype (Keith and Bolli 2015). In our study, we showed that not all, but a certain fraction of hCPCs present expression of mesenchymal markers; $36 \% \mathrm{CD}^{+}$and $60 \% \mathrm{CD} 105^{+}$, suggesting conversion to a mesenchymal transition state. Considering that the c-Kit receptor plays an important role in cell survival, migration and proliferation (Lennartsson and Ronnstrand 2012), c-Kit expression might have enabled progenitor cells to actively migrate towards the myocardium and gain an intermediate phenotype during the EMT process. Epicardial progenitors that undergo EMT highly contribute to vascular and interstitium compartments with little evidence of cardiomyocyte differentiation (Keith and Bolli 2015). In line with this, hCPCs did not show any late (cardiac actin and cardiac troponin I) or early cardiac marker (Nkx2-5) expression, but only enhanced GATA4. Furthermore, a small fraction $(10 \%)$ of hCPCs were PECAM1+ as an indication for their vasculogenic potential. Moreover, relatively higher expression of EMT associated proteins; periostin and TCF21 in hCPCs can be considered supporting evidence for a epicardial origin. Considering the role of epicardium in the development of vascular compartment in the heart, it is likely that hCPCs may represent a subgroup of vascular associated progenitors migrated from the epicardium and localized in tunica adventitia.

On the other hand, as demonstrated by gene ontology analysis of differentially expressed genes, hCPCs were found to express a number of genes related to cell 
adhesion, actin cytoskeleton organization, thereby cell migration (Appendix A5; e.g. Ras homology [Rho] GTPase family members RhoGTPase activating and RhoGTPase target genes as well as talin 1 [TLN1]). Considering the fact that $\mathrm{C}-\mathrm{Kit}^{+}$ epicardial progenitors are highly invasive migrating from the epicardium to mesoethelial layer during EMT process (Keith and Bolli 2015), it can be speculated that these hCPCs might have been derived from these epicardial progenitors preserving their intrinsic behavior and migration capacity. However, it should be also noted that hCPCs might be representing only a group of perivascular cells, which tend to cluster in oxygen-rich surface regions in EHM resembling their in vivo stem cell niche environment.

\subsection{CPCs and fibroblasts support EHM formation}

We demonstrated that mouse and human CPCs are capable of supporting functional heart muscle formation in vitro similar to fibroblasts, although they seemed to not support functional development optimally as indicated by lower contractile performance in CPC-EHM vs. fibroblast-EHM. Biochemical (e.g. hormones and growth factors) and biophysical cues (e.g. mechanical loading, substrate stiffness and isotropy) play an important role in mediating cardiac function (Liaw and Zimmermann 2016). Biochemical factors including triiodothyronine (T3), insulin, growth hormone and IGF-1 as an activator of PI3K/Akt signaling pathway are among the particular factors involved in physiological hypertrophy and calcium homeostasis (Louch et al. 2015). As demonstrated from RNAseq data, hCPCs seem to have a different secretome profile than fibroblasts. One interesting observation, which could be related to the lower contractile performance in CPC-EHM, was lower IGF-1 expression by hCPCs. In previous studies, we observed that insulin and IGF-1 can enhance contractile performance of EHM (Zimmermann et al. 2002); with both effects likely mediated via the IGF-1 receptor. Also the data on CPC optimization by enforced miR133-a expression, in the mouse model, suggested a important involvement of IGF-1 in EHM functionality and cardimoyctye survival (Izarra et al. 2014). Thus one may conclude that CPCs have a limited paracrine activity under basal conditions, which can be enhanced by genetic manipulation to achieve optimal heart muscle support. Whether similar genetic modifications would also enhance the biololgical activity of other cell types, such as fibroblasts, remains to be investigated. 


\subsection{CPCs do not contribute to cardiac homeostasis under normal conditions}

Isolated CPCs require special microenvironmental conditions for the induction of cardiomyocyte differentiation in vitro, such as stimulation with chemicals including dexamethasone (Beltrami et al. 2003) and 5-azacytidine (Oh et al. 2003), growth factors (Goumans et al. 2007) as well as co-culturing with cardiomyocytes (Bearzi et al. 2007). However, hCPCs were not detected to differentiate into cardiomyocytes in the EHM co-culture model, as demonstrated by lack of sarcomeric a-actinin expression in GFP-labelled CPCs. This finding was in contrary to other studies describing myogenic specification of CPCs co-cultured with adult cardiomyocytes (Pfister et al. 2005). Obvious reasons for the failure to detect cardiomyocyte transdifferentiation could be: (1) hESC-derived cardiomyocytes and hFFs may not support CPC transdifferentiation; (2) hCPCs do not possess a cardiogenic potential.

Cardiosphere-derived progenitors have been reported to enhance cell cycle activity of endogenous cardiomyocytes when injected into the myocardium after myocardial infarction (Malliaras et al. 2013). In addition, delivery of C-Kit ${ }^{+}$CPCs into the infarcted myocardium promoted the formation of proliferative new cardiomyocytes in long-term (Tang et al. 2016). According to these published evidences, one of the mechanisms underlying cardio-regenerative effect of CPCs seems to work through induction of proliferation of endogenous cardiomyocytes. In contrast to this proposed mechanism, we did not detect any additional proliferating response in cardiomyocytes co-cultured with hCPCs.

\subsection{CPCs can be modified to enhance their tissue-supporting activity}

Low cell retention and engraftment are limiting factors that weaken the success of a cell-based therapy. Genetic modification is one of the key approaches to enhance delivery efficacy of transplanted cells (Terrovitis et al. 2010). Survival of CPCs after injection into the infarcted myocardium was studied by our collaborators (CARE-MI FP7 Consortium; Antonio Bernad; (Izarra, 2014 \#95). Overexpression of miR133a in CPC enhanced their therapeutic efficacy in vivo. These observations were in line with reports on the ciritical role of mir-133a in stimulating cardiomyocyte proliferation during heart development (Meder et al. 2008). Unexpectedly, enforced mir-133a 
expression did not increase CPC engraftment in vivo. In agreement with the enhanced therapeutic efficacy in vivo we observed an increased paracrine activity of mir-133a-CPCs in rat EHM. The observed reduced cardiomyocyte apoptosis in CPCmiR133a supplemented EHM could have accounted for the improved functional outcome in EHM, but also in the in vivo experiments. These experiments demonstrate the utility of the EHM culture format in defining the mode of action in potential cell therapeutics. Whether the specific modification tested would only be effective in CPCs remain to be studied.

\subsection{Development of EHM models of hypoxic damage}

Heart muscle can undergo a temporal dysfunction, so called myocardial stunning or be permanently damaged leading to lethal ischemia/reperfusion injury. In addition, response to ischemia/reperfusion injury can also depend on the cell type and organ (Kalogeris et al. 2012). Although the exact mechanism that shifts from reversible ischemia to irreversible ischemia/reperfusion injury is not well-known, it most likely requires a number biochemical and metabolic changes including loss of a critical amount of ATP and $\mathrm{Ca}^{2+}$ overload (Rovetto et al. 1975). To develop a model system for ischemic heart damage, we tested different hypoxia and reoxygenation protocols in EHM culture. 72 hrs of hypoxia resulted in high lactate accumulation, significant ATP depletion and loss of HIF-1 activity in monolayer cardiomyocytes. In EHM culture, contractile performance was not obviously disturbed after $72 \mathrm{hrs}$ of hypoxia, but clearly diminished after additional $24 \mathrm{hrs}$ of reoxygenation. In addition to reduced basal force of contraction, high calcium sensitivity (suggesting calcium overload) and less $\beta$-adrenergic response was observed. Extended hypoxia over $120 \mathrm{hrs}$ resulted in similar damage as observed in the $72 \mathrm{hrs}$ hypoxia $24 \mathrm{hrs}$ reoxygenation experiment. These data identify a narrow time window for the simulation of hypoxia/reperfusion damage in EHM. An unanticipated observation was that EHM subjected to hypoxia/reoxygenation injury could be partially protected from fibroblasts, but not CPC-conditioned medium, suggesting a protective paracrine activity in fibroblasts.

RNAseq profiling identified enhanced anti-apoptotic and cell proliferation/growth enhancing effects in hFFs, which may explain the observed differential effect of conditions medium from hCPCs and hFFs. The suggested anti-inflammatory and 
angiogenesis inducing activity in hCPCs may, in the absence of the respective target cells, not be functional in EHM. These data collectively suggest that complex multicellular models are needed to fully determine the biological activity of CPCs. Finally, our data supports the use of animal models to fully determine the likely comprehensive effects of cell based therapies.

\subsection{CPCs protect EHM under chronic hypoxia}

Cardiac progenitor cell based therapy, regardless of the delivery mode, requires the deposition and retention (at least transiently) of CPCs in or adjacent to damaged myocardium, which is typically inflamed and hypoxic ( $\mathrm{Li}$ et al. 2016). In agreement with the concept of cell context specific efficacy of CPCs, we developed EHM with the most abundant cardiac cell populations, i.e., cardiomyocyte and fibroblasts, supplemented with CPCs. Finally, these EHM tri-cultures were composed of $65 \%$ cardiomyocyte, $28 \%$ foreskin fibroblasts and $7 \%$ hCPCs to simulate a native heart cell composition. Interestingly, EHM tri-cultures were protected from chronic (120 hrs) hypoxic injury. In line with the finding in H/R injury model, hCPCs did not enhance survival of cardiomyocytes, but helped to maintain strucutural and functional integrity, suggesting that hCPCs played a critical role in the adaptation to chronic hypoxia. Thus far, we propose that CPCs under chronic hypoxia support a myocardial hibernation state (Holley et al. 2015). The underlying mechanisms, especially with respect to mitochondria function and metabolism, need to be studied in more detail. Whether a modification of hCPCs, such as enforced miR133a expression, would enhance the therapeutic efficacy as to a protection from cell death remains to be studied.

\subsection{Live cell imaging of cardiomyocyte oxygenation}

To gain further insight into the mechanisms underlying hypoxic damage and protection from hypoxia, including hypoxia/reoxygenation injury, it is important to on the one hand establish a human model and on the other hand visualize cardiomyocyte specific oxygenation. We first demonstrated that a recently established hypoxia sensor, ODD-Luc can be integrated and utilized to visualize and identify the endogenous biological response to hypoxia in mouse embryonic stem 
cell-derived cardiomyocytes (Hesse et al. 2014). Having the first evidence of applicability of ODD-Luc cardiomyocytes for imaging hypoxia in tissue level in a mouse EHM model, we next developed a human model that involves a human embryonic stem cell (hESC) line genetically modified to express ODD-Luc fusion protein. The preliminary data in human system first showed that cardiomyocytes differentiated from hESCs successfully express ODD-Luc with protein stabilization and thus enhanced Luc-signal under hypoxia. This indicates that it should be feasible to develop a human ODD-Luc EHM model for more detailed studies of the mechanisms underlying hypoxia associated myocardial damage as well as protection from hypoxic injury. 


\section{Conclusion and Perspectives}

Engineered Heart Muscle (EHM) was established as model system for the evaluation of the biological activity of cardiac progenitor cells (CPCs) from mouse and human. Color-coding of cardiomyocytes and non-myocytes in EHM allowed for cell type specific morphological and molecular analyses. Interestingly, fibroblasts and CPCs, despite being distinct cell entities based on their molecular properties, supported functional assembly of EHM in a similar way. Moreover, fibroblasts, but not CPCs, appear to be protective in a $\mathrm{H} / \mathrm{R}$ injury model. Conversely, CPCs were critical and supportive for the maintanence of cardiac function in EHM subjected to chronic hypoxia. This effect in the presence of cardiomyocytes and fibroblasts appeared cell context dependent. Genetic manipulation of CPCs by forced expression of miR-133a was established as a model system for enhanced biological activity. The ODD-Luc model will be instrumental not only for in vitro studies, but also to trace EHM survival after implantation in vivo. 


\section{Bibliography}

1. Achilli F, Malafronte C, Lenatti L, Gentile F, Dadone V, Gibelli G, Maggiolini S, Squadroni L, Di Leo C, Burba I, Pesce M, Mircoli L, Capogrossi MC, Di Lelio A, Camisasca P, Morabito A, Colombo G, Pompilio G (2010) Granulocyte colonystimulating factor attenuates left ventricular remodelling after acute anterior STEMI: results of the single-blind, randomized, placebo-controlled multicentre STem cEll Mobilization in Acute Myocardial Infarction (STEM-AMI) Trial. Eur $J$ Heart Fail. 12:1111-21.

2. Ali SR, Hippenmeyer S, Saadat LV, Luo L, Weissman IL, Ardehali R (2014) Existing cardiomyocytes generate cardiomyocytes at a low rate after birth in mice. Proc Natl Acad Sci U S A. 111:8850-5.

3. Assmus B, Fischer-Rasokat U, Honold J, Seeger FH, Fichtlscherer S, Tonn T, Seifried E, Schachinger V, Dimmeler S, Zeiher AM (2007) Transcoronary transplantation of functionally competent BMCs is associated with a decrease in natriuretic peptide serum levels and improved survival of patients with chronic postinfarction heart failure: results of the TOPCARE-CHD Registry. Circ Res. 100:1234-41.

4. Bartunek J, Behfar A, Dolatabadi D, Vanderheyden M, Ostojic M, Dens J, El Nakadi B, Banovic M, Beleslin B, Vrolix M, Legrand V, Vrints C, Vanoverschelde JL, Crespo-Diaz R, Homsy C, Tendera M, Waldman S, Wijns W, Terzic A (2013) Cardiopoietic stem cell therapy in heart failure: the C-CURE (Cardiopoietic stem Cell therapy in heart failURE) multicenter randomized trial with lineage-specified biologics. J Am Coll Cardiol. 61:2329-38.

5. Bearzi C, Rota M, Hosoda T, Tillmanns J, Nascimbene A, De Angelis A, Yasuzawa-Amano S, Trofimova I, Siggins RW, Lecapitaine N, Cascapera S, Beltrami AP, D'Alessandro DA, Zias E, Quaini F, Urbanek K, Michler RE, Bolli R, Kajstura J, Leri A, Anversa P (2007) Human cardiac stem cells. Proc Natl Acad Sci U S A. 104:14068-73. 
6. Beltrami AP, Barlucchi L, Torella D, Baker M, Limana F, Chimenti S, Kasahara H, Rota M, Musso E, Urbanek K, Leri A, Kajstura J, Nadal-Ginard B, Anversa P (2003) Adult cardiac stem cells are multipotent and support myocardial regeneration. Cell. 114:763-76.

7. Bergmann O, Bhardwaj RD, Bernard S, Zdunek S, Barnabe-Heider F, Walsh S, Zupicich J, Alkass K, Buchholz BA, Druid H, Jovinge S, Frisen J (2009) Evidence for cardiomyocyte renewal in humans. Science. 324:98-102.

8. Bergmann $\mathrm{O}$, Zdunek S, Felker A, Salehpour M, Alkass K, Bernard S, Sjostrom SL, Szewczykowska M, Jackowska T, Dos Remedios C, Malm T, Andra M, Jashari R, Nyengaard JR, Possnert G, Jovinge S, Druid H, Frisen J (2015) Dynamics of Cell Generation and Turnover in the Human Heart. Cell. 161:1566-75.

9. Bernex F, De Sepulveda P, Kress C, Elbaz C, Delouis C, Panthier JJ (1996) Spatial and temporal patterns of c-kit-expressing cells in WlacZ/+ and WlacZ/WlacZ mouse embryos. Development. 122:3023-33.

10. Bersell K, Arab S, Haring B, Kuhn B (2009) Neuregulin1/ErbB4 Signaling Induces Cardiomyocyte Proliferation and Repair of Heart Injury. Cell. 138:257-270.

11. Bolli R, Chugh AR, D'Amario D, Loughran JH, Stoddard MF, Ikram S, Beache GM, Wagner SG, Leri A, Hosoda T, Sanada F, Elmore JB, Goichberg P, Cappetta D, Solankhi NK, Fahsah I, Rokosh DG, Slaughter MS, Kajstura J, Anversa P (2011) Cardiac stem cells in patients with ischaemic cardiomyopathy (SCIPIO): initial results of a randomised phase 1 trial. Lancet. 378:1847-57.

12. Bolli R, Tang XL, Sanganalmath SK, Rimoldi O, Mosna F, Abdel-Latif A, Jneid H, Rota M, Leri A, Kajstura J (2013) Intracoronary delivery of autologous cardiac stem cells improves cardiac function in a porcine model of chronic ischemic cardiomyopathy. Circulation. 128:122-31.

13. Chen CW, Okada M, Proto JD, Gao X, Sekiya N, Beckman SA, Corselli M, Crisan M, Saparov A, Tobita K, Peault B, Huard J (2013) Human pericytes for ischemic heart repair. Stem Cells. 31:305-16. 
14. Chimenti I, Smith RR, Li TS, Gerstenblith G, Messina E, Giacomello A, Marban E (2010) Relative roles of direct regeneration versus paracrine effects of human cardiosphere-derived cells transplanted into infarcted mice. Circ Res. 106:971-80.

15. Cho HJ, Lee HJ, Song MK, Seo JY, Bae YH, Kim JY, Lee HY, Lee W, Koo BK, Oh BH, Park YB, Kim HS (2013) Vascular calcifying progenitor cells possess bidirectional differentiation potentials. PLoS Biol. 11:e1001534.

16. Chong JJ, Chandrakanthan V, Xaymardan M, Asli NS, Li J, Ahmed I, Heffernan C, Menon MK, Scarlett CJ, Rashidianfar A, Biben C, Zoellner H, Colvin EK, Pimanda JE, Biankin AV, Zhou B, Pu WT, Prall OW, Harvey RP (2011) Adult cardiac-resident MSC-like stem cells with a proepicardial origin. Cell Stem Cell. 9:527-40.

17. Chong JJ, Reinecke H, Iwata M, Torok-Storb B, Stempien-Otero A, Murry CE (2013) Progenitor cells identified by PDGFR-alpha expression in the developing and diseased human heart. Stem Cells Dev. 22:1932-43.

18. Chong JJ, Forte E, Harvey RP (2014) Developmental origins and lineage descendants of endogenous adult cardiac progenitor cells. Stem Cell Res. 13:592614.

19. Christalla P, Hudson JE, Zimmermann W-H (2012) The Cardiogenic Niche as a Fundamental Building Block of Engineered Myocardium. Cells Tissues Organs. 195:82-93.

20. Chugh AR, Beache GM, Loughran JH, Mewton N, Elmore JB, Kajstura J, Pappas P, Tatooles A, Stoddard MF, Lima JA, Slaughter MS, Anversa P, Bolli R (2012) Administration of cardiac stem cells in patients with ischemic cardiomyopathy: the SCIPIO trial: surgical aspects and interim analysis of myocardial function and viability by magnetic resonance. Circulation. 126:S54-64.

21. Crisan M, Yap S, Casteilla L, Chen CW, Corselli M, Park TS, Andriolo G, Sun B, Zheng B, Zhang L, Norotte C, Teng PN, Traas J, Schugar R, Deasy BM, Badylak S, Buhring HJ, Giacobino JP, Lazzari L, Huard J, Peault B (2008) A perivascular 
origin for mesenchymal stem cells in multiple human organs. Cell Stem Cell. 3:30113.

22. Crisan M, Corselli M, Chen WC, Peault B (2012) Perivascular cells for regenerative medicine. J Cell Mol Med. 16:2851-60.

23. Davis DR, Zhang Y, Smith RR, Cheng K, Terrovitis J, Malliaras K, Li TS, White A, Makkar R, Marban E (2009) Validation of the cardiosphere method to culture cardiac progenitor cells from myocardial tissue. PLoS One. 4:e7195.

24. Duff SE, Li C, Garland JM, Kumar S (2003) CD105 is important for angiogenesis: evidence and potential applications. FASEB J. 17:984-92.

25. Ellison Georgina M, Vicinanza C, Smith Andrew J, Aquila I, Leone A, Waring Cheryl D, Henning Beverley J, Stirparo Giuliano G, Papait R, Scarfò M, Agosti V, Viglietto G, Condorelli G, Indolfi C, Ottolenghi S, Torella D, Nadal-Ginard B (2013) Adult c-kitpos Cardiac Stem Cells Are Necessary and Sufficient for Functional Cardiac Regeneration and Repair. Cell. 154:827-842.

26. Engel FB, Hsieh PCH, Lee RT, Keating MT (2006) FGF1/p38 MAP kinase inhibitor therapy induces cardiomyocyte mitosis, reduces scarring, and rescues function after myocardial infarction. P Natl Acad Sci USA. 103:15546-15551.

27. Ferreira-Martins J, Ogorek B, Cappetta D, Matsuda A, Signore S, D'Amario D, Kostyla J, Steadman E, Ide-Iwata N, Sanada F, laffaldano G, Ottolenghi S, Hosoda T, Leri A, Kajstura J, Anversa P, Rota M (2012) Cardiomyogenesis in the developing heart is regulated by c-kit-positive cardiac stem cells. Circ Res. 110:701-15.

28. Fischer KM, Cottage CT, Wu W, Din S, Gude NA, Avitabile D, Quijada P, Collins BL, Fransioli J, Sussman MA (2009) Enhancement of myocardial regeneration through genetic engineering of cardiac progenitor cells expressing Pim1 kinase. Circulation. 120:2077-87.

29. Freire AG, Nascimento DS, Forte G, Valente M, Resende TP, Pagliari S, Abreu C, Carvalho I, Di Nardo P, Pinto-do OP (2014) Stable phenotype and function of immortalized Lin-Sca-1+ cardiac progenitor cells in long-term culture: a step closer to standardization. Stem Cells Dev. 23:1012-26. 
30. Furtado MB, Costa MW, Pranoto EA, Salimova E, Pinto AR, Lam NT, Park A, Snider P, Chandran A, Harvey RP, Boyd R, Conway SJ, Pearson J, Kaye DM, Rosenthal NA (2014) Cardiogenic genes expressed in cardiac fibroblasts contribute to heart development and repair. Circ Res. 114:1422-34.

31. Gerbin KA, Murry CE (2015) The winding road to regenerating the human heart. Cardiovasc Pathol. 24:133-40.

32. Godier-Furnemont AF, Tiburcy M, Wagner E, Dewenter M, Lammle S, ElArmouche A, Lehnart SE, Vunjak-Novakovic G, Zimmermann WH (2015) Physiologic force-frequency response in engineered heart muscle by electromechanical stimulation. Biomaterials. 60:82-91.

33. Goumans MJ, de Boer TP, Smits AM, van Laake LW, van Vliet P, Metz $\mathrm{CH}$, Korfage TH, Kats KP, Hochstenbach R, Pasterkamp G, Verhaar MC, van der Heyden MA, de Kleijn D, Mummery CL, van Veen TA, Sluijter JP, Doevendans PA (2007) TGF-beta1 induces efficient differentiation of human cardiomyocyte progenitor cells into functional cardiomyocytes in vitro. Stem Cell Res. 1:138-49.

34. Hassink RJ, Pasumarthi KB, Nakajima $H$, Rubart $M$, Soonpaa $M H$, de la Riviere AB, Doevendans PA, Field LJ (2008) Cardiomyocyte cell cycle activation improves cardiac function after myocardial infarction. Cardiovascular Research. 78:18-25.

35. Hesse AR, Levent E, Zieseniss A, Tiburcy M, Zimmermann WH, Katschinski DM (2014) Lights on for HIF-1alpha: genetically enhanced mouse cardiomyocytes for heart tissue imaging. Cell Physiol Biochem. 34:455-62.

36. Holley CT, Long EK, Lindsey ME, McFalls EO, Kelly RF (2015) Recovery of hibernating myocardium: what is the role of surgical revascularization? J Card Surg. 30:224-31.

37. Houtgraaf $\mathrm{JH}$, den Dekker WK, van Dalen BM, Springeling $T$, de Jong $R$, van Geuns RJ, Geleijnse ML, Fernandez-Aviles F, Zijlsta F, Serruys PW, Duckers HJ (2012) First experience in humans using adipose tissue-derived regenerative cells in the treatment of patients with ST-segment elevation myocardial infarction. J Am Coll Cardiol. 59:539-40. 
38. Hsieh PCH, Segers VFM, Davis ME, MacGillivray C, Gannon J, Molkentin JD, Robbins J, Lee RT (2007) Evidence from a genetic fate-mapping study that stem cells refresh adult mammalian cardiomyocytes after injury. Nature Medicine. 13:970974.

39. Hu Y, Zhang Z, Torsney E, Afzal AR, Davison F, Metzler B, Xu Q (2004) Abundant progenitor cells in the adventitia contribute to atherosclerosis of vein grafts in ApoE-deficient mice. J Clin Invest. 113:1258-65.

40. Huang da W, Sherman BT, Lempicki RA (2009) Systematic and integrative analysis of large gene lists using DAVID bioinformatics resources. Nat Protoc. 4:4457.

41. Hudson J, Titmarsh D, Hidalgo A, Wolvetang E, Cooper-White J (2012) Primitive Cardiac Cells from Human Embryonic Stem Cells. Stem Cells and Development. 21:1513-1523.

42. Ikuta K, Weissman IL (1992) Evidence that hematopoietic stem cells express mouse c-kit but do not depend on steel factor for their generation. Proc Natl Acad Sci U S A. 89:1502-6.

43. Irion S, Luche H, Gadue P, Fehling HJ, Kennedy M, Keller G (2007) Identification and targeting of the ROSA26 locus in human embryonic stem cells. Nat Biotechnol. 25:1477-82.

44. Ito CY, Li CY, Bernstein A, Dick JE, Stanford WL (2003) Hematopoietic stem cell and progenitor defects in Sca-1/Ly-6A-null mice. Blood. 101:517-23.

45. Izarra A, Moscoso I, Levent E, Cañón S, Cerrada I, Díez-Juan A, Blanca V, Núñez-Gil I-J, Valiente I, Ruíz-Sauri A, Sepúlveda P, Tiburcy M, Zimmermann W-H, Bernad A (2014) miR-133a Enhances the Protective Capacity of Cardiac Progenitors Cells after Myocardial Infarction. Stem Cell Reports. 3:1029-1042.

46. Jankowski RJ, Haluszczak C, Trucco M, Huard J (2001) Flow cytometric characterization of myogenic cell populations obtained via the preplate technique: potential for rapid isolation of muscle-derived stem cells. Hum Gene Ther. 12:619-28. 
47. Kalogeris T, Baines CP, Krenz M, Korthuis RJ (2012) Cell biology of ischemia/reperfusion injury. Int Rev Cell Mol Biol. 298:229-317.

48. Keith MC, Bolli R (2015) "String theory" of c-kit(pos) cardiac cells: a new paradigm regarding the nature of these cells that may reconcile apparently discrepant results. Circ Res. 116:1216-30.

49. Kennedy-Lydon T, Rosenthal N (2015) Cardiac regeneration: epicardial mediated repair. Proc Biol Sci. 282.

50. Kim D, Pertea G, Trapnell C, Pimentel H, Kelley R, Salzberg SL (2013) TopHat2: accurate alignment of transcriptomes in the presence of insertions, deletions and gene fusions. Genome Biol. 14:R36.

51. Klug MG, Soonpaa MH, Koh GY, Field LJ (1996) Genetically selected cardiomyocytes from differentiating embronic stem cells form stable intracardiac grafts. J Clin Invest. 98:216-24.

52. Kovacic JC, Boehm M (2009) Resident vascular progenitor cells: an emerging role for non-terminally differentiated vessel-resident cells in vascular biology. Stem Cell Res. 2:2-15.

53. Kramann R, Schneider RK, DiRocco DP, Machado F, Fleig S, Bondzie PA, Henderson JM, Ebert BL, Humphreys BD (2015) Perivascular Gli1+ progenitors are key contributors to injury-induced organ fibrosis. Cell Stem Cell. 16:51-66.

54. Kubo H, Berretta RM, Jaleel N, Angert D, Houser SR (2009) c-Kit+ bone marrow stem cells differentiate into functional cardiac myocytes. Clin Transl Sci. 2:26-32.

55. Kuhn B, del Monte F, Hajjar RJ, Chang YS, Lebeche D, Arab S, Keating MT (2007) Periostin induces proliferation of differentiated cardiomyocytes and promotes cardiac repair. Nature Medicine. 13:962-969.

56. Laflamme MA, Murry CE (2011) Heart regeneration. Nature. 473:326-35. 
57. Lauden L, Boukouaci W, Borlado LR, Lopez IP, Sepulveda P, Tamouza R, Charron D, Al-Daccak R (2013) Allogenicity of human cardiac stem/progenitor cells orchestrated by programmed death ligand 1. Circ Res. 112:451-64.

58. Lennartsson J, Ronnstrand L (2012) Stem cell factor receptor/c-Kit: from basic science to clinical implications. Physiol Rev. 92:1619-49.

59. Li TS, Cheng K, Malliaras K, Smith RR, Zhang $Y$, Sun B, Matsushita N, Blusztajn A, Terrovitis J, Kusuoka H, Marban L, Marban E (2012) Direct comparison of different stem cell types and subpopulations reveals superior paracrine potency and myocardial repair efficacy with cardiosphere-derived cells. J Am Coll Cardiol. 59:942-53.

60. Li X, Tamama K, Xie X, Guan J (2016) Improving Cell Engraftment in Cardiac Stem Cell Therapy. Stem Cells Int. 2016:7168797.

61. Liang J, Wu YL, Chen BJ, Zhang W, Tanaka Y, Sugiyama H (2013) The C-kit receptor-mediated signal transduction and tumor-related diseases. Int $J$ Biol Sci. 9:435-43.

62. Liaw NY, Zimmermann WH (2016) Mechanical stimulation in the engineering of heart muscle. Adv Drug Deliv Rev. 96:156-60.

63. Limana F, Zacheo A, Mocini D, Mangoni A, Borsellino G, Diamantini A, De Mori R, Battistini L, Vigna E, Santini M, Loiaconi V, Pompilio G, Germani A, Capogrossi MC (2007) Identification of myocardial and vascular precursor cells in human and mouse epicardium. Circ Res. 101:1255-65.

64. Limana F, Bertolami C, Mangoni A, Di Carlo A, Avitabile D, Mocini D, lannelli P, De Mori R, Marchetti C, Pozzoli O, Gentili C, Zacheo A, Germani A, Capogrossi MC (2010) Myocardial infarction induces embryonic reprogramming of epicardial ckit(+) cells: role of the pericardial fluid. J Mol Cell Cardiol. 48:609-18.

65. Liu ML, Nagai T, Tokunaga M, Iwanaga K, Matsuura K, Takahashi T, Kanda M, Kondo N, Naito AT, Komuro I, Kobayashi Y (2014) Anti-inflammatory peptides from cardiac progenitors ameliorate dysfunction after myocardial infarction. $J A m$ Heart Assoc. 3:e001101. 
66. Livak KJ, Schmittgen TD (2001) Analysis of relative gene expression data using real-time quantitative PCR and the 2(-Delta Delta $\mathrm{C}(\mathrm{T})$ ) Method. Methods. 25:402-8.

67. Louch WE, Koivumaki JT, Tavi P (2015) Calcium signalling in developing cardiomyocytes: implications for model systems and disease. J Physiol. 593:1047-63.

68. Lunde $\mathrm{K}$, Solheim $\mathrm{S}$, Aakhus $\mathrm{S}$, Arnesen $\mathrm{H}$, Abdelnoor $\mathrm{M}$, Egeland $\mathrm{T}$, Endresen K, llebekk A, Mangschau A, Fjeld JG, Smith HJ, Taraldsrud E, Grogaard HK, Bjornerheim R, Brekke M, Muller C, Hopp E, Ragnarsson A, Brinchmann JE, Forfang K (2006) Intracoronary injection of mononuclear bone marrow cells in acute myocardial infarction. N Engl J Med. 355:1199-209.

69. Makkar RR, Smith RR, Cheng K, Malliaras K, Thomson LE, Berman D, Czer LS, Marban L, Mendizabal A, Johnston PV, Russell SD, Schuleri KH, Lardo AC, Gerstenblith G, Marban E (2012) Intracoronary cardiosphere-derived cells for heart regeneration after myocardial infarction (CADUCEUS): a prospective, randomised phase 1 trial. Lancet. 379:895-904.

70. Malliaras K, Zhang Y, Seinfeld J, Galang G, Tseliou E, Cheng K, Sun B, Aminzadeh M, Marban E (2013) Cardiomyocyte proliferation and progenitor cell recruitment underlie therapeutic regeneration after myocardial infarction in the adult mouse heart. EMBO Mol Med. 5:191-209.

71. Martin CM, Meeson AP, Robertson SM, Hawke TJ, Richardson JA, Bates S, Goetsch SC, Gallardo TD, Garry DJ (2004) Persistent expression of the ATP-binding cassette transporter, Abcg2, identifies cardiac SP cells in the developing and adult heart. Dev Biol. 265:262-75.

72. Matsuura K, Nagai T, Nishigaki N, Oyama T, Nishi J, Wada H, Sano M, Toko H, Akazawa H, Sato T, Nakaya H, Kasanuki H, Komuro I (2004) Adult cardiac Sca-1positive cells differentiate into beating cardiomyocytes. J Biol Chem. 279:11384-91.

73. Meder B, Katus HA, Rottbauer W (2008) Right into the heart of microRNA133a. Genes Dev. 22:3227-31. 
74. Menasche $\mathrm{P}$, Alfieri $\mathrm{O}$, Janssens $\mathrm{S}$, McKenna $\mathrm{W}$, Reichenspurner $\mathrm{H}$, Trinquart L, Vilquin JT, Marolleau JP, Seymour B, Larghero J, Lake S, Chatellier G, Solomon S, Desnos M, Hagege AA (2008) The Myoblast Autologous Grafting in Ischemic Cardiomyopathy (MAGIC) trial: first randomized placebo-controlled study of myoblast transplantation. Circulation. 117:1189-200.

75. Messina E, De Angelis L, Frati G, Morrone S, Chimenti S, Fiordaliso F, Salio M, Battaglia M, Latronico MV, Coletta M, Vivarelli E, Frati L, Cossu G, Giacomello A (2004) Isolation and expansion of adult cardiac stem cells from human and murine heart. Circ Res. 95:911-21.

76. Meyer GP, Wollert KC, Lotz J, Pirr J, Rager U, Lippolt P, Hahn A, Fichtner S, Schaefer A, Arseniev L, Ganser A, Drexler H (2009) Intracoronary bone marrow cell transfer after myocardial infarction: 5-year follow-up from the randomized-controlled BOOST trial. Eur Heart J. 30:2978-84.

77. Mill JG, Stefanon I, dos Santos L, Baldo MP (2011) Remodeling in the ischemic heart: the stepwise progression for heart. Brazilian Journal of Medical and Biological Research. 44:890-898.

78. Miura M, Miura Y, Padilla-Nash HM, Molinolo AA, Fu B, Patel V, Seo BM, Sonoyama W, Zheng JJ, Baker CC, Chen W, Ried T, Shi S (2006) Accumulated chromosomal instability in murine bone marrow mesenchymal stem cells leads to malignant transformation. Stem Cells. 24:1095-103.

79. Miyamoto S, Kawaguchi N, Ellison GM, Matsuoka R, Shin'oka T, Kurosawa H (2010) Characterization of long-term cultured c-kit+ cardiac stem cells derived from adult rat hearts. Stem Cells Dev. 19:105-16.

80. Moroz E, Carlin S, Dyomina K, Burke S, Thaler HT, Blasberg R, Serganova I (2009) Real-time imaging of HIF-1alpha stabilization and degradation. PLoS One. 4:e5077.

81. Mushtaq M, DiFede DL, Golpanian S, Khan A, Gomes SA, Mendizabal A, Heldman AW, Hare JM (2014) Rationale and design of the Percutaneous Stem Cell Injection Delivery Effects on Neomyogenesis in Dilated Cardiomyopathy (the POSEIDON-DCM study): a phase $1 / \mathrm{II}$, randomized pilot study of the comparative 
safety and efficacy of transendocardial injection of autologous mesenchymal stem cell vs. allogeneic mesenchymal stem cells in patients with non-ischemic dilated cardiomyopathy. J Cardiovasc Transl Res. 7:769-80.

82. Nagy A, Rossant J, Nagy R, Abramow-Newerly W, Roder JC (1993) Derivation of completely cell culture-derived mice from early-passage embryonic stem cells. Proc Natl Acad Sci U S A. 90:8424-8.

83. Naito H, Melnychenko I, Didie M, Schneiderbanger K, Schubert P, Rosenkranz $\mathrm{S}$, Eschenhagen T, Zimmermann WH (2006) Optimizing engineered heart tissue for therapeutic applications as surrogate heart muscle. Circulation. 114:I72-8.

84. Noack C, Zafiriou MP, Schaeffer HJ, Renger A, Pavlova E, Dietz R, Zimmermann WH, Bergmann MW, Zelarayan LC (2012) Krueppel-like factor 15 regulates Wnt/beta-catenin transcription and controls cardiac progenitor cell fate in the postnatal heart. EMBO Mol Med. 4:992-1007.

85. Noseda M, Harada M, McSweeney S, Leja T, Belian E, Stuckey DJ, Abreu Paiva MS, Habib J, Macaulay I, de Smith AJ, al-Beidh F, Sampson R, Lumbers RT, Rao P, Harding SE, Blakemore AI, Jacobsen SE, Barahona M, Schneider MD (2015) PDGFRalpha demarcates the cardiogenic clonogenic Sca1+ stem/progenitor cell in adult murine myocardium. Nat Commun. 6:6930.

86. Oh H, Bradfute SB, Gallardo TD, Nakamura T, Gaussin V, Mishina Y, Pocius J, Michael LH, Behringer RR, Garry DJ, Entman ML, Schneider MD (2003) Cardiac progenitor cells from adult myocardium: homing, differentiation, and fusion after infarction. Proc Natl Acad Sci U S A. 100:12313-8.

87. Pavo N, Charwat S, Nyolczas N, Jakab A, Murlasits Z, Bergler-Klein J, Nikfardjam M, Benedek I, Benedek T, Pavo IJ, Gersh BJ, Huber K, Maurer G, Gyongyosi M (2014) Cell therapy for human ischemic heart diseases: critical review and summary of the clinical experiences. J Mol Cell Cardiol. 75:12-24.

88. Perin EC, Willerson JT, Pepine CJ, Henry TD, Ellis SG, Zhao DX, Silva GV, Lai D, Thomas JD, Kronenberg MW, Martin AD, Anderson RD, Traverse JH, Penn MS, Anwaruddin S, Hatzopoulos AK, Gee AP, Taylor DA, Cogle CR, Smith D, Westbrook L, Chen J, Handberg E, Olson RE, Geither C, Bowman S, Francescon J, 
Baraniuk S, Piller LB, Simpson LM, Loghin C, Aguilar D, Richman S, Zierold C, Bettencourt J, Sayre SL, Vojvodic RW, Skarlatos SI, Gordon DJ, Ebert RF, Kwak M, Moye LA, Simari RD (2012) Effect of transendocardial delivery of autologous bone marrow mononuclear cells on functional capacity, left ventricular function, and perfusion in chronic heart failure: the FOCUS-CCTRN trial. JAMA. 307:1717-26.

89. Pfister O, Mouquet F, Jain M, Summer R, Helmes M, Fine A, Colucci WS, Liao R (2005) CD31- but Not CD31+ cardiac side population cells exhibit functional cardiomyogenic differentiation. Circ Res. 97:52-61.

90. Porrello ER, Mahmoud AI, Simpson E, Hill JA, Richardson JA, Olson EN, Sadek HA (2011) Transient regenerative potential of the neonatal mouse heart. Science. 331:1078-80.

91. Portal L, Martin V, Assaly R, d'Anglemont de Tassigny A, Michineau S, Berdeaux A, Ghaleh B, Pons S (2013) A model of hypoxia-reoxygenation on isolated adult mouse cardiomyocytes: characterization, comparison with ischemiareperfusion, and application to the cardioprotective effect of regular treadmill exercise. J Cardiovasc Pharmacol Ther. 18:367-75.

92. Raphael Rubin DSS. Rubin's Pathology. Clinicopathologic Foundations of Medicine. 2008.

93. Reubinoff BE, Pera MF, Fong CY, Trounson A, Bongso A (2000) Embryonic stem cell lines from human blastocysts: somatic differentiation in vitro. Nat Biotechnol. 18:399-404.

94. Roncalli J, Mouquet F, Piot C, Trochu JN, Le Corvoisier P, Neuder Y, Le Tourneau T, Agostini D, Gaxotte V, Sportouch C, Galinier M, Crochet D, Teiger E, Richard MJ, Polge AS, Beregi JP, Manrique A, Carrie D, Susen S, Klein B, Parini A, Lamirault G, Croisille P, Rouard H, Bourin P, Nguyen JM, Delasalle B, Vanzetto G, Van Belle E, Lemarchand P (2011) Intracoronary autologous mononucleated bone marrow cell infusion for acute myocardial infarction: results of the randomized multicenter BONAMI trial. Eur Heart J. 32:1748-57.

95. Rota M, Padin-Iruegas ME, Misao Y, De Angelis A, Maestroni S, FerreiraMartins J, Fiumana E, Rastaldo R, Arcarese ML, Mitchell TS, Boni A, Bolli R, 
Urbanek K, Hosoda T, Anversa P, Leri A, Kajstura J (2008) Local activation or implantation of cardiac progenitor cells rescues scarred infarcted myocardium improving cardiac function. Circ Res. 103:107-16.

96. Rovetto MJ, Lamberton WF, Neely JR (1975) Mechanisms of glycolytic inhibition in ischemic rat hearts. Circ Res. 37:742-51.

97. Rubio D, Garcia-Castro J, Martin MC, de la Fuente R, Cigudosa JC, Lloyd AC, Bernad A (2005) Spontaneous human adult stem cell transformation. Cancer Res. 65:3035-9.

98. Sacchetti B, Funari A, Michienzi S, Di Cesare S, Piersanti S, Saggio I, Tagliafico E, Ferrari S, Robey PG, Riminucci M, Bianco P (2007) Self-renewing osteoprogenitors in bone marrow sinusoids can organize a hematopoietic microenvironment. Cell. 131:324-36.

99. Sagoo MS, Mehta JS, Hau S, Irion LD, Curry A, Bonshek RE, Tuft SJ (2007) Microsporidium stromal keratitis: in vivo confocal findings. Cornea. 26:870-3.

100. Sainz J, Al Haj Zen A, Caligiuri G, Demerens C, Urbain D, Lemitre M, Lafont A (2006) Isolation of "side population" progenitor cells from healthy arteries of adult mice. Arterioscler Thromb Vasc Biol. 26:281-6.

101. Schofield CJ, Ratcliffe PJ (2004) Oxygen sensing by HIF hydroxylases. Nature Reviews Molecular Cell Biology. 5:343-354.

102. Senyo SE, Steinhauser ML, Pizzimenti CL, Yang VK, Cai L, Wang M, Wu TD, Guerquin-Kern JL, Lechene CP, Lee RT (2013) Mammalian heart renewal by preexisting cardiomyocytes. Nature. 493:433-6.

103. Smart N, Bollini S, Dube KN, Vieira JM, Zhou B, Davidson S, Yellon D, Riegler J, Price AN, Lythgoe MF, Pu WT, Riley PR (2011) De novo cardiomyocytes from within the activated adult heart after injury. Nature. 474:640-4.

104. Smith CL, Baek ST, Sung CY, Tallquist MD (2011) Epicardial-derived cell epithelial-to-mesenchymal transition and fate specification require PDGF receptor signaling. Circ Res. 108:e15-26. 
105. Smits $\mathrm{AM}$, van Vliet $\mathrm{P}$, Metz $\mathrm{CH}$, Korfage $\mathrm{T}$, Sluijter JP, Doevendans $\mathrm{PA}$, Goumans MJ (2009) Human cardiomyocyte progenitor cells differentiate into functional mature cardiomyocytes: an in vitro model for studying human cardiac physiology and pathophysiology. Nat Protoc. 4:232-43.

106. Soong PL, Tiburcy M, Zimmermann W-H (2012) Cardiac Differentiation of Human Embryonic Stem Cells and their Assembly into Engineered Heart Muscle. Curr Protoc Cell Biol. 55:23.8.1-23.8.21.

107. Stefanova I, Horejsi V, Ansotegui IJ, Knapp W, Stockinger H (1991) GPIanchored cell-surface molecules complexed to protein tyrosine kinases. Science. 254:1016-9.

108. Sultana N, Zhang L, Yan J, Chen J, Cai W, Razzaque S, Jeong D, Sheng W, Bu L, Xu M, Huang GY, Hajjar RJ, Zhou B, Moon A, Cai CL (2015) Resident C-kit(+) cells in the heart are not cardiac stem cells. Nat Commun. 6:8701.

109. Suzuki G (2015) Translational research of adult stem cell therapy. World $J$ Cardiol. 7:707-18.

110. Takamiya M, Haider $\mathrm{KH}$, Ashraf $\mathrm{M}$ (2011) Identification and characterization of a novel multipotent sub-population of Sca-1(+) cardiac progenitor cells for myocardial regeneration. PLoS One. 6:e25265.

111. Takehara N, Nagata M, Ogata T, Nakamura T, Matoba S, Gojo S, Sawada T, Yaku H, Matsubara H. The ALCADIA (Autologous Human Cardiac-derived Stem Cell To Treat Ischemic Cardiomyopathy) trial. 2012.

112. Tallini YN, Greene KS, Craven M, Spealman A, Breitbach M, Smith J, Fisher PJ, Steffey M, Hesse M, Doran RM, Woods A, Singh B, Yen A, Fleischmann BK, Kotlikoff MI (2009) c-kit expression identifies cardiovascular precursors in the neonatal heart. Proc Natl Acad Sci U S A. 106:1808-13.

113. Tang XL, Rokosh G, Sanganalmath SK, Yuan F, Sato H, Mu J, Dai S, Li C, Chen N, Peng Y, Dawn B, Hunt G, Leri A, Kajstura J, Tiwari S, Shirk G, Anversa P, Bolli R (2010) Intracoronary administration of cardiac progenitor cells alleviates left ventricular dysfunction in rats with a 30-day-old infarction. Circulation. 121:293-305. 
114. Tang XL, Li Q, Rokosh G, Sanganalmath SK, Chen N, Ou Q, Stowers H, Hunt G, Bolli R (2016) Long-Term Outcome of Administration of c-kitPOS Cardiac Progenitor Cells After Acute Myocardial Infarction: Transplanted Cells Do not Become Cardiomyocytes, but Structural and Functional Improvement and Proliferation of Endogenous Cells Persist for at Least One Year. Circ Res. 118:1091105.

115. Taniguchi N, Nakamura T, Sawada T, Matsubara K, Furukawa K, Hadase M, Nakahara Y, Matsubara H (2010) Erythropoietin prevention trial of coronary restenosis and cardiac remodeling after ST-elevated acute myocardial infarction (EPOC-AMI): a pilot, randomized, placebo-controlled study. Circ J. 74:2365-71.

116. Tateishi K, Ashihara E, Takehara N, Nomura T, Honsho S, Nakagami T, Morikawa S, Takahashi T, Ueyama T, Matsubara H, Oh H (2007) Clonally amplified cardiac stem cells are regulated by Sca-1 signaling for efficient cardiovascular regeneration. J Cell Sci. 120:1791-800.

117. Terrovitis JV, Smith RR, Marban E (2010) Assessment and optimization of cell engraftment after transplantation into the heart. Circ Res. 106:479-94.

118. Tiburcy M, Didie M, Boy O, Christalla P, Doker S, Naito H, Karikkineth BC, ElArmouche A, Grimm M, Nose M, Eschenhagen T, Zieseniss A, Katschinski DM, Hamdani N, Linke WA, Yin X, Mayr M, Zimmermann WH (2011) Terminal Differentiation, Advanced Organotypic Maturation, and Modeling of Hypertrophic Growth in Engineered Heart Tissue. Circulation Research. 109:1105-1114.

119. Tiburcy M, Meyer T, Soong PL, Zimmermann WH (2014) Collagen-based engineered heart muscle. Methods Mol Biol. 1181:167-76.

120. Tillmanns J, Rota M, Hosoda T, Misao Y, Esposito G, Gonzalez A, Vitale S, Parolin C, Yasuzawa-Amano S, Muraski J, De Angelis A, Lecapitaine N, Siggins RW, Loredo M, Bearzi C, Bolli R, Urbanek K, Leri A, Kajstura J, Anversa P (2008) Formation of large coronary arteries by cardiac progenitor cells. Proc Natl Acad Sci U S A. 105:1668-73.

121. Tohyama S, Hattori F, Sano M, Hishiki T, Nagahata $Y$, Matsuura $T$, Hashimoto H, Suzuki T, Yamashita H, Satoh Y, Egashira T, Seki T, Muraoka N, Yamakawa H, 
Ohgino Y, Tanaka T, Yoichi M, Yuasa S, Murata M, Suematsu M, Fukuda K (2013) Distinct metabolic flow enables large-scale purification of mouse and human pluripotent stem cell-derived cardiomyocytes. Cell Stem Cell. 12:127-37.

122. Tomita $\mathrm{Y}$, Matsumura $\mathrm{K}$, Wakamatsu $\mathrm{Y}$, Matsuzaki $\mathrm{Y}$, Shibuya I, Kawaguchi $\mathrm{H}$, leda M, Kanakubo S, Shimazaki T, Ogawa S, Osumi N, Okano H, Fukuda K (2005) Cardiac neural crest cells contribute to the dormant multipotent stem cell in the mammalian heart. J Cell Biol. 170:1135-46.

123. Torella D, Indolfi C, Goldspink DF, Ellison GM (2008) Cardiac stem cell-based myocardial regeneration: towards a translational approach. Cardiovasc Hematol Agents Med Chem. 6:53-9.

124. Tossios $P$, Krausgrill $B$, Schmidt $M$, Fischer $T$, Halbach $M$, Fries JW, Fahnenstich S, Frommolt P, Heppelmann I, Schmidt A, Schomacker K, Fischer JH, Bloch W, Mehlhorn U, Schwinger RH, Muller-Ehmsen J (2008) Role of balloon occlusion for mononuclear bone marrow cell deposition after intracoronary injection in pigs with reperfused myocardial infarction. Eur Heart J. 29:1911-21.

125. Trapnell C, Roberts A, Goff L, Pertea G, Kim D, Kelley DR, Pimentel H, Salzberg SL, Rinn JL, Pachter $L$ (2012) Differential gene and transcript expression analysis of RNA-seq experiments with TopHat and Cufflinks. Nat Protoc. 7:562-78.

126. Uchida S, De Gaspari P, Kostin S, Jenniches K, Kilic A, Izumiya Y, Shiojima I, Grosse Kreymborg K, Renz H, Walsh K, Braun T (2013) Sca1-derived cells are a source of myocardial renewal in the murine adult heart. Stem Cell Reports. 1:397410.

127. Urbanek K, Quaini F, Tasca G, Torella D, Castaldo C, Nadal-Ginard B, Leri A, Kajstura J, Quaini E, Anversa P (2003) Intense myocyte formation from cardiac stem cells in human cardiac hypertrophy. Proc Natl Acad Sci U S A. 100:10440-5.

128. Valente M, Nascimento DS, Cumano A, Pinto-do OP (2014) Sca-1+ cardiac progenitor cells and heart-making: a critical synopsis. Stem Cells Dev. 23:2263-73. 
129. van Berlo $\mathrm{JH}$, Kanisicak $\mathrm{O}$, Maillet $\mathrm{M}$, Vagnozzi RJ, Karch J, Lin S-CJ, Middleton RC, Marbán E, Molkentin JD (2014) c-kit+ cells minimally contribute cardiomyocytes to the heart. Nature. 509:337-341.

130. van de Rijn M, Heimfeld S, Spangrude GJ, Weissman IL (1989) Mouse hematopoietic stem-cell antigen Sca-1 is a member of the Ly- 6 antigen family. Proc Natl Acad Sci U S A. 86:4634-8.

131. van der Spoel TI, Jansen of Lorkeers SJ, Agostoni P, van Belle E, Gyongyosi M, Sluijter JP, Cramer MJ, Doevendans PA, Chamuleau SA (2011) Human relevance of pre-clinical studies in stem cell therapy: systematic review and meta-analysis of large animal models of ischaemic heart disease. Cardiovasc Res. 91:649-58.

132. Viola RJ, Provenzale JM, Li F, Li CY, Yuan H, Tashjian J, Dewhirst MW (2008) In vivo bioluminescence imaging monitoring of hypoxia-inducible factor 1alpha, a promoter that protects cells, in response to chemotherapy. AJR Am J Roentgenol. 191:1779-84.

133. Volz KS, Jacobs AH, Chen HI, Poduri A, McKay AS, Riordan DP, Kofler N, Kitajewski J, Weissman I, Red-Horse K (2015) Pericytes are progenitors for coronary artery smooth muscle. Elife. 4.

134. Wang X, Hu Q, Nakamura Y, Lee J, Zhang G, From AH, Zhang J (2006) The role of the sca-1+/CD31- cardiac progenitor cell population in postinfarction left ventricular remodeling. Stem Cells. 24:1779-88.

135. Warejcka DJ, Harvey R, Taylor BJ, Young HE, Lucas PA (1996) A population of cells isolated from rat heart capable of differentiating into several mesodermal phenotypes. J Surg Res. 62:233-42.

136. Wilson A, Oser GM, Jaworski M, Blanco-Bose WE, Laurenti E, Adolphe C, Essers MA, Macdonald HR, Trumpp A (2007) Dormant and self-renewing hematopoietic stem cells and their niches. Ann N Y Acad Sci. 1106:64-75.

137. Wong SP, Rowley JE, Redpath AN, Tilman JD, Fellous TG, Johnson JR (2015) Pericytes, mesenchymal stem cells and their contributions to tissue repair. Pharmacol Ther. 151:107-20. 
138. Yancy CW, Jessup M, Bozkurt B, Butler J, Casey DE, Jr., Drazner MH, Fonarow GC, Geraci SA, Horwich T, Januzzi JL, Johnson MR, Kasper EK, Levy WC, Masoudi FA, McBride PE, McMurray JJ, Mitchell JE, Peterson PN, Riegel B, Sam F, Stevenson LW, Tang WH, Tsai EJ, Wilkoff BL (2013) 2013 ACCF/AHA guideline for the management of heart failure: a report of the American College of Cardiology Foundation/American Heart Association Task Force on practice guidelines. Circulation. 128:e240-327.

139. Ye J, Boyle A, Shih H, Sievers RE, Zhang Y, Prasad M, Su H, Zhou Y, Grossman W, Bernstein HS, Yeghiazarians Y (2012) Sca-1+ cardiosphere-derived cells are enriched for Isl1-expressing cardiac precursors and improve cardiac function after myocardial injury. PLoS One. 7:e30329.

140. Zangi L, Lui KO, von Gise A, Ma Q, Ebina W, Ptaszek LM, Spater D, Xu H, Tabebordbar M, Gorbatov R, Sena B, Nahrendorf M, Briscoe DM, Li RA, Wagers AJ, Rossi DJ, Pu WT, Chien KR (2013) Modified mRNA directs the fate of heart progenitor cells and induces vascular regeneration after myocardial infarction. Nat Biotechnol. 31:898-907.

141. Zaruba MM, Soonpaa M, Reuter S, Field LJ (2010) Cardiomyogenic Potential of C-Kit+-Expressing Cells Derived From Neonatal and Adult Mouse Hearts. Circulation. 121:1992-2000.

142. Zimmermann WH, Fink C, Kralisch D, Remmers U, Weil J, Eschenhagen $T$ (2000) Three-dimensional engineered heart tissue from neonatal rat cardiac myocytes. Biotechnol Bioeng. 68:106-14.

143. Zimmermann $\mathrm{WH}$, Schneiderbanger $\mathrm{K}$, Schubert $\mathrm{P}$, Didie M, Munzel $\mathrm{F}$, Heubach JF, Kostin S, Neuhuber WL, Eschenhagen T (2002) Tissue engineering of a differentiated cardiac muscle construct. Circ Res. 90:223-30. 


\section{Appendix}

\section{A1. Reagents and medium}

\section{Reagents and medium for cell culture:}

\section{$\underline{\text { GF-1 } 1 \text { stock solution }}$}

Reconstitute IGF-1 (Recombinant Human IGF-1, \#AF-100-11, Peprotech) in sterile 1x PBS with $0.1 \%$ human recombinant serum albumin (HSA) (\#A9731, SigmaAldrich) according to manufacturer's protocol to obtain a stock concentration of 100 $\mu \mathrm{g} / \mathrm{ml}$; aliquots were stored at $-20^{\circ} \mathrm{C}$ until further use.

\section{$\underline{\text { bFGF stock solution (Peprotech) }}$}

Reconstitute bFGF (Recombinant Human FGF-basic [154 a.a], \#AF-100-18B, Peprotech) in sterile 1x PBS with 0.1\% HSA (\#A9731, Sigma-Aldrich) according to manufacturer's protocol to obtain a stock concentration of $10 \mu \mathrm{g} / \mathrm{m}$; aliquots were stored at $-20{ }^{\circ} \mathrm{C}$ until further use.

\section{$\underline{\text { bFGF stock solution (Miltenyi Biotech) }}$}

Reconstitute bFGF (Human FGF-2 premium grade, \#130-093-841, Miltenyi Biotech) in sterile $1 \mathrm{x}$ PBS with $0.1 \%$ HSA (\#A9731, Sigma-Aldrich) according to manufacturer's protocol to obtain a stock concentration of $10 \mu \mathrm{g} / \mathrm{ml}$; aliquots were stored at $-20{ }^{\circ} \mathrm{C}$ until further use.

\section{VEGF-A stock solution}

Reconstitute VEGF-A (Animal-Free Recombinant Human VEGF [165], \#AF-100-20, Peprotech) in sterile 1x PBS with 0.1\% HSA (\#A9731, Sigma-Aldrich) according to manufacturer's protocol to obtain a stock concentration of $5 \mu \mathrm{g} / \mathrm{ml}$; aliquots were stored at $-20{ }^{\circ} \mathrm{C}$ until further use. 


\section{$\underline{\text { TGF- } \beta 1 \text { stock solution }}$}

Reconstitute TGF- $\beta 1$ (Recombinant Human TGF- $\beta 1$ [CHO cell derived], \#AF-10021C, Peprotech) in sterile 1x PBS with 0.1\% HAS (\#A9731, Sigma-Aldrich) according to manufacturer's protocol to obtain a stock concentration of $5 \mu \mathrm{g} / \mathrm{ml}$; aliquots were stored at $-20^{\circ} \mathrm{C}$ until further use.

\section{EGF stock solution}

Reconstitute EGF (Animal-Free Recombinant Human EGF, \#AF-100-18B, Peprotech) in sterile 1x PBS with 0.1\% HSA (\#A9731, Sigma-Aldrich) according to manufacturer's protocol to obtain a stock concentration of $10 \mu \mathrm{g} / \mathrm{ml}$; aliquots were stored at $-20{ }^{\circ} \mathrm{C}$ until further use.

\section{BMP-4 stock solution}

Reconstitute BMP-4 (Recombinant Human BMP-4, \#314-BP, R\&D Systems) in sterile 1x PBS with 0.1\% HSA (\#A9731, Sigma-Aldrich) according to manufacturer's protocol to obtain a stock concentration of $10 \mu \mathrm{g} / \mathrm{ml}$; aliquots were stored at $-20{ }^{\circ} \mathrm{C}$ until further use.

\section{Activin A stock solution}

Reconstitute Activin A (Recombinant Human/Mouse/Rat Activin A Protein, \#338-AC, R\&D Systems) in sterie 1x PBS with 0.1\% HSA (\#A9731, Sigma-Aldrich) according to manufacturer's protocol to obtain a stock concentration of $10 \mu \mathrm{g} / \mathrm{ml}$; aliquots were stored at $-20{ }^{\circ} \mathrm{C}$ until further use.

\section{$\underline{\text { CHIR stock solution }}$}

Reconstitute CHIR (Stemolecule ${ }^{T M}$ CHIR99021, \#04-0004, Stemgent) in DMSO according to manufacturer's protocol to obtain a stock concentration of $10 \mathrm{mmol} / \mathrm{L}$; aliquots were stored at $-20^{\circ} \mathrm{C}$ until further use. 


\section{IWP-4 stock solution}

Reconstitute IWP-4 (Stemolecule ${ }^{\mathrm{TM}}$ Wnt Inhibitor IWP-4, \#04-0036, Stemgent) in DMSO according to manufacturer's protocol to obtain a stock concentration of 5 $\mathrm{mmol} / \mathrm{L}$; aliquots were stored at $-20^{\circ} \mathrm{C}$ until further use.

\section{$\underline{\text { Nucleoside Mix (100x) }}$}

Dissolve $80 \mathrm{mg}$ Adenosine (final con. $30 \mu \mathrm{mol} / \mathrm{L}$, \#A4036, Sigma), $85 \mathrm{mg}$ Guanosine (final con. $30 \mu \mathrm{mol} / \mathrm{L}$, \#G6264, Sigma), $73 \mathrm{mg}$ Cytidine (final con. $30 \mu \mathrm{mol} / \mathrm{L}$, \#4654, Sigma), $73 \mathrm{mg}$ Uridine (final con. $30 \mu \mathrm{mol} / \mathrm{L}$, \#U-3003, Sigma) and $24 \mathrm{mg}$ Thymidine (final con. $10 \mu \mathrm{mol} / \mathrm{L}, \# \mathrm{~T} 1895$, Sigma) in 1x PBS, sterile filter; aliquots were stored at $-20{ }^{\circ} \mathrm{C}$ until further use.

\section{Ascorbic acid stock solution}

Dissolve $0.87 \mathrm{~g} \mathrm{L-ascorbic} \mathrm{acid} \mathrm{2-phosphate} \mathrm{sesquimagnesium} \mathrm{salt} \mathrm{hydrate} \mathrm{( \# A8960,}$ Sigma-Aldrich) in $10 \mathrm{ml}$ of $\mathrm{dd}_{2} \mathrm{O}$ to obtain $300 \mathrm{mmol} / \mathrm{L}$ stock concentration, sterile filter; aliquots were stored at $-20^{\circ} \mathrm{C}$ until further use.

\section{CMR supplement (50x)}

$\begin{array}{ll}\text { Albumin Human (\#A9731, Sigma-Aldrich) } & 1.25 \mathrm{~g} \\ \text { Triiodothyronine (T3), } & 10 \mu \mathrm{gg} \\ \text { Transferrin } & 25 \mathrm{mg} \\ \text { Sodium Selenite } & 80 \mu \mathrm{gg} \\ \text { > adjust with Aq. dest. water up to } 50 \mathrm{~mL} & \\ \text { > keep aliquots at }-20^{\circ} \mathrm{C} . & \end{array}$

\section{0x DMEM}

Dissolve 1.34 g DMEM powder (DMEM, powder, high glucose, \#52100-039, Gibco) in $10 \mathrm{ml}$ of $\mathrm{ddH}_{2} \mathrm{O}$, sterile filter and keep at $4{ }^{\circ} \mathrm{C}$.

\section{0x RPMI}

Dissolve $1.04 \mathrm{~g}$ RPMI powder (RPMI 1640 medium, powder, \#52800-035, Gibco) in $10 \mathrm{ml}$ of $\mathrm{ddH}_{2} \mathrm{O}$, sterile filter and keep at $4{ }^{\circ} \mathrm{C}$. 


\section{$\underline{\text { Rat EHM medium }}$}

Iscove's medium (\#F0465, Biochrom) $\quad 500 \mathrm{ml}$

Horse serum (\#16050, Gibco) $58 \mathrm{ml}$

Chich embryo extract $\quad 11.6 \mathrm{ml}$

Penicilin (Pen)/Streptomycin (Strep) (100x, Pen:

10,000 U/mL and Strep: 10,000 $\mu \mathrm{g} / \mathrm{mL}$, \#15140- $5.8 \mathrm{ml}$

122, Gibco)

$\underline{\text { mESC medium }}$

DMEM, high glucose, HEPES (\#42430-025, Gibco) $500 \mathrm{ml}$

Fetal Bovine Serum (FBS) (\#10270, Gibco) $92 \mathrm{ml}$

L-Glutamine (200 mmol/L, \#25030-024, Gibco) $\quad 6.2 \mathrm{ml}$

Pen/Strep (100x, \#15140-122, Gibco) $6.2 \mathrm{ml}$

MEM Non-Essential Amino Acids Solution (NEAA) $6.2 \mathrm{ml}$ (100x, \#111450-035, Gibco)

$\begin{array}{ll}\text { Sodium Pyruvate (100 mmol/L, \#11360-039, Gibco) } & 6.2 \mathrm{ml} \\ \text { Nucleoside mix (100x) } & 6.2 \mathrm{ml}\end{array}$

Leukemia Inhibitory factor (LIF) (1000 Units/ml, $\quad 62 \mu \mathrm{l}$

\#ESG1106, Esgro)

2- $\beta$-mercaptoethanol (31350-010, Sigma) $4.34 \mu \mathrm{l}$

mESC differentiation (diff.) medium / mEHM

$\underline{\text { medium }}$

Iscove's medium (\#F0465, Biochrom) $\quad 500 \mathrm{ml}$

FBS (\#10270, Gibco) $120 \mathrm{ml}$

L-Glutamine (200 mmol/L, \#25030-024, Gibco) $\quad 6.2 \mathrm{ml}$

Pen/Strep (100x, \#15140-122, Gibco) $6.2 \mathrm{ml}$

MEM-NEAA (100x, \#111450-035, Gibco) $6.2 \mathrm{ml}$

L-Ascorbic acid 2-phosphate sesquimagnesium salt hydrate (Ascorbic acid for cell culture, \#A8960,

Sigma)

2- $\beta$-mercaptoethanol (31350-010, Sigma) $\quad 4.34 \mu \mathrm{l}$ 
$\underline{\mathrm{mCPC} \text { medium (incomplete) }}$

DMEM/F-12 medium (\#31300-038, Gibco) $500 \mathrm{ml}$

FBS (\#10270, Gibco) $50 \mathrm{ml}$

Pen/Strep (100x, \#15140-122, Gibco) $5.5 \mathrm{ml}$

L-Glutamine (200 mmol/L, \#25030-024, Gibco) $5.5 \mathrm{ml}$

Insulin-Transferrin-Selenium-Ethanolamine (ITS-X) $5.5 \mathrm{ml}$

(100x, \# 51500-056, Gibco)

$50 \mu \mathrm{l}$

$\underline{\text { mCPC medium }}$

mCPC medium (incomplete) $\quad 50 \mathrm{ml}$

EGF stock solution

bFGF stock solution

$200 \mu \mathrm{l}$ (final con: $40 \mathrm{ng} / \mathrm{mL}$ )

$100 \mu \mathrm{l}$ (final con: $20 \mathrm{ng} / \mathrm{mL}$ )

$\underline{\text { mEF medium }}$

DMEM medium ( \#61965-026, Gibco) $500 \mathrm{ml}$

FBS (\#10270, Gibco) $50 \mathrm{ml}$

Pen/Strep (100x, \#15140-122, Gibco) $5.5 \mathrm{ml}$

MEM-NEAA (100x, \#111450-035, Gibco) $5.5 \mathrm{ml}$

$\underline{\text { hCM medium }}$ RPMI medium 1640, GlutaMAX ${ }^{\text {TM }}$ (\#61870-010, $500 \mathrm{ml}$
Gibco)

$5.2 \mathrm{ml}$

Pen/Strep (100x, \#15140-122, Gibco)

$\begin{array}{ll}\text { Sodium Pyruvate (100 mM, \#11360-039, Gibco) } & 5.2 \mathrm{ml} \\ \text { Ascorbic acid stock solution (300 mmol/L) } & 333 \mu \mathrm{l}\end{array}$

B-27® Supplement (50x, \#17504-044, Gibco) 10 ml

$\underline{\mathrm{hEHM} \text { medium (incomplete) }}$

Iscove's medium (\#F0465, Biochrom) $500 \mathrm{ml}$

L-Glutamine (200 mmol/L, \#25030-024, Gibco) $\quad 5.5 \mathrm{ml}$

Pen/Strep (100x, \#15140-122, Gibco) $5.5 \mathrm{ml}$

MEM-NEAA (100x, \#111450-035, Gibco) 5.5. ml

Ascorbic acid stock solution ( $300 \mathrm{mmol} / \mathrm{L}) \quad 500 \mu \mathrm{l}$ 


\section{$\underline{\mathrm{hEHM} \text { medium }}$}

hEHM medium (incomplete)

$50 \mathrm{ml}$

B-27® Supplement, minus insulin (50x, \#A18956-

01, Gibco)

$2 \mathrm{ml}$

IGF-1 stock solution

$50 \mu \mathrm{l}$ (final con: $100 \mathrm{ng} / \mathrm{mL}$ )

bFGF stock solution

$50 \mu \mathrm{l}$ (final con: $10 \mathrm{ng} / \mathrm{mL}$ )

VEGF-A stock solution

$50 \mu \mathrm{l}$ (final con: $5 \mathrm{ng} / \mathrm{mL}$ )

$\underline{\text { hEHM consolidation medium }}$

hEHM medium

$50 \mathrm{ml}$

TGF- $\beta$ stock solution

$50 \mu l$ (final con: $5 \mathrm{ng} / \mathrm{mL}$ )

$\underline{\text { hFF medium }}$

DMEM, high glucose, HEPES (\#42430-025, Gibco) $500 \mathrm{ml}$

FBS (\#10270, Gibco)

$75 \mathrm{ml}$

Pen/Strep (100x, \#15140-122, Gibco)

$5.8 \mathrm{ml}$

DMEM SF-B27 medium

DMEM, low glucose, GlutaMAX TM, Pyruvate

(\#21885-025, Gibco)

Pen/Strep (100x, \#15140-122, Gibco)

MEM-NEAA (100x, \#111450-035, Gibco)

B-27@ Supplement, minus insulin (50x, \#A18956-

01, Gibco)

$500 \mathrm{ml}$

$5 \mathrm{ml}$

$5 \mathrm{ml}$

$20 \mathrm{ml}$

\section{DMEM SF-CMR medium}

DMEM, low glucose, GlutaMAX тM, Pyruvate

(\#21885-025, Gibco)

$500 \mathrm{ml}$

Pen/Strep (100x, \#15140-122, Gibco)

$5 \mathrm{ml}$

MEM-NEAA (100x, \#111450-035, Gibco)

$5 \mathrm{ml}$

CMR supplement (50x)

$10 \mathrm{ml}$ 


\section{2x Rat EHM DMEM}

10x DMEM

$1 \mathrm{ml}$

Horse serum ((\#16050, Gibco) $1 \mathrm{ml}$

Chick embryo extract

$0.2 \mathrm{ml}$

Pen/Strep (100x, \#15140-122, Gibco)

$0.1 \mathrm{ml}$

$\mathrm{ddH}_{2} \mathrm{O}$

$2.7 \mathrm{ml}$

\section{2x Mouse EHM DMEM}

10x DMEM

FBS (\#10270, Gibco)

Pen/Strep (100x, \#15140-122, Gibco)

$\mathrm{ddH}_{2} \mathrm{O}$

\section{$\underline{\text { 2x Human EHM RPMI }}$}

10x RPMI

B-27® Supplement, minus insulin (50x, \#A18956-

01, Gibco)

Pen/Strep (100x, \#15140-122, Gibco)

$\mathrm{ddH}_{2} \mathrm{O}$

$\underline{\text { hESC medium }}$

KO DMEM medium (\#10829, Invitrogen)

KSR (Knockout Serum Replacement) (\#10828, Invitrogen)

MEM-NEAA (100x, \#111450-035, Gibco)

L-Glutamine (200 mmol/L, \#25030-024, Gibco)

Pen/Strep (100x, \#15140-122, Gibco)

bFGF stock solution (Miltenyi Biotech)

$\underline{\text { hFF conditioned medium }}$

hESC medium

bFGF stock solution (Miltenyi Biotech)

$>$ add onto hFFs for 48 hours

\section{$\underline{\mathrm{hESC} \text { conditioned medium }}$}

hESC medium

$25 \mathrm{ml}$

hFF conditioned medium

bFGF stock solution (Miltenyi Biotech)
$1 \mathrm{ml}$

$2 \mathrm{ml}$

$0.2 \mathrm{ml}$

$1.8 \mathrm{ml}$

$2 \mathrm{ml}$

$0.8 \mathrm{ml}$

$0.2 \mathrm{ml}$

$7 \mathrm{ml}$

$39.5 \mathrm{ml}$

$10 \mathrm{ml}$

$0.5 \mathrm{ml}$

$0.5 \mathrm{ml}$

$0.5 \mathrm{ml}$

$50 \mu \mathrm{l}$ (final con: $10 \mathrm{ng} / \mathrm{mL}$ )

$50 \mathrm{ml}$

$25 \mu \mathrm{l}$ (final con: $5 \mathrm{ng} / \mathrm{mL}$ ) 


\section{Mesoderm induction medium}

hCM medium

CHIR stock solution

BMP4 stock solution

Activin-A stock solution

bFGF stock solution (Miltenyi Biotech)

$\underline{\text { Cardiac specification medium }}$

hCM medium

IWP4 stock solution

$\underline{\text { hCM selection medium }}$
$50 \mathrm{ml}$

$5 \mu \mathrm{l}$ (final con: $1 \mu \mathrm{mol} / \mathrm{mL}$ )

$25 \mu \mathrm{l}$ (final con: $5 \mathrm{ng} / \mathrm{mL}$ )

$45 \mu \mathrm{l}$ (final con: $9 \mathrm{ng} / \mathrm{mL}$ )

$25 \mu \mathrm{l}$ (final con: $5 \mathrm{ng} / \mathrm{mL}$ )

$50 \mathrm{ml}$

$50 \mu \mathrm{l}$ (final con: $5 \mu \mathrm{mol} / \mathrm{mL}$ )

RPMI medium 1640, without D-Glucose, without LGlutamine (\#01-101-1A, Biological Industries)

$49.15 \mathrm{ml}$

Pen/Strep (100x, \#15140-122, Gibco)

$0.5 \mathrm{ml}$

Sodium DL-Lactate solution 50\% in H2O (\#71723,

Sigma-Aldrich)

$0.25 \mathrm{ml}$

2-mercaptoethanol (50 mmol/L; 31350010;

Invitrogen)

$0.1 \mathrm{ml}$

hCPC-Conditioned medium (ConM)

hCPCs were plated and cultured in T-25 culture flask in hFF-medium with the seeding density of $1 \times 10^{6}$ cells/flask. hFF medium was replaced with DMEM SFCMR medium when $\mathrm{hCPC}$ reached $90-100 \%$ confluency. DMEM SF-CMR medium from hCPCs was collected every second day for 7 days and immediately frozen and stored at $-80^{\circ} \mathrm{C}$. hCPC conditioned medium (ConM) was prepared freshly, diluted 1:1 with fresh DMEM SF-CMR medium prior to use.

\section{$\underline{\text { hFF-Conditioned medium (ConM) }}$}

hFFs were plated and cultured in T-25 culture flask in hFF-medium with the seeding density of $1 \times 10^{6}$ cells/flask. hFF medium was replaced with DMEM SF-CMR medium when hFFs reached $90-100 \%$ confluency. DMEM SF-CMR medium from hFFs was collected every second day for 7 days and immediately frozen and stored 
at $-80{ }^{\circ} \mathrm{C}$. hFF-ConM was prepared freshly, diluted 1:1 with fresh DMEM SF-CMR medium prior to use.

\section{Collagenase digestion solution}

Dissolve $500 \mathrm{mg}$ collagenase (Collagenase from Clostridium histolyticum for general use, Type I, \# C0130, Sigma-Aldrich) in $250 \mathrm{ml}$ of $1 \times$ PBS containing $\mathrm{Ca}^{+2}$ and $\mathrm{Mg}^{+2}$ with $20 \%$ FBS (\#10270, Gibco), sterile filter and keep aliquots at $-20^{\circ} \mathrm{C}$.

\section{Dnase stock solution}

Dissolve Dnase I (DNase I, Bovine Pancreas, \#260913, Calbiochem) in $\mathrm{ddH}_{2} \mathrm{O}$ to obtain a stock concentration of $1 \mathrm{mg} / \mathrm{mL}$, keep aliquots at $-20^{\circ} \mathrm{C}$.

\section{EDTA solution}

Add $500 \mu \mathrm{l}$ of $0.5 \mathrm{M}$ EDTA solution (EDTA solution $\mathrm{pH} 8.0(0.5 \mathrm{M})$ for molecular biology (\#A4892, AppliChem) to $500 \mathrm{ml}$ of $1 \times$ PBS containing $0.45 \mathrm{~g} \mathrm{NaCl}$. Sterile filter, keep aliquots at $4{ }^{\circ} \mathrm{C}$.

\section{EB digestion protocol}

Collagenase digestion solution

$10 \mathrm{ml}$

Dnase stock solution $200 \mu \mathrm{l}$

\section{$\underline{\mathrm{hCM} \text { digestion solution }}$}

Accutase cell detachment solution, (\#SCR005, Milipore)

Trypsin (2.5\%) (\#15090-046, Gibco)

Dnase stock solution

$97.75 \mathrm{ml}$

$0.25 \mathrm{ml}$

$2 \mathrm{ml}$ 
Reagents for isometric force measurement:

$\underline{\mathrm{CaCl}}_{2}$ stock solution $(2.25 \mathrm{~mol} / \mathrm{L})$

$\mathrm{CaCl}_{2} \times 2 \mathrm{H}_{2} \mathrm{O}$ (Mw: $\left.147.02 \mathrm{~g} / \mathrm{mol}\right) \quad 165.57 \mathrm{~g}$

$\mathrm{ddH}_{2} \mathrm{O}$

$500 \mathrm{ml}$

$\underline{\mathrm{MgCl} 2 \text { stock solution }(1.05 \mathrm{~mol} / \mathrm{L})}$

$\mathrm{MgCl}_{2} \times 6 \mathrm{H}_{2} \mathrm{O}(\mathrm{Mw}: 203.01 \mathrm{~g} / \mathrm{mol}) \quad 106.83 \mathrm{~g}$

$\mathrm{ddH}_{2} \mathrm{O}$

$500 \mathrm{ml}$

Stock I solution ( $0.2 \mathrm{mmol} / \mathrm{L}$ Calcium)

$\mathrm{NaCl}$

$175 \mathrm{~g}$

$\mathrm{KCl}$

$10 \mathrm{~g}$

$\mathrm{CaCl}_{2}$ stock solution $(2.25 \mathrm{~mol} / \mathrm{L}) \quad 2.22 \mathrm{ml}$

$\mathrm{MgCl}_{2}$ stock solution $(1.05 \mathrm{~mol} / \mathrm{L}) \quad 25 \mathrm{ml}$

$\mathrm{ddH}_{2} \mathrm{O}$ up to $1 \mathrm{~L}$

$\underline{\text { Stock II solution }}$

$\mathrm{NaHCO}_{3}$

$50 \mathrm{~g}$

$\mathrm{ddH}_{2} \mathrm{O}$

Stock III solution

$\mathrm{NaH}_{2} \mathrm{PO}_{4}$

$5.8 \mathrm{~g}$

$\mathrm{ddH}_{2} \mathrm{O}$

$>$ keep all the stock solutions at $4{ }^{\circ} \mathrm{C}$.

Tyrode solution

Stock I solution $(0.2 \mathrm{mmol} / \mathrm{L}$ Calcium $) 200 \mathrm{ml}$

Stock II solution $190 \mathrm{ml}$

Stock III solution $50 \mathrm{ml}$

D-Glucose

$5 \mathrm{~g}$

Ascorbic acid $500 \mathrm{mg}$

$\mathrm{ddH}_{2} \mathrm{O}$ up to $5 \mathrm{~L}$

>Prepare the Tyrode solution prior to contraction force measurement. 


\section{Reagents for western blot:}

$\underline{\text { Solutions for cell lysis: }}$

Protease inhibitor stock solution (10x)

Dissolve 1 tablet of protease inhibitor (Protease inhibitor, cOmplete ULTRA Tablets, Mini, EASYpack, \#05892970001, Roche) in $1.5 \mathrm{ml}$ of $\mathrm{ddH}_{2} \mathrm{O}$, keep in aliquots at -20 C.

\section{Phosphatase inhibitor stock solution (10x)}

Dissolve 1 tablet of phosphatase inhibitor (Phosphatase inhibitor, PhosSTOP EASYpack, \#04906837001, Roche) in $1 \mathrm{ml}$ of $\mathrm{dd}_{2} \mathrm{O}$, keep aliquots at $-20^{\circ} \mathrm{C}$.

Cell lysis buffer (incomplete)

Tris- $\mathrm{HCl}(\mathrm{pH} 8.0)$

$\mathrm{NaCl}$

EDTA

$>$ keep at $4{ }^{\circ} \mathrm{C}$.

Cell lysis buffer

Cell lysis buffer (incomplete)

Protease inhibitor stock solution (10x)

Phosphatase inhibitor stock solution (10x)
$10 \mathrm{mmol} / \mathrm{L}$ $400 \mathrm{mmol} / \mathrm{L}$ $1 \mathrm{mmol} / \mathrm{L}$

Solutions for SDS-gel electrophoresis:

\section{$\underline{0.5 \mathrm{~mol} / \mathrm{L} \text { Tris- } \mathrm{HCl} \mathrm{pH} 6.8}$}

Tris- $\mathrm{HCl}$

$\mathrm{ddH}_{2} \mathrm{O}$

> adjust to $\mathrm{pH} 6.8$.

\section{$1.5 \mathrm{~mol} / \mathrm{L}$ Tris- $\mathrm{HCl} \mathrm{pH} 8.8$}

Tris- $\mathrm{HCl}$

$\mathrm{ddH}_{2} \mathrm{O}$

> adjust to $\mathrm{pH} 8.8$.
$750 \mu \mathrm{l}$

$150 \mu \mathrm{l}$

$100 \mu \mathrm{l}$ 
$\underline{\text { Laemmli loading buffer (6x) }}$

$0.5 \mathrm{~mol} / \mathrm{L}$ Tris-HCl pH $6.8 \quad 3 \mathrm{ml}$

Bromphenol blue 0.5\% $\quad 0.75 \mathrm{ml}$

SDS

$1.2 \mathrm{~g}$

Glycerin $100 \%$

$1.5 \mathrm{ml}$

2- $\beta$-Mercaptoethanol

$150 \mu \mathrm{l}$

$\mathrm{ddH}_{2} \mathrm{O}$

up to $10 \mathrm{ml}$

$>$ keep aliquots at $-20^{\circ} \mathrm{C}$.

Seperating gel $(12 \%, 2$ gels $)$

$\mathrm{dd}_{2} \mathrm{O}$

Acrylamide $30 \%$

$1.5 \mathrm{~mol} / \mathrm{L}$ Tris- $\mathrm{HCl} \mathrm{pH} 8.8$

SDS $10 \%$

APS $10 \%$

TEMED

Stacking gel (2 gels)

$\mathrm{ddH}_{2} \mathrm{O}$

Acrylamide $30 \%$

$0.5 \mathrm{~mol} / \mathrm{L}$ Tris- $\mathrm{HCl} \mathrm{pH} 6.8$

SDS $10 \%$

APS $10 \%$

TEMED

$\underline{\text { Running buffer (10X, pH 8.3-8.7) }}$

Tris-Base

Glycine

$\mathrm{ddH}_{2} \mathrm{O}$

Solutions for immunoblotting:

$\underline{\text { Transfer buffer (10x) }}$

Tris-Base

Glycine

$\mathrm{ddH}_{2} \mathrm{O}$
$60.5 \mathrm{~g}$

$2.8 \mathrm{ml}$

$0.85 \mathrm{ml}$

$1.25 \mathrm{ml}$

$50 \mu \mathrm{l}$

$50 \mu \mathrm{l}$

$5 \mu \mathrm{l}$

$30.3 \mathrm{~g}$

$144.1 \mathrm{~g}$

$1 \mathrm{~L}$

$288 \mathrm{~g}$

up to $2 \mathrm{~L}$ 
$\underline{\text { Transfer buffer }(1 \mathrm{x})}$

Transfer buffer (10x) $\quad 100 \mathrm{ml}$

Methanol $200 \mathrm{ml}$

$\mathrm{ddH}_{2} \mathrm{O} \quad$ up to $1 \mathrm{~L}$

Blocking buffer

Prepare $5 \%$ of milk powder (milk, non-fatty dried, Carl Roth) solution in 1x PBS.

\section{Reagents for Agarose gel electrophoresis:}

$\underline{\text { TAE buffer (50x) }}$

Tris-Base

$0.5 \mathrm{M}$ EDTA pH 8.0

Acetic acid

$\mathrm{ddH}_{2} \mathrm{O}$

$>$ adjust to $\mathrm{pH} 8.5$.

$\underline{\text { TAE buffer (1x) }}$

TAE buffer (50x)

$\mathrm{ddH}_{2} \mathrm{O}$

Agarose gel (1.5\%)

TAE buffer (1 $x)$

$100 \mathrm{ml}$

Agarose UltraPureTM (AppliChem) $1.5 \mathrm{~g}$
$242.2 \mathrm{~g}$

$18.6 \mathrm{~g}$

$57.1 \mathrm{~g}$

up to $1 \mathrm{~L}$

$3 \mathrm{ml}$

up to $150 \mathrm{ml}$

> Let the agarose gel cool down, pipette $0.2 \mu \mathrm{g} / \mathrm{ml}$ ethidium bromide (EtBR; Ethidium bromide solution, \#46067, Sigma-Aldrich) into the gel and directly pour into the casting tray placed with a comb inside. 
Appendix (A1. Reagents and medium)

\section{Reagents for Immunostaining:}

Permeabilizing blocking buffer for Immunocyto/histostaining and Flow cytometry

$\begin{array}{ll}\text { Goat serum } & 26.3 \mathrm{ml} \\ \text { Bovine serum albumin (BSA) (\#A3311, Sigma) } & 5.26 \mathrm{~g} \\ \text { Triton x-100 } & 2.63 \mathrm{ml} \\ \text { 1x PBS } & \text { up to } 500 \mathrm{ml}\end{array}$

Non-permeabilizing blocking buffer for Flow cytometry

Prepare 1x PBS containing 5\% FBS (\#10270, Gibco) and keep at $4{ }^{\circ} \mathrm{C}$ until use. 


\section{A2. Primers}

\begin{tabular}{|c|c|c|c|c|}
\hline Gene & & Sequence $\left(5^{\prime} \rightarrow 3^{\prime}\right)$ & $\begin{array}{l}\text { Fragment size } \\
\text { (bp) }\end{array}$ & Ensembl code \\
\hline \multirow{2}{*}{ murine Kit } & fwd & GAGCAAAGGTGTACCACTCC & \multirow{2}{*}{162} & \multirow{2}{*}{ ENSMUSG00000005672 } \\
\hline & rev & GAACTCTTGCCCACATCGTT & & \\
\hline \multirow{2}{*}{ murine $N k \times 2-5$} & fwd & CGACAGCGGCAGGACCAGAC & \multirow{2}{*}{133} & \multirow{2}{*}{ ENSMUSG00000015579 } \\
\hline & rev & CGTAGGCGGGAGCGTAGGC & & \\
\hline \multirow{2}{*}{$\begin{array}{l}\text { murine } L y 6 a(S c a- \\
\text { 1) }\end{array}$} & fwd & GCAGCAGTTATTGTGCATTCTC & \multirow{2}{*}{226} & \multirow{2}{*}{ ENSMUSG00000075602 } \\
\hline & rev & AAGGTCTGCAGGAGGACTGA & & \\
\hline \multirow{2}{*}{ murine Ddr2 } & fwd & CCGAAAGCTTCCAGAGTTTG & \multirow{2}{*}{249} & \multirow{2}{*}{ ENSMUSG00000026674 } \\
\hline & rev & TTCTCCCAGCTTCTCCTTGA & & \\
\hline \multirow{2}{*}{ murine Gapdh } & fwd & $\begin{array}{l}\text { ATGTTCCAGTATGACTCCACTCA } \\
\text { CG }\end{array}$ & \multirow{2}{*}{170} & \multirow{2}{*}{ ENSMUSG00000057666 } \\
\hline & rev & $\begin{array}{l}\text { GAAGACACCAGTAGACTCCACGA } \\
\text { CA }\end{array}$ & & \\
\hline \multirow{2}{*}{ human $K I T$} & fwd & TTGTTAGAGATCCTGCCAAGC & \multirow{2}{*}{209} & \multirow{2}{*}{ ENSG00000157404 } \\
\hline & rev & GTAGGCGCGTTTCACACTTT & & \\
\hline \multirow{2}{*}{ human PECAM1 } & fwd & GGTGGAAGGAGTGCCCAG T & \multirow{2}{*}{114} & \multirow{2}{*}{ ENSG00000261371 } \\
\hline & rev & GTGAAGTGTATTGGGGCCTTT & & \\
\hline \multirow{2}{*}{ human $A C T A 2$} & fwd & GGAAAAGATCTGGCACCACTC & \multirow{2}{*}{196} & \multirow{2}{*}{ ENSG00000107796 } \\
\hline & rev & GCGTCCAGAGGCATAGAGAG & & \\
\hline \multirow{2}{*}{ human NKX2-5 } & fwd & GACCCTAGAGCCGAAAAGAA & \multirow{2}{*}{227} & \multirow{2}{*}{ ENSG00000183072 } \\
\hline & rev & GTGGACGTGAGTTTCAGCAC & & \\
\hline \multirow{2}{*}{ human $A C T C 1$} & fwd & CCGGGAGAAGATGACTCAGA & \multirow{2}{*}{170} & \multirow{2}{*}{ ENSG00000159251 } \\
\hline & rev & GCAAAGCGTAGCCCTCATAG & & \\
\hline \multirow{2}{*}{ human TNNI3 } & fwd & CTCACTGACССТCCAAACG & \multirow{2}{*}{206} & \multirow{2}{*}{ ENSG00000129991 } \\
\hline & rev & AATTTTCTCGAGGCGGAGA & & \\
\hline & fwd & CTAAGACACCAGCAGCTCCTTC & & \\
\hline human GATA4 & rev & GTGCCCGTAGTGAGATGACAG & 146 & ENSG00000136574 \\
\hline & fwd & CGACCCTCACATCAAGCTACA & & \\
\hline human $F G F 2$ & rev & CGTTTCAGTGCCACAACATACCA & 219 & ENSG00000138685 \\
\hline human VEGFA & fwd & CACGAAGTGGTGAAGTTCATGG & 121 & ENSG00000112715 \\
\hline
\end{tabular}


Appendix (A2. Primers)

\begin{tabular}{|c|c|c|c|c|}
\hline & rev & CACAGGATGGCTTGAAGATGT & & \\
\hline \multirow{2}{*}{ human $H G F$} & fwd & CCTATGCAGAGGGACAAAGGA & \multirow{2}{*}{133} & \multirow{2}{*}{ ENSG00000019991 } \\
\hline & rev & GCACATTGGTCTGCAGTATTCA & & \\
\hline \multirow{2}{*}{ human IGF1 } & fwd & ATTTCAACAAGCCCACAGGGTA & \multirow{2}{*}{511} & \multirow{2}{*}{ ENSG00000017427 } \\
\hline & rev & AGGGGTGCGCAATACATCT & & \\
\hline \multirow{2}{*}{ human $P D G F$} & fwd & GAGAAGCATCGAGGAAGCTG & \multirow{2}{*}{109} & \multirow{2}{*}{ ENSG00000197461 } \\
\hline & rev & GGGCCAGATCAGGAAGTTG & & \\
\hline \multirow{2}{*}{ human GAPDH } & fwd & $\begin{array}{l}\text { CCT CAA GAT CAT CAG CAA TGC } \\
\text { C }\end{array}$ & 189 & \multirow{2}{*}{ ENSG00000111640 } \\
\hline & rev & ATG TTC TGG AGA GCC CCG C & & \\
\hline \multirow{2}{*}{$t d R F P$} & fwd & AGAGTTCATGCGCTTCAAGGT & \multirow{2}{*}{121} & \\
\hline & rev & GCTTCTTGTAGT CGGGGATG & & \\
\hline
\end{tabular}




\section{A3. Antibodies and dyes}

\begin{tabular}{|c|c|c|c|c|c|c|}
\hline \multirow[b]{2}{*}{ Primary antibody } & \multirow[b]{2}{*}{ Species } & \multirow[b]{2}{*}{ Manufacturer } & \multirow[b]{2}{*}{ Cat. \# } & \multicolumn{3}{|c|}{ Dilution amount } \\
\hline & & & & Flow cytometry & Whole mount & WB \\
\hline Anti-CD117-PE & rat & Miltenyi Biotec & $130-091-730$ & $1: 33$ & - & - \\
\hline Anti-CD140a (PDGFRa)-PE & rat & eBioscience & $12-1401$ & $1: 500$ & - & - \\
\hline Anti-CD105-APC & rat & Miltenyi Biotec & $130-092-930$ & $1: 11$ & - & - \\
\hline Anti-Sca-1-PE & rat & Miltenyi Biotec & $130-102-832$ & $1: 33$ & - & - \\
\hline Anti-CD117-BV421 & mouse & BD Horizon $^{\mathrm{TM}}$ & 562434 & $1: 20$ & - & - \\
\hline Anti-CD31-FITC & mouse & BD Pharmingen & 557508 & $1: 20$ & - & - \\
\hline Anti-CD31-BV510 & mouse & BD Horizon $^{\mathrm{TM}}$ & 563454 & $1: 20$ & - & - \\
\hline Anti-CD90-BUV395, & mouse & BD Horizon ${ }^{\mathrm{TM}}$ & 563804 & $1: 20$ & - & - \\
\hline Anti-CD105-PerCp-Cy 5.5 & mouse & BD Horizon $^{\mathrm{TM}}$ & 560819 & $1: 20$ & - & - \\
\hline Anti-CD45-FITC & mouse & BD Pharmingen & 555482 & $1: 20$ & - & - \\
\hline Anti- $\alpha$-Actinin (Sarcomeric) & mouse & Sigma-Aldrich & A7811 & $1: 4000$ & $1: 1000$ & - \\
\hline Anti-Ki67 & rabbit & Thermoscientific & RM-9106-S0 & $1: 50$ & $1: 200$ & - \\
\hline Anti-active caspase- 3 & rabbit & Promega & G748 & - & $1: 250$ & - \\
\hline Anti-GFP & rabbit & Abcam & ab290 & - & $1: 500$ & - \\
\hline Anti-HIF-1a & mouse & BD Transduction & 610959 & - & - & $1: 1000$ \\
\hline Anti- $\beta$-actin & mouse & Sigma-Aldrich & A2228 & - & - & $1: 5000$ \\
\hline
\end{tabular}

\begin{tabular}{|l|l|l|l|c|c|c|}
\cline { 5 - 7 } & \multicolumn{3}{c|}{ Dilution amount } \\
\hline Secondary antibody & Species & Manufacturer & Cat. \# & Flow cytometry & Whole mount & WB \\
\hline anti-mouse-Alexa Fluor 488 & goat & Invitrogen & A11001 & $1: 1000$ & $1: 1000$ & - \\
\hline anti-mouse-Alexa Fluor 546 & goat & Invitrogen & A11003 & $1: 1000$ & $1: 1000$ & - \\
\hline anti-mouse-Alexa Fluor 633 & goat & Invitrogen & A-21050 & $1: 1000$ & $1: 1000$ & - \\
\hline Anti-mouse-IgG-HRP conjugated & mouse & Dako & P0260 & - & - & $1: 10000$ \\
\hline
\end{tabular}

\begin{tabular}{|l|l|l|c|c|}
\cline { 3 - 4 } \multicolumn{2}{c}{} & \multicolumn{2}{c|}{ Dilution amount } \\
\hline Dye & Manufacturer & Cat. \# & Flow cytometry & Whole mount \\
\hline Hoechst, $1 \mathrm{mg} / \mathrm{ml}$ & BD Biosciences & 33342 & $1: 1000$ & $1: 1000$ \\
\hline Sytox Red Dead Cell Stain-633 & Molecular Probes & S34859 & $1: 1000$ & $1: 1000$ \\
\hline
\end{tabular}




\section{A4. Life organisms}

\section{Animals:}

\begin{tabular}{|l|l|l|}
\hline Species & Background & Source \\
\hline Mus musculus & NMRI (CD1) & Animal facility, UMG \\
\hline Musmusculus & $\begin{array}{l}\text { FVB.129S6-Gt(ROSA26)Sor } \text { tm2(HIF1A/Luc) Kael/J: HIF-1a-ODD-Luc }_{\text {knock-in in ROSA26 locus, expression enhanced by fusion to }}^{\text {a CMV promoter element }} \\
\text { Rattus norvegicus }\end{array}$ Wistar & The Jackson laboratory \\
\hline
\end{tabular}

Cell lines:

\begin{tabular}{|c|c|c|}
\hline Cell line & Genetic background & Source \\
\hline mESCs- $\alpha M H C-N e o R$ & $\begin{array}{l}\text { mESC-R1 line (Nagy et al. 1993) with random } \\
\text { integration of neoR gene under transcriptional control } \\
\text { by cardiomyocyte specific aMHC promoter element }\end{array}$ & $\begin{array}{l}\text { Institute of Pharmacology and } \\
\text { Toxicology }\end{array}$ \\
\hline $\begin{array}{l}\text { mESCs-HIF-1a-ODD- } \\
\text { Luc/aMHC-neoR }\end{array}$ & $\begin{array}{l}\text { Transgenic ROSA26 ODD-Luc/+ mice derived mESCs } \\
\text { ubiquitiously expressing HIF-1a-ODD-Luc fusion } \\
\text { protein and neomycin resistance (neoR) under } \\
\text { transciptional control by aMHC promoter element) }\end{array}$ & $\begin{array}{l}\text { Isolation and transgenic } \\
\text { modification of the stem cell line } \\
\text { by Andreas Schraut (Institute of } \\
\text { Pharmacology and Toxicology) }\end{array}$ \\
\hline $\mathrm{mCPCs}\left(\mathrm{GFP}^{+}\right)$ & $\begin{array}{l}\text { Mouse cardiac progenitor cells isolated from adult } \\
\text { mouse heart with retrovirus transduction of EGFP }\end{array}$ & $\begin{array}{l}\text { Kindly provided by Mark } \\
\text { Sussman (Fischer et al. 2009) }\end{array}$ \\
\hline hCPCs $\left(\mathrm{GFP}^{+}\right)$ & $\begin{array}{l}\text { Human cardiac progenitor cells isolated from atrial } \\
\text { appendage of adult human heart with lentiviral } \\
\text { transduction of EGFP }\end{array}$ & $\begin{array}{l}\text { Kindly provided by Coretherapix } \\
\text { Laboratory (Lauden et al. 2013) }\end{array}$ \\
\hline hFFs $\left(\right.$ GFP $\left.^{+}\right)$ & $\begin{array}{l}\text { Human foreskin fibroblasts with lentiviral transduction } \\
\text { of EGFP }\end{array}$ & $\begin{array}{l}\text { wild type hFFs; ATCC, \#SCRC- } \\
1041\end{array}$ \\
\hline hES2 RFP & $\begin{array}{l}\text { HES2 line (Embryonic Stem cell International) } \\
\text { (Reubinoff et al. 2000) with tdRFP knock-in in ROSA26 } \\
\text { locus }\end{array}$ & $\begin{array}{l}\text { Kind gift by Gordon Keller (Irion } \\
\text { et al. 2007) }\end{array}$ \\
\hline $\begin{array}{l}\text { hES2-HIF-1 } \alpha-O D D- \\
\text { Luc }\end{array}$ & $\begin{array}{l}\text { HES2 line with random integration of HIF-1a-ODD-Luc } \\
\text { fusion protein under Cytomegalovirus (CMV) promoter } \\
\text { element }\end{array}$ & $\begin{array}{l}\text { Trangenic modification of the } \\
\text { stem cell line by Dr. Claudia } \\
\text { Noack and Krasimira Sharkova }\end{array}$ \\
\hline Other cell types & Genetic background & Source \\
\hline mEFs & NMRI mice & See section 2.2 .3 \\
\hline $\mathrm{mCM}$ & $\begin{array}{l}\text { Mouse cardiomyocytes from mESC-HIF-1a-ODD- } \\
\text { Luc/aMHC-neoR }\end{array}$ & See section 2.1 .2 \\
\hline hCM & Human cardiomyocytes from hES2 RFP & See section 2.1 .3 \\
\hline ODD-Luc hCM & $\begin{array}{l}\text { Human cardiomyocytes from hES2 RFP-HIF-1a-ODD- } \\
\text { Luc }\end{array}$ & See section 2.7 .5 \\
\hline
\end{tabular}




\section{A5. Human CPC specific differentially expressed genes}

Table 17. Differentially expressed plasma membrane genes in hCPCs compared to hCFs and hFFs.

\begin{tabular}{|c|c|}
\hline Gene symbol & Gene Name \\
\hline HTR1D & 5-hydroxytryptamine (serotonin) receptor 1D \\
\hline HTR1F & 5-hydroxytryptamine (serotonin) receptor $1 \mathrm{~F}$ \\
\hline HTR7 & 5-hydroxytryptamine (serotonin) receptor 7 (adenylate cyclase-coupled) \\
\hline $\begin{array}{l}\text { PALM2- } \\
\text { AKAP2, } \\
\text { AKAP2 }\end{array}$ & A kinase (PRKA) anchor protein 2; paralemmin 2; PALM2-AKAP2 readthrough transcript \\
\hline ADAM29 & ADAM metallopeptidase domain 29 \\
\hline ART1 & ADP-ribosyltransferase 1 \\
\hline ABCC9 & ATP-binding cassette, sub-family C (CFTR/MRP), member 9 \\
\hline ABCD2 & ATP-binding cassette, sub-family D (ALD), member 2 \\
\hline ATP2B2 & ATPase, Ca++ transporting, plasma membrane 2 \\
\hline ATP6V1B1 & ATPase, $\mathrm{H}+$ transporting, lysosomal $56 / 58 \mathrm{kDa}, \mathrm{V} 1$ subunit $\mathrm{B} 1$ \\
\hline ATP4A & ATPase, $\mathrm{H}+/ \mathrm{K}+$ exchanging, alpha polypeptide \\
\hline CD22 & CD22 molecule \\
\hline CASS4 & Cas scaffolding protein family member 4 \\
\hline DSCAM & Down syndrome cell adhesion molecule \\
\hline EGFLAM & EGF-like, fibronectin type III and laminin G domains \\
\hline EHD1 & EH-domain containing 1 \\
\hline EHD2 & EH-domain containing 2 \\
\hline EPHB6 & EPH receptor B6 \\
\hline FCER1G & Fc fragment of $\lg E$, high affinity I, receptor for; gamma polypeptide \\
\hline GPR34 & G protein-coupled receptor 34 \\
\hline GPR37 & G protein-coupled receptor 37 (endothelin receptor type B-like) \\
\hline GPR39 & G protein-coupled receptor 39 \\
\hline GPR4 & G protein-coupled receptor 4 \\
\hline GPR65 & G protein-coupled receptor 65 \\
\hline INADL & InaD-like (Drosophila) \\
\hline LIMS1 & LIM and senescent cell antigen-like domains 1 \\
\hline MICB & MHC class I polypeptide-related sequence B \\
\hline NOX4 & NADPH oxidase 4 \\
\hline NCKAP1L & NCK-associated protein 1-like \\
\hline $\mathrm{NOTCH} 4$ & Notch homolog 4 (Drosophila) \\
\hline RAB13 & RAB13, member RAS oncogene family; similar to hCG24991 \\
\hline RAB17 & RAB17, member RAS oncogene family \\
\hline RAB3A & RAB3A, member RAS oncogene family \\
\hline RAB43 & RAB43, member RAS oncogene family; hypothetical LOC100131426 \\
\hline RHD & Rh blood group, $\mathrm{D}$ antigen \\
\hline ARHGDIA & Rho GDP dissociation inhibitor (GDI) alpha \\
\hline ARHGAP26 & Rho GTPase activating protein 26 \\
\hline
\end{tabular}


Appendix (A5. Human CPC specific differentially expressed genes)

\begin{tabular}{|c|c|}
\hline ARHGEF2 & Rho/Rac guanine nucleotide exchange factor (GEF) 2 \\
\hline SHKBP1 & SH3KBP1 binding protein 1 \\
\hline TCIRG1 & T-cell, immune regulator 1, ATPase, $\mathrm{H}+$ transporting, lysosomal V0 subunit A3 \\
\hline B4GALT1 & UDP-Gal:betaGlcNAc beta 1,4- galactosyltransferase, polypeptide 1 \\
\hline VSIG2 & V-set and immunoglobulin domain containing 2 \\
\hline ADI1 & acireductone dioxygenase 1 \\
\hline AFAP1 & actin filament associated protein 1 \\
\hline ACVRL1 & activin A receptor type II-like 1 \\
\hline ARC & activity-regulated cytoskeleton-associated protein \\
\hline ADCY3 & adenylate cyclase 3 \\
\hline ALK & anaplastic lymphoma receptor tyrosine kinase \\
\hline ANK1 & ankyrin 1 , erythrocytic \\
\hline ARSA & arylsulfatase $A$ \\
\hline ASGR1 & asialoglycoprotein receptor 1 \\
\hline ROS1 & c-ros oncogene 1 , receptor tyrosine kinase \\
\hline $\mathrm{CDH} 4$ & cadherin 4, type 1, R-cadherin (retinal) \\
\hline $\mathrm{CDH} 24$ & cadherin-like 24 \\
\hline CABP1 & calcium binding protein 1 \\
\hline CACNA1D & calcium channel, voltage-dependent, L type, alpha 1D subunit \\
\hline CACNA1B & calcium channel, voltage-dependent, $\mathrm{N}$ type, alpha 1B subunit \\
\hline CA2 & carbonic anhydrase II \\
\hline CADM1 & cell adhesion molecule 1 \\
\hline CCR10 & chemokine (C-C motif) receptor 10 \\
\hline CCR7 & chemokine (C-C motif) receptor 7 \\
\hline CHRM4 & cholinergic receptor, muscarinic 4 \\
\hline CHRNA1 & cholinergic receptor, nicotinic, alpha 1 (muscle) \\
\hline CHRNA9 & cholinergic receptor, nicotinic, alpha 9 \\
\hline CSPG5 & chondroitin sulfate proteoglycan 5 (neuroglycan C) \\
\hline CLTCL1 & clathrin, heavy chain-like 1 \\
\hline CLDN2 & claudin 2 \\
\hline CLDN24 & claudin-24-like \\
\hline F2RL3 & coagulation factor II (thrombin) receptor-like 3 \\
\hline COL13A1 & collagen, type XIII, alpha 1 \\
\hline COL23A1 & collagen, type XXIII, alpha 1 \\
\hline COL25A1 & collagen, type XXV, alpha 1 \\
\hline CPLX4 & complexin 4 \\
\hline CNTNAP1 & contactin associated protein 1 \\
\hline CTTNBP2 & cortactin binding protein 2 \\
\hline DDN & dendrin \\
\hline DRD4 & dopamine receptor D4 \\
\hline EDA & ectodysplasin A \\
\hline ESAM & endothelial cell adhesion molecule \\
\hline EFNB1 & ephrin-B1 \\
\hline EPB41L3 & erythrocyte membrane protein band 4.1 -like 3 \\
\hline ESR1 & estrogen receptor 1 \\
\hline
\end{tabular}




\begin{tabular}{|c|c|}
\hline FGA & fibrinogen alpha chain \\
\hline FLT4 & fms-related tyrosine kinase 4 \\
\hline FUT1 & fucosyltransferase 1 (galactoside 2-alpha-L-fucosyltransferase, $\mathrm{H}$ blood group) \\
\hline GABBR1 & gamma-aminobutyric acid (GABA) B receptor, 1 \\
\hline GABRR2 & gamma-aminobutyric acid (GABA) receptor, rho 2 \\
\hline GJB2 & gap junction protein, beta $2,26 \mathrm{kDa}$ \\
\hline GJC2 & gap junction protein, gamma $2,47 \mathrm{kDa}$ \\
\hline GRIN2C & glutamate receptor, ionotropic, $\mathrm{N}$-methyl $\mathrm{D}$-aspartate $2 \mathrm{C}$ \\
\hline GRIK2 & glutamate receptor, ionotropic, kainate 2 \\
\hline GRM2 & glutamate receptor, metabotropic 2 \\
\hline GRM6 & glutamate receptor, metabotropic 6 \\
\hline ENPEP & glutamyl aminopeptidase (aminopeptidase A) \\
\hline GLRA3 & glycine receptor, alpha 3 \\
\hline GHR & growth hormone receptor \\
\hline GNG2 & guanine nucleotide binding protein (G protein), gamma 2 \\
\hline GNG4 & guanine nucleotide binding protein (G protein), gamma 4 \\
\hline GNGT2 & guanine nucleotide binding protein (G protein), gamma transducing activity polypeptide 2 \\
\hline GBP2 & guanylate binding protein 2 , interferon-inducible \\
\hline HBEGF & heparin-binding EGF-like growth factor \\
\hline $\mathrm{HRH} 2$ & histamine receptor $\mathrm{H} 2$ \\
\hline HAS1 & hyaluronan synthase 1 \\
\hline IGSF9 & immunoglobulin superfamily, member 9 \\
\hline INPP5E & inositol polyphosphate-5-phosphatase, $72 \mathrm{kDa}$ \\
\hline IGF1R & insulin-like growth factor 1 receptor \\
\hline ITGA2B & integrin, alpha $2 \mathrm{~b}$ (platelet glycoprotein Ilb of Ilb/Illa complex, antigen CD41) \\
\hline ITGA5 & integrin, alpha 5 (fibronectin receptor, alpha polypeptide) \\
\hline ITGA8 & integrin, alpha 8 \\
\hline ITGAX & integrin, alpha $\mathrm{X}$ (complement component 3 receptor 4 subunit) \\
\hline ITGB2 & integrin, beta 2 (complement component 3 receptor 3 and 4 subunit) \\
\hline IL12RB2 & interleukin 12 receptor, beta 2 \\
\hline IL15 & interleukin 15 \\
\hline IL6 & interleukin 6 (interferon, beta 2) \\
\hline KLHL17 & kelch-like 17 (Drosophila) \\
\hline LZTS1 & leucine zipper, putative tumor suppressor 1 \\
\hline LILRB3 & leukocyte immunoglobulin-like receptor, subfamily B (with TM and ITIM domains), member 3 \\
\hline LTK & leukocyte receptor tyrosine kinase \\
\hline LIN7A & lin-7 homolog A (C. elegans) \\
\hline LRP1 & low density lipoprotein-related protein 1 (alpha-2-macroglobulin receptor) \\
\hline LRP2 & low density lipoprotein-related protein 2 \\
\hline LHCGR & luteinizing hormone/choriogonadotropin receptor \\
\hline LAG3 & lymphocyte-activation gene 3 \\
\hline MSR1 & macrophage scavenger receptor 1 \\
\hline HLA-DOA & major histocompatibility complex, class II, DO alpha \\
\hline MMP14 & matrix metallopeptidase 14 (membrane-inserted) \\
\hline MMP25 & matrix metallopeptidase 25 \\
\hline
\end{tabular}




\begin{tabular}{|c|c|}
\hline MTNR1A & melatonin receptor $1 \mathrm{~A}$ \\
\hline MPP2 & membrane protein, palmitoylated 2 (MAGUK p55 subfamily member 2) \\
\hline MPP7 & membrane protein, palmitoylated 7 (MAGUK p55 subfamily member 7) \\
\hline MAPK8IP3 & mitogen-activated protein kinase 8 interacting protein 3 \\
\hline MUC1 & mucin 1 , cell surface associated \\
\hline MYO7A & myosin VIIA \\
\hline MYH6 & myosin, heavy chain 6 , cardiac muscle, alpha \\
\hline MYH9 & myosin, heavy chain 9 , non-muscle \\
\hline NRG1 & neuregulin 1 \\
\hline NRXN1 & neurexin 1 \\
\hline NCF2 & neutrophil cytosolic factor 2 \\
\hline NEXN & nexilin ( $\mathrm{F}$ actin binding protein) \\
\hline OSM & oncostatin M \\
\hline OPCML & opioid binding protein/cell adhesion molecule-like \\
\hline PARD6B & par-6 partitioning defective 6 homolog beta (C. elegans) \\
\hline PARVG & parvin, gamma \\
\hline $\mathrm{PTCH} 2$ & patched homolog 2 (Drosophila) \\
\hline PHEX & phosphate regulating endopeptidase homolog, X-linked \\
\hline PDE4A & phosphodiesterase 4A, cAMP-specific (phosphodiesterase E2 dunce homolog, Drosophila) \\
\hline PLA2G4F & phospholipase A2, group IVF \\
\hline PHKA1 & phosphorylase kinase, alpha 1 pseudogene 1; phosphorylase kinase, alpha 1 (muscle) \\
\hline PODXL & podocalyxin-like \\
\hline PVRL2 & poliovirus receptor-related 2 (herpesvirus entry mediator B) \\
\hline PKD1 & polycystic kidney disease 1 (autosomal dominant) \\
\hline KCNN3 & potassium intermediate/small conductance calcium-activated channel, subfamily $\mathrm{N}$, member 3 \\
\hline KCNN4 & potassium intermediate/small conductance calcium-activated channel, subfamily N, member 4 \\
\hline KCNJ1 & potassium inwardly-rectifying channel, subfamily J, member 1 \\
\hline KCNJ14 & potassium inwardly-rectifying channel, subfamily J, member 14 \\
\hline KCNJ2 & potassium inwardly-rectifying channel, subfamily J, member 2 \\
\hline KCNMB4 & potassium large conductance calcium-activated channel, subfamily M, beta member 4 \\
\hline KCNS2 & potassium voltage-gated channel, delayed-rectifier, subfamily S, member 2 \\
\hline KCNG1 & potassium voltage-gated channel, subfamily G, member 1 \\
\hline PRR7 & proline rich 7 (synaptic) \\
\hline PTGER1 & prostaglandin E receptor 1 (subtype EP1), 42kDa \\
\hline PTPN3 & protein tyrosine phosphatase, non-receptor type 3 \\
\hline PTPRF & protein tyrosine phosphatase, receptor type, $\mathrm{F}$ \\
\hline PTPRH & protein tyrosine phosphatase, receptor type, $\mathrm{H}$ \\
\hline PCDH11X & protocadherin $11 \mathrm{X}$-linked \\
\hline PCDH12 & protocadherin 12 \\
\hline PCDHB15 & protocadherin beta 15 \\
\hline P2RY1 & purinergic receptor P2Y, G-protein coupled, 1 \\
\hline $\mathrm{RHOF}$ & ras homolog gene family, member F (in filopodia) \\
\hline RHOG & ras homolog gene family, member G (rho G) \\
\hline RAMP1 & receptor (G protein-coupled) activity modifying protein 1 \\
\hline RAMP2 & receptor (G protein-coupled) activity modifying protein 2 \\
\hline
\end{tabular}




\begin{tabular}{|c|c|}
\hline ROR2 & receptor tyrosine kinase-like orphan receptor 2 \\
\hline RAPSN & receptor-associated protein of the synapse \\
\hline RIMS3 & regulating synaptic membrane exocytosis 3 \\
\hline RXFP3 & relaxin/insulin-like family peptide receptor 3 \\
\hline ROM1 & retinal outer segment membrane protein 1 \\
\hline SGCA & sarcoglycan, alpha (50kDa dystrophin-associated glycoprotein) \\
\hline SELP & selectin $\mathrm{P}$ (granule membrane protein 140kDa, antigen CD62) \\
\hline SIRPB1 & signal-regulatory protein beta 1 \\
\hline SIT1 & signaling threshold regulating transmembrane adaptor 1 \\
\hline MLLT4 & $\begin{array}{l}\text { similar to Afadin (Protein AF-6); myeloid/lymphoid or mixed-lineage leukemia (trithorax homolog, } \\
\text { Drosophila); translocated to, } 4\end{array}$ \\
\hline SLC1A5 & solute carrier family 1 (neutral amino acid transporter), member 5 \\
\hline SLC12A7 & solute carrier family 12 (potassium/chloride transporters), member 7 \\
\hline SLC12A1 & solute carrier family 12 (sodium/potassium/chloride transporters), member 1 \\
\hline SLC13A5 & solute carrier family 13 (sodium-dependent citrate transporter), member 5 \\
\hline SLC16A3 & solute carrier family 16, member 3 (monocarboxylic acid transporter 4 ) \\
\hline SLC16A7 & solute carrier family 16, member 7 (monocarboxylic acid transporter 2) \\
\hline SLC2A1 & solute carrier family 2 (facilitated glucose transporter), member 1 \\
\hline SLC2A9 & solute carrier family 2 (facilitated glucose transporter), member 9 \\
\hline SLC22A13 & solute carrier family 22 (organic anion transporter), member 13 \\
\hline SLC22A5 & solute carrier family 22 (organic cation/carnitine transporter), member 5 \\
\hline SLC25A4 & solute carrier family 25 (mitochondrial carrier; adenine nucleotide translocator), member 4 \\
\hline SLC26A9 & solute carrier family 26 , member 9 \\
\hline SLC28A1 & solute carrier family 28 (sodium-coupled nucleoside transporter), member 1 \\
\hline SLC6A13 & solute carrier family 6 (neurotransmitter transporter, GABA), member 13 \\
\hline SLC7A1 & solute carrier family 7 (cationic amino acid transporter, y+ system), member 1 \\
\hline SLC7A9 & solute carrier family 7 (cationic amino acid transporter, y+ system), member 9 \\
\hline SLC8A1 & solute carrier family 8 (sodium/calcium exchanger), member 1 \\
\hline SLC9A1 & solute carrier family 9 (sodium/hydrogen exchanger), member 1 \\
\hline SLC9A3R2 & solute carrier family 9 (sodium/hydrogen exchanger), member 3 regulator 2 \\
\hline SORBS3 & sorbin and $\mathrm{SH} 3$ domain containing 3 \\
\hline S1PR3 & sphingosine-1-phosphate receptor 3 \\
\hline STOM & stomatin \\
\hline STX3 & syntaxin 3 \\
\hline TLN1 & talin 1 \\
\hline TNS4 & tensin 4 \\
\hline TBXA2R & thromboxane A2 receptor \\
\hline TPO & thyroid peroxidase \\
\hline TRHR & thyrotropin-releasing hormone receptor \\
\hline TFRC & transferrin receptor $(p 90, C D 71)$ \\
\hline TGFB1I1 & transforming growth factor beta 1 induced transcript 1 \\
\hline TGFBR2 & transforming growth factor, beta receptor II $(70 / 80 \mathrm{kDa})$ \\
\hline TGM3 & transglutaminase 3 (E polypeptide, protein-glutamine-gamma-glutamyltransferase) \\
\hline TRPC5 & transient receptor potential cation channel, subfamily C, member 5 \\
\hline TRPM2 & transient receptor potential cation channel, subfamily M, member 2 \\
\hline
\end{tabular}


Appendix (A5. Human CPC specific differentially expressed genes)

\begin{tabular}{|l|l|} 
TM4SF1 & transmembrane $4 \mathrm{~L}$ six family member 1 \\
\hline TMPRSS9 & transmembrane protease, serine 9 \\
\hline TMEM204 & transmembrane protein 204 \\
\hline TNF & tumor necrosis factor (TNF superfamily, member 2) \\
\hline TNFSF11 & tumor necrosis factor (ligand) superfamily, member 11 \\
\hline TNFSF8 & tumor necrosis factor (ligand) superfamily, member 8 \\
\hline VASP & vasodilator-stimulated phosphoprotein \\
\hline VEPH1 & ventricular zone expressed PH domain homolog 1 (zebrafish) \\
\hline VAMP1 & vesicle-associated membrane protein 1 (synaptobrevin 1) \\
\hline XIRP1 & xin actin-binding repeat containing 1 \\
\hline ZAP70 & zeta-chain (TCR) associated protein kinase 70kDa \\
\hline ZYX & zyxin \\
\hline
\end{tabular}

Table 18. Differentially expressed cell adhesion genes in hCPCs compared to hCFs and hFFs.

\begin{tabular}{|c|c|}
\hline Gene symbol & Gene Name \\
\hline ADAM22 & ADAM metallopeptidase domain 22 \\
\hline AEBP1 & AE binding protein 1 \\
\hline CD22 & CD22 molecule \\
\hline CUZD1 & CUB and zona pellucida-like domains 1 \\
\hline CASS4 & Cas scaffolding protein family member 4 \\
\hline CDON & Cdon homolog (mouse) \\
\hline DSCAM & Down syndrome cell adhesion molecule \\
\hline LIMS1 & LIM and senescent cell antigen-like domains 1 \\
\hline RAB13 & RAB13, member RAS oncogene family; similar to hCG24991 \\
\hline RADIL & Ras association and DIL domains \\
\hline B4GALT1 & UDP-Gal:betaGlcNAc beta 1,4- galactosyltransferase, polypeptide 1 \\
\hline WISP2 & WNT1 inducible signaling pathway protein 2 \\
\hline AOC3 & amine oxidase, copper containing 3 (vascular adhesion protein 1) \\
\hline BMP1 & bone morphogenetic protein 1 \\
\hline $\mathrm{CDH} 16$ & cadherin 16 , KSP-cadherin \\
\hline $\mathrm{CDH} 4$ & cadherin 4, type 1, R-cadherin (retinal) \\
\hline $\mathrm{CDH} 23$ & cadherin-like 23 \\
\hline $\mathrm{CDH} 24$ & cadherin-like 24 \\
\hline CLSTN2 & calsyntenin 2 \\
\hline CPXM1 & carboxypeptidase $X$ (M14 family), member 1 \\
\hline CTNNAL1 & catenin (cadherin-associated protein), alpha-like 1 \\
\hline CADM1 & cell adhesion molecule 1 \\
\hline CADM4 & cell adhesion molecule 4 \\
\hline CLDN2 & claudin 2 \\
\hline COL6A3 & collagen, type VI, alpha 3 \\
\hline COL13A1 & collagen, type XIII, alpha 1 \\
\hline COL20A1 & collagen, type $\mathrm{XX}$, alpha 1 \\
\hline COL27A1 & collagen, type XXVII, alpha 1 \\
\hline
\end{tabular}


Appendix (A5. Human CPC specific differentially expressed genes)

\begin{tabular}{|c|c|}
\hline CNTN3 & contactin 3 (plasmacytoma associated) \\
\hline CNTNAP1 & contactin associated protein 1 \\
\hline CNTNAP2 & contactin associated protein-like 2 \\
\hline CYR61 & cysteine-rich, angiogenic inducer, 61 \\
\hline DPT & dermatopontin \\
\hline DAB1 & disabled homolog 1 (Drosophila) \\
\hline EDA & ectodysplasin A \\
\hline EMILIN1 & elastin microfibril interfacer 1 \\
\hline ESAM & endothelial cell adhesion molecule \\
\hline EFNB1 & ephrin-B1 \\
\hline FBLN7 & fibulin 7 \\
\hline FPR2 & formyl peptide receptor 2 \\
\hline HES1 & hairy and enhancer of split 1, (Drosophila) \\
\hline HAS1 & hyaluronan synthase 1 \\
\hline ITGA2B & integrin, alpha 2b (platelet glycoprotein Ilb of IIb/IIla complex, antigen CD41) \\
\hline ITGA5 & integrin, alpha 5 (fibronectin receptor, alpha polypeptide) \\
\hline ITGA8 & integrin, alpha 8 \\
\hline ITGAX & integrin, alpha $X$ (complement component 3 receptor 4 subunit) \\
\hline ITGB2 & integrin, beta 2 (complement component 3 receptor 3 and 4 subunit) \\
\hline IL32 & interleukin 32 \\
\hline LAMA1 & laminin, alpha 1 \\
\hline LAMA2 & laminin, alpha 2 \\
\hline LSAMP & limbic system-associated membrane protein \\
\hline MCAM & melanoma cell adhesion molecule \\
\hline MTSS1 & metastasis suppressor 1 \\
\hline MAG & myelin associated glycoprotein \\
\hline MYH9 & myosin, heavy chain 9 , non-muscle \\
\hline NPHP4 & nephronophthisis 4 \\
\hline NRXN1 & neurexin 1 \\
\hline NFASC & neurofascin homolog (chicken) \\
\hline NLGN2 & neuroligin 2 \\
\hline NLGN4Y & neuroligin 4, Y-linked \\
\hline NRP2 & neuropilin 2 \\
\hline NTM & neurotrimin \\
\hline OPCML & opioid binding protein/cell adhesion molecule-like \\
\hline PARVG & parvin, gamma \\
\hline PVRL2 & poliovirus receptor-related 2 (herpesvirus entry mediator B) \\
\hline PKD1 & polycystic kidney disease 1 (autosomal dominant) \\
\hline PSTPIP1 & proline-serine-threonine phosphatase interacting protein 1 \\
\hline PTPRF & protein tyrosine phosphatase, receptor type, F \\
\hline PCDH11X & protocadherin 11 X-linked \\
\hline PCDH12 & protocadherin 12 \\
\hline PCDHB15 & protocadherin beta 15 \\
\hline PCDHGA11 & protocadherin gamma subfamily A, 11 \\
\hline PCDHGA5 & protocadherin gamma subfamily A, 5 \\
\hline
\end{tabular}


Appendix (A5. Human CPC specific differentially expressed genes)

\begin{tabular}{|l|l|} 
PCDHGA6 & protocadherin gamma subfamily A, 6 \\
\hline PCDHGA7 & protocadherin gamma subfamily A, 7 \\
\hline PCDHGA8 & protocadherin gamma subfamily A, 8 \\
\hline PCDHGB2 & protocadherin gamma subfamily B, 2 \\
\hline PCDHGB3 & protocadherin gamma subfamily B, 3 \\
\hline PCDHGB4 & protocadherin gamma subfamily B, 4 \\
\hline PCDHGB6 & protocadherin gamma subfamily B, 6 \\
\hline PCDHGC5, & protocadherin gamma subfamily C, 5 \\
\hline PCDHGC3 & receptor tyrosine kinase-like orphan receptor 2 \\
\hline ROR2 & reelin \\
\hline RELN & retinal outer segment membrane protein 1 \\
\hline ROM1 & retinoschisin 1 \\
\hline RS1 & scavenger receptor class F, member 2 \\
\hline SCARF2 & selectin P (granule membrane protein 140kDa, antigen CD62) \\
\hline SELP & similar to Afadin (Protein AF-6); myeloid/lymphoid or mixed-lineage leukemia (trithorax \\
\hline MLLT4 & somolog, Drosophila); translocated to, 4 \\
\hline SORBS3 & sperm acrosome associated 4 \\
\hline SPACA4 & sushi, nidogen and EGF-like domains 1 \\
\hline SNED1 & thrombospondin 4 \\
\hline THBS4 & transforming growth factor beta 1 induced transcript 1 \\
\hline TGFB111 & tumor necrosis factor (TNF superfamily, member 2) \\
\hline TNF & zyxin \\
\hline ZYX & \\
\hline
\end{tabular}

Table 19. Differentially expressed genes involved in actin cytoskeleton organization in hCPCs compared to hCFs and hFFs.

\begin{tabular}{|l|l|}
\hline Gene symbol & Gene Name \\
\hline CDC42BPG & CDC42 binding protein kinase gamma (DMPK-like) \\
\hline CDC42EP2 & CDC42 effector protein (Rho GTPase binding) 2 \\
\hline EHD2 & EH-domain containing 2 \\
\hline NCK2 & NCK adaptor protein 2 \\
\hline NUAK2 & NUAK family, SNF1-like kinase, 2 \\
\hline PDLIM7 & PDZ and LIM domain 7 (enigma) \\
\hline ARHGAP26 & Rho GTPase activating protein 26 \\
\hline ARHGEF2 & Rho/Rac guanine nucleotide exchange factor (GEF) 2 \\
\hline WIPF1 & WAS/WASL interacting protein family, member 1 \\
\hline ACTC1 & actin, alpha, cardiac muscle 1 \\
\hline ACTN4 & actinin, alpha 4 \\
\hline ALDOA & aldolase A, fructose-bisphosphate \\
\hline CXCL1 & chemokine (C-X-C motif) ligand 1 (melanoma growth stimulating activity, alpha) \\
\hline DAAM2 & dishevelled associated activator of morphogenesis 2 \\
\hline DBN1 & drebrin 1 \\
\hline EPB41 & erythrocyte membrane protein band 4.1 (elliptocytosis 1, RH-linked) \\
\hline
\end{tabular}


Appendix (A5. Human CPC specific differentially expressed genes)

\begin{tabular}{|c|c|}
\hline EPB41L3 & erythrocyte membrane protein band 4.1-like 3 \\
\hline FSCN1 & fascin homolog 1, actin-bundling protein (Strongylocentrotus purpuratus) \\
\hline FLNA & filamin A, alpha (actin binding protein 280 ) \\
\hline FLNB & filamin B, beta (actin binding protein 278 ) \\
\hline FOXJ1 & forkhead box J1 \\
\hline FMNL3 & formin-like 3 \\
\hline GHRL & ghrelin/obestatin prepropeptide \\
\hline GHSR & growth hormone secretagogue receptor \\
\hline INF2 & inverted formin, $\mathrm{FH} 2$ and $\mathrm{WH} 2$ domain containing \\
\hline MTSS1 & metastasis suppressor 1 \\
\hline MYO9B & myosin IXB \\
\hline MYH6 & myosin, heavy chain 6 , cardiac muscle, alpha \\
\hline MYH9 & myosin, heavy chain 9 , non-muscle \\
\hline NPHP4 & nephronophthisis 4 \\
\hline PLEK2 & pleckstrin 2 \\
\hline RHOF & ras homolog gene family, member $\mathrm{F}$ (in filopodia) \\
\hline RAC2 & ras-related C3 botulinum toxin substrate 2 (rho family, small GTP binding protein Rac2) \\
\hline SPTBN5 & spectrin, beta, non-erythrocytic 5 \\
\hline TLN1 & talin 1 \\
\hline TMSB10 & thymosin beta 10 \\
\hline VASP & vasodilator-stimulated phosphoprotein \\
\hline XIRP1 & xin actin-binding repeat containing 1 \\
\hline
\end{tabular}

Table 20. Differentially expressed extracellular region genes in hCPCs compared to hCFs and hFFs.

\begin{tabular}{|l|l|}
\hline Gene symbol & Gene Name \\
\hline AGPAT1 & 1-acylglycerol-3-phosphate O-acyltransferase 1 (lysophosphatidic acid acyltransferase, alpha) \\
\hline ADAMTS3 & ADAM metallopeptidase with thrombospondin type 1 motif, 3 \\
\hline ADAMTSL4 & ADAMTS-like 4 \\
\hline ADAMTSL5 & ADAMTS-like 5 \\
\hline C1QTNF2 & C1q and tumor necrosis factor related protein 2 \\
\hline CD248 & CD248 molecule, endosialin \\
\hline CMTM4 & CKLF-like MARVEL transmembrane domain containing 4 \\
\hline CMTM7 & CKLF-like MARVEL transmembrane domain containing 7 \\
\hline TIMP1 & TIMP metallopeptidase inhibitor 1 \\
\hline ACHE & acetylcholinesterase (Yt blood group) \\
\hline AHSG & alpha-2-HS-glycoprotein \\
\hline ANGPTL2 & angiopoietin-like 2 \\
\hline ANGPTL3 & angiopoietin-like 3 \\
\hline APOB & apolipoprotein B (including Ag(x) antigen) \\
\hline APOC1 & apolipoprotein C-I \\
\hline BMP5 & bone morphogenetic protein 5 \\
\hline BCAN & brevican \\
\hline CDH13 & cadherin 13, H-cadherin (heart) \\
\hline
\end{tabular}


Appendix (A5. Human CPC specific differentially expressed genes)

\begin{tabular}{|c|c|}
\hline CPB2 & carboxypeptidase B2 (plasma) \\
\hline $\mathrm{CP}$ & ceruloplasmin (ferroxidase) \\
\hline CCL2 & chemokine (C-C motif) ligand 2 \\
\hline CCL7 & chemokine (C-C motif) ligand 7 \\
\hline CXCL5 & chemokine (C-X-C motif) ligand 5 \\
\hline COL6A6 & collagen type VI alpha 6 \\
\hline COL4A6 & collagen, type IV, alpha 6 \\
\hline COL9A2 & collagen, type IX, alpha 2 \\
\hline COL9A3 & collagen, type IX, alpha 3 \\
\hline COL7A1 & collagen, type VII, alpha 1 \\
\hline COL8A2 & collagen, type VIII, alpha 2 \\
\hline COL14A1 & collagen, type XIV, alpha 1 \\
\hline CSF2 & colony stimulating factor 2 (granulocyte-macrophage) \\
\hline CFD & complement factor D (adipsin) \\
\hline CFI & complement factor I \\
\hline CRISPLD2 & cysteine-rich secretory protein LCCL domain containing 2 \\
\hline CRLF1 & cytokine receptor-like factor 1 \\
\hline ELN & elastin \\
\hline EMILIN3 & elastin microfibril interfacer 3 \\
\hline EDN1 & endothelin 1 \\
\hline ECM1 & extracellular matrix protein 1 \\
\hline ECM2 & extracellular matrix protein 2 , female organ and adipocyte specific \\
\hline FBN2 & fibrillin 2 \\
\hline FGB & fibrinogen beta chain \\
\hline FJX1 & four jointed box 1 (Drosophila) \\
\hline GSN & gelsolin (amyloidosis, Finnish type) \\
\hline GPC1 & glypican 1 \\
\hline GRN & granulin \\
\hline GREM1 & gremlin 1, cysteine knot superfamily, homolog (Xenopus laevis) \\
\hline GDF7 & growth differentiation factor 7 \\
\hline HAPLN3 & hyaluronan and proteoglycan link protein 3 \\
\hline IGFBP1 & insulin-like growth factor binding protein 1 \\
\hline IGFBP5 & insulin-like growth factor binding protein 5 \\
\hline IBSP & integrin-binding sialoprotein \\
\hline ICAM1 & intercellular adhesion molecule 1 \\
\hline IL24 & interleukin 24 \\
\hline IL33 & interleukin 33 \\
\hline IL4 & interleukin 4 \\
\hline LAMA4 & laminin, alpha 4 \\
\hline LAMB3 & laminin, beta 3 \\
\hline LEFTY1 & left-right determination factor 1 \\
\hline LIF & leukemia inhibitory factor (cholinergic differentiation factor) \\
\hline LOX & lysyl oxidase \\
\hline LOXL4 & lysyl oxidase-like 4 \\
\hline MGP & matrix Gla protein \\
\hline
\end{tabular}


Appendix (A5. Human CPC specific differentially expressed genes)

\begin{tabular}{|c|c|}
\hline MMP16 & matrix metallopeptidase 16 (membrane-inserted) \\
\hline METRN & meteorin, glial cell differentiation regulator \\
\hline NPPB & natriuretic peptide precursor $B$ \\
\hline NENF & neuron derived neurotrophic factor \\
\hline OSTN & osteocrin \\
\hline OGN & osteoglycin \\
\hline PDGFC & platelet derived growth factor $\mathrm{C}$ \\
\hline PTN & pleiotrophin \\
\hline $\mathrm{PMCH}$ & pro-melanin-concentrating hormone \\
\hline PCSK5 & proprotein convertase subtilisin/kexin type 5 \\
\hline PCSK6 & proprotein convertase subtilisin/kexin type 6 \\
\hline PRSS36 & protease, serine, 36 \\
\hline SELE & selectin $\mathrm{E}$ \\
\hline SEMA3F & sema domain, immunoglobulin domain $(\mathrm{lg})$, short basic domain, secreted, (semaphorin) 3F \\
\hline SERPINA1 & serpin peptidase inhibitor, clade A (alpha-1 antiproteinase, antitrypsin), member 1 \\
\hline SPOCK3 & sparc/osteonectin, cwcv and kazal-like domains proteoglycan (testican) 3 \\
\hline SFTPB & surfactant protein B \\
\hline TNXB & tenascin XB; tenascin XA pseudogene \\
\hline THSD4 & thrombospondin, type I, domain containing 4 \\
\hline TGFB2 & transforming growth factor, beta 2 \\
\hline TNFAIP2 & tumor necrosis factor, alpha-induced protein 2 \\
\hline ERBB3 & v-erb-b2 erythroblastic leukemia viral oncogene homolog 3 (avian) \\
\hline VCAM1 & vascular cell adhesion molecule 1 \\
\hline VEGFC & vascular endothelial growth factor $\mathrm{C}$ \\
\hline VIT & vitrin \\
\hline VTN & vitronectin \\
\hline WNT16 & wingless-type MMTV integration site family, member 16 \\
\hline WNT5A & wingless-type MMTV integration site family, member $5 \mathrm{~A}$ \\
\hline WNT7A & wingless-type MMTV integration site family, member 7A \\
\hline WNT9A & wingless-type MMTV integration site family, member $9 \mathrm{~A}$ \\
\hline ZP3 & zona pellucida glycoprotein 3 (sperm receptor) \\
\hline
\end{tabular}



hFFs)

\section{A6. Growth factors and cytokines differentially expressed in hCPCs and hFFs}

Table 21. hCPC specific up-regulated growth factors and cytokines compared to hFFs.

\begin{tabular}{|c|c|}
\hline Gene symbol & Gene name \\
\hline FGF1 & Fibroblast Growth Factor 1 (Acidic) \\
\hline IL15 & Interleukin 15 \\
\hline PDGFA & Platelet-Derived Growth Factor Alpha Polypeptide \\
\hline TYMP & Thymidine Phosphorylase \\
\hline TNFSF9 & Tumor Necrosis Factor (Ligand) Superfamily, Member 9 \\
\hline TGFB3 & Transforming Growth Factor, Beta 3 \\
\hline ANGPT2 & Angiopoietin 2 \\
\hline CFP & Complement Factor Properdin \\
\hline IL17B & Interleukin 17B \\
\hline TNFSF10 & Tumor Necrosis Factor (Ligand) Superfamily, Member 10 \\
\hline IL13 & Interleukin 13 \\
\hline TNF & Tumor Necrosis Factor \\
\hline FGF8 & Fibroblast Growth Factor 8 (Androgen-Induced) \\
\hline IFNA8 & Interferon, Alpha 8 \\
\hline BMP8A & Bone Morphogenetic Protein 8a \\
\hline LHB & Luteinizing Hormone Beta Polypeptide \\
\hline FGF16 & Fibroblast Growth Factor 16 \\
\hline IL18BP & Interleukin 18 Binding Protein \\
\hline CFHR3 & Complement Factor H-Related 3 \\
\hline IL16 & Interleukin 16 \\
\hline IL18 & Interleukin 18 \\
\hline PDGFB & Platelet-Derived Growth Factor Beta Polypeptide \\
\hline TNFSF8 & Tumor Necrosis Factor (Ligand) Superfamily, Member 8 \\
\hline ERAP1 & Endoplasmic Reticulum Aminopeptidase 1 \\
\hline MDK & Midkine (Neurite Growth-Promoting Factor 2) \\
\hline IL32 & Interleukin 32 \\
\hline GDNF & Glial Cell Derived Neurotrophic Factor \\
\hline PGF & Placental Growth Factor \\
\hline HBEGF & Heparin-Binding EGF-Like Growth Factor \\
\hline BMP1 & Bone Morphogenetic Protein 1 \\
\hline CXCL3 & Chemokine (C-X-C Motif) Ligand 3 \\
\hline JAG1 & Jagged 1 \\
\hline TGFB1 & Transforming Growth Factor, Beta 1 \\
\hline IL1A & Interleukin 1, Alpha \\
\hline $\mathrm{CFH}$ & Complement Factor $\mathrm{H}$ \\
\hline IL1B & Interleukin 1, Beta \\
\hline CXCL12 & Chemokine (C-X-C Motif) Ligand 12 \\
\hline VEGFA & Vascular Endothelial Growth Factor A \\
\hline IL6 & Interleukin 6 \\
\hline CXCL8 & Chemokine (C-X-C Motif) Ligand 8 \\
\hline CXCL1 & $\begin{array}{l}\text { Chemokine (C-X-C Motif) Ligand } 1 \text { (Melanoma Growth Stimulating } \\
\text { Activity, Alpha) }\end{array}$ \\
\hline
\end{tabular}


Appendix (A6. Growth factors and cytokines differentially expressed in hCPCs and hFFs)

Table 22. hFF specific up-regulated growth factors and cytokines compared to hCPCs.

\begin{tabular}{|l|l|}
\hline Gene symbol & Gene name \\
\hline FGF13 & Fibroblast Growth Factor 13 \\
\hline IGF1 & Insulin-Like Growth Factor 1 (Somatomedin C) \\
\hline CCL4 & Chemokine (C-C Motif) Ligand 4 \\
\hline CCL3L3 & Chemokine (C-C Motif) Ligand 3-Like 3 \\
\hline IL19 & Interleukin 19 \\
\hline NRG2 & Neuregulin 2 \\
\hline FIGF & C-Fos Induced Growth Factor (Vascular Endothelial Growth Factor D) \\
\hline GRP & Gastrin-Releasing Peptide \\
\hline CRH & Corticotropin Releasing Hormone \\
\hline EGF & Epidermal Growth Factor \\
\hline CXCL11 & Chemokine (C-X-C Motif) Ligand 11 \\
\hline TGFA & Transforming Growth Factor, Alpha \\
\hline CYTL1 & Cytokine-Like 1 \\
\hline CCL28 & Chemokine (C-C Motif) Ligand 28 \\
\hline TNFSF18 & umor Necrosis Factor (Ligand) Superfamily, Member 18 \\
\hline CXCL14 & Chemokine (C-X-C Motif) Ligand 14 \\
\hline CCL3 & Chemokine (C-C Motif) Ligand 3 \\
\hline PDGFD & Platelet Derived Growth Factor D \\
\hline IL34 & Interleukin 34 \\
\hline CSF2 & Colony Stimulating Factor 2 (Granulocyte-Macrophage) \\
\hline CXCL10 & Chemokine (C-X-C Motif) Ligand 10 \\
\hline BMP2 & Bone Morphogenetic Protein 2 \\
\hline HGF & Hepatocyte Growth Factor (Hepapoietin A; Scatter Factor) \\
\hline SPP1 & Secreted Phosphoprotein 1 \\
\hline NTF3 & Neurotrophin 3 \\
\hline TM7SF2 & Transmembrane 7 Superfamily Member 2 \\
\hline KITLG & KIT Ligand \\
\hline EREG & Epiregulin \\
\hline BDNF & Brain-Derived Neurotrophic Factor \\
\hline ANGPT1 & Angiopoietin 1 \\
\hline VEGFC & Vascular Endothelial Growth Factor C \\
\hline CCL2 & Chemokine (C-C Motif) Ligand 2 \\
\hline GDF15 & Growth Differentiation Factor 15 \\
\hline CSF1 & Colony Stimulating Factor 1 (Macrophage) \\
\hline FGF2 & Fibroblast Growth Factor 2 (Basic) \\
\hline GRN & Granulin \\
\hline
\end{tabular}

